Sobre a teoria das transformações de superfícies de curvatura constante 


\title{
Sobre a teoria das transformações superfícies de curvatura constante
}

\author{
Gabriela Pereira Sander
}

Orientadora: Profa. Dra. Irene Ignazia Onnis

Dissertação apresentada ao Instituto de Ciências Matemáticas e de Computação - ICMC-USP, como parte dos requisitos para obtenção do título de Mestre em Matemática.

USP - São Carlos

Fevereiro/2009 
Aos meus pais, Mariza e Sander, que me deram força para continuar meu caminho nos estudos.

Ao André, por seu amor. 


\section{Agradecimentos}

Agradeço primeiramente a Deus, por iluminar meus caminhos nos momentos mais difíceis.

A meus pais, em especial minha mãe, pelo amor dedicado a mim e pela força e incentivo em meus estudos, não me deixando desistir nos pequenos obstáculos da vida.

Ao André, por seu amor e carinho, principalmente nos momentos de fraqueza, me dando força para continuar.

A minha irmã, Giovana, e aos meus familiares, em especial minha vó, "Dona Flor", que rezaram por mim durante este tempo que passei longe e que vibraram comigo a cada conquista minha.

A minha orientadora, Profa. Irene, que me guiou nos estudos e foi uma grande amiga nos momentos de confidências.

Aos meus amigos de Bauru (incluindo os que já saíram de lá) que me alegraram a cada final de semana "em casa" e entenderam que, mesmo longe, nós estávamos perto.

Aos meus amigos de São Carlos que foram minha família durante as semanas de estudos, desesperos, conquistas, tristezas, alegrias.

Aos professores e funcionários da USP de São Carlos, que se esforçam para tornar a nossa Universidade cada dia melhor.

A CAPES pelo auxílio financeiro, fundamental para o desenvolvimento de minha dissertação.

Enfim, a todos que (de alguma maneira) contribuíram para a minha formação acadêmica; eu agradeço de todo meu coração. 


\section{Resumo}

A teoria das transformações de superfícies de curvatura constante começou, no fim do século XIX, com o trabalho [3] de A.V. Bäcklund e, em seguida, recebeu importantes contribuições por parte de diversos geômetras, entre eles, L. Bianchi e C. Guichard (veja, por exemplo, [5, 6, 7, 17]).

Nessa dissertação apresentamos alguns dos mais importantes resultados desse tópico da geometria diferencial que estão relacionados às superfícies de curvatura média (ou gaussiana não nula) constante. Tais superfícies estão associadas a soluções de equações diferenciais parciais de segunda ordem e não lineares.

A interpretação analítica da teoria das transformações de superfícies de curvatura constante nos capacita obter soluções dessas equações diferenciais parciais a partir de uma outra dada, mediante integração de um sistema de equações diferenciais, chamado transformação de Bäcklund. Então, os teoremas de permutabilidade fornecem uma "fórmula de superposição" para a construção algébrica de novas soluções. 


\section{Abstract}

The theory on transformations of surfaces with constant curvature begins, in the late nineteen century, with the article [3] of A.V. Bäcklund and, after, received important contributions from various geometricians, among others, L. Bianchi and C. Guichard (see, for example, $[5,6,7,17]$ ).

In this dissertation we outline some of the most important results on the theory of surfaces of constant mean (or gaussian) curvature. Such surfaces are associated to the solutions of nonlinear partial differential equations of second order.

The analytic interpretation of the theory on transformations of constant curvature surfaces provides a method of obtaining, from a given solution of these partial differential equations, a new solution of the same equation, by integrating a system of differential equations, called Bäcklund transformation. Then, the permutability theorems give a "superposition formula" to construct, algebraically, new solutions. 


\section{Conteúdo}

Introdução 1

1 O método do triedro móvel 3

1.1 Triedro móvel e as formas de conexão . . . . . . . . . . . . . . . . . . 3

1.2 Equações de estrutura e Lema de E. Cartan . . . . . . . . . . . . . . . . . 5

1.3 Alguns resultados importantes utilizando o método do triedro móvel . . . . . . 6

1.3.1 A primeira e a segunda formas quadráticas . . . . . . . . . . . . . 7

1.3.2 As equações de Gauss e Codazzi-Mainardi . . . . . . . . . . . . . . . . 8

2 Equações diferenciais associadas a superfícies de curvatura constante 13

2.1 Superfícies de curvatura média constante . . . . . . . . . . . . . . . 13

2.2 Superfícies de curvatura gaussiana constante positiva . . . . . . . . . . . . . . 19

2.3 Superfícies de curvatura gaussiana constante negativa . . . . . . . . . . . . 23

3 Congruências de retas pseudo-esféricas $\quad 31$

3.1 Congruência de retas . . . . . . . . . . . . . . . . . . . . . 31

3.1 .1 Superfícies paralelas . . . . . . . . . . . . . . . . . 32

3.2 Congruências pseudo-esféricas . . . . . . . . . . . . . . . . . . 38

3.3 Transformação de Bäcklund para a equação de Sine-Gordon . . . . . . . . . . . . 44

3.3.1 O Teorema de Permutabilidade de Bianchi . . . . . . . . . . . . . . 48

3.4 Transformação de Bäcklund para a equação de Sine-Gordon elíptica . . . . . . . 55

4 Congruências de Guichard $\quad 61$

4.1 Congruência de retas normal . . . . . . . . . . . . . . . . . 62

4.2 Sobre as deformações das congruências de retas normais . . . . . . . . . . . 64

4.2 .1 Os Teoremas de Guichard . . . . . . . . . . . . . . . . . . . . . . 67

4.3 Recíproca dos Teoremas de Guichard . . . . . . . . . . . . . . . . . . 75

4.3.1 Tratamento do Problema 4.0.5 para o caso $\mathrm{H}=0 \ldots \ldots \ldots$. . . . . . 75

4.3.2 Tratamento do Problema 4.0.5 para o caso $K>0$. . . . . . . . . 79

4.3.3 Tratamento do Problema 4.0.5 para o caso $K<0 \quad \ldots \ldots \ldots$. . . . . . 84

4.3.4 Algumas propriedades das congruências de Guichard . . . . . . . . . . . . 87

5 Transformações de superfícies de curvatura constante $\quad 91$

5.1 Considerações preliminares e funções associadas a $T \ldots \ldots \ldots \ldots$. . . . . . . . . . . . .

5.2 Transformações de superfícies mínimas . . . . . . . . . . . . . . . 96

5.3 Transformações de superfícies de curvatura gaussiana constante não nula . . . . . 103 
5.4 Interpretação analítica . . . . . . . . . . . . . . . . . . . . . . . . 112

5.4 .1 Superfícies mínimas . . . . . . . . . . . . . . . . . . . . 112

5.4.2 Superfícies de curvatura gaussiana constante não nula . . . . . . . . . . . 115

5.4.3 Composição de transformadas de Bäcklund dadas por Bianchi . . . . . . . 120

5.5 Superfícies de curvatura média constante não nula . . . . . . . . . . . . . . 125

$\begin{array}{lr}\text { A Teoria local das superfícies } & 129\end{array}$

A.1 Superfície parametrizada regular . . . . . . . . . . . . . . . . . . . . . 129

A.2 Primeira e segunda formas quadráticas . . . . . . . . . . . . . . 130

A.3 Curvatura média e curvatura gaussiana . . . . . . . . . . . . . . . 132

A.3.1 Classificação dos pontos de uma superfície . . . . . . . . . . . . . . 135

A.4 Superfícies de Weingarten . . . . . . . . . . . . . . . . . 136

A.5 Linha de curvatura; curva assintótica; direções conjugadas . . . . . . . . . . . . . 138

B Formas diferenciais $\quad 143$

B.1 Formas diferenciais em $\mathbb{R}^{n} \ldots \ldots \ldots \ldots \ldots \ldots$. . . . . . . . . . . . . . . . . . . . . .

B.2 Teorema de Frobenius . . . . . . . . . . . . . . . . . . . . 146

$\begin{array}{lr}\text { Bibliografia } & 147\end{array}$ 


\section{Introdução}

O principal objetivo desse trabalho é expor alguns aspectos da teoria das transformações de superfícies de curvatura média (ou gaussiana não nula) constante.

As primeiras pesquisas sobre este tópico de geometria diferencial foram publicadas (em [3]) no fim do século XIX pelo matemático sueco A. V. Bäcklund, o qual se ocupou do estudo das congruências de retas pseudo-esféricas provando que as mesmas só existem entre superfícies pseudo-esféricas (isto é, de curvatura gaussiana constante negativa).

Da prova deste resultado, conhecido como Teorema de Bäcklund, surge uma importante equação chamada de "transformação de Bäcklund". Integrando tal transformação, Bäcklund provou que dada uma superfície pseudo-esférica $S$, existe uma família a dois parâmetros de superfícies de mesma curvatura gaussiana e relacionadas a $S$ através de uma congruência pseudoesférica (Teorema de Integrabilidade 3.2.4).

A interpretação analítica de tais resultados permite obter uma família a dois parâmetros de soluções da equação de Sine-Gordon

$$
\phi_{u u}-\phi_{v v}=\operatorname{sen} \phi \cos \phi
$$

a partir de uma conhecida. Para a prova deste fato, utiliza-se a existência de uma correspondência biunívoca entre as soluções (não nulas) da equação (1) e as superfícies pseudo-esféricas de $\mathbb{R}^{3}$, a menos de um movimento rígido (veja Proposição 2.3.3).

Neste contexto é relevante o Teorema de Permutabilidade do matemático italiano L. Bianchi, que fornece uma "fórmula de superposição" que nos capacita construir algebricamente novas soluções da equação de Sine-Gordon.

No Capítulo 2 foi demonstrado que também as superfícies de curvatura gaussiana constante positiva estão relacionadas às soluções de uma equação diferencial, a saber, a de Sine-Gordon elíptica:

$$
\Delta \phi=-\operatorname{senh} \phi \cosh \phi .
$$

A integração da transformação de Bäcklund para esta equação e o (análogo do) Teorema de Permutabilidade constituem ferramentas importantes para obter novas soluções de (2).

Os temas mencionados até o presente momento são apresentados nos Capítulos 2 e 3 , em detalhes.

O Capítulo 4 é dedicado ao estudo da classe especial de congruência de retas ditas normais e de dois importantes teoremas de C. Guichard. Tais teoremas, como o mesmo L. Bianchi ressalta em [6], foram apresentados em 1899 pelo autor no trabalho [17], porém sem as demonstrações, e estão relacionados à teoria das deformações de congruências de retas normais. A importância 
deles está no fato que permitem obter pares de superfícies $S$ e $S^{s}$ de mesma curvatura média (ou gaussiana não nula) constante a partir de uma superfície $S_{0}$ isométrica a uma certa superfície de revolução (veja Corolários 4.2.7 e 4.2.11). Além disso, as superfícies $S$ e $S^{s}$ são simétricas em relação a $S_{0}$ e ambas ligadas a $S_{0}$ por uma congruência de Guichard.

Com base nestes resultados, L. Bianchi trata em [6] do seguinte problema:

Dada uma superfície $S$ de curvatura média (ou gaussiana não nula) constante, é sempre possivel obtê-la por uma congruência de Guichard?

O Capítulo 5 é voltado ao tratamento dessa questão, provando que a mesma tem resposta afirmativa. Mais do que isto, toda superfície $S$ de curvatura constante pode ser obtida por uma família a três parâmetros de congruências de Guichard (Teoremas 5.2.3 e 5.3.3). Além disso, como cada uma destas congruências determina uma outra superfície $S^{s}$ (simétrica a $S$ com respeito a $S_{0}$ ) de curvatura constante igual a da $S$, pode-se concluir que:

A toda superfície $S$ de curvatura média (ou gaussiana não nula) constante podemos associar uma familia a três parâmetros de superfícies $S^{s}$ de mesma curvatura constante.

Desta forma, Bianchi obteve "novas" transformações entre superfícies de mesma curvatura constante, cuja interpretação analítica (dada em $[5,6]$ ) constitui um método para obter soluções das equações diferenciais estudadas no Capítulo 2, a partir de uma já conhecida.

Todas as demonstrações dos resultados apresentados na dissertação foram feitas utilizando o método do triedro móvel de E. Cartan [14], exposto no Capítulo 1.

O intento dos Apêndices A e B foi o de recordar alguns conceitos e resultados básicos da teoria local das superfícies de $\mathbb{R}^{3}$ e das formas diferenciais em $\mathbb{R}^{n}$, a fim de auxiliar o leitor na compreensão deste trabalho.

Como principais referências para o desenvolvimento desta dissertação utilizamos os livros da Professora K. Tenenblat [23, 24] e fizemos um estudo aprofundado dos artigos de L. Bianchi $[5,6]$. 


\section{Capítulo 1}

\section{O método do triedro móvel}

No Apêndice A desenvolvemos a teoria local das superfícies considerando um triedro "natural" constituído pelos campos de vetores $X_{u}, X_{v}, N$, associados a uma dada parametrização regular $X: U \subset \mathbb{R}^{2} \rightarrow \mathbb{R}^{3}$, que em geral não é ortonormal.

O intento deste primeiro capítulo é obter o estudo local das superfícies utilizando o "método do triedro móvel" de Elie Cartan (veja [14]), que consiste em escolher adequadamente um triedro ortonormal $\left\{e_{1}, e_{2}, e_{3}\right\}$ de forma tal que os campos $e_{1}, e_{2}$ são tangentes à superfície.

É importante ressaltar que, mesmo que na escolha destes triedros exista um certo grau de arbitrariedade, as entidades geométricas determinadas através deles independe da escolha feita (veja, por exemplo, a Proposição 1.3.2).

Para um estudo mais aprofundado do método do triedro móvel consulte, por exemplo, [11, $12,23]$.

\subsection{Triedro móvel e as formas de conexão}

Definição 1.1.1. Seja $X: U \subset \mathbb{R}^{2} \rightarrow S \subset \mathbb{R}^{3}$ uma superfície parametrizada regular e considere os campos de vetores $e_{i}: U \rightarrow \mathbb{R}^{3}, i=1,2,3$, tais que para cada ponto $q \in U$, os vetores $e_{1}(q), e_{2}(q), e_{3}(q)$ formam uma base ortonormal de $\mathbb{R}^{3}$. Então, para cada ponto $q \in U$, se $e_{1}(q), e_{2}(q)$ forem vetores tangentes à superfície $X$ em $q$ e, conseqüentemente, $e_{3}(q)$ for normal a $X$, a terna de campos de vetores $\left\{e_{1}, e_{2}, e_{3}\right\}$ é chamada de triedro (referencial) móvel associado à superfície $X$.

Note que, dada uma superfície parametrizada regular $X$, podemos sempre considerar o seguinte triedro móvel a ela associado:

$$
e_{3}=\frac{X_{u} \wedge X_{v}}{\left\|X_{u} \wedge X_{v}\right\|}, \quad e_{1}=\frac{X_{u}}{\left\|X_{u}\right\|}, \quad e_{2}=e_{3} \wedge e_{1}
$$

Seja $\left\{e_{1}, e_{2}, e_{3}\right\}$ um triedro móvel associado a $X: U \subset \mathbb{R}^{2} \rightarrow \mathbb{R}^{3}$, uma superfície parametrizada regular, e $q \in U$. Como $e_{1}(q), e_{2}(q)$ formam uma base para o plano tangente de $X$ em $q$, $\mathrm{T}_{X(q)} S=d X_{q}\left(\mathbb{R}^{2}\right)$, podemos escrever

$$
d X_{q}(v)=\left(\omega_{1}\right)_{q}(v) e_{1}(q)+\left(\omega_{2}\right)_{q}(v) e_{2}(q), \quad \forall v \in \mathbb{R}^{2},
$$

onde

$$
\left(\omega_{i}\right)_{q}(v)=\left\langle d X_{q}(v), e_{i}(q)\right\rangle, \quad i=1,2 .
$$


O conjunto das formas $\omega_{i}$ é dito co-referencial de $\left\{e_{i}\right\}, i=1,2,3$. Note que, se $v_{i} \in \mathbb{R}^{2}, i=1,2$, são tais que $d X_{q}\left(v_{i}\right)=e_{i}(q)$, então

$$
\left(\omega_{i}\right)_{q}\left(v_{j}\right)=\left\langle d X_{q}\left(v_{j}\right), e_{i}(q)\right\rangle=\left\langle e_{j}(q), e_{i}(q)\right\rangle=\delta_{j i}, \quad i, j=1,2 .
$$

Considerando a diferencial de $e_{i}: U \subset \mathbb{R}^{2} \rightarrow \mathbb{R}^{3}$ em $q \in U,\left(d e_{i}\right)_{q}: \mathbb{R}^{2} \rightarrow \mathbb{R}^{3}$, temos que $\left(d e_{i}\right)_{q}(v) \in \mathbb{R}^{3}$, onde $v \in \mathbb{R}^{2}$, pode ser escrito como combinação linear da base ortonormal de $\mathbb{R}^{3}$ dada por $\left\{e_{1}(q), e_{2}(q), e_{3}(q)\right\}$, isto é,

$$
\left(d e_{i}\right)_{q}(v)=\left(\omega_{i 1}\right)_{q}(v) e_{1}(q)+\left(\omega_{i 2}\right)_{q}(v) e_{2}(q)+\left(\omega_{i 3}\right)_{q}(v) e_{3}(q), \quad i=1,2,3,
$$

onde

$$
\left(\omega_{i j}\right)_{q}(v)=\left\langle\left(d e_{i}\right)_{q}(v), e_{j}(q)\right\rangle, \quad i, j=1,2,3 .
$$

Como $\left(d e_{i}\right)_{q}$ é uma aplicação linear, considerando $v, w \in \mathbb{R}^{2}$ e $\alpha \in \mathbb{R}$, segue que

$$
\begin{aligned}
\sum_{j=1}^{3}\left(\omega_{i j}\right)_{q}(\alpha v+w) e_{j}(q) & =\left(d e_{i}\right)_{q}(\alpha v+w)=\alpha\left(d e_{i}\right)_{q}(v)+\left(d e_{i}\right)_{q}(w) \\
& =\alpha \sum_{j=1}^{3}\left(\omega_{i j}\right)_{q}(v) e_{j}(q)+\sum_{j=1}^{3}\left(\omega_{i j}\right)_{q}(w) e_{j}(q) \\
& =\sum_{j=1}^{3}\left[\alpha\left(\omega_{i j}\right)_{q}(v)+\left(\omega_{i j}\right)_{q}(w)\right] e_{j}(q),
\end{aligned}
$$

ou seja, $\omega_{i j}, i, j=1,2,3$, são 1 -formas diferenciais em $U$, chamadas formas de conexão do triedro $\left\{e_{1}, e_{2}, e_{3}\right\}$. Para simplificar nossa notação, escreveremos:

$$
\begin{aligned}
\omega_{i} & =\left\langle d X, e_{i}\right\rangle, & i=1,2, \\
\omega_{j k} & =\left\langle d e_{j}, e_{k}\right\rangle, & j, k=1,2,3
\end{aligned}
$$

$\mathrm{e}$

$$
\begin{aligned}
d X & =\omega_{1} e_{1}+\omega_{2} e_{2}, \\
d e_{i} & =\omega_{i 1} e_{1}+\omega_{i 2} e_{2}+\omega_{i 3} e_{3}, \quad i=1,2,3 .
\end{aligned}
$$

Observação 1.1.2. A teoria que apresentaremos depende da escolha do triedro móvel, principalmente da escolha de $e_{1}$ e $e_{2}$. Considere, então, $X: U \subset \mathbb{R}^{2} \rightarrow \mathbb{R}^{3}$ uma superfície parametrizada regular e dois triedros móveis $\left\{e_{1}, e_{2}, e_{3}\right\}$ e $\left\{\bar{e}_{1}, \bar{e}_{2}, \bar{e}_{3}\right\}$ a ela associados, tais que $\bar{e}_{3}=e_{3}$ e

$$
\begin{aligned}
& \bar{e}_{1}=e_{1} \cos \theta+e_{2} \operatorname{sen} \theta, \\
& \bar{e}_{2}=-e_{1} \operatorname{sen} \theta+e_{2} \cos \theta,
\end{aligned}
$$

onde $\theta(u, v)$ é uma função real diferenciável que representa o ângulo entre os campos $e_{1}$ e $\bar{e}_{1}$. Então, seguem de (1.2), (1.3) e (1.4) as seguintes relações entre as 1-formas diferenciais associadas aos dois triedros:

$$
\begin{aligned}
\bar{\omega}_{1} & =\left\langle d X, \bar{e}_{1}\right\rangle=\omega_{1} \cos \theta+\omega_{2} \operatorname{sen} \theta \\
\bar{\omega}_{2} & =\left\langle d X, \bar{e}_{2}\right\rangle=-\omega_{1} \operatorname{sen} \theta+\omega_{2} \cos \theta \\
\bar{\omega}_{12} & =\left\langle d \bar{e}_{1}, \bar{e}_{2}\right\rangle=d \theta+\omega_{12}, \\
\bar{\omega}_{13} & =\left\langle d \bar{e}_{1}, \bar{e}_{3}\right\rangle=\omega_{13} \cos \theta+\omega_{23} \operatorname{sen} \theta \\
\bar{\omega}_{23} & =\left\langle d \bar{e}_{2}, \bar{e}_{3}\right\rangle=-\omega_{13} \operatorname{sen} \theta+\omega_{23} \cos \theta .
\end{aligned}
$$




\subsection{Equações de estrutura e Lema de E. Cartan}

Entre as 1-formas diferenciais $\omega_{1}, \omega_{2}, \omega_{i j}, i, j=1,2,3$, e suas diferenciais exteriores existem algumas relações, as quais veremos a seguir.

Teorema 1.2.1 (Equações de estrutura de E. Cartan). Sejam $X: U \subset \mathbb{R}^{2} \rightarrow \mathbb{R}^{3}$ uma superfície parametrizada regular, $\left\{e_{1}, e_{2}, e_{3}\right\}$ um triedro móvel associado a esta superfície e $\omega_{1}, \omega_{2}, \omega_{i j}$, $i, j=1,2,3$, as 1-formas associadas a este triedro definidas por (1.2). Então,

$$
\begin{array}{ll}
\omega_{i j}=-\omega_{j i}, & i, j=1,2,3, \\
d \omega_{i}=\omega_{j} \wedge \omega_{j i}, & i, j=1,2, \\
\omega_{1} \wedge \omega_{13}+\omega_{2} \wedge \omega_{23}=0, & \\
d \omega_{i j}=\sum_{k=1}^{3} \omega_{i k} \wedge \omega_{k j}, & i, j=1,2,3 .
\end{array}
$$

Demonstração. De $\left\langle e_{i}, e_{j}\right\rangle=\delta_{i j}$, temos que

$$
0=\left\langle d e_{i}, e_{j}\right\rangle+\left\langle e_{i}, d e_{j}\right\rangle=\omega_{i j}+\omega_{j i}
$$

isto é, vale a (1.5). Agora, usando (1.3) e (1.4), resulta

$$
\begin{aligned}
0=d(d X) & =e_{1} d \omega_{1}+d e_{1} \wedge \omega_{1}+e_{2} d \omega_{2}+d e_{2} \wedge \omega_{2} \\
& =e_{1} d \omega_{1}+\left(\omega_{12} e_{2}+\omega_{13} e_{3}\right) \wedge \omega_{1}+e_{2} d \omega_{2}+\left(\omega_{21} e_{1}+\omega_{23} e_{3}\right) \wedge \omega_{2}
\end{aligned}
$$

de onde obtemos

$$
e_{1}\left(d \omega_{1}+\omega_{21} \wedge \omega_{2}\right)+e_{2}\left(\omega_{12} \wedge \omega_{1}+d \omega_{2}\right)+e_{3}\left(\omega_{13} \wedge \omega_{1}+\omega_{23} \wedge \omega_{2}\right)=0
$$

Sendo $\left\{e_{1}, e_{2}, e_{3}\right\}$ campos linearmente independentes, seguem as relações (1.6) e (1.7). Da mesma maneira, usando a equação (1.4), temos

$$
0=d\left(d e_{i}\right)=d\left(\sum_{j=1}^{3} e_{j} \omega_{i j}\right)=\sum_{j=1}^{3}\left[\sum_{k=1}^{3} \omega_{k j} \wedge \omega_{i k}+d \omega_{i j}\right] e_{j}, \quad i=1,2,3,
$$

de onde resulta (1.8).

As equações de (1.6) a (1.8) são chamadas de equações de estrutura de E. Cartan [14].

Observe que, para cada $q \in U$, como $\left\{X_{u}(q), X_{v}(q)\right\}$ e $\left\{e_{1}(q), e_{2}(q)\right\}$ são bases para o plano tangente à superfície $X$ em $q$, podemos escrever

$$
X_{u}=a_{1} e_{1}+a_{2} e_{2}, \quad X_{v}=b_{1} e_{1}+b_{2} e_{2},
$$

onde $a_{i}, b_{i}: U \rightarrow \mathbb{R}, i=1,2$, são funções diferenciáveis tais que $\left|\begin{array}{ll}a_{1} & a_{2} \\ b_{1} & b_{2}\end{array}\right| \neq 0$. Logo, segue de $d X=X_{u} d u+X_{v} d v$ que

$$
d X=\left(a_{1} d u+b_{1} d v\right) e_{1}+\left(a_{2} d u+b_{2} d v\right) e_{2}
$$


de onde obtemos, comparando com (1.3),

$$
\omega_{1}=a_{1} d u+b_{1} d v, \quad \omega_{2}=a_{2} d u+b_{2} d v .
$$

Como

$$
\omega_{1} \wedge \omega_{2}=\left|\begin{array}{ll}
a_{1} & b_{1} \\
a_{2} & b_{2}
\end{array}\right| d u \wedge d v \neq 0,
$$

$\omega_{1}$ e $\omega_{2}$ são 1-formas linearmente independentes (ver Proposição B.1.5). Portanto, toda 1-forma diferencial em $U$ é uma combinação linear de $\omega_{1}$ e $\omega_{2}$. Usando este fato e o Teorema 1.2.1, podemos provar o próximo resultado.

Proposição 1.2.2 (Lema de Cartan). Se $\omega_{1}, \omega_{2}$ são 1-formas diferenciais, então existe uma única 1-forma diferencial $\omega_{12}$ que satisfaz

$$
\begin{aligned}
& d \omega_{1}=\omega_{2} \wedge \omega_{21}, \\
& d \omega_{2}=\omega_{1} \wedge \omega_{12} .
\end{aligned}
$$

Demonstração. Suponha que existam duas 1-formas diferenciais, $\omega_{12}$ e $\bar{\omega}_{12}$, tais que satisfaçam as relações acima. Vamos mostrar que $\omega_{12}=\bar{\omega}_{12}$. Para isso, da primeira relação temos

$$
d \omega_{1}=\omega_{2} \wedge \omega_{21} \quad \text { e } \quad d \omega_{1}=\omega_{2} \wedge \bar{\omega}_{21}
$$

e, portanto, subtraindo estas

$$
\omega_{2} \wedge\left(\omega_{21}-\bar{\omega}_{21}\right)=0 .
$$

Analogamente, da segunda relação obtemos $\omega_{1} \wedge\left(\omega_{12}-\bar{\omega}_{12}\right)=0$.

Como $\omega_{12}-\bar{\omega}_{12}=-\left(\omega_{21}-\bar{\omega}_{21}\right)$ e estas são 1-formas diferenciais, são então combinações lineares das 1-formas $\omega_{1}$ e $\omega_{2}$. Assim, podemos escrever

$$
\omega_{12}-\bar{\omega}_{12}=A \omega_{1}+B \omega_{2},
$$

onde $A$ e $B$ são funções a valores reais. Logo, de

$$
\omega_{1} \wedge\left(A \omega_{1}+B \omega_{2}\right)=0 \quad \text { e } \quad \omega_{2} \wedge\left(A \omega_{1}+B \omega_{2}\right)=0
$$

segue que $A=B=0$ e, portanto, $\omega_{12}=\bar{\omega}_{12}$.

Por fim, observe que escrevendo as 1-formas $\omega_{13}$ e $\omega_{23}$ na seguinte maneira:

$$
\omega_{13}=h_{11} \omega_{1}+h_{12} \omega_{2}, \quad \omega_{23}=h_{21} \omega_{1}+h_{22} \omega_{2},
$$

onde $h_{i j}: U \subset \mathbb{R}^{2} \rightarrow \mathbb{R}, i, j=1,2$, são funções diferenciáveis, de (1.7) obtemos

$$
0=\omega_{1} \wedge\left(h_{11} \omega_{1}+h_{12} \omega_{2}\right)+\omega_{2} \wedge\left(h_{21} \omega_{1}+h_{22} \omega_{2}\right)=\left(h_{12}-h_{21}\right) \omega_{1} \wedge \omega_{2},
$$

ou seja, $h_{12}=h_{21}$.

\subsection{Alguns resultados importantes utilizando o método do triedro móvel}

Com base nas seções anteriores, vamos desenvolver a teoria local das superfícies em $\mathbb{R}^{3}$ utilizando um triedro móvel e as 1-formas diferenciais associadas a este triedro. 


\subsubsection{A primeira e a segunda formas quadráticas}

Seja $X: U \subset \mathbb{R}^{2} \rightarrow S \subset \mathbb{R}^{3}$ uma superfície parametrizada regular, $\left\{e_{1}, e_{2}, e_{3}\right\}$ um triedro móvel associado a $X$ e $\omega_{1}, \omega_{2}, \omega_{i j}, i, j=1,2,3$, as 1 -formas diferenciais definidas por (1.2) associadas a este triedro.

A primeira forma quadrática $\mathrm{I}_{q}$ em $q \in U$ é uma aplicação que, a cada $w=d X_{q}(v) \in \mathrm{T}_{X(q)} S$, associa $\mathrm{I}_{q}(w)=\|w\|^{2}$. Portanto, usando (1.3), temos

$$
\begin{aligned}
\mathrm{I}_{q}(w) & =\left\|d X_{q}(v)\right\|^{2}=\left\|\left(\omega_{1}\right)_{q}(v) e_{1}(q)+\left(\omega_{2}\right)_{q}(v) e_{2}(q)\right\|^{2} \\
& =\left(\omega_{1}\right)_{q}^{2}(v)+\left(\omega_{2}\right)_{q}^{2}(v),
\end{aligned}
$$

isto é,

$$
\mathrm{I}=\omega_{1}^{2}+\omega_{2}^{2}
$$

Da mesma forma, a segunda forma quadrática $\mathrm{II}_{q}$ é uma aplicação que para cada $w \in \mathrm{T}_{X(q)} S$, $w=d X_{q}(v)$, associa $\mathrm{II}_{q}(w)=-\left\langle d X_{q}(v),\left(d e_{3}\right)_{q}(v)\right\rangle$, pois $e_{3}$ é um campo normal à superfície $X$. Assim,

$$
\begin{aligned}
\operatorname{II}_{q}(w) & =-\left\langle\left(\omega_{1}\right)_{q}(v) e_{1}(q)+\left(\omega_{2}\right)_{q}(v) e_{2}(q),\left(\omega_{31}\right)_{q}(v) e_{1}(q)+\left(\omega_{32}\right)_{q}(v) e_{2}(q)\right\rangle \\
& =\left(\omega_{1}\right)_{q}(v)\left(\omega_{13}\right)_{q}(v)+\left(\omega_{2}\right)_{q}(v)\left(\omega_{23}\right)_{q}(v),
\end{aligned}
$$

ou seja,

$$
\mathrm{II}=\omega_{1} \omega_{13}+\omega_{2} \omega_{23} .
$$

Considerando $\omega_{13}$ e $\omega_{23}$ como em (1.10), temos

$$
\mathrm{II}=h_{11} \omega_{1}^{2}+h_{12}\left(\omega_{1} \omega_{2}+\omega_{2} \omega_{1}\right)+h_{22} \omega_{2}^{2} .
$$

Vamos, agora, provar um importante resultado.

Proposição 1.3.1. Sejam $X: U \subset \mathbb{R}^{2} \rightarrow \mathbb{R}^{3}$ uma superfície parametrizada regular, $q \in U$, $\left\{e_{1}, e_{2}, e_{3}\right\}$ um triedro móvel associado a $X$ e $\omega_{1}, \omega_{2}, \omega_{i j}, i, j=1,2,3$, as 1-formas associadas a este triedro. Então, o vetor $w=d X_{q}(v) \in T_{X(q)} S, v \in \mathbb{R}^{2}$,

(i) é uma direção principal de $X$ em q se, e só se,

$$
\left(\omega_{1}\right)_{q}(v)\left(\omega_{23}\right)_{q}(v)-\left(\omega_{2}\right)_{q}(v)\left(\omega_{13}\right)_{q}(v)=0 ;
$$

(ii) é uma direção assintótica de $X$ em q se, e somente se,

$$
\left(\omega_{1}\right)_{q}(v)\left(\omega_{13}\right)_{q}(v)+\left(\omega_{2}\right)_{q}(v)\left(\omega_{23}\right)_{q}(v)=0 .
$$

Demonstração. (i) Escrevendo $w=a X_{u}(q)+b X_{v}(q), a, b \in \mathbb{R}$, a prova segue da Proposição A.3.6 e da igualdade:

$$
\begin{aligned}
\left(\omega_{1}\right. & \left.\wedge \omega_{2}\right)_{q}\left(\frac{\partial}{\partial u}, \frac{\partial}{\partial v}\right)\left[\left(\omega_{1}\right)_{q}(v)\left(\omega_{23}\right)_{q}(v)-\left(\omega_{2}\right)_{q}(v)\left(\omega_{13}\right)_{q}(v)\right] \\
= & b^{2}(F g-f G)(q)+a b(E g-e G)(q)+a^{2}(E f-e F)(q) .
\end{aligned}
$$

(ii) A prova segue direto de $\mathrm{II}_{q}(v)=0$ e de (1.12). 


\subsubsection{As equações de Gauss e Codazzi-Mainardi}

Note que, de (1.11), (1.13) e da Proposição A.3.6, as curvaturas principais $\kappa_{1}$ e $\kappa_{2}$ da superfície $X$ são soluções de

$$
\left|\begin{array}{cc}
h_{11}-\kappa & h_{12} \\
h_{12} & h_{22}-\kappa
\end{array}\right|=0,
$$

isto é,

$$
\kappa^{2}-\left(h_{11}+h_{22}\right) \kappa+h_{11} h_{22}-h_{12}^{2}=0 .
$$

Portanto, comparando com (A.8), obtemos que as curvaturas média e gaussiana da superfície $X$ são dadas por:

$$
\begin{aligned}
\mathrm{H} & =\frac{1}{2}\left(h_{11}+h_{22}\right), \\
K & =h_{11} h_{22}-h_{12}^{2} .
\end{aligned}
$$

A seguir apresentaremos um resultado que mostra como $\mathrm{H}$ e $K$ podem ser obtidas por meio das formas diferenciais associadas a um triedro móvel.

Proposição 1.3.2. Seja $X$ uma superfície parametrizada regular e $\left\{e_{1}, e_{2}, e_{3}\right\}$ um triedro móvel a ela associado. Então valem as seguintes equações

$$
\begin{aligned}
& d \omega_{12}=-\omega_{13} \wedge \omega_{23}=-K \omega_{1} \wedge \omega_{2}, \\
& \omega_{1} \wedge \omega_{23}+\omega_{13} \wedge \omega_{2}=2 H \omega_{1} \wedge \omega_{2} .
\end{aligned}
$$

Demonstração. Substituindo as expressões de $\omega_{13}$ e $\omega_{23}$, dadas em (1.10), na equação de estrutura $d \omega_{12}=-\omega_{13} \wedge \omega_{23}$ resulta que

$$
d \omega_{12}=-\left(h_{11} h_{22}-h_{12}^{2}\right) \omega_{1} \wedge \omega_{2}=-K \omega_{1} \wedge \omega_{2} .
$$

Da mesma forma, usando (1.10) e (1.16), temos

$$
\omega_{1} \wedge \omega_{23}+\omega_{13} \wedge \omega_{2}=\left(h_{11}+h_{22}\right) \omega_{1} \wedge \omega_{2}=2 \mathrm{H} \omega_{1} \wedge \omega_{2}
$$

A equação (1.18) é conhecida como equação de Gauss e as relações dadas por (1.8):

$$
\begin{aligned}
& d \omega_{13}=\omega_{12} \wedge \omega_{23}, \\
& d \omega_{23}=\omega_{21} \wedge \omega_{13},
\end{aligned}
$$

como equações de Codazzi-Mainardi.

O próximo resultado relaciona o método do triedro móvel ao tratamento clássico dado às superfícies em $\mathbb{R}^{3}$.

Proposição 1.3.3. Seja $X: U \subset \mathbb{R}^{2} \rightarrow \mathbb{R}^{3}$ uma superfície parametrizada regular cujas curvas coordenadas são ortogonais. Denote os coeficientes da primeira forma quadrática por $E, F, G$, os da segunda forma quadrática por e, $f, g$, e considere o triedro móvel associado a $X$ dado por:

$$
e_{1}=\frac{X_{u}}{\sqrt{E}}, \quad e_{2}=\frac{X_{v}}{\sqrt{G}}, \quad e_{3}=e_{1} \wedge e_{2} .
$$


Então, temos as seguintes relações

$$
\begin{aligned}
\omega_{1} & =\sqrt{E} d u, \quad \omega_{2}=\sqrt{G} d v \\
\omega_{12} & =-\frac{(\sqrt{E})_{v}}{\sqrt{G}} d u+\frac{(\sqrt{G})_{u}}{\sqrt{E}} d v \\
\omega_{13} & =\frac{1}{\sqrt{E}}(e d u+f d v), \\
\omega_{23} & =\frac{1}{\sqrt{G}}(f d u+g d v) .
\end{aligned}
$$

Demonstração. Como $d X=X_{u} d u+X_{v} d v$ e as curvas coordenadas são ortogonais (isto é, $F=0$ ), temos que

$$
\begin{aligned}
& \omega_{1}=\left\langle d X, e_{1}\right\rangle=\sqrt{E} d u, \\
& \omega_{2}=\left\langle d X, e_{2}\right\rangle=\sqrt{G} d v .
\end{aligned}
$$

Escrevendo $\omega_{12}=a d u+b d v$, da Proposição 1.2.2 e das relações (1.20) segue que

$$
\begin{gathered}
-(\sqrt{E})_{v} d u \wedge d v=a \sqrt{G} d u \wedge d v \\
(\sqrt{G})_{u} d u \wedge d v=b \sqrt{E} d u \wedge d v
\end{gathered}
$$

de onde resulta que

$$
\omega_{12}=-\frac{(\sqrt{E})_{v}}{\sqrt{G}} d u+\frac{(\sqrt{G})_{u}}{\sqrt{E}} d v
$$

As equações (1.22) e (1.23) são facilmente obtidas substituindo nas relações

$$
\omega_{13}=\left\langle d e_{1}, e_{3}\right\rangle \quad \text { e } \quad \omega_{23}=\left\langle d e_{2}, e_{3}\right\rangle,
$$

as expressões de $e_{1}$ e $e_{2}$.

Note que, usando (1.20) e (1.21), resulta da equação de Gauss, $d \omega_{12}=-K \omega_{1} \wedge \omega_{2}$, que

$$
\left[\left(\frac{(\sqrt{E})_{v}}{\sqrt{G}}\right)_{v}+\left(\frac{(\sqrt{G})_{u}}{\sqrt{E}}\right)_{u}\right] d u \wedge d v=-K \sqrt{E G} d u \wedge d v
$$

isto é, re-obtemos a equação (A.9):

$$
K=-\frac{1}{\sqrt{E G}}\left[\left(\frac{(\sqrt{E})_{v}}{\sqrt{G}}\right)_{v}+\left(\frac{(\sqrt{G})_{u}}{\sqrt{E}}\right)_{u}\right] .
$$

Da mesma forma, de (1.22) e (1.23), obtemos que as equações de Codazzi-Mainardi equivalem a (A.10) e (A.11):

$$
\begin{aligned}
& 2 E G\left(e_{v}-f_{u}\right)-(E g+G e) E_{v}-f\left(E G_{u}-G E_{u}\right)=0 \\
& 2 E G\left(f_{v}-g_{u}\right)+(E g+G e) G_{u}-f\left(E G_{v}-G E_{v}\right)=0 .
\end{aligned}
$$


Exemplo 1.3.4. Seja $X(u, v)=(h(u) \cos v, h(u) \operatorname{sen} v, k(u))$ a parametrização de uma superfície de revolução gerada pela rotação da curva $\alpha(u)=(h(u), 0, k(u))$ ao redor do eixo $z$, onde $h$ e $k$ são funções a valores reais tais que $\left(h^{\prime}\right)^{2}+\left(k^{\prime}\right)^{2} \neq 0$ e $h>0$. Os coeficientes da primeira e da segunda formas quadráticas de $X$ são

$$
\begin{array}{lll}
E=\left(h^{\prime}\right)^{2}+\left(k^{\prime}\right)^{2}, & F=0, & G=h^{2} ; \\
e=\frac{k^{\prime \prime} h^{\prime}-k^{\prime} h^{\prime \prime}}{\sqrt{\left(h^{\prime}\right)^{2}+\left(k^{\prime}\right)^{2}},} & f=0, & g=\frac{h k^{\prime}}{\sqrt{\left(h^{\prime}\right)^{2}+\left(k^{\prime}\right)^{2}}} .
\end{array}
$$

Como as curvas coordenadas de $X$ são ortogonais, considerando o triedro móvel

$$
e_{1}=\frac{X_{u}}{\sqrt{\left(h^{\prime}\right)^{2}+\left(k^{\prime}\right)^{2}}}, \quad e_{2}=\frac{X_{v}}{h}, \quad e_{3}=e_{1} \wedge e_{2}
$$

segue da Proposição 1.3.3 que as 1-formas associadas a este triedro são:

$$
\begin{aligned}
\omega_{1} & =\sqrt{\left(h^{\prime}\right)^{2}+\left(k^{\prime}\right)^{2}} d u, \\
\omega_{2} & =h d v, \\
\omega_{12} & =\frac{h^{\prime}}{\sqrt{\left(h^{\prime}\right)^{2}+\left(k^{\prime}\right)^{2}}} d v, \\
\omega_{13} & =\frac{k^{\prime \prime} h^{\prime}-k^{\prime} h^{\prime \prime}}{\left(h^{\prime}\right)^{2}+\left(k^{\prime}\right)^{2}} d u, \\
\omega_{23} & =\frac{k^{\prime}}{\sqrt{\left(h^{\prime}\right)^{2}+\left(k^{\prime}\right)^{2}}} d v .
\end{aligned}
$$

Definição 1.3.5. Triedros $\left\{e_{1}, e_{2}, e_{3}\right\}$ (como o do Exemplo 1.3.4) onde $e_{1}, e_{2}$ são campos de vetores principais são ditos triedros móveis principais.

Temos o seguinte.

Proposição 1.3.6. Sejam $X: U \subset \mathbb{R}^{2} \rightarrow \mathbb{R}^{3}$ uma superfície parametrizada regular sem pontos umbílicos e considere $\left\{e_{1}, e_{2}, e_{3}\right\}$ um triedro móvel principal. Então as curvaturas principais $\kappa_{1}, \kappa_{2}$ de $X$ em $q \in U$ são tais que

$$
\omega_{13}=\kappa_{1} \omega_{1}, \quad \omega_{23}=\kappa_{2} \omega_{2} .
$$

Demonstração. Da Proposição A.5.3, podemos supor que as curvas coordenadas de $X$ são linhas de curvatura. Logo, da Proposição A.5.4, temos que $F=0=f$ e que suas curvaturas principais são $\kappa_{1}=\frac{e}{E}$ e $\kappa_{2}=\frac{g}{G}$. Assim, associando à superfície $X$ o triedro móvel principal

$$
e_{1}=\frac{X_{u}}{\sqrt{E}}, \quad e_{2}=\frac{X_{v}}{\sqrt{G}}, \quad e_{3}=e_{1} \wedge e_{2},
$$

resultam, de (1.20), (1.22) e (1.23), as relações (1.28).

Obteremos, agora, as equações de Codazzi-Mainardi de uma superfície cujas curvas coordenadas são linhas de curvatura. 
Proposição 1.3.7. Seja $X: U \subset \mathbb{R}^{2} \rightarrow \mathbb{R}^{3}$ uma superfície parametrizada regular sem pontos umbilicos, cujas curvas coordenadas são linhas de curvatura. Denotando por $\kappa_{1}, \kappa_{2}$ as curvaturas principais de $X$, temos que as equações de Codazzi-Mainardi são dadas por:

$$
\frac{1}{\kappa_{1}-\kappa_{2}} \frac{\partial \kappa_{1}}{\partial v}=-\frac{\partial(\log \sqrt{E})}{\partial v}, \quad \frac{1}{\kappa_{2}-\kappa_{1}} \frac{\partial \kappa_{2}}{\partial u}=-\frac{\partial(\log \sqrt{G})}{\partial u}
$$

Demonstração. Apresentaremos apenas a prova da primeira das equações acima.

Considerando o triedro móvel principal associado a $X$ :

$$
e_{1}=\frac{X_{u}}{\sqrt{E}}, \quad e_{2}=\frac{X_{v}}{\sqrt{G}}, \quad e_{3}=e_{1} \wedge e_{2},
$$

decorre da Proposição 1.3.6 que $\omega_{i 3}=\kappa_{i} \omega_{i}, i=1,2$. Portanto, a equação de Codazzi-Mainardi $d \omega_{13}=\omega_{12} \wedge \omega_{23}$ pode ser escrita como

$$
d \kappa_{1} \wedge \omega_{1}+\kappa_{1} d \omega_{1}=d \omega_{13}=\kappa_{2} \omega_{12} \wedge \omega_{2}
$$

de onde, usando a equação de estrutura $d \omega_{1}=\omega_{2} \wedge \omega_{21}$, resulta:

$$
d \kappa_{1} \wedge \omega_{1}=\left(\kappa_{2}-\kappa_{1}\right) \omega_{12} \wedge \omega_{2} .
$$

Assim, segue de (1.20) e (1.21) que

$$
\sqrt{E} \frac{\partial \kappa_{1}}{\partial v} d v \wedge d u=-\left(\kappa_{2}-\kappa_{1}\right)(\sqrt{E})_{v} d u \wedge d v
$$

que equivale à primeira equação de (1.29).

Como último resultado, apresentaremos a seguinte caracterização de superfícies parametrizadas regulares:

Proposição 1.3.8. Seja $X: U \subset \mathbb{R}^{2} \rightarrow \mathbb{R}^{3}$ uma aplicação diferenciável. Então $X$ é uma superfície parametrizada regular se, e somente se, existem funções diferenciáveis $f_{1}, f_{2}: U \rightarrow \mathbb{R}^{3}$ e 1-formas diferenciais $\phi_{1}, \phi_{2}$ em $U$ tais que:

(i) para todo $q \in U, f_{1}(q)$ e $f_{2}(q)$ são vetores linearmente independentes de $\mathbb{R}^{3}$;

(ii) $\phi_{1}$ e $\phi_{2}$ são 1-formas linearmente independentes;

(iii) para todo $q \in U, d X_{q}=\left(\phi_{1}\right)_{q} f_{1}(q)+\left(\phi_{2}\right)_{q} f_{2}(q)$.

Neste caso, $f_{1}$ e $f_{2}$ são campos tangentes à superfície $X$.

Demonstração. Se $X(u, v)$ é uma superfície parametrizada regular, então, para todo $q \in U$, temos $d X_{q}=X_{u} d u+X_{v} d v$, onde $X_{u}, X_{v}$ é base do plano tangente de $X$ em $q$ e $d u, d v$ são 1-formas linearmente independentes. Portanto, as condições da proposição estão satisfeitas.

Por outro lado, supondo que $f_{1}, f_{2}, \phi_{1}, \phi_{2}$ satisfazem as condições da proposição, vamos mostrar que $d X_{q}$ é injetiva, para todo $q \in U$. Assim mostrado, segue que $X$ é uma superfície parametrizada regular. Para isso, considere $v \in \mathbb{R}^{2}$ tal que $d X_{q}(v)=0$, isto é,

$$
\left(\phi_{1}\right)_{q}(v) f_{1}(q)+\left(\phi_{2}\right)_{q}(v) f_{2}(q)=0 .
$$


Do item $(i)$, concluímos que $\left(\phi_{1}\right)_{q}(v)=\left(\phi_{2}\right)_{q}(v)=0$. Portanto, para qualquer $w \in \mathbb{R}^{2}$, temos

$$
\left(\phi_{1} \wedge \phi_{2}\right)_{q}(v, w)=\left(\phi_{1}\right)_{q}(v)\left(\phi_{2}\right)_{q}(w)-\left(\phi_{2}\right)_{q}(v)\left(\phi_{1}\right)_{q}(w)=0 .
$$

Em particular, supondo $v \neq 0$, podemos escolher $w$ de tal maneira que $v$ e $w$ formam uma base para $\mathbb{R}^{2}$. Da bi-linearidade de $\left(\phi_{1} \wedge \phi_{2}\right)_{q}$, concluímos que $\left(\phi_{1} \wedge \phi_{2}\right)_{q} \equiv 0$, o que é um absurdo pois $\phi_{1}, \phi_{2}$ são 1-formas linearmente independentes. Logo, $v=0$.

Também, de (iii) temos que, para todo $w \in \mathrm{T}_{X(q)} S$, existe $v \in \mathbb{R}^{2}$ tal que

$$
w=d X_{q}(v)=\left(\phi_{1}\right)_{q}(v) f_{1}(q)+\left(\phi_{2}\right)_{q}(v) f_{2}(q) .
$$

Assim, de $(i)$ segue que $f_{1}(q)$ e $f_{2}(q)$ geram o plano tangente de $X$ em $q$. 


\section{Capítulo 2}

\section{Equações diferenciais associadas a superfícies de curvatura constante}

Neste capítulo apresentaremos equações diferenciais parciais, não lineares, de segunda ordem, cujas soluções estão em correspondência com superfícies de curvatura constante. Mostraremos que superfícies de tal tipo, quando parametrizadas por linhas de curvatura, estão em correspondência com soluções de certas equações diferenciais.

Em particular, as superfícies de curvatura gaussiana constante positiva (assim como as de curvatura média constante não nula) estão associadas a soluções da equação de Sine-Gordon elíptica (Proposição 2.2.1 e Observação 2.1.2), as pseudo-esféricas (i.e. de curvatura constante negativa) associam-se a soluções da equação de Sine-Gordon (Proposição 2.3.3) e as superfícies mínimas, a soluções da equação de Liouville (Corolário 2.1.3).

Com base nesta associação, daremos uma interpretação geométrica das soluções da equação de Sine-Gordon (respectivamente, de Sine-Gordon elíptica) em termos das direções assintóticas (respectivamente, conjugadas) das superfícies pseudo-esféricas (respectivamente, de curvatura gaussiana constante positiva) a elas associadas (veja as Proposições 2.2.2 e 2.3.4).

Lembramos, assim, da seguinte:

Definição 2.0.9. Seja $\phi(u, v)$ uma função real diferenciável definida em um aberto de $\mathbb{R}^{2}$. Denotaremos por $\Delta \phi$ o laplaciano de $\phi$, isto é,

$$
\Delta \phi=\phi_{u u}+\phi_{v v}
$$

\subsection{Superfícies de curvatura média constante}

Proposição 2.1.1. A toda superfície de curvatura média constante $\mathrm{H}$, sem pontos umbílicos, podemos associar uma solução $\phi$ de cada equação

$$
\Delta \phi=C^{2} e^{-2 \phi}-\mathrm{H}^{2} e^{2 \phi},
$$

onde C é uma constante não nula. Por outro lado, a cada solução da equação (2.1), a menos de movimentos rígidos de $\mathbb{R}^{3}$, podemos associar um par de superfícies de curvatura média constante e igual $a \mathrm{H}$. 
Demonstração. Seja $X: U \subset \mathbb{R}^{2} \rightarrow \mathbb{R}^{3}$ uma superfície de curvatura média constante $\mathrm{H}$, sem pontos umbílicos. Supondo que as curvas coordenadas de $X$ são linhas de curvatura, temos que as formas quadráticas desta superfície são dadas por (veja Proposições A.5.3 e A.5.4):

$$
\begin{aligned}
\mathrm{I} & =E d u^{2}+G d v^{2}, \\
\mathrm{II} & =e d u^{2}+g d v^{2} .
\end{aligned}
$$

Além disso, pela Proposição 1.3.7, as curvaturas principais $\kappa_{1}=\frac{e}{E}$ e $\kappa_{2}=\frac{g}{G}$ satisfazem as equações de Codazzi-Mainardi:

$$
\left\{\begin{array}{c}
\frac{1}{\kappa_{1}-\kappa_{2}} \frac{\partial \kappa_{1}}{\partial v}=-\frac{\partial(\log \sqrt{E})}{\partial v} \\
\frac{1}{\kappa_{2}-\kappa_{1}} \frac{\partial \kappa_{2}}{\partial u}=-\frac{\partial(\log \sqrt{G})}{\partial u}
\end{array}\right.
$$

Como $\kappa_{1}+\kappa_{2}=2 \mathrm{H}$, podemos escrever $\kappa_{1}-\kappa_{2}=2\left(\kappa_{1}-\mathrm{H}\right)$ e $\kappa_{2}-\kappa_{1}=2\left(\kappa_{2}-\mathrm{H}\right)$ e, assim, as equações acima se reduzem a

$$
\left\{\begin{array}{l}
\frac{1}{\kappa_{1}-\mathrm{H}} \frac{\partial \kappa_{1}}{\partial v}=-\frac{\partial(\log E)}{\partial v} \\
\frac{1}{\kappa_{2}-\mathrm{H}} \frac{\partial \kappa_{1}}{\partial u}=-\frac{\partial(\log G)}{\partial u} .
\end{array}\right.
$$

Segue da hipótese de $X$ não ter pontos umbílicos que

$$
\left(\kappa_{1}-\mathrm{H}\right)\left(\kappa_{2}-\mathrm{H}\right)=K-\mathrm{H}^{2}<0 .
$$

Então, podemos supor que $\kappa_{1}-\mathrm{H}>0$ e $\kappa_{2}-\mathrm{H}<0$ e, assim,

$$
\left\{\begin{aligned}
\frac{\partial}{\partial v}\left(\log \left(E\left(\kappa_{1}-\mathrm{H}\right)\right)\right) & =0 \\
\frac{\partial}{\partial u}\left(\log \left(-G\left(\kappa_{2}-\mathrm{H}\right)\right)\right) & =0
\end{aligned}\right.
$$

ou seja, existem funções positivas $\psi_{1}(u)$ e $\psi_{2}(v)$ tais que

$$
\left\{\begin{aligned}
E\left(\kappa_{1}-\mathrm{H}\right) & =\psi_{1}(u), \\
-G\left(\kappa_{2}-\mathrm{H}\right) & =\psi_{2}(v) .
\end{aligned}\right.
$$

Então, fixada uma constante $C \neq 0$, a menos de uma reparametrização ${ }^{1}$ de $X$, podemos supor que

$$
\left\{\begin{aligned}
E\left(\kappa_{1}-\mathrm{H}\right) & =|C|, \\
-G\left(\kappa_{2}-\mathrm{H}\right) & =|C| .
\end{aligned}\right.
$$

\footnotetext{
${ }^{1}$ Como $\psi_{1}$ é função somente de $u$ e $\psi_{2}$ é função somente de $v$, podemos definir duas funções $p$ e $q$ pondo

$$
p(u)=\frac{1}{\bar{c}} \int \sqrt{\psi_{1}(u)} d u, \quad q(v)=\frac{1}{\bar{c}} \int \sqrt{\psi_{2}(v)} d v, \quad \text { onde } \quad \bar{c}=\sqrt{|C|} .
$$

Como $p^{\prime}$ e $q^{\prime}$ não se anulam, pelo Teorema da Função Inversa, existem as inversas $u=u(p)$ e $v=v(q)$ de $p$ e $q$, respectivamente. Definindo $\bar{X}(p, q)=X(u(p), v(q))$, os coeficientes da primeira forma quadrática de $\bar{X}$ são dados
} 
Portanto, como $\kappa_{1}-\mathrm{H}=-\left(\kappa_{2}-\mathrm{H}\right)$, concluímos que $E=G$. Seja, então, $\phi(u, v)$ uma função diferenciável tal que $E=G=e^{2 \phi}$. Segue de (2.4) que

$$
\begin{aligned}
& \kappa_{1}=\mathrm{H}+|C| e^{-2 \phi}, \\
& \kappa_{2}=\mathrm{H}-|C| e^{-2 \phi} .
\end{aligned}
$$

Logo, da equação de Gauss (1.25), temos

$$
\begin{aligned}
K & =-\frac{1}{\sqrt{E G}}\left[\left(\frac{(\sqrt{E})_{v}}{\sqrt{G}}\right)_{v}+\left(\frac{(\sqrt{G})_{u}}{\sqrt{E}}\right)_{u}\right] \\
& =-\frac{1}{e^{2 \phi}}\left(\phi_{v v}+\phi_{u u}\right)=-\frac{1}{e^{2 \phi}} \Delta \phi .
\end{aligned}
$$

Por outro lado,

$$
K=\kappa_{1} \kappa_{2}=\mathrm{H}^{2}-C^{2} e^{-4 \phi}
$$

Portanto,

$$
\Delta \phi=C^{2} e^{-2 \phi}-\mathrm{H}^{2} e^{2 \phi},
$$

ou seja, $\phi$ é solução de (2.1).

Observe que se considerarmos $\kappa_{1}-\mathrm{H}<0$ e $\kappa_{2}-\mathrm{H}>0$, com argumentos análogos, obtemos $E=G=e^{2 \phi} \mathrm{e}$, assim, $\kappa_{1}=\mathrm{H}-|C| e^{-2 \phi}$ e $\kappa_{2}=\mathrm{H}+|C| e^{-2 \phi}$. Portanto, $\phi$ é solução da mesma equação.

Reciprocamente, se $\phi$ for solução da equação (2.1), onde $C \neq 0$ e H são constantes, considere as formas quadráticas

$$
\begin{aligned}
\mathrm{I} & =e^{2 \phi}\left(d u^{2}+d v^{2}\right), \\
\mathrm{II} & =\left(\mathrm{H} e^{2 \phi}+C\right) d u^{2}+\left(\mathrm{H} e^{2 \phi}-C\right) d v^{2} .
\end{aligned}
$$

Note que

$$
K=\frac{e g-f^{2}}{E G-F^{2}}=\mathrm{H}^{2}-C^{2} e^{-4 \phi}
$$

e, como,

$$
-\frac{1}{\sqrt{E G}}\left[\left(\frac{(\sqrt{E})_{v}}{\sqrt{G}}\right)_{v}+\left(\frac{(\sqrt{G})_{u}}{\sqrt{E}}\right)_{u}\right]=-\frac{1}{e^{2 \phi}} \Delta \phi=\mathrm{H}^{2}-C^{2} e^{-4 \phi},
$$

concluímos que os coeficientes destas formas quadráticas satisfazem a equação de Gauss. Da mesma maneira vemos que

$$
\begin{aligned}
& 2 E G\left(e_{v}-f_{u}\right)-(E g+G e) E_{v}-f\left(E G_{u}-G E_{u}\right) \\
& \quad=4 \mathrm{H} e^{6 \phi} \phi_{v}-\left[e^{2 \phi}\left(\mathrm{H} e^{2 \phi}-C\right)+e^{2 \phi}\left(\mathrm{H} e^{2 \phi}+C\right)\right] 2 e^{2 \phi} \phi_{v}=0
\end{aligned}
$$

por:

e as curvaturas principais são dadas por $\bar{\kappa}_{i}(p, q)=\kappa_{i}(u(p), v(q)), i=1,2$. Logo,

$$
\left\{\begin{array}{l}
\bar{E}(p, q)=u^{\prime}(p)^{2} E(u(p), v(q))=\frac{1}{p^{\prime}(u(p))^{2}} E(u(p), v(q)), \\
\bar{F}(p, q)=0 \\
\bar{G}(p, q)=v^{\prime}(q)^{2} G(u(p), v(q))=\frac{1}{q^{\prime}(v(q))^{2}} G(u(p), v(q))
\end{array}\right.
$$

$$
\left\{\begin{aligned}
\bar{E}\left(\bar{\kappa}_{1}-\mathrm{H}\right) & =\frac{1}{p^{\prime}(u(p))^{2}} E\left(\kappa_{1}-\mathrm{H}\right)=|C|, \\
-\bar{G}\left(\bar{\kappa}_{2}-\mathrm{H}\right) & =-\frac{1}{q^{\prime}(v(q))^{2}} G\left(\kappa_{2}-\mathrm{H}\right)=|C|
\end{aligned}\right.
$$




$$
\begin{aligned}
& 2 E G\left(f_{v}-g_{u}\right)+(E g+G e) G_{u}-f\left(E G_{v}-G E_{v}\right) \\
& \quad=-4 \mathrm{H} e^{6 \phi} \phi_{u}+\left[e^{2 \phi}\left(\mathrm{H} e^{2 \phi}+C\right)+e^{2 \phi}\left(\mathrm{H} e^{2 \phi}-C\right)\right] 2 e^{2 \phi} \phi_{u}=0 .
\end{aligned}
$$

Portanto, os coeficientes de I e II também satisfazem as equações de Codazzi-Mainardi, (1.26) e (1.27). Assim, segue do Teorema Fundamental das Superfícies que, a menos de movimentos rígidos de $\mathbb{R}^{3}$, existe uma superfície parametrizada regular $X(u, v)$ com formas quadráticas I e II. Além disso,

$$
\frac{e G-2 f F+g E}{2\left(E G-F^{2}\right)}=\frac{\left(\mathrm{H} e^{2 \phi}+C\right) e^{2 \phi}+\left(\mathrm{H} e^{2 \phi}-C\right) e^{2 \phi}}{2 e^{4 \phi}}=\mathrm{H}
$$

isto é, $X$ possui curvatura média constante igual a $\mathrm{H}$.

Observe que, considerando as formas quadráticas

$$
\begin{aligned}
\overline{\mathrm{I}} & =e^{2 \phi}\left(d u^{2}+d v^{2}\right), \\
\overline{\mathrm{II}} & =\left(\mathrm{H} e^{2 \phi}-C\right) d u^{2}+\left(\mathrm{H} e^{2 \phi}+C\right) d v^{2},
\end{aligned}
$$

com um raciocínio análogo, obtemos, a menos de movimento rígido de $\mathbb{R}^{3}$, uma superfície parametrizada regular $\bar{X}(u, v)$ que possui estas formas quadráticas e de curvatura média constante igual a $\mathrm{H}$.

Observação 2.1.2. Se considerarmos $H \neq 0$ na Proposição 2.1.1, podemos escolher a constante $C$ de tal forma que $C^{2}=\mathrm{H}^{2}$. Neste caso, a equação (2.1) pode ser reescrita como

$$
\Delta \phi=\mathrm{H}^{2}\left(e^{-2 \phi}-e^{2 \phi}\right)=-2 \mathrm{H}^{2} \operatorname{senh}(2 \phi),
$$

ou seja, as superfícies de curvatura média constante $\mathrm{H}$ não nula estão associadas às soluções da equação

$$
\Delta \phi=-4 \mathrm{H}^{2} \operatorname{senh} \phi \cosh \phi .
$$

Considere

$$
\psi(u, v)=\phi\left(\frac{u}{2 \mathrm{H}}, \frac{v}{2 \mathrm{H}}\right) .
$$

Então,

$$
\begin{aligned}
\Delta \psi(u, v) & =\frac{1}{4 \mathrm{H}^{2}} \Delta \phi\left(\frac{u}{2 \mathrm{H}}, \frac{v}{2 \mathrm{H}}\right) \\
& =-\operatorname{senh} \phi\left(\frac{u}{2 \mathrm{H}}, \frac{v}{2 \mathrm{H}}\right) \cosh \phi\left(\frac{u}{2 \mathrm{H}}, \frac{v}{2 \mathrm{H}}\right) \\
& =-\operatorname{senh} \psi(u, v) \cosh \psi(u, v) .
\end{aligned}
$$

Conseqüentemente, as superfícies de curvatura média constante não nula estão associadas às soluções da equação $\Delta \psi=-\operatorname{senh} \psi \cosh \psi$.

Como conseqüência da Proposição 2.1.1 temos o seguinte

Corolário 2.1.3. A cada superfície mínima sem pontos planares podemos associar uma familia a um parâmetro de soluções da equação de Liouville:

$$
\Delta \phi=e^{-2 \phi} .
$$

Reciprocamente, a cada solução de (2.6) podemos associar, a menos de um movimento rígido de $\mathbb{R}^{3}$, uma superfície mínima. 
Demonstração. Seja $X(u, v)$ uma superfície mínima e sem pontos planares. Então $K \neq 0$ e $\mathrm{H}^{2}-K \neq 0$, ou seja, $X$ não possui pontos umbílicos. Assim, pela prova da Proposição 2.1.1, a esta superfície está associada uma família a um parâmetro $\left\{\phi_{C}\right\}_{C \neq 0}$ de soluções da equação

$$
\Delta \phi=C^{2} e^{-2 \phi} \text {. }
$$

Definindo

$$
\bar{\phi}_{C}(u, v)=\phi_{C}\left(\frac{u}{C}, \frac{v}{C}\right)
$$

como

$$
\Delta \bar{\phi}_{C}(u, v)=\frac{1}{C^{2}} \Delta \phi_{C}\left(\frac{u}{C}, \frac{v}{C}\right)=e^{-2 \phi_{C}\left(\frac{u}{C}, \frac{v}{C}\right)}=e^{-2 \bar{\phi}_{C}(u, v)},
$$

obtemos que $\left\{\bar{\phi}_{C}\right\}_{C \neq 0}$ é uma família a um parâmetro de soluções de (2.6).

Por outro lado, para cada solução $\phi$ de $(2.6)$, defina $\bar{\phi}(u, v)=\phi(C u, C v)$, onde $C$ é uma constante não nula. Então,

$$
\Delta \bar{\phi}(u, v)=C^{2} \Delta \phi(C u, C v)=C^{2} e^{-2 \phi(C u, C v)}=C^{2} e^{-2 \bar{\phi}(u, v)} .
$$

Portanto, pela Proposição 2.1.1, a solução $\bar{\phi}$ da equação acima está associada, a menos de movimento rígido de $\mathbb{R}^{3}$, a uma superfície mínima.

Veremos agora duas aplicações do Corolário 2.1.3.

Exemplo 2.1.4 (Superfície de Enneper). Considere a parametrização da superfície de Enneper dada por:

$$
X(u, v)=\left(u-\frac{u^{3}}{3}+u v^{2}, v-\frac{v^{3}}{3}+v u^{2}, u^{2}-v^{2}\right), \quad(u, v) \in \mathbb{R}^{2} .
$$

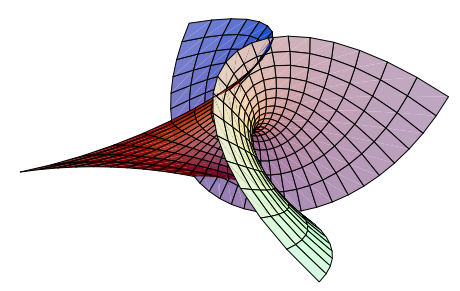

Figura 2.1: Superfície de Enneper.

Os coeficientes da primeira e segunda formas quadráticas de $X$ são dados por (veja Exemplo A.4.4):

$$
\begin{gathered}
E=G=\left(1+u^{2}+v^{2}\right)^{2}, \quad F=0, \\
e=2, \quad g=-2, \quad f=0 .
\end{gathered}
$$

Portanto $X$ é uma superfície mínima e com curvatura gaussiana

$$
K=-\frac{4}{\left(1+u^{2}+v^{2}\right)^{4}}<0,
$$

ou seja, sem pontos planares. Também, como $F=0=f$, as curvas coordenadas de $X$ são linhas de curvatura e, portanto, as curvaturas principais satisfazem as equações (2.2):

$$
\kappa_{1} E=2 \quad \text { e } \quad-\kappa_{2} G=2 .
$$


Para cada constante $C \neq 0$, considere a mudança de parâmetros como em (2.3):

$$
\begin{aligned}
h: \mathbb{R}^{2} & \longrightarrow \mathbb{R}^{2} \\
(u, v) & \longmapsto \sqrt{\frac{|C|}{2}}(u, v) .
\end{aligned}
$$

Então, os coeficientes da primeira e segunda formas quadráticas da superfície $\bar{X}=X \circ h$ são dados por:

$$
\begin{gathered}
\bar{E}=\bar{G}=\frac{|C|}{2}\left(1+\frac{|C|}{2} u^{2}+\frac{|C|}{2} v^{2}\right)^{2}, \quad \bar{F}=0, \\
\bar{e}=|C|, \quad \bar{g}=-|C|, \quad \bar{f}=0 .
\end{gathered}
$$

Considerando a função $\phi(u, v)$ tal que $\bar{E}=\bar{G}=e^{2 \phi}$, isto é,

$$
\phi(u, v)=\log \left(\sqrt{\frac{|C|}{2}}\left(1+\frac{|C|}{2} u^{2}+\frac{|C|}{2} v^{2}\right)\right)
$$

resulta que

$$
\Delta \phi=\frac{2|C|}{\left(1+\frac{|C|}{2} u^{2}+\frac{|C|}{2} v^{2}\right)^{2}}=C^{2} e^{-2 \phi},
$$

ou seja, $\phi$ é solução de (2.7). Portanto, definindo $\bar{\phi}(u, v)=\phi\left(\frac{u}{C}, \frac{v}{C}\right)$, obtemos que

$$
\bar{\phi}(u, v)=\log \left(2|C|+u^{2}+v^{2}\right)-\log (2 \sqrt{2|C|})
$$

representa uma família a um parâmetro de soluções da equação (2.6).

Exemplo 2.1.5. Considere o catenóide

$$
X(u, v)=(\cosh v \cos u, \cosh v \operatorname{sen} u, v), \quad(u, v) \in \mathbb{R}^{2} .
$$

Os coeficientes da primeira e segunda formas quadráticas de $X$ são dados por:

$$
\begin{array}{ll}
E=G=\cosh ^{2} v, \quad F=0, \\
e=-1, \quad g=1, \quad f=0,
\end{array}
$$

de onde vemos que $X$ é uma superfície mínima sem pontos planares, com curvas coordenadas sendo linhas de curvatura. Mais ainda, $\kappa_{1}$ e $\kappa_{2}$ satisfazem as equações (2.2):

$$
-\kappa_{1} E=1 \quad \text { e } \quad \kappa_{2} G=1 .
$$

Para cada constante $C \neq 0$, considere a mudança de parâmetros dada em (2.3):

$$
\begin{aligned}
h: \mathbb{R}^{2} & \longrightarrow \mathbb{R}^{2} \\
(u, v) & \longmapsto \sqrt{|C|}(u, v) .
\end{aligned}
$$

Então, a superfície $\bar{X}(u, v)=(X \circ h)(u, v)$ possui:

$$
\begin{array}{ccc}
\bar{E} & =\bar{G}=|C| \cosh ^{2}(\sqrt{|C|} v), & \bar{F}=0, \\
\bar{e} & =-|C|, \quad \bar{g}=|C|, & f=0,
\end{array}
$$


como coeficientes da primeira e da segunda formas quadráticas. Se $\phi(u, v)$ é uma função diferenciável tal que $\bar{E}=\bar{G}=e^{2 \phi}$, então

$$
e^{2 \phi}=|C| \cosh ^{2}(\sqrt{|C|} v),
$$

isto é,

$$
\phi=\log (\sqrt{|C|} \cosh (\sqrt{|C|} v))
$$

Portanto,

$$
\Delta \phi=\frac{|C|}{\cosh ^{2}(\sqrt{|C|} v)}=C^{2} e^{-2 \phi}
$$

Assim, definindo

$$
\bar{\phi}(u, v)=\phi\left(\frac{u}{C}, \frac{v}{C}\right)=\log \left(\sqrt{|C|} \cosh \left(\frac{v}{\sqrt{|C|}}\right)\right)
$$

obtemos uma família a um parâmetro de soluções de $\Delta \bar{\phi}=e^{-2 \bar{\phi}}$.

\subsection{Superfícies de curvatura gaussiana constante positiva}

Na Observação 2.1.2 vimos que as superfícies de curvatura média constante não nula estão associadas às soluções da equação

$$
\Delta \psi=-\operatorname{senh} \psi \cosh \psi
$$

Provaremos, a seguir, que as superfícies de curvatura gaussiana constante positiva também estão associadas a soluções desta equação, conhecida como equação de Sine-Gordon elíptica. A interpretação geométrica deste fato decorre do Teorema de Bonnet, dado pelo Corolário 3.1.10.

Proposição 2.2.1. A toda superfície de curvatura gaussiana constante $K>0$, sem pontos umbilicos, podemos associar uma solução da equação

$$
\Delta \phi=-\operatorname{senh} \phi \cosh \phi .
$$

Reciprocamente, fixado $K>0$, a cada solução não nula da equação (2.8) podemos associar, a menos de movimentos rígidos de $\mathbb{R}^{3}$, um par de superfícies de curvatura gaussiana constante igual a $K$.

Demonstração. Seja $X: U \subset \mathbb{R}^{2} \rightarrow \mathbb{R}^{3}$ uma superfície parametrizada regular de curvatura gaussiana constante $K>0$ e sem pontos umbílicos. Supondo que $X$ seja uma parametrização principal, temos que as formas quadráticas desta superfície são dadas por:

$$
\begin{aligned}
\mathrm{I} & =E d u^{2}+G d v^{2}, \\
\mathrm{II} & =e d u^{2}+g d v^{2},
\end{aligned}
$$

e as curvaturas principais são $\kappa_{1}=\frac{e}{E}$ e $\kappa_{2}=\frac{g}{G}$. Também, da Proposição 1.3.7, as curvaturas principais satisfazem as equações de Codazzi-Mainardi

$$
\left\{\begin{array}{l}
\frac{1}{\kappa_{1}-\kappa_{2}} \frac{\partial \kappa_{1}}{\partial v}=-\frac{\partial}{\partial v}(\log \sqrt{E}), \\
\frac{1}{\kappa_{2}-\kappa_{1}} \frac{\partial \kappa_{2}}{\partial u}=-\frac{\partial}{\partial u}(\log \sqrt{G}),
\end{array}\right.
$$


que podem ser reescritas como

$$
\left\{\begin{array}{l}
\frac{\kappa_{1}}{\kappa_{1}^{2}-K} \frac{\partial \kappa_{1}}{\partial v}=-\frac{\partial}{\partial v}(\log \sqrt{E}) \\
\frac{\kappa_{2}}{\kappa_{2}^{2}-K} \frac{\partial \kappa_{2}}{\partial u}=-\frac{\partial}{\partial u}(\log \sqrt{G})
\end{array}\right.
$$

pois $\kappa_{1} \kappa_{2}=K$.

Como a superfície $X$ não tem pontos umbílicos e $K>0$ (isto é, $\kappa_{1}, \kappa_{2}$ possuem o mesmo sinal), temos que

$$
\begin{aligned}
& \kappa_{1}^{2}-K=\kappa_{1}\left(\kappa_{1}-\kappa_{2}\right) \neq 0, \\
& \kappa_{2}^{2}-K=-\kappa_{2}\left(\kappa_{1}-\kappa_{2}\right) \neq 0,
\end{aligned}
$$

portanto têm sinais opostos. Supondo que $\kappa_{1}^{2}>K$ e $\kappa_{2}^{2}<K$, as equações (2.9) se reduzem a

$$
\left\{\begin{array}{l}
\frac{\partial}{\partial v}\left(\log \left(E\left(\kappa_{1}^{2}-K\right)\right)\right)=0, \\
\frac{\partial}{\partial u}\left(\log \left(G\left(K-\kappa_{2}^{2}\right)\right)\right)=0 .
\end{array}\right.
$$

Logo, existem funções positivas $\psi_{1}(u)$ e $\psi_{2}(v)$ tais que

$$
\left\{\begin{array}{l}
E\left(\kappa_{1}^{2}-K\right)=\psi_{1}(u), \\
G\left(K-\kappa_{2}^{2}\right)=\psi_{2}(v) .
\end{array}\right.
$$

Então, a menos de uma reparametrização ${ }^{2}$ de $X$, podemos supor que

$$
\left\{\begin{array}{l}
E\left(\kappa_{1}^{2}-K\right)=1, \\
G\left(K-\kappa_{2}^{2}\right)=1
\end{array}\right.
$$

de onde resulta que

$$
G-E=\frac{1}{K-\kappa_{2}^{2}}-\frac{1}{\kappa_{1}^{2}-K}=\frac{1}{K}
$$

\footnotetext{
${ }^{2}$ Como $\psi_{1}$ é função somente de $u$ e $\psi_{2}$ é função somente de $v$, podemos definir duas funções $p$ e $q$ pondo

$$
p(u)=\int \sqrt{\psi_{1}(u)} d u, \quad q(v)=\int \sqrt{\psi_{2}(v)} d v .
$$

Como $p^{\prime}$ e $q^{\prime}$ não se anulam, pelo Teorema da Função Inversa, existem as inversas $u=u(p)$ e $v=v(q)$ de $p$ e $q$, respectivamente. Definindo $\bar{X}(p, q)=X(u(p), v(q))$, os coeficientes da primeira forma quadrática de $\bar{X}$ são dados por:

$$
\left\{\begin{array}{l}
\bar{E}(p, q)=u^{\prime}(p)^{2} E(u(p), v(q))=\frac{1}{p^{\prime}(u(p))^{2}} E(u(p), v(q)), \\
\bar{F}(p, q)=0 \\
\bar{G}(p, q)=v^{\prime}(q)^{2} G(u(p), v(q))=\frac{1}{q^{\prime}(v(q))^{2}} G(u(p), v(q))
\end{array}\right.
$$

e as curvaturas principais são dadas por $\bar{\kappa}_{i}(p, q)=\kappa_{i}(u(p), v(q)), i=1,2$. Logo,

$$
\begin{aligned}
& \bar{E}\left(\bar{\kappa}_{1}^{2}-K\right)=\frac{1}{p^{\prime}(u)^{2}} E\left(\kappa_{1}^{2}-K\right)=1, \\
& \bar{G}\left(K-\bar{\kappa}_{2}^{2}\right)=\frac{1}{q^{\prime}(v)^{2}} G\left(K-\kappa_{2}^{2}\right)=1 .
\end{aligned}
$$


Seja $\phi(u, v)$ uma função diferenciável não nula tal que

$$
E=\frac{1}{K} \operatorname{senh}^{2} \phi \quad \text { e } \quad G=\frac{1}{K} \cosh ^{2} \phi .
$$

Logo, a equação de Gauss

$$
K=-\frac{1}{\sqrt{E G}}\left[\left(\frac{(\sqrt{E})_{v}}{\sqrt{G}}\right)_{v}+\left(\frac{(\sqrt{G})_{u}}{\sqrt{E}}\right)_{u}\right]
$$

se reduz a

$$
K=-\frac{K \Delta \phi}{\operatorname{senh} \phi \cosh \phi}
$$

ou seja, $\phi$ satisfaz a equação (2.8).

No caso em que $\kappa_{1}^{2}<K$ e $\kappa_{2}^{2}>K$ obtemos, de forma análoga, $E-G=\frac{1}{K}$ e, considerando

$$
E=\frac{1}{K} \cosh ^{2} \phi, \quad G=\frac{1}{K} \operatorname{senh}^{2} \phi,
$$

resulta que $\phi$ é solução de (2.8).

Por outro lado, se $\phi$ é solução não nula de (2.8), para cada $K>0$ fixado, considere as formas quadráticas

$$
\begin{aligned}
\mathrm{I} & =\frac{1}{K}\left(\operatorname{senh}^{2} \phi d u^{2}+\cosh ^{2} \phi d v^{2}\right), \\
\mathrm{II} & =\frac{1}{\sqrt{K}} \operatorname{senh} \phi \cosh \phi\left(d u^{2}+d v^{2}\right) .
\end{aligned}
$$

Note que

$$
\frac{e g-f^{2}}{E G-F^{2}}=K
$$

e, como $\phi$ é solução de (2.8), temos que

$$
-\frac{1}{\sqrt{E G}}\left[\left(\frac{(\sqrt{E})_{v}}{\sqrt{G}}\right)_{v}+\left(\frac{(\sqrt{G})_{u}}{\sqrt{E}}\right)_{u}\right]=-\frac{K}{\operatorname{senh} \phi \cosh \phi} \Delta \phi=K
$$

isto é, vale a equação de Gauss. Da mesma maneira vemos que

$$
\begin{aligned}
& 2 E G\left(e_{v}-f_{u}\right)-(E g+G e) E_{v}-f\left(E G_{u}-G E_{u}\right) \\
& =2 \frac{1}{K^{2}} \operatorname{senh}^{2} \phi \cosh ^{2} \phi \frac{1}{\sqrt{K}}\left(\cosh ^{2} \phi+\operatorname{senh}^{2} \phi\right) \phi_{v} \\
& \quad-\frac{1}{K^{\frac{3}{2}}} \operatorname{senh} \phi \cosh \phi\left(\operatorname{senh}^{2} \phi+\cosh ^{2} \phi\right) 2 \frac{1}{K} \cosh \phi \operatorname{senh} \phi \phi_{v}=0, \\
& 2 E G\left(f_{v}-g_{u}\right)+(E g+G e) G_{u}-f\left(E G_{v}-G E_{v}\right) \\
& =-2 \frac{1}{K^{2}} \operatorname{senh}^{2} \phi \cosh ^{2} \phi \frac{1}{\sqrt{K}}\left(\cosh ^{2} \phi+\operatorname{senh}^{2} \phi\right) \phi_{u} \\
& \quad+\frac{1}{K^{\frac{3}{2}}} \operatorname{senh} \phi \cosh \phi\left(\cosh ^{2} \phi+\operatorname{senh}^{2} \phi\right) 2 \frac{1}{K} \cosh \phi \operatorname{senh} \phi \phi_{u}=0,
\end{aligned}
$$

ou seja, os coeficientes das formas quadráticas também satisfazem as equações de CodazziMainardi. Logo, do Teorema Fundamental das Superfícies, a menos de um movimento rígido de 
$\mathbb{R}^{3}$, existe uma superfície $X(u, v)$ de curvatura gaussiana igual a $K$ e com formas quadráticas dadas por I e II.

Da mesma forma, se considerarmos as formas

$$
\begin{aligned}
\overline{\mathrm{I}} & =\frac{1}{K}\left(\cosh ^{2} \phi d u^{2}+\operatorname{senh}^{2} \phi d v^{2}\right), \\
\overline{\mathrm{II}} & =\frac{1}{\sqrt{K}} \operatorname{senh} \phi \cosh \phi\left(d u^{2}+d v^{2}\right),
\end{aligned}
$$

obtemos uma superfície $\bar{X}(u, v)$ de curvatura gaussiana constante $K$ e com formas quadráticas $\overline{\mathrm{I}}$ e II.

Note que as superfícies de curvatura gaussiana positiva não admitem direções assintóticas mas, em cada ponto não umbílico, existe um único par de direções conjugadas simétricas em relação às direções principais, ou seja, existem direções características (veja página 141). Assim, segue uma interpretação geométrica das soluções da equação (2.8).

Proposição 2.2.2. Seja $\phi$ uma solução de equação (2.8) e considere $X(u, v)$ e $\bar{X}(u, v)$ o par de superfícies de curvatura gaussiana $K$ constante positiva associadas a esta solução. Defina $\psi(u, v)$ por

$$
\cos \psi=\frac{\operatorname{senh} \phi}{\sqrt{\cosh 2 \phi}}, \quad \operatorname{sen} \psi=\frac{\cosh \phi}{\sqrt{\cosh 2 \phi}}
$$

$e \bar{\psi}=\frac{\pi}{2}-\psi$. Então o ângulo formado pelas direções características da superfície $X$ (respectivamente $\bar{X}$ ) é $2 \psi$ (respectivamente $2 \bar{\psi}$ ).

Demonstração. Da demonstração da Proposição 2.2.1, as formas quadráticas de $X$ são dadas por

$$
\begin{aligned}
\mathrm{I} & =\frac{1}{K}\left(\operatorname{senh}^{2} \phi d u^{2}+\cosh ^{2} \phi d v^{2}\right), \\
\mathrm{II} & =\frac{1}{\sqrt{K}} \operatorname{senh} \phi \cosh \phi\left(d u^{2}+d v^{2}\right) .
\end{aligned}
$$

Observe primeiramente que, como $f=0=F$, então $X_{u}$ e $X_{v}$ são os campos de direções principais (Proposição A.3.6). Considere, agora, os campos de vetores definidos por

$$
w_{1}=X_{u}+X_{v}, \quad w_{2}=X_{u}-X_{v} .
$$

Então $\left\|w_{1}\right\|=\left\|w_{2}\right\|$ e também

$$
\mathrm{II}\left(w_{1}, w_{2}\right)=e-g=0,
$$

isto é, $w_{1}$ e $w_{2}$ são campos conjugados. Portanto, como

$$
\frac{\left\langle w_{1}, X_{u}\right\rangle}{\left\|w_{1}\right\|\left\|X_{u}\right\|}=\frac{\left\|X_{u}\right\|}{\left\|w_{1}\right\|}=\frac{\left\|X_{u}\right\|}{\left\|w_{2}\right\|}=\frac{\left\langle w_{2}, X_{u}\right\rangle}{\left\|w_{2}\right\|\left\|X_{u}\right\|}
$$

e $F=0, w_{1}$ e $w_{2}$ são simétricos em relação às direções principais. Logo, $w_{1}$ e $w_{2}$ são direções características de $X$ que formam um ângulo igual a $2 \psi$ pois:

$$
\frac{\left\langle w_{1}, w_{2}\right\rangle}{\left\|w_{1}\right\|\left\|w_{2}\right\|}=\frac{\operatorname{senh}^{2} \phi-\cosh ^{2} \phi}{\cosh (2 \phi)}=\cos ^{2} \psi-\operatorname{sen}^{2} \psi=\cos (2 \psi)
$$


Da mesma maneira, vimos que a superfície $\bar{X}$ é determinada pelas formas quadráticas

$$
\begin{aligned}
\overline{\mathrm{I}} & =\frac{1}{K}\left(\cosh ^{2} \phi d u^{2}+\operatorname{senh}^{2} \phi d v^{2}\right), \\
\overline{\mathrm{II}} & =\frac{1}{\sqrt{K}} \operatorname{senh} \phi \cosh \phi\left(d u^{2}+d v^{2}\right) .
\end{aligned}
$$

Neste caso o ângulo formado pelas direções características de $\bar{X}$ :

$$
\bar{w}_{1}=\bar{X}_{u}+\bar{X}_{v} \quad \text { e } \quad \bar{w}_{2}=\bar{X}_{u}-\bar{X}_{v},
$$

é $2 \bar{\psi}$ pois

$$
\frac{\left\langle\bar{w}_{1}, \bar{w}_{2}\right\rangle}{\left\|\bar{w}_{1}\right\|\left\|\bar{w}_{2}\right\|}=-\cos (2 \psi)=-\cos (\pi-2 \bar{\psi})=\cos (2 \bar{\psi})
$$

\subsection{Superfícies de curvatura gaussiana constante negativa}

O estudo das superfícies pseudoesféricas, isto é, superfícies com curvatura gaussiana constante negativa, está relacionado ao estudo das soluções da equação diferencial às derivadas parciais denominada equação de Sine-Gordon. Existem duas versões desta equação. A primeira é ligada à procura de parametrizações principais com curvatura gaussiana constante negativa e é dada por:

$$
\phi_{u u}-\phi_{v v}=\operatorname{sen} \phi \cos \phi .
$$

Considerando a mudança de parâmetros

$$
\left\{\begin{array}{l}
u=x+y, \\
v=x-y
\end{array}\right.
$$

se $\psi(x, y)=2 \phi(u(x, y), v(x, y))$, então obtemos a segunda forma da equação de Sine-Gordon:

$$
\begin{aligned}
\psi_{x y} & =2 \phi_{x y}=2\left(\phi_{u u}-\phi_{v v}\right) \\
& =2 \operatorname{sen} \phi \cos \phi=\operatorname{sen} \psi
\end{aligned}
$$

Esta forma está associada à procura de parametrizações assintóticas de curvatura gaussiana constante negativa, que veremos na página 27.

\section{Parametrizações principais de curvatura gaussiana constante negativa}

Inicialmente veremos dois resultados que serão úteis para mostrar a relação entre as parametrizações principais de curvatura gaussiana constante negativa e as soluções da equação de SineGordon.

Lema 2.3.1. Seja $X: U \subset \mathbb{R}^{2} \rightarrow \mathbb{R}^{3}$ uma parametrização principal de curvatura gaussiana constante $K<0$ e com métrica $\mathrm{I}=E d u^{2}+G d v^{2}$. Sendo $\kappa_{1}$ e $\kappa_{2}$ as curvaturas principais de $X$, então existem funções positivas $\psi_{1}(u)$ e $\psi_{2}(v)$ tais que

$$
\left\{\begin{array}{l}
E\left(\kappa_{1}^{2}-K\right)=\psi_{1}(u), \\
G\left(\kappa_{2}^{2}-K\right)=\psi_{2}(v),
\end{array}\right.
$$


ou seja,

$$
\left\{\begin{array}{l}
\kappa_{1}^{2}=K+\frac{\psi_{1}(u)}{E}, \\
\kappa_{2}^{2}=K+\frac{\psi_{2}(v)}{G} .
\end{array}\right.
$$

Demonstração. Sendo $X$ uma parametrização principal sem pontos umbílicos, da Proposição 1.3.7 segue que as curvaturas principais $\kappa_{1}$ e $\kappa_{2}$ satisfazem as equações de Codazzi-Mainardi:

$$
\left\{\begin{array}{l}
\frac{\kappa_{1}}{\kappa_{1}^{2}-K} \frac{\partial \kappa_{1}}{\partial v}=-\frac{\partial}{\partial v}(\log \sqrt{E}) \\
\frac{\kappa_{2}}{\kappa_{2}^{2}-K} \frac{\partial \kappa_{2}}{\partial u}=-\frac{\partial}{\partial u}(\log \sqrt{G})
\end{array}\right.
$$

isto é,

$$
\left\{\begin{array}{l}
\frac{\partial}{\partial v}\left(\log \left(E\left(\kappa_{1}^{2}-K\right)\right)\right)=0 \\
\frac{\partial}{\partial u}\left(\log \left(G\left(\kappa_{2}^{2}-K\right)\right)\right)=0 .
\end{array}\right.
$$

Logo, integrando, existem funções positivas $\psi_{1}(u)$ e $\psi_{2}(v)$ tais que

$$
\left\{\begin{array}{l}
E\left(\kappa_{1}^{2}-K\right)=\psi_{1}(u), \\
G\left(\kappa_{2}^{2}-K\right)=\psi_{2}(v),
\end{array}\right.
$$

o que equivale ao sistema (2.12).

Proposição 2.3.2. Considere $\bar{X}: U \subset \mathbb{R}^{2} \rightarrow \mathbb{R}^{3}$ uma parametrização principal com métrica $\mathrm{I}=\bar{E} d p^{2}+\bar{G} d q^{2}$ e de curvatura gaussiana constante $K<0$. Então existe uma parametrização principal $X$ da forma

$$
X(u, v)=\bar{X}(p(u), q(v))
$$

que reparametriza $\bar{X}$ e cujas curvaturas principais satisfazem

$$
\left\{\begin{array}{l}
E\left(\kappa_{1}^{2}-K\right)=1 \\
G\left(\kappa_{2}^{2}-K\right)=1
\end{array}\right.
$$

Demonstração. Suponha que $X$ seja uma reparametrização positiva de $\bar{X}$ da forma (2.13). Temos, então

$$
X_{u}=p^{\prime}(u) \bar{X}_{p}, \quad X_{v}=q^{\prime}(v) \bar{X}_{q}
$$

e

$$
X_{u u}=p^{\prime \prime}(u) \bar{X}_{p}+p^{\prime}(u)^{2} \bar{X}_{p p}, \quad X_{u v}=p^{\prime}(u) q^{\prime}(v) \bar{X}_{p q}, \quad X_{v v}=q^{\prime \prime}(v) \bar{X}_{q}+q^{\prime}(v)^{2} \bar{X}_{q q} .
$$

Calculando os coeficientes da primeira forma quadrática de $X$, em função dos correspondentes coeficientes de $\bar{X}$, temos

$$
\left\{\begin{array}{l}
E(u, v)=p^{\prime}(u)^{2} \bar{E}(p(u), q(v)), \\
F(u, v)=0 \\
G(u, v)=q^{\prime}(v)^{2} \bar{G}(p(u), q(v)) .
\end{array}\right.
$$


Da mesma maneira, os coeficientes da segunda forma quadrática de $X$, em relação aos de $\bar{X}$, são dados por:

$$
\left\{\begin{array}{l}
e(u, v)=p^{\prime}(u)^{2} \bar{e}(p(u), q(v)), \\
f(u, v)=p^{\prime}(u) q^{\prime}(v) \bar{f}(p(u), q(v))=0, \\
g(u, v)=q^{\prime}(v)^{2} \bar{g}(p(u), q(v)) .
\end{array}\right.
$$

Sendo $\bar{\kappa}_{1}$ e $\bar{\kappa}_{2}$ as curvaturas principais de $\bar{X}$, então (2.15) e (2.16) implicam que

$$
\kappa_{i}(u, v)=\bar{\kappa}_{i}(p(u), q(v)), \quad i=1,2 .
$$

Portanto, do Lema 2.3.1 e usando (2.15), obtemos

$$
\left\{\begin{array}{l}
\kappa_{1}(u, v)^{2}=\bar{\kappa}_{1}(p(u), q(v))^{2}=K+\frac{\psi_{1}(p(u))}{\bar{E}(p(u), q(v))}=K+\frac{p^{\prime}(u)^{2} \psi_{1}(p(u))}{E(u, v)} \\
\kappa_{2}(u, v)^{2}=\bar{\kappa}_{2}(p(u), q(v))^{2}=K+\frac{\psi_{2}(q(v))}{\bar{G}(p(u), q(v))}=K+\frac{q^{\prime}(v)^{2} \psi_{2}(q(v))}{G(u, v)}
\end{array}\right.
$$

ou seja,

$$
\left\{\begin{array}{l}
E\left(\kappa_{1}^{2}-K\right)=p^{\prime}(u)^{2} \psi_{1}(p(u)), \\
G\left(\kappa_{2}^{2}-K\right)=q^{\prime}(v)^{2} \psi_{2}(q(v)) .
\end{array}\right.
$$

Para a equivalência de (2.14) e (2.18), basta escolher $p$ e $q$ tais que

$$
p^{\prime}(u)^{2} \psi_{1}(p(u))=q^{\prime}(v)^{2} \psi_{2}(q(v))=1
$$

Para isto, tome (por exemplo) $p(u)$ e $q(v)$ como sendo as funções inversas de $u(p)$ e $v(q)$, respectivamente, dadas por:

$$
\left\{\begin{array}{l}
u(p)=\int \sqrt{\psi_{1}(p)} d p \\
v(q)=\int \sqrt{\psi_{2}(q)} d q
\end{array}\right.
$$

Proposição 2.3.3. A cada superfície de curvatura gaussiana constante $K<0$, podemos associar uma solução de

$$
\phi_{u u}-\phi_{v v}=\operatorname{sen} \phi \cos \phi .
$$

Reciprocamente, fixado $K<0$, a cada solução não nula da equação (2.19) podemos associar, a menos de um movimento rígido de $\mathbb{R}^{3}$, uma superfície de curvatura gaussiana constante igual a $K$.

Demonstração. Seja $X$ uma superfície de curvatura gaussiana constante $K<0$. Como $X$ não possui pontos umbílicos (pelas Proposições A.5.3 e A.5.4), podemos supor que suas formas quadráticas sejam dadas por:

$$
\begin{aligned}
\mathrm{I} & =E d u^{2}+G d v^{2}, \\
\mathrm{II} & =e d u^{2}+g d v^{2} .
\end{aligned}
$$


Além disso, da Proposição 2.3.2 podemos considerar que as curvaturas principais $\kappa_{1}$ e $\kappa_{2}$ satisfazem

$$
\left\{\begin{array}{l}
E\left(\kappa_{1}^{2}-K\right)=1, \\
G\left(\kappa_{2}^{2}-K\right)=1
\end{array}\right.
$$

e, portanto,

$$
E+G=-\frac{1}{K}
$$

Considerando $\phi(u, v)$ uma função diferenciável não nula tal que

$$
E=-\frac{1}{K} \cos ^{2} \phi \quad \text { e } \quad G=-\frac{1}{K} \operatorname{sen}^{2} \phi,
$$

segue da equação de Gauss (1.25) que

$$
K=-\frac{1}{\sqrt{E G}}\left[\left(\frac{(\sqrt{E})_{v}}{\sqrt{G}}\right)_{v}+\left(\frac{(\sqrt{G})_{u}}{\sqrt{E}}\right)_{u}\right]=-\frac{-K}{\operatorname{sen} \phi \cos \phi}\left(-\phi_{v v}+\phi_{u u}\right),
$$

isto é,

$$
\phi_{u u}-\phi_{v v}=\operatorname{sen} \phi \cos \phi
$$

ou seja, $\phi$ é solução da equação (2.19).

Reciprocamente, para cada $K<0$ fixo, se $\phi$ é solução não nula de (2.19), considere as formas quadráticas dadas por:

$$
\begin{aligned}
\mathrm{I} & =-\frac{1}{K}\left(\cos ^{2} \phi d u^{2}+\operatorname{sen}^{2} \phi d v^{2}\right), \\
\mathrm{II} & =\frac{1}{\sqrt{-K}} \operatorname{sen} \phi \cos \phi\left(d u^{2}-d v^{2}\right) .
\end{aligned}
$$

Note que

$$
\frac{e g-f^{2}}{E G-F^{2}}=K
$$

e como $\phi$ é solução de (2.19), temos que

$$
-\frac{1}{\sqrt{E G}}\left[\left(\frac{(\sqrt{E})_{v}}{\sqrt{G}}\right)_{v}+\left(\frac{(\sqrt{G})_{u}}{\sqrt{E}}\right)_{u}\right]=-\frac{-K}{\operatorname{sen} \phi \cos \phi}\left(\phi_{u u}-\phi_{v v}\right)=K,
$$

isto é, os coeficientes de I satisfazem a equação de Gauss. Da mesma maneira vemos que

$$
\begin{aligned}
& 2 E G\left(e_{v}-f_{u}\right)-(E g+G e) E_{v}-f\left(E G_{u}-G E_{u}\right) \\
& =2 \frac{1}{K^{2}} \operatorname{sen}^{2} \phi \cos ^{2} \phi \frac{1}{\sqrt{-K}}\left(\cos ^{2} \phi-\operatorname{sen}^{2} \phi\right) \phi_{v} \\
& \quad-\frac{1}{-K} \frac{1}{\sqrt{-K}} \operatorname{sen} \phi \cos \phi\left(\operatorname{sen}^{2} \phi-\cos ^{2} \phi\right) 2 \frac{1}{K} \cos \phi \operatorname{sen} \phi \phi_{v}=0, \\
& 2 E G\left(f_{v}-g_{u}\right)+(E g+G e) G_{u}-f\left(E G_{v}-G E_{v}\right) \\
& =-2 \frac{1}{K^{2}} \operatorname{sen}^{2} \phi \cos ^{2} \phi \frac{1}{\sqrt{-K}}\left(\cos ^{2} \phi-\operatorname{sen}^{2} \phi\right) \phi_{u} \\
& \quad+\frac{1}{-K} \frac{1}{\sqrt{-K}} \operatorname{sen} \phi \cos \phi\left(-\cos ^{2} \phi+\operatorname{sen}^{2} \phi\right) 2 \frac{1}{K} \cos \phi \operatorname{sen} \phi \phi_{u}=0,
\end{aligned}
$$

ou seja, os coeficientes das formas quadráticas também satisfazem as equações de CodazziMainardi. Logo, do Teorema Fundamental das Superfícies, a menos de movimento rígido de $\mathbb{R}^{3}$, existe uma superfície $X(u, v)$ de curvatura gaussiana igual a $K<0$ e com formas quadráticas iguais a I e II. 


\section{Parametrizações assintóticas de curvatura gaussiana constante negativa}

Se $X(u, v)$ é a parametrização principal de uma superfície de curvatura gaussiana constante $K<0$ e com formas quadráticas

$$
\begin{aligned}
\mathrm{I} & =-\frac{1}{K}\left(\cos ^{2} \phi d u^{2}+\operatorname{sen}^{2} \phi d v^{2}\right), \\
\mathrm{II} & =\frac{1}{\sqrt{-K}} \operatorname{sen} \phi \cos \phi\left(d u^{2}-d v^{2}\right),
\end{aligned}
$$

considerando as mudanças de parâmetros

$$
\left\{\begin{array}{l}
u=x+y \\
v=x-y
\end{array}\right.
$$

obtemos que $\bar{X}(x, y):=X(u(x, y), v(x, y))$ é uma reparametrização negativa e assintótica de $X$. De fato, considerando os campos tangentes às curvas coordenadas de $\bar{X}$, temos que

$$
\begin{aligned}
& \mathrm{II}\left(\bar{X}_{x}\right)=\operatorname{II}\left(X_{u}+X_{v}\right)=e+g=0, \\
& \mathrm{II}\left(\bar{X}_{y}\right)=\operatorname{II}\left(X_{u}-X_{v}\right)=e+g=0,
\end{aligned}
$$

isto é, tais campos são direções assintóticas.

Além disso, se $\psi(x, y)=2 \phi(u(x, y), v(x, y))$, os coeficientes da primeira forma quadrática de $\bar{X}$ são dados por:

$$
\left\{\begin{array}{l}
\bar{E}(x, y)=-\frac{1}{K}, \\
\bar{F}(x, y)=-\frac{\cos \psi}{K}, \\
\bar{G}(x, y)=-\frac{1}{K} .
\end{array}\right.
$$

Como $\bar{X}$ é uma reparametrização negativa de $X$, temos que sua aplicação normal de Gauss é $\bar{N}(x, y)=-N(u(x, y), v(x, y))$. Assim, os coeficientes da segunda forma quadrática de $\bar{X}$ são:

$$
\left\{\begin{array}{l}
\bar{e}(x, y)=-e-g=0 \\
\bar{f}(x, y)=-e+g=-\frac{\operatorname{sen} \psi}{\sqrt{-K}} \\
\bar{g}(x, y)=-e-g=0
\end{array}\right.
$$

ou seja,

$$
\begin{aligned}
\overline{\mathrm{I}} & =-\frac{1}{K}\left(d x^{2}+2 \cos \psi d x d y+d y^{2}\right), \\
\overline{\mathrm{II}} & =-2 \frac{\operatorname{sen} \psi}{\sqrt{-K}} d x d y .
\end{aligned}
$$

A seguir daremos uma interpretação geométrica das soluções da equação (2.19) em relação às superfícies associadas.

Proposição 2.3.4. Sejam $K<0$ e $X(u, v)$ a superfície de curvatura gaussiana constante $K$ associada a uma solução $\phi$ da equação (2.19). Então, o ângulo formado pelas direções assintóticas desta superfície é $2 \phi(u, v)$. 
Demonstração. Da demonstração da Proposição 2.3.3, temos que $X$ é determinada pelas formas quadráticas:

$$
\begin{aligned}
\mathrm{I} & =-\frac{1}{K}\left(\cos ^{2} \phi d u^{2}+\operatorname{sen}^{2} \phi d v^{2}\right), \\
\mathrm{II} & =\frac{1}{\sqrt{-K}} \operatorname{sen} \phi \cos \phi\left(d u^{2}-d v^{2}\right) .
\end{aligned}
$$

Como vimos acima, considerando os campos de vetores definidos por

$$
w_{1}=X_{u}+X_{v} \quad \text { e } \quad w_{2}=X_{u}-X_{v}
$$

temos $\operatorname{II}\left(w_{1}\right)=\operatorname{II}\left(w_{2}\right)=0$ e, portanto, $w_{1}$ e $w_{2}$ são direções assintóticas de $X$ (veja Proposição A.5.6). Mais ainda, como

$$
\left\|w_{1}\right\|=\left\|w_{2}\right\|=\frac{1}{\sqrt{-K}}
$$

$\mathrm{e}$

$$
\left\langle w_{1}, w_{2}\right\rangle=E-G=\frac{1}{\sqrt{-K}}\left(\cos ^{2} \phi-\operatorname{sen}^{2} \phi\right)=\frac{1}{\sqrt{-K}} \cos (2 \phi),
$$

obtemos que

$$
\frac{\left\langle w_{1}, w_{2}\right\rangle}{\left\|w_{1}\right\|\left\|w_{2}\right\|}=\cos (2 \phi)
$$

de onde concluímos que o ângulo formado por $w_{1}$ e $w_{2}$ é $2 \phi$.

Veremos, a seguir, uma aplicação da Proposição 2.3.3. Mas antes apresentaremos um resultado que será útil nesta aplicação.

Lema 2.3.5. Seja $a=\log \tan \left(\frac{\psi}{2}\right)$. Então

$$
\operatorname{sen} \psi=\operatorname{sech} a, \quad \cos \psi=-\tanh a .
$$

Demonstração. De fato,

$$
\operatorname{sech} a=\frac{2}{e^{a}+e^{-a}}=\frac{2}{\tan \left(\frac{\psi}{2}\right)+\cot \left(\frac{\psi}{2}\right)}=2 \operatorname{sen}\left(\frac{\psi}{2}\right) \cos \left(\frac{\psi}{2}\right)=\operatorname{sen} \psi
$$

$\mathrm{e}$

$$
\tanh a=\frac{e^{a}-e^{-a}}{e^{a}+e^{-a}}=\frac{\tan \left(\frac{\psi}{2}\right)-\cot \left(\frac{\psi}{2}\right)}{\tan \left(\frac{\psi}{2}\right)+\cot \left(\frac{\psi}{2}\right)}=\operatorname{sen}^{2}\left(\frac{\psi}{2}\right)-\cos ^{2}\left(\frac{\psi}{2}\right)=-\cos \psi
$$

Exemplo 2.3.6. Considere a pseudo-esfera parametrizada por:

$$
\bar{X}(p, q)=\left(\operatorname{sen} p \cos q, \operatorname{sen} p \operatorname{sen} q, \cos p+\log \tan \left(\frac{p}{2}\right)\right) .
$$

Os coeficientes da primeira e da segunda forma quadrática são dados por:

$$
\begin{aligned}
\bar{E}=\cot ^{2} p, & \bar{F}=0, & & \bar{G}=\operatorname{sen}^{2} p ; \\
\bar{e}=-\cot p, & \bar{f}=0, & & \bar{g}=\operatorname{sen} p \cos p .
\end{aligned}
$$


Segue então que $\bar{X}$ é uma parametrização principal e

$$
\bar{\kappa}_{1}=\frac{\bar{e}}{\bar{E}}=-\tan p, \quad \bar{\kappa}_{2}=\frac{\bar{g}}{\bar{G}}=\cot p,
$$

ou seja, $K=-1$. Assim, da Proposição 2.3.2 temos que existe uma reparametrização $X$ da forma

$$
X(u, v)=\bar{X}(p(u), q(v))
$$

e tal que os coeficientes da primeira forma quadrática de $X$ e suas curvaturas principais satisfazem

$$
\left\{\begin{array}{l}
E\left(\kappa_{1}^{2}+1\right)=1 \\
G\left(\kappa_{2}^{2}+1\right)=1
\end{array}\right.
$$

Observe que

$$
\left\{\begin{array}{l}
E(u, v)=p^{\prime}(u)^{2} \bar{E}(p(u), q(v))=p^{\prime}(u)^{2} \cot ^{2} p(u), \\
F(u, v)=0 \\
G(u, v)=q^{\prime}(v)^{2} \bar{G}(p(u), q(v))=q^{\prime}(v)^{2} \operatorname{sen}^{2} p(u)
\end{array}\right.
$$

e

$$
\left\{\begin{array}{l}
\kappa_{1}(u, v)=\bar{\kappa}_{1}(p(u), q(v))=-\tan p(u), \\
\kappa_{2}(u, v)=\bar{\kappa}_{2}(p(u), q(v))=\cot p(u) .
\end{array}\right.
$$

Substituindo (2.22) e (2.23) na primeira equação de (2.21), temos

$$
p^{\prime}(u)^{2} \cot ^{2} p(u)\left[\tan ^{2} p(u)+1\right]=1,
$$

isto é,

$$
p^{\prime}(u)=\gamma \operatorname{sen} p(u), \quad \gamma= \pm 1
$$

Integrando, temos

$$
p(u)=2 \arctan \left(\delta e^{\gamma u+c}\right),
$$

onde $\delta= \pm 1$ e $c$ é a constante de integração. Da mesma maneira, substituindo (2.22) e (2.23) na segunda equação de (2.21), temos

$$
q^{\prime}(v)^{2} \operatorname{sen}^{2} p(u)\left[\cot ^{2} p(u)+1\right]=1,
$$

isto é,

$$
q^{\prime}(v)=\gamma
$$

ou seja,

$$
q(v)=\gamma v+d, \quad d \in \mathbb{R} .
$$

Para $c=d=0$ e $\gamma=\delta=1$, temos que

$$
\left\{\begin{array}{l}
p(u)=2 \arctan \left(e^{u}\right), \\
q(v)=v
\end{array}\right.
$$

e, portanto,

$$
X(u, v)=\left(\operatorname{sen} p(u) \cos v, \operatorname{sen} p(u) \operatorname{sen} v, \cos p(u)+\log \tan \left(\frac{p(u)}{2}\right)\right) .
$$


Segue do Lema 2.3.1 que, como $u=\log \tan \left(\frac{p(u)}{2}\right)$, então

$$
\operatorname{sen} p(u)=\operatorname{sech} u, \quad \cos p(u)=-\tanh u
$$

e, portanto,

$$
X(u, v)=(\operatorname{sech} u \cos v, \operatorname{sech} u \operatorname{sen} v, u-\tanh u) .
$$

Logo, de (2.22) temos que

$$
\left\{\begin{array}{l}
E(u, v)=\operatorname{sen}^{2} p(u) \cot ^{2} p(u)=\tanh ^{2} u \\
F(u, v)=0 \\
G(u, v)=\operatorname{sen}^{2} p(u)=\operatorname{sech}^{2} u
\end{array}\right.
$$

e de (2.23)

$$
\left\{\begin{array}{l}
\kappa_{1}(u, v)=\operatorname{cossech} u \\
\kappa_{2}(u, v)=-\operatorname{senh} u
\end{array}\right.
$$

Note que

$$
E+G=\tanh ^{2} u+\operatorname{sech}^{2} u=1
$$

e, portanto, definindo $\phi(u)$ por

$$
\cos \phi=\tanh u \quad \text { e } \quad \operatorname{sen} \phi=\operatorname{sech} u,
$$

segue da Proposição 2.3.3 que $\phi$ é solução da equação de Sine-Gordon (2.19). 


\section{Capítulo 3}

\section{Congruências de retas pseudo-esféricas}

O presente capítulo é dedicado ao estudo de congruências de retas no espaço Euclidiano $\mathbb{R}^{3}$ e das superfícies que são relacionadas por tais tipos de congruências.

Começaremos estudando o conceito de paralelismo entre duas superfícies, provando um resultado (Proposição 3.1.7) que nos fornece as relações entre as curvaturas média e gaussiana de superfícies paralelas. Em seguida, daremos algumas interessantes conseqüências deste resultado, entre elas, o Teorema de Bonnet (Corolário 3.1.10): "Superfícies de curvatura média constante não nula são paralelas a superfícies de curvatura gaussiana constante positiva".

\subsection{Congruência de retas}

Definição 3.1.1. Chama-se congruência de retas uma família a dois parâmetros de retas de $\mathbb{R}^{3}$.

Definição 3.1.2. Seja dada uma superfície parametrizada regular $X(u, v),(u, v) \in U \subset \mathbb{R}^{2}$.

(i) Se $e(u, v),(u, v) \in U$, for um campo de vetores unitários tangentes a esta superfície, então a congruência

$$
X(u, v)+\lambda e(u, v), \quad \lambda \in I \subset \mathbb{R},
$$

é denominada congruência de retas tangentes à superfície $X$.

(ii) Se $N(u, v)$ for um campo de vetores unitário e normal a $X(u, v)$, então a congruência

$$
X(u, v)+\lambda N(u, v), \quad \lambda \in I \subset \mathbb{R},
$$

é denominada congruência de retas normais a $X$.

Definição 3.1.3. Considere duas superfícies parametrizadas regulares $X, \bar{X}: U \subset \mathbb{R}^{2} \rightarrow \mathbb{R}^{3}$, com traços dados, respectivamente, por $S=X(U)$ e $\bar{S}=\bar{X}(U)$ e um difeomorfismo $l: S \rightarrow \bar{S}$ tal que, para todo $p \in S$, resulte $l(p) \neq p$. Então $l$ determina uma congruência de retas em $\mathbb{R}^{3}$, consistindo na família

$$
p+\lambda \frac{p-l(p)}{\|p-l(p)\|}, \quad \lambda \in I \subset \mathbb{R},
$$

denominada congruência de retas entre as superfícies $S$ e $\bar{S}$. 
Definição 3.1.4. Sejam $X: U \subset \mathbb{R}^{2} \rightarrow \mathbb{R}^{3}$ uma superfície parametrizada regular e $\kappa_{1}, \kappa_{2}$ suas curvaturas principais. As parametrizações focais associadas a $X$ são definidas por:

$$
\begin{aligned}
& Y_{1}(u, v)=X(u, v)+\frac{1}{\kappa_{1}(u, v)} e_{3}(u, v), \\
& Y_{2}(u, v)=X(u, v)+\frac{1}{\kappa_{2}(u, v)} e_{3}(u, v),
\end{aligned}
$$

onde $e_{3}$ é um campo de vetores unitário e normal a $X$.

Proposição 3.1.5. Seja $X: U \subset \mathbb{R}^{2} \rightarrow \mathbb{R}^{3}$ uma superfície parametrizada regular sem pontos umbilicos e com curvatura gaussiana $K$ não nula. Se $d \kappa_{1} \wedge \omega_{2}$ e $d \kappa_{2} \wedge \omega_{1}$ nunca se anulam, então $Y_{1}$ e $Y_{2}$ dadas por (3.1) são superfícies parametrizadas regulares.

Demonstração. Seja $\left\{e_{1}, e_{2}, e_{3}\right\}$ um triedro móvel principal associado a $X$. Denotando por $\omega_{1}, \omega_{2}, \omega_{i j}, i, j=1,2,3$, as 1 -formas diferenciais associadas ao triedro móvel de $X$, da diferencial de $Y_{1}$ temos

$$
\begin{aligned}
d Y_{1} & =d X-\frac{d \kappa_{1}}{\kappa_{1}^{2}} e_{3}+\frac{1}{\kappa_{1}} d e_{3} \\
& =\left(\omega_{1}-\frac{1}{\kappa_{1}} \omega_{13}\right) e_{1}+\left(\omega_{2}-\frac{1}{\kappa_{1}} \omega_{23}\right) e_{2}-\frac{d \kappa_{1}}{\kappa_{1}^{2}} e_{3},
\end{aligned}
$$

onde usamos, na segunda igualdade, as relações (1.3), (1.4) e (1.5). Como $e_{1}, e_{2}$ são campos de direções principais e a superfície $X$ não possui pontos umbílicos, segue da Proposição 1.3.6 que $\omega_{13}=\kappa_{1} \omega_{1}$ e $\omega_{23}=\kappa_{2} \omega_{2}$. Assim,

$$
d Y_{1}=\left(1-\frac{\kappa_{2}}{\kappa_{1}}\right) \omega_{2} e_{2}-\frac{d \kappa_{1}}{\kappa_{1}^{2}} e_{3}
$$

Então da hipótese $d \kappa_{1} \wedge \omega_{2} \neq 0$ e da Proposição 1.3.8 segue que $Y_{1}$ é uma superfície parametrizada regular com $e_{2}, e_{3}$ campos tangentes e $e_{2} \wedge e_{3}=e_{1}$ campo normal a $Y_{1}$.

Analogamente, usando que $d \kappa_{2} \wedge \omega_{1} \neq 0$, vemos que $Y_{2}$ é uma superfície parametrizada regular com $e_{1}, e_{3}$ campos tangentes e o campo normal na direção de $e_{2}$.

\subsubsection{Superfícies paralelas}

Proposição 3.1.6. Sejam $X: U \subset \mathbb{R}^{2} \rightarrow \mathbb{R}^{3}$ uma superfície parametrizada regular e e e um campo de vetores unitário normal a $X$. Considere a aplicação $\bar{X}: U \subset \mathbb{R}^{2} \rightarrow \mathbb{R}^{3}$ definida por:

$$
\bar{X}(u, v)=X(u, v)+a e_{3}(u, v),
$$

onde a é uma constante real não nula. Denotando por $\mathrm{H}$ e $K$ as curvaturas média e gaussiana de $X$, respectivamente, se $1-2 a \mathrm{H}+a^{2} K \neq 0$, então $\bar{X}$ é uma superfície parametrizada regular. Nesse caso, dizemos que $X$ e $\bar{X}$ são superfícies paralelas à distância a.

Demonstração. Considere $\left\{e_{1}, e_{2}, e_{3}\right\}$ um triedro móvel associado à superfície $X$ e $\omega_{1}, \omega_{2}, \omega_{i j}$, $i, j=1,2,3$, as 1 -formas associadas a ele. Usando as equações (1.3), (1.4) e (1.5), temos que a diferencial de $\bar{X}$ é dada por

$$
d \bar{X}=\left(\omega_{1}-a \omega_{13}\right) e_{1}+\left(\omega_{2}-a \omega_{23}\right) e_{2} .
$$


Assim, utilizando a Proposição 1.3.2, verificamos que

$$
\left(\omega_{1}-a \omega_{13}\right) \wedge\left(\omega_{2}-a \omega_{23}\right)=\left(1-2 a \mathrm{H}+a^{2} K\right) \omega_{1} \wedge \omega_{2} \neq 0,
$$

isto é, as 1-formas $\left(\omega_{1}-a \omega_{13}\right)$ e $\left(\omega_{2}-a \omega_{23}\right)$ são linearmente independentes. Portanto, segue da Proposição 1.3.8 que $\bar{X}$ é uma superfície parametrizada regular, onde $e_{1}$ e $e_{2}$ são campos tangentes a esta superfície e, assim, seu vetor normal está na direção de $e_{3}$.

Observe que dizer que duas superfícies são paralelas à distância $a$ equivale a dizer que entre elas existe uma congruência de retas normal tal que a distância entre os pontos correspondentes é constante e igual a $|a|$.

A seguir, vamos relacionar as curvaturas média e gaussiana da superfície $X$ com as de uma superfície paralela a $X$.

Proposição 3.1.7. Sejam $X(u, v),(u, v) \in U$, uma superfície parametrizada regular e a uma constante real tal que $1-2 a \mathrm{H}+a^{2} K \neq 0$, onde $\mathrm{H}$ e $K$ são, respectivamente, as curvaturas média e gaussiana da superfície $X$. Então as curvaturas $\overline{\mathrm{H}}$ e $\bar{K}$ de uma superfície $\bar{X}$ paralela a $X$ a uma distância a são

$$
\overline{\mathrm{H}}=\frac{\mathrm{H}-a K}{1-2 a \mathrm{H}+a^{2} K} \quad e \quad \bar{K}=\frac{K}{1-2 a \mathrm{H}+a^{2} K} .
$$

Além disso, as curvaturas principais de $\bar{X}$ são dadas por

$$
\bar{\kappa}_{i}=\frac{\kappa_{i}}{1-a \kappa_{i}}, \quad \quad i=1,2 .
$$

Demonstração. Sejam $\left\{e_{1}, e_{2}, e_{3}\right\}$ um triedro móvel associado à superfície $X$ e $\omega_{1}, \omega_{2}, \omega_{i j}$, com $i, j=1,2,3$, as 1 -formas associadas a este triedro móvel. A superfície $\bar{X}$ paralela a $X$ é dada por

$$
\bar{X}=X+a e_{3}
$$

Pela Proposição 3.1.6 temos que

$$
d \bar{X}=\left(\omega_{1}-a \omega_{13}\right) e_{1}+\left(\omega_{2}-a \omega_{23}\right) e_{2}
$$

onde $\left(\omega_{1}-a \omega_{13}\right)$ e $\left(\omega_{2}-a \omega_{23}\right)$ são linearmente independentes pois $1-2 a \mathrm{H}+a^{2} K \neq 0$, por hipótese. Como $\left\langle d \bar{X}, e_{3}\right\rangle=0$, podemos associar a $\bar{X}$ o triedro móvel dado por:

$$
\bar{e}_{i}=e_{i}, \quad i=1,2,3
$$

e denotar por $\bar{\omega}_{1}, \bar{\omega}_{2}, \bar{\omega}_{i j}, i, j=1,2,3$, as 1-formas associadas a este triedro. Das relações (3.5), resulta

$$
d \bar{X}=\bar{\omega}_{1} \bar{e}_{1}+\bar{\omega}_{2} \bar{e}_{2}=\bar{\omega}_{1} e_{1}+\bar{\omega}_{2} e_{2}
$$

e, assim, comparando com (3.4), obtemos

$$
\bar{\omega}_{1}=\omega_{1}-a \omega_{13}, \quad \bar{\omega}_{2}=\omega_{2}-a \omega_{23} .
$$

Além disso, da equação (3.2) temos que

$$
\bar{\omega}_{1} \wedge \bar{\omega}_{2}=\left(1-2 a \mathrm{H}+a^{2} K\right) \omega_{1} \wedge \omega_{2}
$$


e, de (3.5), resulta que

$$
\bar{\omega}_{i j}=\omega_{i j}, \quad i, j=1,2,3 .
$$

Assim, como

$$
\bar{\omega}_{1} \wedge \bar{\omega}_{23}+\bar{\omega}_{13} \wedge \bar{\omega}_{2}=2 \overline{\mathrm{H}} \bar{\omega}_{1} \wedge \bar{\omega}_{2},
$$

usando a Proposição 1.3.2 e as relações (3.7) e (3.8) temos

$$
(\mathrm{H}-a K) \omega_{1} \wedge \omega_{2}=\overline{\mathrm{H}}\left(1-2 a \mathrm{H}+a^{2} K\right) \omega_{1} \wedge \omega_{2} .
$$

Conseqüentemente, como $\omega_{1}$ e $\omega_{2}$ são linearmente independentes, obtemos

$$
\overline{\mathrm{H}}=\frac{\mathrm{H}-a K}{1-2 a \mathrm{H}+a^{2} K} .
$$

Da mesma maneira, como

$$
\bar{\omega}_{12} \wedge \bar{\omega}_{23}=\bar{K} \bar{\omega}_{1} \wedge \bar{\omega}_{2}
$$

usando (3.7) e (3.8), podemos escrever:

$$
K \omega_{1} \wedge \omega_{2}=\bar{K}\left(1-2 a \mathrm{H}+a^{2} K\right) \omega_{1} \wedge \omega_{2},
$$

isto é,

$$
\bar{K}=\frac{K}{1-2 a \mathrm{H}+a^{2} K} .
$$

Desse modo, denotando por $\bar{\kappa}_{1}, \bar{\kappa}_{2}$ as curvaturas principais de $\bar{X}$, temos

$$
\bar{\kappa}_{i}=\overline{\mathrm{H}} \pm \sqrt{\overline{\mathrm{H}}^{2}-\bar{K}}=\frac{\kappa_{i}}{1-a \kappa_{i}}, \quad \quad i=1,2 .
$$

Exemplo 3.1.8. Considere a parametrização da pseudo-esfera dada por:

$$
X(u, v)=(\operatorname{sech} u \cos v, \operatorname{sech} u \operatorname{sen} v, u-\tanh u),
$$

com aplicação normal de Gauss:

$$
N(u, v)=\frac{X_{u} \times X_{v}}{\left\|X_{u} \times X_{v}\right\|}=(-\tanh u \cos v,-\tanh u \operatorname{sen} v,-\operatorname{sech} u) .
$$

No Exemplo A.3.5 vimos que os coeficientes da primeira e da segunda forma quadrática de $X$ são:

$$
\begin{aligned}
E=\tanh ^{2} u, & F=0, \\
e=-\operatorname{sech} u \tanh u, & f=0, \quad g=\operatorname{sech}^{2} u ;
\end{aligned}
$$

de onde obtemos

$$
\mathrm{H}=-\frac{1}{2} \operatorname{coth} u \cosh u, \quad K=-1 .
$$

Assim, considerando constantes $a$ tais que

$$
1-2 a \mathrm{H}+a^{2} K=1-a^{2}+a \operatorname{coth} u \cosh u \neq 0,
$$



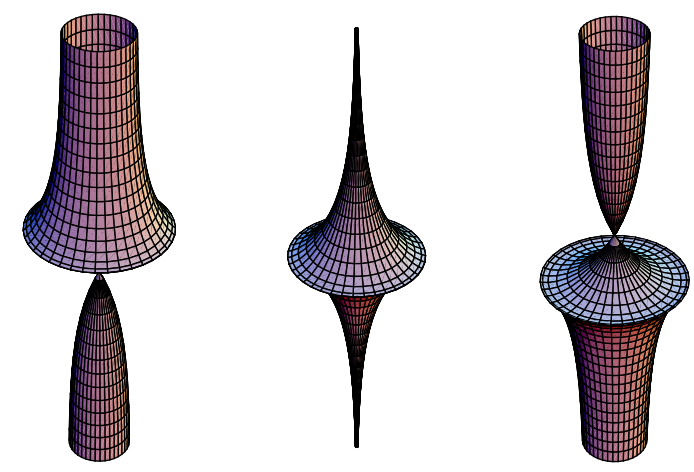

Figura 3.1: Superfícies paralelas à pseudo-esfera (no centro), com respectivas constantes $a$ sendo $-\frac{1}{2}$ e $\frac{1}{2}$.

obtemos a família a um parâmetro de superfícies paralelas à pseudo-esfera, dada por:

$$
\begin{aligned}
X_{a}(u, v)= & X(u, v)+a N(u, v) \\
= & (\operatorname{sech} u \cos v, \operatorname{sech} u \operatorname{sen} v, u-\tanh u) \\
& +a(-\tanh u \cos v,-\tanh u \operatorname{sen} v,-\operatorname{sech} u) .
\end{aligned}
$$

Temos que as curvaturas média e gaussiana de $X_{a}$ são dadas por (3.3):

$$
\mathrm{H}_{a}(u)=\frac{2 a-\operatorname{coth} u \cosh u}{2\left(1-a^{2}+a \operatorname{coth} u \cosh u\right)}, \quad K_{a}(u)=\frac{-1}{1-a^{2}+a \operatorname{coth} u \cosh u} .
$$

Exemplo 3.1.9. Considerando a parametrização da superfície de Enneper dada por:

$$
X(u, v)=\left(u-\frac{u^{3}}{3}+u v^{2}, v-\frac{v^{3}}{3}+v u^{2}, u^{2}-v^{2}\right),
$$

com aplicação normal de Gauss:

$$
N(u, v)=\frac{X_{u} \times X_{v}}{\left\|X_{u} \times X_{v}\right\|}=\left(-2 u, 2 v, 1-u^{2}-v^{2}\right) /\left(1+u^{2}+v^{2}\right),
$$

temos, do Exemplo A.4.4, que os coeficientes da primeira e da segunda forma quadrática de $X$ são:

$$
\begin{gathered}
E=G=\left(1+u^{2}+v^{2}\right)^{2}, \quad F=0, \\
e=2, \quad f=0, \quad g=-2 .
\end{gathered}
$$

Portanto,

$$
\mathrm{H}=0, \quad K=-\frac{4}{\left(1+u^{2}+v^{2}\right)^{4}} .
$$

Assim, para constantes $a$ tais que

$$
1-2 a \mathrm{H}+a^{2} K \neq 0,
$$

ou seja,

$$
\left(1+u^{2}+v^{2}\right)^{4} \neq 4 a^{2}
$$


obtemos a família a um parâmetro de superfícies paralelas à superfície de Enneper, dada por:

$$
\begin{aligned}
X_{a}(u, v)= & \left(u-\frac{u^{3}}{3}+u v^{2}, v-\frac{v^{3}}{3}+v u^{2}, u^{2}-v^{2}\right) \\
& +a\left(-2 u, 2 v, 1-u^{2}-v^{2}\right) /\left(1+u^{2}+v^{2}\right) .
\end{aligned}
$$
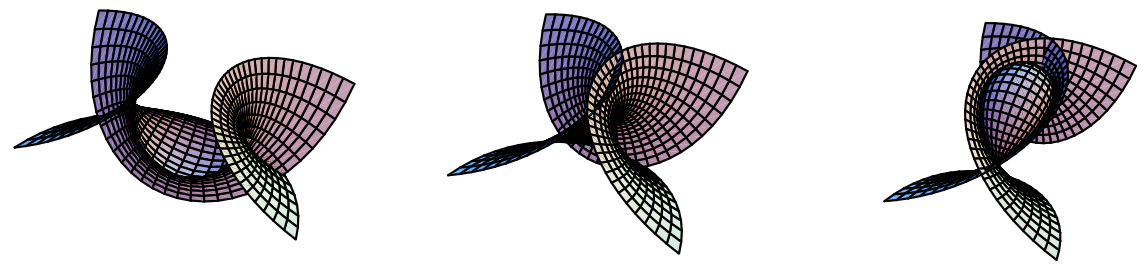

Figura 3.2: Superfícies paralelas à superfície de Enneper (no centro), com respectivas constantes $a$ sendo -3 e 3 .

As curvaturas média e gaussiana da superfície $X_{a}$ são:

$$
\mathrm{H}_{a}=\frac{4 a}{\left(1+u^{2}+v^{2}\right)^{4}-4 a^{2}} \quad \text { e } \quad K_{a}=\frac{4}{4 a^{2}-\left(1+u^{2}+v^{2}\right)^{4}} .
$$

A seguir apresentamos algumas importantes conseqüências da Proposição 3.1.7.

Corolário 3.1.10 (Teorema de Bonnet). A cada superfície de curvatura média constante igual a $c \neq 0$, sem pontos umbílicos e parabólicos, podemos associar duas superfície paralelas, uma de curvatura gaussiana constante igual a $4 c^{2}$ e outra de curvatura média constante igual a $-c$.

Reciprocamente, a cada superfície de curvatura gaussiana constante igual a $4 c^{2}>0$, sem pontos umbilicos, podemos associar duas superfícies paralelas de curvaturas média constante iguais a $c$ e $-c$.

Demonstração. Seja $X: U \subset \mathbb{R}^{2} \rightarrow \mathbb{R}^{3}$ uma superfície parametrizada regular sem pontos umbílicos e parabólicos, de curvatura média constante $\mathrm{H}=c \neq 0$. Defina $X_{1}: U \rightarrow \mathbb{R}^{3}$ por

$$
X_{1}=X+a e_{3}, \quad a=\frac{1}{2 c},
$$

onde $e_{3}$ é um campo unitário normal a $X$. Como a superfície $X$ não possui pontos parabólicos, temos que $K \neq 0$ e assim

$$
1-2 a \mathrm{H}+a^{2} K=\frac{1}{4 c^{2}} K \neq 0 .
$$

Portanto, da Proposição 3.1.7 temos que a curvatura gaussiana da superfície $X_{1}$ é $K_{1}=4 c^{2}$.

Analogamente, definindo $X_{2}: U \rightarrow \mathbb{R}^{3}$ por

$$
X_{2}=X+a e_{3}, \quad a=\frac{1}{c},
$$

temos que

$$
1-2 a \mathrm{H}+a^{2} K=\frac{K-c^{2}}{c^{2}} .
$$

Como a superfície $X$ não possui pontos umbílicos, segue que $0<\mathrm{H}^{2}-K=c^{2}-K$ e, portanto,

$$
1-2 a \mathrm{H}+a^{2} K \neq 0
$$


Logo, novamente da Proposição 3.1.7, segue que a curvatura média da superfície $X_{2}$ é igual a $\mathrm{H}_{2}=-c$.

Reciprocamente, suponha que $X$ seja uma superfície de curvatura gaussiana constante positiva $K=4 c^{2}$, sem pontos umbílicos. Considere as duas superfícies paralelas a $X$ dadas por:

$$
X_{1}=X+\frac{1}{2 c} e_{3} \quad \text { e } \quad X_{2}=X-\frac{1}{2 c} e_{3} .
$$

Como $X$ não tem pontos umbílicos, então

$$
0<\mathrm{H}^{2}-K=\mathrm{H}^{2}-4 c^{2} .
$$

Portanto:

- no primeiro caso, $a=\frac{1}{2 c}, 1-2 a \mathrm{H}+a^{2} K=\frac{2 c-\mathrm{H}}{c} \neq 0$ e a curvatura média de $X_{1}$ é $\mathrm{H}_{1}=-c$;

- no segundo caso, $a=-\frac{1}{2 c}, 2-2 a \mathrm{H}+a^{2} K=\frac{2 c+\mathrm{H}}{c} \neq 0$ e a curvatura média de $X_{2}$ é $\mathrm{H}_{2}=c$.

Isto termina a prova.

Observação 3.1.11. No Teorema de Bonnet, a hipótese de $X$ não ter pontos umbílicos (nem parabólicos) é necessária para garantir a existência de superfícies paralelas satisfazendo a tese do teorema. De fato, a esfera unitária tem curvatura gaussiana constante e igual a 1, porém uma das "superfícies" paralelas à esfera à distância um se reduz a um ponto (o centro da esfera).

Corolário 3.1.12. Sejam $X, \bar{X}: U \subset \mathbb{R}^{2} \rightarrow \mathbb{R}^{3}$ duas superfícies paralelas em $\mathbb{R}^{3}$. Então, $X$ é uma superfície de Weingarten linear se, e somente se, $\bar{X}$ também for uma superfície de Weingarten linear. Em particular, $K=0$ se, e somente se, $\bar{K}=0$.

Demonstração. Suponha que $a \in \mathbb{R}$ seja a distância entre as superfícies $X$ e $\bar{X}$. Segue da Proposição 3.1.7 que as curvaturas média e gaussiana de $\bar{X}$ são dadas por (3.3). Conseqüentemente $\mathrm{H}$ e $K$ satisfazem uma equação linear da forma

$$
\alpha+2 \beta \mathrm{H}+\gamma K=0
$$

se, e somente se, $\overline{\mathrm{H}}$ e $\bar{K}$ satisfizerem

$$
\alpha+2(\beta+\alpha a) \overline{\mathrm{H}}+\left(\gamma+2 \beta a+\alpha a^{2}\right) \bar{K}=0 .
$$

Exemplo 3.1.13. Como a pseudo-esfera (veja Exemplo 3.1.8) tem curvatura gaussiana constante -1 , ela é uma superfície de Weingarten linear, isto é, satisfaz uma relação do tipo (3.14), com $\alpha=\gamma=1$ e $\beta=0$. Portanto, do corolário anterior, segue que a superfície paralela à distância $a$ dada por (3.10) é também de Weingarten linear. De fato, suas curvaturas média e gaussiana (3.11) satisfazem a equação linear do tipo (3.15):

$$
1+2 a \mathrm{H}_{a}+\left(1+a^{2}\right) K_{a}=0 .
$$


Exemplo 3.1.14. Como vimos no Exemplo 3.1.9, a superfície de Enneper é uma superfície mínima. Portanto, ela é uma superfície de Weingarten linear, onde $\alpha=\gamma=0$ e $\beta \in \mathbb{R}-\{0\}$. Assim, do Corolário 3.1.12, segue que a superfície paralela $X_{a}$ (dada por (3.12)) é também de Weingarten linear, com curvaturas média e gaussiana (3.13) satisfazendo

$$
\mathrm{H}_{a}+a K_{a}=0
$$

Corolário 3.1.15. Seja $X(u, v),(u, v) \in U \subset \mathbb{R}^{2}$, uma superfície de Weingarten linear satisfazendo

$$
\alpha+2 \beta \mathrm{H}+\gamma K=0
$$

onde $\beta, \gamma \neq 0$.

(i) Se $\alpha=0$, então $X$ é paralela a uma superfície mínima.

(ii) Se $\alpha \neq 0$, então $X$ é paralela a uma superfície de curvatura gaussiana constante.

Demonstração. Sejam $X$ uma superfície de Weingarten linear e $\bar{X}$ uma superfície paralela a $X$ a uma distância $a$, ou seja,

$$
\bar{X}=X+a e_{3},
$$

onde $e_{3}$ é um campo unitário normal a $X$. Então,

(i) Se $\alpha=0$, considere a distância $a=-\gamma /(2 \beta)$. Então, segue de (3.15) que a curvatura média de $\bar{X}$ é nula.

(ii) Se $\alpha \neq 0$, considere a distância $a=-\beta / \alpha$. Então, de (3.15), resulta que

$$
\alpha^{2}+\left(\alpha \gamma-\beta^{2}\right) \bar{K}=0,
$$

ou seja $\bar{K}$ é constante.

\subsection{Congruências pseudo-esféricas}

Desenvolveremos, nesta seção, a teoria das congruências pseudo-esféricas obtida entre superfícies pseudo-esféricas, isto é, de curvatura gaussiana constante negativa, como veremos no Teorema de Bäcklund. Estas congruências deram origem à transformação de Bäcklund, que permite obter novas soluções da equação de Sine-Gordon,

$$
\phi_{u u}-\phi_{v v}=\operatorname{sen} \phi \cos \phi
$$

a partir de uma que é dada.

Definição 3.2.1. Considere $S$ e $\bar{S}$ duas superfícies parametrizadas regulares de $\mathbb{R}^{3}$ e $l: S \rightarrow \bar{S}$ um difeomorfismo tal que $l(p) \neq p$, para todo $p \in S$. Dizemos que a congruência de retas $l$ é pseudo-esférica se

(i) para todo $p \in S$, a reta determinada por $p$ e $\bar{p}=l(p)$ tangencia as superfícies $S$ e $\bar{S}$, respectivamente, em $p$ e $\bar{p}$; 
(ii) a distância entre os pontos $p$ e $\bar{p}$ é igual a uma constante $r>0$ que independe de $p$;

(iii) o ângulo entre os vetores normais às superfícies $S$ e $\bar{S}$ em seus respectivos pontos $p$ e $\bar{p}$ é igual à constante $\theta \in(0, \pi)$, que independe de $p$.

O próximo resultado nos diz que congruências de retas pseudo-esféricas só existem entre superfícies de mesma curvatura gaussiana constante negativa, o que justifica o nome dado a este tipo de congruência. Mais precisamente,

Teorema 3.2.2 (Teorema de Bäcklund). Seja $l: S \rightarrow \bar{S}$ uma congruência de retas pseudoesférica entre as superfícies regulares $S$ e $\bar{S}$ tal que a distância entre os pontos correspondentes seja igual à constante $r>0$ e o ângulo entre as retas normais desses pontos seja $\theta$, onde $0<\theta<\pi$. Então as superfícies $S$ e $\bar{S}$ têm mesma curvatura gaussiana constante igual a

$$
K=\bar{K}=-\frac{\operatorname{sen}^{2} \theta}{r^{2}} .
$$

Demonstração. Apresentaremos a seguir a prova dada por S. Chern e C. Terng em [15].

Sejam $S$ e $\bar{S}$ superfícies parametrizadas por $X(u, v)$ e $\bar{X}(u, v)$, respectivamente, definidas num aberto $U$ de $\mathbb{R}^{2}$. Considere $\left\{e_{1}, e_{2}, e_{3}\right\}$ um triedro móvel associado à superfície $X$ e $\omega_{1}, \omega_{2}, \omega_{i j}$, $i, j=1,2,3$, as 1 -formas associadas a este triedro. Supondo que $e_{1}$ está na direção da reta determinada pelos pontos correspondentes de $S$ e $\bar{S}$, isto é,

$$
e_{1}=\frac{l(X(u, v))-X(u, v)}{\|l(X(u, v))-X(u, v)\|}
$$

temos que:

$$
\bar{X}(u, v)=X(u, v)+r e_{1}(u, v) .
$$

Note que, da definição de congruência pseudo-esférica, o campo de vetores $e_{1}$ é tangente também à superfície $\bar{X}$. Assim, podemos associar a $\bar{X}$ o seguinte triedro móvel:

$$
\begin{aligned}
& \bar{e}_{1}=e_{1}, \\
& \bar{e}_{2}=\cos \theta e_{2}+\operatorname{sen} \theta e_{3}, \\
& \bar{e}_{3}=-\operatorname{sen} \theta e_{2}+\cos \theta e_{3},
\end{aligned}
$$

onde $\theta$ é o ângulo formado pelos compos normais $e_{3}$ e $\bar{e}_{3}$. Denote por $\bar{\omega}_{1}, \bar{\omega}_{2}, \bar{\omega}_{i j}$ as 1-formas associadas ao triedro móvel de $\bar{X}$. De (3.18) obtemos

$$
d \bar{X}=\omega_{1} e_{1}+\left(\omega_{2}+r \omega_{12}\right) e_{2}+r \omega_{13} e_{3}
$$

Por outro lado, usando (3.19), temos

$$
\begin{aligned}
d \bar{X} & =\bar{\omega}_{1} \bar{e}_{1}+\bar{\omega}_{2} \bar{e}_{2} \\
& =\bar{\omega}_{1} e_{1}+\bar{\omega}_{2} \cos \theta e_{2}+\bar{\omega}_{2} \operatorname{sen} \theta e_{3},
\end{aligned}
$$

de onde concluímos que

$$
\begin{aligned}
& \bar{\omega}_{1}=\omega_{1}, \\
& \bar{\omega}_{2} \cos \theta=\omega_{2}+r \omega_{12}, \\
& \bar{\omega}_{2} \operatorname{sen} \theta=r \omega_{13} .
\end{aligned}
$$


Das duas últimas equações, temos

$$
\omega_{12}=-\frac{1}{r} \omega_{2}+\cot \theta \omega_{13}
$$

e, portanto, das equações de estrutura segue que

$$
\begin{aligned}
d \omega_{12} & =-\frac{1}{r} d \omega_{2}+\cot \theta d \omega_{13}=\omega_{12} \wedge\left(\frac{1}{r} \omega_{1}+\cot \theta \omega_{23}\right) \\
& =\left(-\frac{1}{r} \omega_{2}+\cot \theta \omega_{13}\right) \wedge\left(\frac{1}{r} \omega_{1}+\cot \theta \omega_{23}\right)=\left(\frac{1}{r^{2}}+K \cot ^{2} \theta\right) \omega_{1} \wedge \omega_{2} .
\end{aligned}
$$

Assim, resulta da equação de Gauss:

$$
d \omega_{12}=-K \omega_{1} \wedge \omega_{2}
$$

que a curvatura gaussiana de $X$ é dada por:

$$
K=-\frac{\operatorname{sen}^{2} \theta}{r^{2}}
$$

Calcularemos agora curvatura gaussiana da superfície $\bar{X}$. Das relações (3.19) segue que

$$
\begin{aligned}
& \bar{\omega}_{12}=\left\langle d \bar{e}_{1}, \bar{e}_{2}\right\rangle=\cos \theta \omega_{12}+\operatorname{sen} \theta \omega_{13}, \\
& \bar{\omega}_{13}=\left\langle d \bar{e}_{1}, \bar{e}_{3}\right\rangle=-\operatorname{sen} \theta \omega_{12}+\cos \theta \omega_{13}, \\
& \bar{\omega}_{23}=\left\langle d \bar{e}_{2}, \bar{e}_{3}\right\rangle=\omega_{23} .
\end{aligned}
$$

Portanto, usando a equação (3.21) e as equações de estrutura, obtemos

$$
\begin{aligned}
d \bar{\omega}_{12} & =\cos \theta d \omega_{12}+\operatorname{sen} \theta d \omega_{13} \\
& =-\frac{1}{r} \cos \theta \omega_{1} \wedge \omega_{12}+\frac{1}{\operatorname{sen} \theta} \omega_{12} \wedge \omega_{23} \\
& =\omega_{12} \wedge\left(\frac{\cos \theta}{r} \omega_{1}+\frac{1}{\operatorname{sen} \theta} \omega_{23}\right) \\
& =\frac{\cos \theta}{r^{2}} \omega_{1} \wedge \omega_{2}+\frac{\operatorname{sen} \theta}{r} \omega_{1} \wedge \omega_{13}+\frac{\cos \theta}{\operatorname{sen}^{2} \theta} \omega_{13} \wedge \omega_{23} .
\end{aligned}
$$

Como $\omega_{13} \wedge \omega_{23}=K \omega_{1} \wedge \omega_{2}=-\frac{\operatorname{sen}^{2} \theta}{r^{2}} \omega_{1} \wedge \omega_{2}$, resulta que

$$
d \bar{\omega}_{12}=\frac{\operatorname{sen} \theta}{r} \omega_{1} \wedge \omega_{13}
$$

Por outro lado, da equação de Gauss, temos

$$
d \bar{\omega}_{12}=-\bar{K} \bar{\omega}_{1} \wedge \bar{\omega}_{2}=-\bar{K} \frac{r}{\operatorname{sen} \theta} \omega_{1} \wedge \omega_{13},
$$

ou seja,

$$
\bar{K}=-\frac{\operatorname{sen}^{2} \theta}{r^{2}} .
$$


Nesta demonstração, vimos que a existência de uma congruência pseudo-esférica entre duas superfícies implica na equação

$$
\omega_{12}=-\frac{1}{r} \omega_{2}+\cot \theta \omega_{13},
$$

denominada transformação de Bäcklund. Esta equação será denotada por $B(\theta)$ para indicar sua dependência apenas do parâmetro $\theta$, sendo que $r=\operatorname{sen} \theta / \sqrt{-K}$.

Proposição 3.2.3. Congruências pseudo-esféricas preservam direções principais e direções conjugadas. Conseqüentemente, preservam também linhas de curvatura e linhas assintóticas.

Demonstração. Considere $X, \bar{X}: U \subset \mathbb{R}^{2} \rightarrow \mathbb{R}^{3}$ superfícies parametrizadas regulares relacionadas por uma congruência pseudo-esférica como feito na prova do Teorema 3.2.2. Considere o triedro móvel $\left\{e_{1}, e_{2}, e_{3}\right\}$ associado a $X$ e $\left\{\bar{e}_{1}, \bar{e}_{2}, \bar{e}_{3}\right\}$ associado a $\bar{X}$ definido por (3.19). Logo, verificam-se as relações (3.20), (3.21) e (3.22).

A prova que direções principais são preservadas segue da Proposição 1.3 .1 e do fato que

$$
\begin{aligned}
\bar{\omega}_{1} \bar{\omega}_{23}-\bar{\omega}_{2} \bar{\omega}_{13} & =\omega_{1} \omega_{23}-\frac{r}{\operatorname{sen} \theta} \omega_{13}\left(-\operatorname{sen} \theta \omega_{12}+\cos \theta \omega_{13}\right) \\
& =\omega_{1} \omega_{23}+r \omega_{13}\left(-\frac{1}{r} \omega_{2}+\cot \theta \omega_{13}\right)-r \cos \theta \omega_{13}^{2} \\
& =\omega_{1} \omega_{23}-\omega_{13} \omega_{2},
\end{aligned}
$$

onde na segunda igualdade usamos (3.21).

Agora, vamos mostrar que este tipo de congruência também preserva as direções conjugadas. Para isso, verificaremos que as segundas formas quadráticas das superfícies $X$ e $\bar{X}$ são proporcionais. Utilizando as relações (1.12), (3.20), (3.21) e (3.22), temos

$$
\begin{aligned}
\overline{\mathrm{II}} & =\bar{\omega}_{1} \bar{\omega}_{13}+\bar{\omega}_{2} \bar{\omega}_{23} \\
& =\omega_{1}\left(-\operatorname{sen} \theta \omega_{12}+\cos \theta \omega_{13}\right)+\frac{r}{\operatorname{sen} \theta} \omega_{13} \omega_{23} \\
& =-\operatorname{sen} \theta \omega_{1}\left(-\frac{1}{r} \omega_{2}+\cot \theta \omega_{13}\right)+\cos \theta \omega_{1} \omega_{13}+\frac{r}{\operatorname{sen} \theta} \omega_{13} \omega_{23} \\
& =\frac{\operatorname{sen} \theta}{r} \omega_{1} \omega_{2}+\frac{r}{\operatorname{sen} \theta} \omega_{13} \omega_{23} .
\end{aligned}
$$

Assim, substituindo na relação anterior

$$
\omega_{13}=h_{11} \omega_{1}+h_{12} \omega_{2}, \quad \omega_{23}=h_{21} \omega_{1}+h_{22} \omega_{2}
$$

e lembrando que $h_{12}=h_{21}$, resulta que

$$
\overline{\mathrm{II}}=\frac{\operatorname{sen} \theta}{r} \omega_{1} \omega_{2}+\frac{r}{\operatorname{sen} \theta}\left[h_{12} \mathrm{II}+\left(h_{11} h_{22}-h_{12}^{2}\right) \omega_{1} \omega_{2}\right],
$$

onde II é a segunda forma quadrática de $X$. Como, do Teorema 3.2.2, temos

$$
h_{11} h_{22}-h_{12}^{2}=K=-\frac{\operatorname{sen}^{2} \theta}{r^{2}},
$$

obtemos

$$
\overline{\mathrm{II}}=\frac{r}{\operatorname{sen} \theta} h_{12} \mathrm{II} .
$$

Isto prova que as direções conjugadas são preservadas e, conseqüentemente, as linhas assintóticas também são. 
O próximo resultado mostra que, integrando $B(\theta)$, obtemos, a partir de uma dada superfície $S$ pseudo-esférica, satisfazendo (3.17), uma família a dois parâmetros de superfícies desse tipo (localmente) relacionadas a $S$ por congruências pseudo-esféricas.

Teorema 3.2.4 (Teorema de Integrabilidade). Seja $X: U \subset \mathbb{R}^{2} \rightarrow S \subset \mathbb{R}^{3}$ uma superfície parametrizada regular de curvatura gaussiana constante igual a

$$
K=-\frac{\operatorname{sen}^{2} \theta}{r^{2}}
$$

onde $r>0$ e $0<\theta<\pi$ são constantes. Considere um ponto $q_{0} \in U$ e $w_{0} \in T_{X\left(q_{0}\right)} S$ um vetor unitário, que não é um vetor principal. Então, existe uma única superfície $\bar{S}$ e uma congruência pseudo-esférica l definida numa vizinhança de $p_{0}=X\left(q_{0}\right)$ em $S$ tal que $l\left(p_{0}\right)=p_{0}+r w_{0}$ e $\theta$ é $o$ ângulo entre os vetores normais às superfícies nos pontos $p_{0}$ e $l\left(p_{0}\right)$.

Demonstração. Como $X$ é uma superfície parametrizada regular de curvatura gaussiana constante $K<0$, da Proposição 2.3.3, segue que suas formas quadráticas são

$$
\begin{aligned}
\mathrm{I} & =-\frac{1}{K}\left(\cos ^{2} \phi d u^{2}+\operatorname{sen}^{2} \phi d v^{2}\right), \\
\mathrm{II} & =\frac{1}{\sqrt{-K}} \operatorname{sen} \phi \cos \phi\left(d u^{2}-d v^{2}\right),
\end{aligned}
$$

onde $\phi$ é solução da equação de Sine-Gordon, $\phi_{u u}-\phi_{v v}=\operatorname{sen} \phi \cos \phi$, associada a $X$. Seja $\left\{\tilde{e}_{1}, \tilde{e}_{2}, \tilde{e}_{3}\right\}$ um triedro móvel principal associado a $X$ e denote por $\tilde{\omega}_{1}, \tilde{\omega}_{2}, \tilde{\omega}_{i j}, i, j=1,2,3$, as 1 -formas associadas a este triedro. Como $X$ é uma parametrização principal, podemos considerar

$$
\tilde{e}_{1}=\frac{\sqrt{-K}}{\cos \phi} X_{u}, \quad \tilde{e}_{2}=\frac{\sqrt{-K}}{\operatorname{sen} \phi} X_{v}
$$

de onde, como na Proposição 1.3.3, temos que

$$
\begin{aligned}
\tilde{\omega}_{1} & =\frac{1}{\sqrt{-K}} \cos \phi d u, \\
\tilde{\omega}_{2} & =\frac{1}{\sqrt{-K}} \operatorname{sen} \phi d v, \\
\tilde{\omega}_{12} & =\phi_{v} d u+\phi_{u} d v, \\
\tilde{\omega}_{13} & =\operatorname{sen} \phi d u, \\
\tilde{\omega}_{23} & =-\cos \phi d v .
\end{aligned}
$$

Considere, agora, o triedro móvel associado a $X$ dado por:

$$
\begin{aligned}
& e_{1}=\cos \bar{\phi} \tilde{e}_{1}+\operatorname{sen} \bar{\phi} \tilde{e}_{2}, \\
& e_{2}=-\operatorname{sen} \bar{\phi} \tilde{e}_{1}+\cos \bar{\phi} \tilde{e}_{2}, \\
& e_{3}=\tilde{e}_{3} .
\end{aligned}
$$

Então, da Observação 1.1.2, as 1-formas $\omega_{1}, \omega_{2}, \omega_{i j}, i, j=1,2,3$, associadas a este triedro são

$$
\begin{aligned}
\omega_{1} & =\cos \bar{\phi} \tilde{\omega}_{1}+\operatorname{sen} \bar{\phi} \tilde{\omega}_{2}, \\
\omega_{2} & =-\operatorname{sen} \bar{\phi} \tilde{\omega}_{1}+\cos \bar{\phi} \tilde{\omega}_{2}, \\
\omega_{12} & =d \bar{\phi}+\tilde{\omega}_{12}, \\
\omega_{13} & =\cos \bar{\phi} \tilde{\omega}_{13}+\operatorname{sen} \bar{\phi} \tilde{\omega}_{23}, \\
\omega_{23} & =-\operatorname{sen} \bar{\phi} \tilde{\omega}_{13}+\cos \bar{\phi} \tilde{\omega}_{23} .
\end{aligned}
$$


Observe que construir uma congruência pseudo-esférica $l$ com constante $\theta$ é equivalente a encontrar um triedro móvel $\left\{e_{1}, e_{2}, e_{3}\right\}$ em $X$ tal que $e_{1}\left(q_{0}\right)=w_{0}$ e cujas 1-formas correspondentes $\omega_{1}, \omega_{2}, \omega_{i j}, i, j=1,2,3$, satisfazem a transformação de Bäcklund

$$
\omega_{12}=-\frac{1}{r} \omega_{2}+\cot \theta \omega_{13}
$$

Assim, substituindo (3.24) em (3.25), temos

$$
d \bar{\phi}+\tilde{\omega}_{12}=-\frac{1}{r}\left(-\operatorname{sen} \bar{\phi} \tilde{\omega}_{1}+\cos \bar{\phi} \tilde{\omega}_{2}\right)+\cot \theta\left(\cos \bar{\phi} \tilde{\omega}_{13}+\operatorname{sen} \bar{\phi} \tilde{\omega}_{23}\right) .
$$

Segue de (3.23) que

$$
\begin{aligned}
d \bar{\phi}+\phi_{v} d u+\phi_{u} d v= & \frac{\operatorname{sen} \bar{\phi}}{r \sqrt{-K}} \cos \phi d u-\frac{\cos \bar{\phi}}{r \sqrt{-K}} \operatorname{sen} \phi d v+ \\
& +\cot \theta \cos \bar{\phi} \operatorname{sen} \phi d u-\cot \theta \operatorname{sen} \bar{\phi} \cos \phi d v
\end{aligned}
$$

e, como $-K=\frac{\operatorname{sen}^{2} \theta}{r^{2}}$, obtemos (da independência linear de $d u$ e $d v$ ) que

$$
\left\{\begin{array}{l}
\bar{\phi}_{u}+\phi_{v}=\operatorname{sen} \bar{\phi} \cos \phi \csc \theta+\cot \theta \cos \bar{\phi} \operatorname{sen} \phi \\
\bar{\phi}_{v}+\phi_{u}=-\cos \bar{\phi} \operatorname{sen} \phi \csc \theta-\cot \theta \operatorname{sen} \bar{\phi} \cos \phi
\end{array}\right.
$$

Portanto, encontrar um campo de vetores unitário $e_{1}$ tal que $l(p)=p+r e_{1}$ é uma congruência pseudo-esférica é equivalente a integrar o sistema de equações diferenciais parciais (3.26). Como

$$
\left\{\begin{aligned}
\bar{\phi}_{u v}+\phi_{v v}= & (\cos \bar{\phi} \cos \phi \csc \theta-\cot \theta \operatorname{sen} \bar{\phi} \operatorname{sen} \phi) \bar{\phi}_{v} \\
& -(\operatorname{sen} \bar{\phi} \operatorname{sen} \phi \csc \theta-\cot \theta \cos \bar{\phi} \cos \phi) \phi_{v} \\
\bar{\phi}_{v u}+\phi_{u u}= & (\operatorname{sen} \bar{\phi} \operatorname{sen} \phi \csc \theta-\cot \theta \cos \bar{\phi} \cos \phi) \bar{\phi}_{u} \\
& -(\cos \bar{\phi} \cos \phi \csc \theta-\cot \theta \operatorname{sen} \bar{\phi} \operatorname{sen} \phi) \phi_{u}
\end{aligned}\right.
$$

resulta que

$$
\begin{aligned}
\bar{\phi}_{u v}-\bar{\phi}_{v u}+\phi_{v v}-\phi_{u u}= & (\cos \bar{\phi} \cos \phi \csc \theta-\cot \theta \operatorname{sen} \bar{\phi} \operatorname{sen} \phi)\left(\bar{\phi}_{v}+\phi_{u}\right) \\
& -(\operatorname{sen} \bar{\phi} \operatorname{sen} \phi \csc \theta-\cot \theta \cos \bar{\phi} \cos \phi)\left(\bar{\phi}_{u}+\phi_{v}\right) \\
= & -\operatorname{sen} \phi \cos \phi\left(\csc ^{2} \theta-\cot ^{2} \theta\right)=-\operatorname{sen} \phi \cos \phi
\end{aligned}
$$

Ou seja, a condição de integrabilidade do sistema (3.26), isto é, $\bar{\phi}_{u v}=\bar{\phi}_{v u}$, é equivalente à equação de Sine-Gordon

$$
\phi_{u u}-\phi_{v v}=\operatorname{sen} \phi \cos \phi .
$$

Logo, pelo Teorema de Frobenius, existe um triedro móvel $\left\{e_{1}, e_{2}, e_{3}\right\}$ definido em uma vizinhança $U_{0}$ de $q_{0}$, satisfazendo a transformação de Bäcklund e tal que $e_{1}\left(q_{0}\right)=w_{0}$. Como $w_{0}$ não é direção principal, podemos supor que $U_{0}$ é uma vizinhança suficientemente pequena tal que $e_{1}(q)$ não é uma direção principal para todo $q \in U_{0}$.

Considere a aplicação $\bar{X}: U_{0} \subset \mathbb{R}^{2} \rightarrow \mathbb{R}^{3}$ tal que

$$
\bar{X}(u, v)=X(u, v)+r e_{1}(u, v) .
$$


Vamos mostrar que $\bar{X}$ é uma superfície parametrizada regular e que a aplicação $l$, que associa $X(u, v)$ a $\bar{X}(u, v)$, para cada $(u, v) \in U_{0}$, é uma congruência pseudo-esférica.

Usando a transformação de Bäcklund obtemos

$$
\begin{aligned}
d \bar{X} & =d X+r d e_{1} \\
& =\omega_{1} e_{1}+\left(\omega_{2}+r \omega_{12}\right) e_{2}+r \omega_{13} e_{3} \\
& =\omega_{1} e_{1}+\left[\omega_{2}+r\left(-\frac{1}{r} \omega_{2}+\cot \theta \omega_{13}\right)\right] e_{2}+r \omega_{13} e_{3},
\end{aligned}
$$

isto é,

$$
d \bar{X}=\omega_{1} e_{1}+\frac{r}{\operatorname{sen} \theta} \omega_{13}\left(\cos \theta e_{2}+\operatorname{sen} \theta e_{3}\right) .
$$

Como $e_{1}$ não é uma direção principal, segue da Proposição 1.3.6 que as 1-formas $\omega_{1}$ e $\omega_{13}$ são linearmente independentes. Portanto, da Proposição 1.3.8, temos que $\bar{X}$ é uma superfície parametrizada regular e os campos $e_{1}$ e $\left(\cos \theta e_{2}+\operatorname{sen} \theta e_{3}\right)$ são tangentes a $\bar{X}$. Logo $e_{1}$ é tangente comum a $X$ e $\bar{X}$. Além disso, sendo

$$
e_{1} \wedge\left(\cos \theta e_{2}+\operatorname{sen} \theta e_{3}\right)=-\operatorname{sen} \theta e_{2}+\cos \theta e_{3}
$$

campo normal a $\bar{X}$, o ângulo entre este e o campo normal a $X$ é $\theta$. Assim, $l$ é uma congruência pseudo-esférica entre $X$ e $\bar{X}$ tal que a distância entre os pontos destas superfícies é igual à constante $r$.

A superfície $\bar{S}$ é dita transformada de Bäcklund da superfície $S$. Observe que os dois parâmetros da família de superfícies de mesma curvatura negativa constante são $\theta$ e aquele que corresponde à escolha do vetor unitário $w_{0}$.

\subsection{Transformação de Bäcklund para a equação de Sine-Gordon}

Na Proposição 2.3.3 vimos que as superfícies pseudo-esféricas correspondem a soluções não nulas da equação de Sine-Gordon. Na Proposição 3.3.1 veremos como a transformação de Bäcklund fornece um método para a construção de novas soluções desta equação a partir de uma já conhecida.

Proposição 3.3.1. Seja $\phi(u, v)$ uma solução da equação de Sine-Gordon

$$
\phi_{u u}-\phi_{v v}=\operatorname{sen} \phi \cos \phi
$$

e $\theta$ uma constante. Então o sistema

$$
\left\{\begin{array}{c}
\bar{\phi}_{u}+\phi_{v}=\operatorname{sen} \bar{\phi} \cos \phi \csc \theta+\cot \theta \cos \bar{\phi} \operatorname{sen} \phi \\
\bar{\phi}_{v}+\phi_{u}=-\cos \bar{\phi} \operatorname{sen} \phi \csc \theta-\cot \theta \operatorname{sen} \bar{\phi} \cos \phi
\end{array}\right.
$$

é integrável. Além disso, $\bar{\phi}$ é solução da equação de Sine-Gordon.

Demonstração. Faremos a prova desta proposição de duas maneiras: analiticamente e geometricamente. Vamos primeiro apresentar a prova geométrica. 
Considere $\phi$ uma solução não nula da equação de Sine-Gordon. Pela Proposição 2.3.3, existe uma superfície parametrizada regular $X(u, v),(u, v) \in U$, associada a $\phi$ e de curvatura gaussiana $K=-1$, determinada pelas formas quadráticas

$$
\begin{aligned}
\mathrm{I} & =\cos ^{2} \phi d u^{2}+\operatorname{sen}^{2} \phi d v^{2} \\
\mathrm{II} & =\operatorname{sen} \phi \cos \phi\left(d u^{2}-d v^{2}\right) .
\end{aligned}
$$

Do Teorema da Integrabilidade, fixado um vetor unitário não principal $w_{0} \in \mathrm{T}_{X\left(q_{0}\right)} X, q_{0} \in U$, existe um triedro móvel $\left\{e_{1}, e_{2}, e_{3}\right\}$ associado a $X$, definido numa vizinhança $U_{0} \subset \mathbb{R}^{2}$ de $q_{0}$, tal que $e_{1}\left(q_{0}\right)=w_{0}$ e que satisfaz a transformação de Bäcklund. Mais ainda, a aplicação $\bar{X}: U_{0} \rightarrow \mathbb{R}^{3}$ dada por

$$
\bar{X}=X+r e_{1}=X+r\left(\cos \bar{\phi} \tilde{e}_{1}+\operatorname{sen} \bar{\phi} \tilde{e}_{2}\right),
$$

onde $r=\operatorname{sen} \theta$, é uma superfície parametrizada regular de curvatura gaussiana $\bar{K}=K=-1$, sendo $\tilde{e}_{1}, \tilde{e}_{2}$ campos de direções principais. Além disso, de (3.20), (3.23) e (3.24), temos que

$$
\begin{aligned}
\overline{\mathrm{I}} & =\bar{\omega}_{1}^{2}+\bar{\omega}_{2}^{2}=\omega_{1}^{2}+\omega_{13}^{2} \\
& =\left(\cos \bar{\phi} \tilde{\omega}_{1}+\operatorname{sen} \bar{\phi} \tilde{\omega}_{2}\right)^{2}+\left(\cos \bar{\phi} \tilde{\omega}_{13}+\operatorname{sen} \bar{\phi} \tilde{\omega}_{23}\right)^{2} \\
& =\cos ^{2} \bar{\phi} d u^{2}+\operatorname{sen}^{2} \bar{\phi} d v^{2} .
\end{aligned}
$$

Da mesma forma, usando também (3.22), resulta

$$
\begin{aligned}
\overline{\mathrm{II}}= & \bar{\omega}_{1} \bar{\omega}_{13}+\bar{\omega}_{2} \bar{\omega}_{23}=\omega_{1}\left(-\operatorname{sen} \theta \omega_{12}+\cos \theta \omega_{13}\right)+\omega_{13} \omega_{23} \\
= & \omega_{1} \omega_{2}+\omega_{13} \omega_{23} \\
= & \left(\cos \bar{\phi} \tilde{\omega}_{1}+\operatorname{sen} \bar{\phi} \tilde{\omega}_{2}\right)\left(-\operatorname{sen} \bar{\phi} \tilde{\omega}_{1}+\cos \bar{\phi} \tilde{\omega}_{2}\right) \\
& +\left(\cos \bar{\phi} \tilde{\omega}_{13}+\operatorname{sen} \bar{\phi} \tilde{\omega}_{23}\right)\left(-\operatorname{sen} \bar{\phi} \tilde{\omega}_{13}+\cos \bar{\phi} \tilde{\omega}_{23}\right) \\
= & \operatorname{sen} \bar{\phi} \cos \bar{\phi}\left(-d u^{2}+d v^{2}\right),
\end{aligned}
$$

onde na terceira igualdade usamos a transformação de Bäcklund, $\omega_{12}=-\frac{1}{r} \omega_{2}+\cot \theta \omega_{13}$. Portanto, as formas quadráticas da superfície $\bar{X}$ são:

$$
\begin{aligned}
\overline{\mathrm{I}} & =\cos ^{2} \bar{\phi} d u^{2}+\operatorname{sen}^{2} \bar{\phi} d v^{2}, \\
\overline{\mathrm{II}} & =\operatorname{sen} \bar{\phi} \cos \bar{\phi}\left(-d u^{2}+d v^{2}\right) .
\end{aligned}
$$

Segue, assim, da equação de Gauss, que $\bar{\phi}$ é também solução da equação de Sine-Gordon.

Para demonstrar esta proposição analiticamente, basta derivar a primeira equação do sistema (3.29) com relação a variável $v$, a segunda com relação a variável $u$, obtendo, respectivamente:

$$
\begin{aligned}
\bar{\phi}_{u v}+\phi_{v v} & =(\cos \bar{\phi} \cos \phi \csc \theta-\cot \theta \operatorname{sen} \bar{\phi} \operatorname{sen} \phi) \bar{\phi}_{v}-(\operatorname{sen} \bar{\phi} \operatorname{sen} \phi \csc \theta-\cot \theta \cos \bar{\phi} \cos \phi) \phi_{v}, \\
\bar{\phi}_{v u}+\phi_{u u} & =(\operatorname{sen} \bar{\phi} \operatorname{sen} \phi \csc \theta-\cot \theta \cos \bar{\phi} \cos \phi) \bar{\phi}_{u}-(\cos \bar{\phi} \cos \phi \csc \theta-\cot \theta \operatorname{sen} \bar{\phi} \operatorname{sen} \phi) \phi_{u} .
\end{aligned}
$$

Assim, subtraindo a primeira equação da segunda e utilizando a transformação de Bäcklund, obtemos que $\bar{\phi}_{v u}=\bar{\phi}_{u v}$. Conseqüentemente pelo Teorema de Frobenius, fixada a condição inicial $\bar{\phi}\left(u_{0}, v_{0}\right)$, existe uma única solução $\bar{\phi}$ do sistema (3.29) definida numa vizinhança de $\left(u_{0}, v_{0}\right)$. Além disso, $\bar{\phi}$ é solução da equação de Sine-Gordon. De fato, segue da equação de Gauss que

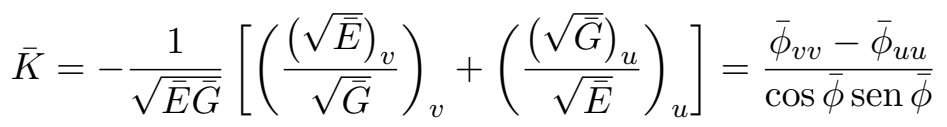


e, como $\bar{X}$ possui curvatura gaussiana $\bar{K}=-1$, obtemos

$$
\bar{\phi}_{u u}-\bar{\phi}_{v v}=\operatorname{sen} \bar{\phi} \cos \bar{\phi}
$$

Observação 3.3.2. Segue da prova do Teorema de Integrabilidade que a transformação de Bäcklund

$$
\omega_{12}=-\frac{1}{r} \omega_{2}+\cot \theta \omega_{13}
$$

é equivalente ao sistema (3.29), o qual também nos referimos por $B(\theta)$, onde $0<\theta<\pi$.

A solução $\bar{\phi}$, obtida integrando a transformação de Bäcklund (3.29) com constante $\theta$, é denominada solução associada a $\phi$ por $B(\theta)$ :

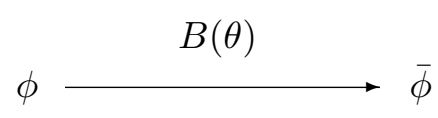

Figura 3.3: Diagrama da transformação de Bäcklund.

A seguir vamos obter a superfície de Kuen como transformada de Bäcklund da pseudo-esfera.

Exemplo 3.3.3 (Transformada de Bäcklund da pseudo-esfera). Seja $X(u, v)$ a parametrização da pseudo-esfera dada por:

$$
X(u, v)=(\operatorname{sech} u \cos v, \operatorname{sech} u \operatorname{sen} v, u-\tanh u) .
$$

No Exemplo 2.3.6, vimos que a função $\phi(u)$ definida por

$$
\cos \phi=\tanh u, \quad \operatorname{sen} \phi=\operatorname{sech} u
$$

é uma solução da equação de Sine-Gordon. Derivando estas relações com relação a $u$ e $v$, obtemos

$$
\begin{aligned}
\phi_{u} & =-\operatorname{sech} u, \\
\phi_{v} & =0 .
\end{aligned}
$$

Logo, segue da Proposição 3.3.1 que para obter outras soluções $\bar{\phi}$ da equação de Sine-Gordon, basta integrar a transformação de Bäcklund:

$$
\left\{\begin{array}{l}
\bar{\phi}_{u}=\operatorname{sen} \bar{\phi} \tanh u \csc \theta+\operatorname{coth} \theta \cos \bar{\phi} \operatorname{sech} u \\
\bar{\phi}_{v}-\operatorname{sech} u=-\cos \bar{\phi} \operatorname{sech} u \csc \theta-\cot \theta \operatorname{sen} \bar{\phi} \tanh u
\end{array} \quad \theta \in \mathbb{R}\right.
$$

Considerando $\theta=\frac{\pi}{2}$, temos o sistema

$$
\left\{\begin{array}{l}
\bar{\phi}_{u}=\operatorname{sen} \bar{\phi} \tanh u \\
\bar{\phi}_{v}=(1-\cos \bar{\phi}) \operatorname{sech} u
\end{array}\right.
$$

cujas soluções são dadas por:

$$
\cot \left(\frac{\bar{\phi}}{2}\right)=(-v+c) \operatorname{sech} u, \quad c \in \mathbb{R} .
$$


Para cada solução $\bar{\phi}$ encontrada, a superfície dada por

$$
\begin{aligned}
\bar{X} & =X+r e_{1} \\
& =X+\left(\cos \bar{\phi} \operatorname{coth} u X_{u}+\operatorname{sen} \bar{\phi} \cosh u X_{v}\right)
\end{aligned}
$$

é uma superfície de curvatura gaussiana -1. A superfície $\bar{X}$ é chamada de superfície de Kuen.
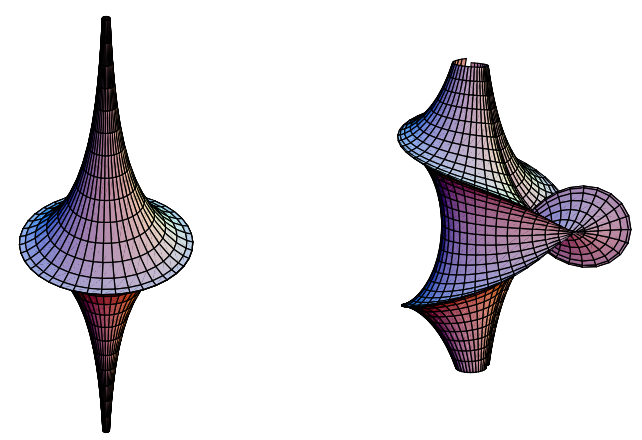

Figura 3.4: A pseudo-esfera (à esquerda) e a superfície de Kuen (à direita), obtida para $c=1$.

Observação 3.3.4. A Proposição 3.3.1 vale para qualquer solução da equação de Sine-Gordon, em particular para a solução $\phi=0$, mesmo que ela não esteja associada a nenhuma superfície. Neste caso a transformação de Bäcklund (3.29) se reduz a

$$
\left\{\begin{array}{l}
\bar{\phi}_{u}=\operatorname{sen} \bar{\phi} \csc \theta, \\
\bar{\phi}_{v}=-\operatorname{sen} \bar{\phi} \cot \theta .
\end{array}\right.
$$

Para cada $\theta \neq 0$, a solução $\bar{\phi}$ desse sistema dada por:

$$
\tan \frac{\bar{\phi}}{2}=e^{u \csc \theta-v \cot \theta+c}, \quad c \in \mathbb{R},
$$

representa uma nova solução da equação de Sine-Gordon. Assim, do Lema 2.3.5, temos

$$
\begin{aligned}
& \operatorname{sen} \bar{\phi}=\operatorname{sech}(u \csc \theta-v \cot \theta+c), \\
& \cos \bar{\phi}=-\tanh (u \csc \theta-v \cot \theta+c) .
\end{aligned}
$$

Além disso, a superfície $\bar{X}$ associada à solução $\bar{\phi}$ é determinada pelas formas quadráticas:

$$
\begin{aligned}
\overline{\mathrm{I}} & =\cos ^{2} \bar{\phi} d u^{2}+\operatorname{sen}^{2} \bar{\phi} d v^{2}, \\
\overline{\mathrm{II}} & =\operatorname{sen} \bar{\phi} \cos \bar{\phi}\left(d u^{2}-d v^{2}\right) .
\end{aligned}
$$

Em particular, tomando $c=0$ e $\theta=\frac{\pi}{2}$, obtemos

$$
\operatorname{sen} \bar{\phi}=\operatorname{sech} u, \quad \cos \bar{\phi}=-\tanh u,
$$

ou seja, $\bar{X}$ é a pseudo-esfera (veja o Exemplo 2.3.6). 
Observação 3.3.5. A transformação de Bäcklund dada pelo sistema (3.29) pode ser reescrita mais simplificadamente se considerarmos a mudança para coordenadas assintóticas:

$$
\left\{\begin{array}{l}
x=\frac{u+v}{2} \\
y=\frac{u-v}{2} .
\end{array}\right.
$$

De fato, como

$$
\left\{\begin{array}{l}
\frac{1}{2}\left(\bar{\phi}_{x}+\bar{\phi}_{y}+\phi_{x}-\phi_{y}\right)=\operatorname{sen} \bar{\phi} \cos \phi \csc \theta+\cot \theta \cos \bar{\phi} \operatorname{sen} \phi, \\
\frac{1}{2}\left(\bar{\phi}_{x}-\bar{\phi}_{y}+\phi_{x}+\phi_{y}\right)=-\cos \bar{\phi} \operatorname{sen} \phi \csc \theta-\cot \theta \operatorname{sen} \bar{\phi} \cos \phi,
\end{array}\right.
$$

somando e subtraindo estas equações, a transformação de Bäcklund se reduz a

$$
\left\{\begin{array}{l}
\bar{\phi}_{x}+\phi_{x}=\mu \operatorname{sen}(\bar{\phi}-\phi) \\
\bar{\phi}_{y}-\phi_{y}=\frac{1}{\mu} \operatorname{sen}(\bar{\phi}+\phi)
\end{array}\right.
$$

onde $\mu=\frac{1-\cos \theta}{\operatorname{sen} \theta} \neq 0$.

\subsubsection{O Teorema de Permutabilidade de Bianchi}

Considere $\bar{\phi}$ e $\overline{\bar{\phi}}$ soluções associadas a $\phi$ por $B\left(\theta_{1}\right)$ e $B\left(\theta_{2}\right)$, respectivamente. Apresentaremos, agora, um resultado do L. Bianchi que mostra que existe uma outra solução $\phi^{*} \neq \phi$ associada a $\bar{\phi}$ e $\overline{\bar{\phi}}$ por transformação de Bäcklund. Mais precisamente:

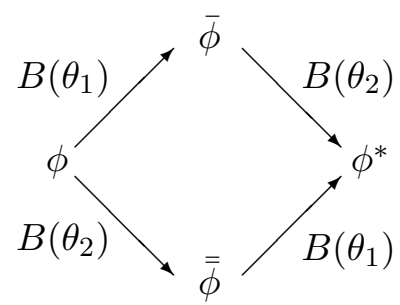

Teorema 3.3.6 (Teorema de Permutabilidade de Bianchi [7]). Sejam $\bar{\phi}$ e $\overline{\bar{\phi}}$ duas soluções da equação de Sine-Gordon que estão associadas a $\phi$ por $B\left(\theta_{1}\right)$ e $B\left(\theta_{2}\right)$, respectivamente. Então, se $\theta_{1} \neq \theta_{2}$, existe uma única solução $\phi^{*}$ associada às soluções $\bar{\phi}$ e $\overline{\bar{\phi}}$ por $B\left(\theta_{2}\right)$ e $B\left(\theta_{1}\right)$, respectivamente. Além disso, $\phi^{*}$ é determinada algebricamente por

$$
\tan \frac{\phi^{*}-\phi}{2}=\frac{\operatorname{sen} \frac{\theta_{2}+\theta_{1}}{2}}{\operatorname{sen} \frac{\theta_{2}-\theta_{1}}{2}} \tan \frac{\bar{\phi}-\overline{\bar{\phi}}}{2} .
$$

Demonstração. Por hipótese, as soluções $\bar{\phi}$ e $\overline{\bar{\phi}}$ são associadas a $\phi$ pelas transformações de Bäcklund $B\left(\theta_{1}\right)$ e $B\left(\theta_{2}\right)$, respectivamente. Da Observação 3.3.5 temos que

$$
\left\{\begin{array}{l}
\bar{\phi}_{x}+\phi_{x}=r_{1} \operatorname{sen}(\bar{\phi}-\phi), \\
\bar{\phi}_{y}-\phi_{y}=\frac{1}{r_{1}} \operatorname{sen}(\bar{\phi}+\phi),
\end{array}\right.
$$




$$
\left\{\begin{array}{l}
\overline{\bar{\phi}}_{x}+\phi_{x}=r_{2} \operatorname{sen}(\overline{\bar{\phi}}-\phi), \\
\overline{\bar{\phi}}_{y}-\phi_{y}=\frac{1}{r_{2}} \operatorname{sen}(\overline{\bar{\phi}}+\phi),
\end{array}\right.
$$

onde

$$
r_{i}=\frac{1-\cos \theta_{i}}{\operatorname{sen} \theta_{i}} \quad i=1,2
$$

Queremos obter $\phi^{*}$ que satisfaça aos sistemas:

$$
\begin{aligned}
& \left\{\begin{array}{l}
\phi_{x}^{*}+\bar{\phi}_{x}=r_{2} \operatorname{sen}\left(\phi^{*}-\bar{\phi}\right), \\
\phi_{y}^{*}-\bar{\phi}_{y}=\frac{1}{r_{2}} \operatorname{sen}\left(\phi^{*}+\bar{\phi}\right),
\end{array}\right. \\
& \left\{\begin{array}{l}
\phi_{x}^{*}+\overline{\bar{\phi}}_{x}=r_{1} \operatorname{sen}\left(\phi^{*}-\overline{\bar{\phi}}\right), \\
\phi_{y}^{*}-\overline{\bar{\phi}}_{y}=\frac{1}{r_{1}} \operatorname{sen}\left(\phi^{*}+\overline{\bar{\phi}}\right) .
\end{array}\right.
\end{aligned}
$$

Suponha que uma tal $\phi^{*}$ exista. Subtraindo as primeiras e segundas relações de (3.32) e (3.33), obtemos

$$
\begin{aligned}
& \bar{\phi}_{x}-\overline{\bar{\phi}}_{x}=r_{1} \operatorname{sen}(\bar{\phi}-\phi)-r_{2} \operatorname{sen}(\overline{\bar{\phi}}-\phi), \\
& \bar{\phi}_{y}-\overline{\bar{\phi}}_{y}=\frac{1}{r_{1}} \operatorname{sen}(\bar{\phi}+\phi)-\frac{1}{r_{2}} \operatorname{sen}(\overline{\bar{\phi}}+\phi) .
\end{aligned}
$$

Da mesma maneira, subtraindo as correspondentes de (3.34) e (3.35), temos

$$
\begin{aligned}
& \bar{\phi}_{x}-\overline{\bar{\phi}}_{x}=r_{2} \operatorname{sen}\left(\phi^{*}-\bar{\phi}\right)-r_{1} \operatorname{sen}\left(\phi^{*}-\overline{\bar{\phi}}\right), \\
& \bar{\phi}_{y}-\overline{\bar{\phi}}_{y}=-\frac{1}{r_{2}} \operatorname{sen}\left(\phi^{*}+\bar{\phi}\right)+\frac{1}{r_{1}} \operatorname{sen}\left(\phi^{*}+\overline{\bar{\phi}}\right) .
\end{aligned}
$$

Assim, de (3.36) e (3.37) resulta que

$$
\begin{aligned}
& r_{1}\left(\operatorname{sen}(\bar{\phi}-\phi)+\operatorname{sen}\left(\phi^{*}-\overline{\bar{\phi}}\right)\right)=r_{2}\left(\operatorname{sen}\left(\phi^{*}-\bar{\phi}\right)+\operatorname{sen}(\overline{\bar{\phi}}-\phi)\right), \\
& r_{1}\left(\operatorname{sen}\left(\phi^{*}+\bar{\phi}\right)-\operatorname{sen}(\overline{\bar{\phi}}+\phi)\right)=r_{2}\left(\operatorname{sen}\left(\phi^{*}+\overline{\bar{\phi}}\right)-\operatorname{sen}(\bar{\phi}+\phi)\right) .
\end{aligned}
$$

Utilizando a identidade trigonométrica:

$$
\operatorname{sen}(a \pm b) \cos (a \mp b)=\frac{1}{2}(\operatorname{sen} 2 a \pm \operatorname{sen} 2 b),
$$

temos que

$$
\begin{aligned}
& \operatorname{sen}\left(\phi^{*}-\overline{\bar{\phi}}\right)+\operatorname{sen}(\bar{\phi}-\phi)=2 \operatorname{sen}\left(\frac{\phi^{*}-\phi}{2}+\frac{\bar{\phi}-\overline{\bar{\phi}}}{2}\right) \cos \left(\frac{\phi^{*}+\phi}{2}-\frac{\bar{\phi}+\overline{\bar{\phi}}}{2}\right), \\
& \operatorname{sen}\left(\phi^{*}-\bar{\phi}\right)+\operatorname{sen}(\overline{\bar{\phi}}-\phi)=2 \operatorname{sen}\left(\frac{\phi^{*}-\phi}{2}-\frac{\bar{\phi}-\overline{\bar{\phi}}}{2}\right) \cos \left(\frac{\phi^{*}+\phi}{2}-\frac{\bar{\phi}+\overline{\bar{\phi}}}{2}\right)
\end{aligned}
$$

e, portanto, as relações (3.38) são ambas equivalentes a

$$
r_{1} \operatorname{sen}\left(\frac{\phi^{*}-\phi}{2}+\frac{\bar{\phi}-\overline{\bar{\phi}}}{2}\right)=r_{2} \operatorname{sen}\left(\frac{\phi^{*}-\phi}{2}-\frac{\bar{\phi}-\overline{\bar{\phi}}}{2}\right) .
$$

Usando a identidade trigonométrica

$$
\operatorname{sen}(\alpha \pm \beta)=\operatorname{sen} \alpha \cos \beta \pm \operatorname{sen} \beta \cos \alpha
$$


em (3.39) e substituindo $r_{1}$ e $r_{2}$ pelas respectivas expressões, obtemos

$$
\tan \frac{\phi^{*}-\phi}{2}=\frac{\operatorname{sen} \frac{\theta_{2}+\theta_{1}}{2}}{\operatorname{sen} \frac{\theta_{2}-\theta_{1}}{2}} \tan \frac{\bar{\phi}-\overline{\bar{\phi}}}{2} .
$$

Note que $\phi^{*}$ assim definida satisfaz as relações de (3.34) e (3.35) (veja [7], p. 7). Portanto, das relações acima segue que $\phi^{*}$ existe e é única.

Exemplo 3.3.7. Considere a solução $\phi=0$ da equação de Sine-Gordon. Como visto na Observação 3.3.4, para $\theta \neq 0$, obtemos uma nova solução $\bar{\phi}$ desta equação, dada por:

$$
\tan \frac{\bar{\phi}}{2}=e^{u \csc \theta-v \cot \theta+c}, \quad c \in \mathbb{R} .
$$

Em particular, para $c=0$ e escolhendo $\theta_{1}=\frac{\pi}{2}, \theta_{2}=\frac{\pi}{4}$, obtemos, respectivamente:

$$
\tan \frac{\bar{\phi}}{2}=e^{u}, \quad \tan \frac{\overline{\bar{\phi}}}{2}=e^{\sqrt{2} u-v}
$$

Então, da equação (3.31) do Teorema de Permutabilidade (conhecida como fórmula de superposição), obtemos uma quarta solução $\phi^{*}$ da equação de Sine-Gordon:

$$
\tan \frac{\phi^{*}}{2}=(\sqrt{2}+1) \frac{e^{\sqrt{2} u-v}-e^{u}}{1+e^{(\sqrt{2}+1) u-v}} .
$$

Se considerarmos a interpretação geométrica das soluções da equação de Sine-Gordon e da transformação de Bäcklund, podemos enunciar o Teorema de Permutabilidade da seguinte maneira.

Teorema 3.3.8 (Teorema de Permutabilidade). Sejam $S, \bar{S}, \overline{\bar{S}}$ superfícies parametrizadas regulares e $l_{1}: S \rightarrow \bar{S}$ e $l_{2}: S \rightarrow \overline{\bar{S}}$ congruências pseudo-esféricas com constantes $\left(\theta_{1}, r_{1}\right) e$ $\left(\theta_{2}, r_{2}\right)$, respectivamente, sendo $\theta_{i} \in(0, \pi)$ e $r_{i}>0, i=1,2$. Se $\theta_{1} \neq \theta_{2}$, então existe uma única superfície $S^{*}$ e congruências pseudo-esféricas $\bar{l}_{2}: \bar{S} \rightarrow S^{*}$ e $\bar{l}_{1}: \overline{\bar{S}} \rightarrow S^{*}$ com constantes $\left(\theta_{2}, r_{2}\right)$ $e\left(\theta_{1}, r_{1}\right)$, respectivamente, tais que

$$
\bar{l}_{2} \circ l_{1}=\bar{l}_{1} \circ l_{2}
$$

ou seja, o diagrama seguinte é comutativo:

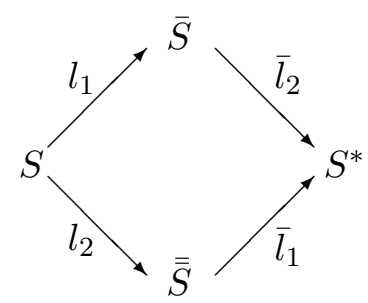

Demonstração. Sejam $X, \bar{X}, \overline{\bar{X}}: U \subset \mathbb{R}^{2} \rightarrow \mathbb{R}^{3}$ parametrizações das superfícies $S, \bar{S}$ e $\overline{\bar{S}}$, respectivamente, tais que

$$
\bar{X}=X+r_{1} e_{1}, \quad \overline{\bar{X}}=X+r_{2} \bar{e}_{1},
$$


sendo $\left\{e_{1}, e_{2}, e_{3}\right\}$ e $\left\{\bar{e}_{1}, \bar{e}_{2}, e_{3}\right\}$ triedros móveis associados à superfície $X$. Considere para $\bar{X}$ e $\overline{\bar{X}}$ os respectivos triedros móveis:

$$
\begin{aligned}
& e_{1}^{\prime}=-e_{1}, \\
& e_{2}^{\prime}=\cos \theta_{1} e_{2}+\operatorname{sen} \theta_{1} e_{3}, \\
& e_{3}^{\prime}=-\operatorname{sen} \theta_{1} e_{2}+\cos \theta_{1} e_{3}
\end{aligned}
$$

e

$$
\begin{aligned}
& e_{1}^{\prime \prime}=-\bar{e}_{1}, \\
& e_{2}^{\prime \prime}=\cos \theta_{2} \bar{e}_{2}+\operatorname{sen} \theta_{2} e_{3}, \\
& e_{3}^{\prime \prime}=-\operatorname{sen} \theta_{2} \bar{e}_{2}+\cos \theta_{2} e_{3},
\end{aligned}
$$

onde $e_{1}$ e $\bar{e}_{1}$ são campos de vetores unitários tangentes a $X$ na direção das retas de $p$ a $\bar{p}=l_{1}(p)$ e de $p$ a $\overline{\bar{p}}=l_{2}(p)$, respectivamente.

Defina as matrizes

$$
A=\left[\begin{array}{cc}
\frac{1}{\sqrt{-K}} & 0 \\
0 & 1
\end{array}\right], \quad \bar{A}=\left[\begin{array}{cc}
-\frac{1}{\sqrt{-K}} & 0 \\
0 & 1
\end{array}\right],
$$

onde $K$ é a curvatura gaussiana da superfície $X$, e

$$
D_{i}=\left[\begin{array}{cc}
\frac{1}{r_{i}} & 0 \\
0 & \cot \theta_{i}
\end{array}\right], \quad i=1,2 .
$$

Seja $C$ a matriz ortogonal tal que:

$$
\bar{e}_{i}=C_{i j} e_{j}, \quad i, j=1,2,
$$

e considere a matriz $B$ tal que

$$
B F=E,
$$

sendo

$$
E=A\left(D_{1} C-D_{2}\right), \quad F=\bar{A}\left(D_{1}-D_{2} C\right) .
$$

Note que

$$
A^{2}=\left[\begin{array}{cc}
-\frac{1}{K} & 0 \\
0 & 1
\end{array}\right] \quad \text { e } \quad D_{i}^{2}=\left[\begin{array}{cc}
\frac{1}{r_{i}^{2}} & 0 \\
0 & \cot ^{2} \theta_{i}
\end{array}\right], \quad i=1,2
$$

Portanto, como do Teorema de Bäcklund,

$$
\frac{\operatorname{sen}^{2} \theta_{1}}{r_{1}^{2}}=-K=\frac{\operatorname{sen}^{2} \theta_{2}}{r_{2}^{2}}
$$

então

$$
A^{2}\left(D_{1}^{2}-D_{2}^{2}\right)=\left(\cot ^{2} \theta_{1}-\cot ^{2} \theta_{2}\right) \text { Id }
$$

Além disso, de

$$
\begin{gathered}
E^{t} E=\left(D_{1} C-D_{2}\right)^{t} A^{2}\left(D_{1} C-D_{2}\right) \\
F^{t} F=\left(D_{1}-D_{2} C\right)^{t} \bar{A}^{2}\left(D_{1}-D_{2} C\right)=\left(D_{1}-D_{2} C\right)^{t} A^{2}\left(D_{1}-D_{2} C\right)
\end{gathered}
$$


e

$$
D_{i} A^{2} D_{j}=A^{2} D_{i} D_{j}=A^{2} D_{j} D_{i}, \quad i, j=1,2,
$$

segue que $E^{t} E=F^{t} F$, ou seja, $B$ é uma matriz ortogonal.

Definimos, então, os campos de vetores tangentes a $\bar{X}$ e $\overline{\bar{X}}$, respectivamente, por

$$
\bar{e}_{i}^{\prime}=B_{i j} e_{j}^{\prime}, \quad \bar{e}_{i}^{\prime \prime}=B_{i j} e_{j}^{\prime \prime}, \quad i, j=1,2 .
$$

Considere agora as congruências pseudo-esféricas dadas por:

$$
\begin{array}{rlrl}
\bar{l}_{2}: \bar{S} & \longrightarrow \bar{l}_{2}(\bar{S}) & \text { e } & \bar{l}_{1}: \overline{\bar{S}} \\
\bar{p} & \longmapsto \bar{p}+r_{2} \bar{e}_{1}^{\prime} & & \bar{l}_{1}(\overline{\bar{S}}) \\
& & \overline{\bar{p}} \longmapsto \overline{\bar{p}}+r_{1} \bar{e}_{1}^{\prime \prime} ;
\end{array}
$$

vamos provar que

$$
\bar{l}_{2} \circ l_{1}=\bar{l}_{1} \circ l_{2}
$$

Temos que, para todo $p \in S$,

$$
\begin{aligned}
& \left(\bar{l}_{2} \circ l_{1}\right)(p)=\bar{l}_{2}\left(p+r_{1} e_{1}\right)=p+r_{1} e_{1}+r_{2} \bar{e}_{1}^{\prime}, \\
& \left(\bar{l}_{1} \circ l_{2}\right)(p)=\bar{l}_{1}\left(p+r_{2} \bar{e}_{1}\right)=p+r_{2} \bar{e}_{1}+r_{1} \bar{e}_{1}^{\prime \prime} .
\end{aligned}
$$

Usando (3.40), (3.41), (3.42) e (3.45), obtemos

$$
\begin{aligned}
r_{1} e_{1}+r_{2} \bar{e}_{1}^{\prime}-\left(r_{2} \bar{e}_{1}+r_{1} \bar{e}_{1}^{\prime \prime}\right)= & {\left[r_{1}-\left(B_{11}+C_{11}\right) r_{2}-r_{1}\left(-B_{11} C_{11}+B_{12} C_{21} \cos \theta_{2}\right)\right] e_{1} } \\
& +\left[r_{2}\left(B_{12} \cos \theta_{1}-C_{12}\right)-r_{1}\left(-B_{11} C_{12}+B_{12} C_{22} \cos \theta_{2}\right)\right] e_{2} \\
& +B_{12}\left(r_{2} \operatorname{sen} \theta_{1}-r_{1} \operatorname{sen} \theta_{2}\right) e_{3} .
\end{aligned}
$$

Note que, de (3.43) e do Teorema de Bäcklund, resulta

$$
\begin{aligned}
& r_{1}-\left(B_{11}+C_{11}\right) r_{2}-r_{1}\left(-B_{11} C_{11}+B_{12} C_{21} \cos \theta_{2}\right)= \\
& \quad r_{1}+B_{11} C_{11} r_{1}-\left(B_{11}+C_{11}\right) r_{2}+B_{12} C_{21} \sqrt{-K} r_{1} r_{2} \cot \theta_{2}=0, \\
& r_{2}\left(B_{12} \cos \theta_{1}-C_{12}\right)-r_{1}\left(-B_{11} C_{12}+B_{12} C_{22} \cos \theta_{2}\right)= \\
& \quad C_{12}\left(B_{11} r_{1}-r_{2}\right)+\sqrt{-K} r_{1} r_{2}\left(B_{12} \cot \theta_{1}-C_{22} B_{12} \cot \theta_{2}\right)=0
\end{aligned}
$$

e, de (3.44), temos também que

$$
r_{2} \operatorname{sen} \theta_{1}-r_{1} \operatorname{sen} \theta_{2}=0
$$

de onde segue o resultado. Observe que a superfície

$$
S^{*}:=\bar{l}_{2}(\bar{S})=\bar{l}_{1}(\overline{\bar{S}}) \text {. }
$$

Vamos ilustrar o Teorema de Permutabilidade 3.3.8 em uma composição de transformações de Bäcklund aplicada à pseudo-esfera. 
Exemplo 3.3.9. Considere a parametrização da pseudo-esfera

$$
X(u, v)=(\operatorname{sech} u \cos v, \operatorname{sech} u \operatorname{sen} v, u-\tanh u)
$$

que vimos ser associada à solução $\phi$ da equação de Sine-Gordon dada por tan $\frac{\phi}{2}=e^{u}$.

Do Exemplo 3.3.3, sua transformada de Bäcklund por $\theta_{1}=\frac{\pi}{2}$ é a superfície

$$
\bar{X}=X+\cos \bar{\phi} \operatorname{coth} u X_{u}+\operatorname{sen} \bar{\phi} \cosh u X_{v}
$$

associada a $\bar{\phi}$ dada por

$$
\cot \left(\frac{\bar{\phi}}{2}\right)=(-v+c) \operatorname{sech} u
$$

Similarmente, obtemos uma outra superfície associada a $X$ integrando o sistema $B\left(\frac{\pi}{4}\right)$ :

$$
\left\{\begin{array}{l}
\overline{\bar{\phi}}_{u}=\sqrt{2} \operatorname{sen} \overline{\bar{\phi}} \tanh u+\cos \overline{\bar{\phi}} \operatorname{sech} u, \\
\overline{\bar{\phi}}_{v}=\operatorname{sech} u(1-\sqrt{2} \cos \overline{\bar{\phi}})-\operatorname{sen} \overline{\bar{\phi}} \tanh u
\end{array}\right.
$$

cuja solução é dada por

$$
\tan \left(\frac{\overline{\bar{\phi}}}{2}\right)=(\sqrt{2}+1) \frac{e^{\sqrt{2} u-v}-e^{u}}{1+e^{\sqrt{2} u-v+u}} .
$$

Esta solução está associada à superfície

$$
\overline{\bar{X}}=X+\frac{\sqrt{2}}{2}\left(\cos \overline{\bar{\phi}} \operatorname{coth} u X_{u}+\operatorname{sen} \overline{\bar{\phi}} \cosh u X_{v}\right) .
$$

Segue da prova do Teorema de Permutabilidade, que a matriz $C$, definida por (3.42), é

$$
C=\left[\begin{array}{cc}
\cos (\bar{\phi}-\overline{\bar{\phi}}) & -\operatorname{sen}(\bar{\phi}-\overline{\bar{\phi}}) \\
\operatorname{sen}(\bar{\phi}-\overline{\bar{\phi}}) & \cos (\bar{\phi}-\overline{\bar{\phi}})
\end{array}\right]
$$

Calculando a matriz $B$, definida por (3.43), concluímos que a quarta superfície $X^{*}$, associada a $\bar{X}$ por $B\left(\theta_{2}\right)$ e a $\overline{\bar{X}}$ por $B\left(\theta_{1}\right)$, é dada explicitamente por:

$$
X^{*}=\bar{X}+r_{2} \bar{e}_{1}^{\prime}=\bar{X}+\frac{\sqrt{2}}{2}\left(-B_{11} e_{1}+B_{12} e_{3}\right),
$$

onde $e_{3}$ é o campo de vetores normal à superfície $X, e_{1}=\cos \phi_{2} \tilde{e}_{1}+\operatorname{sen} \phi_{2} \tilde{e}_{2} \mathrm{e}$

$$
\tilde{e}_{1}=\sec \phi X_{u}, \quad \tilde{e}_{2}=\csc \phi X_{v} .
$$




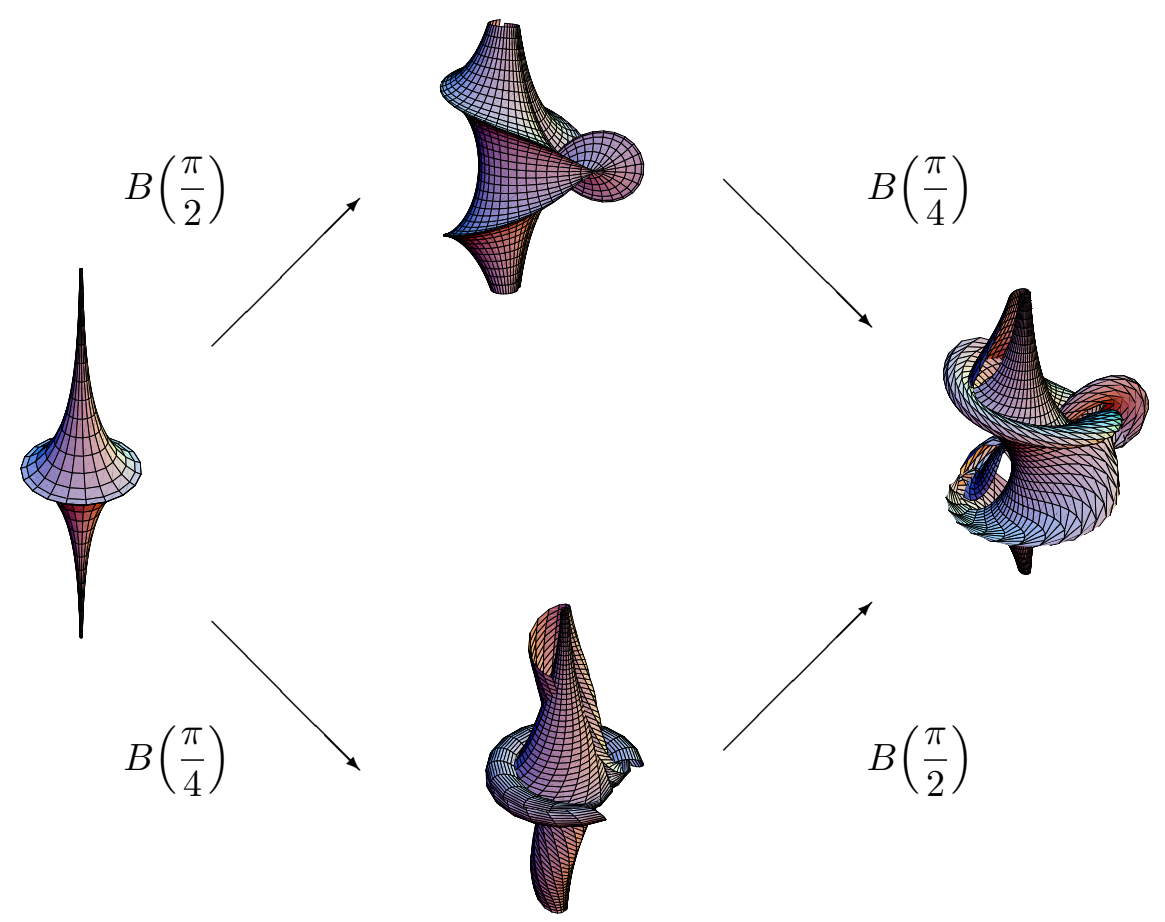

Figura 3.5: Diagrama de permutabilidade de Bianchi para a pseudo-esfera.

Observação 3.3.10. Na Proposição 3.3 .1 vimos que, dada uma solução real $\phi$ da equação de Sine-Gordon, obtemos uma outra solução real desta equação integrando a transformação de Bäcklund $B(\theta), \theta \in \mathbb{R}$. Considerando, agora, um número complexo $z_{1}$, a solução $\phi_{1}$ obtida integrando $B\left(z_{1}\right)$ assume valores complexos. Mais ainda, se $z_{2}=\bar{z}_{1}$ e $\phi_{2}$ é obtida integrando $B\left(z_{2}\right)$ :

$$
\left\{\begin{array}{l}
\phi_{2 u}+\phi_{v}=\operatorname{sen} \phi_{2} \cos \phi \csc z_{2}+\cot z_{2} \cos \phi_{2} \operatorname{sen} \phi, \\
\phi_{2 v}+\phi_{u}=-\cos \phi_{2} \operatorname{sen} \phi \csc z_{2}-\cot z_{2} \operatorname{sen} \phi_{2} \cos \phi,
\end{array}\right.
$$

então $\phi_{2}=\bar{\phi}_{1}$. De fato, este resultado segue conjugando o sistema $B\left(z_{1}\right)$ :

$$
\left\{\begin{array}{l}
\bar{\phi}_{1 u}+\phi_{v}=\operatorname{sen} \bar{\phi}_{1} \cos \phi \csc \bar{z}_{1}+\cot \bar{z}_{1} \cos \bar{\phi}_{1} \operatorname{sen} \phi \\
\bar{\phi}_{1 v}+\phi_{u}=-\cos \bar{\phi}_{1} \operatorname{sen} \phi \csc \bar{z}_{1}-\cot \bar{z}_{1} \operatorname{sen} \bar{\phi}_{1} \cos \phi
\end{array}\right.
$$

Observe que a solução $\phi^{*}$ obtida por (3.31) é real, pois:

$$
\begin{aligned}
\tan \frac{\phi^{*}-\phi}{2} & =\frac{\operatorname{sen} \frac{z_{2}+z_{1}}{2}}{\operatorname{sen} \frac{z_{2}-z_{1}}{2}} \tan \frac{\phi_{1}-\phi_{2}}{2} \\
& =-\frac{\operatorname{sen}\left(\mathcal{R} e z_{1}\right)}{\operatorname{senh}\left(\mathcal{I} m z_{1}\right)} \tanh \left(\mathcal{I} m \phi_{1}\right)
\end{aligned}
$$

onde foi usado que $\operatorname{sen}(i y)=i \operatorname{senh}(y)$ e $\cos (i y)=\cosh y$. 


\subsection{Transformação de Bäcklund para a equação de Sine-Gordon elíptica}

No Capítulo 3 vimos que as superfícies parametrizadas regulares de curvatura gaussiana constante positiva estão associadas às soluções da equação de Sine-Gordon elíptica

$$
\Delta \phi=-\operatorname{senh} \phi \cosh \phi .
$$

Nesta seção, vamos apresentar a transformação de Bäcklund para esta equação e obter o correspondente do Teorema de Permutabilidade 3.3.6.

Teorema 3.4.1. Seja $\phi(u, v)$ uma solução da equação de Sine-Gordon elíptica e $\theta \in \mathbb{C}$. Então a transformação de Bäcklund

$$
\left\{\begin{array}{l}
\phi_{u}^{*}+i \phi_{v}=\operatorname{senh} \theta \cosh \phi \operatorname{senh} \phi^{*}+\cosh \theta \operatorname{senh} \phi \cosh \phi^{*}, \\
i \phi_{v}^{*}+\phi_{u}=-\operatorname{senh} \theta \operatorname{senh} \phi \cosh \phi^{*}-\cosh \theta \cosh \phi \operatorname{senh} \phi^{*},
\end{array}\right.
$$

é integrável. Mais ainda, $\phi^{*}$ é uma outra solução de (3.48).

Demonstração. Para mostrar que a solução $\phi^{*}$ existe, isto é, que (3.49) é integrável, vamos verificar que $\phi_{u v}^{*}=\phi_{v u}^{*}$. Assim mostrado, segue do Teorema de Frobenius que, existe uma única solução $\phi^{*}$, num domínio simplesmente conexo, que satisfaz a condição inicial $\phi^{*}\left(u_{0}, v_{0}\right)$.

De fato, derivando a primeira equação do sistema (3.49) com relação a $v$ e a segunda com relação a $u$, obtemos, respectivamente,

$$
\begin{aligned}
\phi_{u v}^{*}+i \phi_{v v}= & {\left[\operatorname{senh} \theta \operatorname{senh} \phi \operatorname{senh} \phi^{*}+\cosh \theta \cosh \phi \cosh \phi^{*}\right] \phi_{v} } \\
& +\left[\operatorname{senh} \theta \cosh \phi \cosh \phi^{*}+\cosh \theta \operatorname{senh} \phi \operatorname{senh} \phi^{*}\right] \phi_{v}^{*}
\end{aligned}
$$

$\mathrm{e}$

$$
\begin{aligned}
i \phi_{v u}^{*}+\phi_{u u}= & -\left[\operatorname{senh} \theta \cosh \phi \cosh \phi^{*}+\cosh \theta \operatorname{senh} \phi \operatorname{senh} \phi^{*}\right] \phi_{u} \\
& -\left[\operatorname{senh} \theta \operatorname{senh} \phi \operatorname{senh} \phi^{*}+\cosh \theta \cosh \phi \cosh \phi^{*}\right] \phi_{u}^{*}
\end{aligned}
$$

Somando a primeira relação com a segunda multiplicada pelo imaginário puro $i$, temos

$$
\begin{aligned}
\phi_{u v}^{*}-\phi_{v u}^{*}+i \Delta \phi= & -i\left[\operatorname{senh} \theta \operatorname{senh} \phi \operatorname{senh} \phi^{*}+\cosh \theta \cosh \phi \cosh \phi^{*}\right]\left(\phi_{u}^{*}+i \phi_{v}\right) \\
& -i\left[\operatorname{senh} \theta \cosh \phi \cosh \phi^{*}+\cosh \theta \operatorname{senh} \phi \operatorname{senh} \phi^{*}\right]\left(i \phi_{v}^{*}+\phi_{u}\right) \\
= & -i \operatorname{senh} \phi \cosh \phi .
\end{aligned}
$$

Como $\phi$ é solução de (3.48), segue que $\phi_{u v}^{*}=\phi_{v u}^{*}$, isto é, fixada uma condição inicial $\phi^{*}\left(u_{0}, v_{0}\right)$, a solução $\phi^{*}$ existe e é única.

Para mostrar que $\phi^{*}$ é também solução da equação (3.48), basta derivar a primeira equação de (3.49) com relação a $u$ e a segunda com relação a $v$, obtendo

$$
\begin{aligned}
\phi_{u u}^{*}+i \phi_{v u}= & {\left[\operatorname{senh} \theta \operatorname{senh} \phi \operatorname{senh} \phi^{*}+\cosh \theta \cosh \phi \cosh \phi^{*}\right] \phi_{u} } \\
& +\left[\operatorname{senh} \theta \cosh \phi \cosh \phi^{*}+\cosh \theta \operatorname{senh} \phi \operatorname{senh} \phi^{*}\right] \phi_{u}^{*}, \\
i \phi_{v v}^{*}+\phi_{u v}= & -\left[\operatorname{senh} \theta \cosh \phi \cosh \phi^{*}+\cosh \theta \operatorname{senh} \phi \operatorname{senh} \phi^{*}\right] \phi_{v} \\
& -\left[\operatorname{senh} \theta \operatorname{senh} \phi \operatorname{senh} \phi^{*}+\cosh \theta \cosh \phi \cosh \phi^{*}\right] \phi_{v}^{*} .
\end{aligned}
$$


Assim, somando a primeira equação com a segunda multiplicada por $(-i)$, obtemos

$$
\phi_{u u}^{*}+\phi_{v v}^{*}+i\left[\phi_{v u}-\phi_{u v}\right]=-\operatorname{senh} \phi^{*} \cosh \phi^{*}
$$

isto é,

$$
\Delta \phi^{*}=-\operatorname{senh} \phi^{*} \cosh \phi^{*}
$$

Denotaremos por $\tilde{B}(\theta)$ a transformação de Bäcklund dada pelo sistema (3.49) e denominaremos a solução $\phi^{*}$, obtida a partir de $\phi$, de solução associada a $\phi$ por $\tilde{B}(\theta)$.

Provaremos uma fórmula de superposição para a equação de Sine-Gordon elíptica.

Teorema 3.4.2 (Teorema de Permutabilidade). Sejam $\phi_{1}$ e $\phi_{2}$ duas soluções da equação de Sine-Gordon elíptica associadas a $\phi$ por $\tilde{B}\left(\theta_{1}\right)$ e $\tilde{B}\left(\theta_{2}\right)$, respectivamente. Se $\theta_{1} \neq \theta_{2}$, então existe uma única solução $\phi^{*}$ associada a $\phi_{1}$ e $\phi_{2}$ por $\tilde{B}\left(\theta_{2}\right)$ e $\tilde{B}\left(\theta_{1}\right)$, respectivamente. Além disso, $\phi^{*}$ é determinada por:

$$
\tanh \frac{\phi^{*}-\phi}{2}=\operatorname{coth} \frac{\theta_{1}-\theta_{2}}{2} \tanh \frac{\phi_{1}-\phi_{2}}{2} .
$$

Demonstração. Como, $\phi_{1}$ e $\phi_{2}$ são soluções associadas a $\phi$ por $\tilde{B}\left(\theta_{1}\right)$ e $\tilde{B}\left(\theta_{2}\right)$, respectivamente, temos

$$
\left\{\begin{array}{l}
\phi_{1 u}+i \phi_{v}=\operatorname{senh} \theta_{1} \cosh \phi \operatorname{senh} \phi_{1}+\cosh \theta_{1} \operatorname{senh} \phi \cosh \phi_{1} \\
i \phi_{1 v}+\phi_{u}=-\operatorname{senh} \theta_{1} \operatorname{senh} \phi \cosh \phi_{1}-\cosh \theta_{1} \cosh \phi \operatorname{senh} \phi_{1}
\end{array}\right.
$$

$\mathrm{e}$

$$
\left\{\begin{array}{l}
\phi_{2 u}+i \phi_{v}=\operatorname{senh} \theta_{2} \cosh \phi \operatorname{senh} \phi_{2}+\cosh \theta_{2} \operatorname{senh} \phi \cosh \phi_{2}, \\
i \phi_{2 v}+\phi_{u}=-\operatorname{senh} \theta_{2} \operatorname{senh} \phi \cosh \phi_{2}-\cosh \theta_{2} \cosh \phi \operatorname{senh} \phi_{2} .
\end{array}\right.
$$

Queremos encontrar $\phi^{*}$ satisfazendo

$$
\left\{\begin{array}{l}
\phi_{u}^{*}+i \phi_{1 v}=\operatorname{senh} \theta_{2} \cosh \phi_{1} \operatorname{senh} \phi^{*}+\cosh \theta_{2} \operatorname{senh} \phi_{1} \cosh \phi^{*}, \\
i \phi_{v}^{*}+\phi_{1 u}=-\operatorname{senh} \theta_{2} \operatorname{senh} \phi_{1} \cosh \phi^{*}-\cosh \theta_{2} \cosh \phi_{1} \operatorname{senh} \phi^{*}
\end{array}\right.
$$

e

$$
\left\{\begin{array}{l}
\phi_{u}^{*}+i \phi_{2 v}=\operatorname{senh} \theta_{1} \cosh \phi_{2} \operatorname{senh} \phi^{*}+\cosh \theta_{1} \operatorname{senh} \phi_{2} \cosh \phi^{*} \\
i \phi_{v}^{*}+\phi_{2 u}=-\operatorname{senh} \theta_{1} \operatorname{senh} \phi_{2} \cosh \phi^{*}-\cosh \theta_{1} \cosh \phi_{2} \operatorname{senh} \phi^{*}
\end{array}\right.
$$

Suponha que $\phi^{*}$ exista. Assim, subtraindo as equações correspondentes de (3.51) e (3.52) temos

$$
\begin{aligned}
\phi_{1 u}-\phi_{2 u}= & \operatorname{senh} \theta_{1} \cosh \phi \operatorname{senh} \phi_{1}+\cosh \theta_{1} \operatorname{senh} \phi \cosh \phi_{1} \\
& -\operatorname{senh} \theta_{2} \cosh \phi \operatorname{senh} \phi_{2}-\cosh \theta_{2} \operatorname{senh} \phi \cosh \phi_{2}, \\
i \phi_{1 v}-i \phi_{2 v}= & -\operatorname{senh} \theta_{1} \operatorname{senh} \phi \cosh \phi_{1}-\cosh \theta_{1} \cosh \phi \operatorname{senh} \phi_{1} \\
& +\operatorname{senh} \theta_{2} \operatorname{senh} \phi \cosh \phi_{2}+\cosh \theta_{2} \cosh \phi \operatorname{senh} \phi_{2} .
\end{aligned}
$$

Analogamente, subtraindo as equações correspondentes de (3.53) e (3.54), temos

$$
\begin{aligned}
i \phi_{1 v}-i \phi_{2 v}= & \operatorname{senh} \theta_{2} \cosh \phi_{1} \operatorname{senh} \phi^{*}+\cosh \theta_{2} \cosh \phi_{1} \cosh \phi^{*} \\
& -\operatorname{senh} \theta_{1} \cosh \phi_{2} \operatorname{senh} \phi^{*}-\cosh \theta_{1} \operatorname{senh} \phi_{2} \cosh \phi^{*}, \\
\phi_{1 u}-\phi_{2 u}= & -\operatorname{senh} \theta_{2} \operatorname{senh} \phi_{1} \cosh \phi^{*}-\cosh \theta_{2} \cosh \phi_{1} \operatorname{senh} \phi^{*} \\
& +\operatorname{senh} \theta_{1} \operatorname{senh} \phi_{2} \cosh \phi^{*}+\cosh \theta_{1} \cosh \phi_{2} \operatorname{senh} \phi^{*} .
\end{aligned}
$$


Igualando as expressões correspondentes, obtemos

$$
\begin{aligned}
& \operatorname{senh} \theta_{1}\left[\operatorname{senh} \phi_{1} \cosh \phi-\operatorname{senh} \phi_{2} \cosh \phi^{*}\right] \\
& +\cosh \theta_{1}\left[\operatorname{senh} \phi \cosh \phi_{1}-\operatorname{senh} \phi^{*} \cosh \phi_{2}\right] \\
& +\operatorname{senh} \theta_{2}\left[\operatorname{senh} \phi_{1} \cosh \phi^{*}-\operatorname{senh} \phi_{2} \cosh \phi\right] \\
& +\cosh \theta_{2}\left[\operatorname{senh} \phi^{*} \cosh \phi_{1}-\operatorname{senh} \phi \cosh \phi_{2}\right]=0
\end{aligned}
$$

e

$$
\begin{aligned}
& \operatorname{senh} \theta_{1}\left[\operatorname{senh} \phi \cosh \phi_{1}-\operatorname{senh} \phi^{*} \cosh \phi_{2}\right] \\
& +\cosh \theta_{1}\left[\operatorname{senh} \phi_{1} \cosh \phi-\operatorname{senh} \phi_{2} \cosh \phi^{*}\right] \\
& +\operatorname{senh} \theta_{2}\left[\operatorname{senh} \phi^{*} \cosh \phi_{1}-\operatorname{senh} \phi \cosh \phi_{2}\right] \\
& +\cosh \theta_{2}\left[\operatorname{senh} \phi_{1} \cosh \phi^{*}-\operatorname{senh} \phi_{2} \cosh \phi\right]=0 .
\end{aligned}
$$

Utilizando a identidade

$$
\operatorname{senh} a \cosh b-\operatorname{senh} c \cosh d=\tilde{L}\left(\tanh \frac{a-c}{2}+\tanh \frac{b-d}{2} \tanh \frac{b+d}{2} \tanh \frac{a+c}{2}\right),
$$

onde

$$
\tilde{L}=\frac{\left(1-\tanh ^{2} \frac{a}{2} \tanh ^{2} \frac{c}{2}\right)\left(1-\tanh ^{2} \frac{d}{2} \tanh ^{2} \frac{b}{2}\right)}{\left(1-\tanh ^{2} \frac{a}{2}\right)\left(1-\tanh ^{2} \frac{b}{2}\right)\left(1-\tanh ^{2} \frac{c}{2}\right)\left(1-\tanh ^{2} \frac{d}{2}\right)},
$$

obtemos, de (3.55), que

$$
\begin{aligned}
& \tanh \frac{\phi_{1}-\phi_{2}}{2}\left[\operatorname{senh} \theta_{1}+\operatorname{senh} \theta_{2}\right]+\tanh \frac{\phi-\phi^{*}}{2}\left[\cosh \theta_{1}-\cosh \theta_{2}\right] \\
& +\tanh \frac{\phi-\phi^{*}}{2} \tanh \frac{\phi+\phi^{*}}{2} \tanh \frac{\phi_{1}+\phi_{2}}{2}\left[\operatorname{senh} \theta_{1}-\operatorname{senh} \theta_{2}\right] \\
& +\tanh \frac{\phi_{1}-\phi_{2}}{2} \tanh \frac{\phi_{1}+\phi_{2}}{2} \tanh \frac{\phi+\phi^{*}}{2}\left[\cosh \theta_{1}+\cosh \theta_{2}\right]=0 .
\end{aligned}
$$

Conseqüentemente, como

$$
\begin{aligned}
& \cosh a+\cosh b=2 \cosh \frac{a-b}{2} \cosh \frac{a+b}{2} \\
& \cosh a-\cosh b=2 \operatorname{senh} \frac{a-b}{2} \operatorname{senh} \frac{a+b}{2} \\
& \operatorname{senh} a \pm \operatorname{senh} b=2 \operatorname{senh} \frac{a \pm b}{2} \cosh \frac{a \mp b}{2}
\end{aligned}
$$

segue que, ou vale $(3.50)$ ou

$$
\tanh \frac{\phi^{*}+\phi}{2}=-\tanh \frac{\theta_{1}+\theta_{2}}{2} \operatorname{coth} \frac{\phi_{1}+\phi_{2}}{2} .
$$

Procedendo da mesma maneira com a relação (3.56), obtemos que ou vale a equação (3.50) ou

$$
\tanh \frac{\phi^{*}+\phi}{2}=-\operatorname{coth} \frac{\theta_{1}+\theta_{2}}{2} \operatorname{coth} \frac{\phi_{1}+\phi_{2}}{2} .
$$

Observe que, supondo que não vale a equação (3.50), então de (3.57) e (3.58) temos que $\tanh \frac{\theta_{1}+\theta_{2}}{2}= \pm 1$, o que é absurdo. Assim, segue que $\phi^{*}$ existe e é determinada algebricamente por $(3.50)$. 
Do Teorema de Permutabilidade segue que, a partir de uma solução real da equação de Sine-Gordon elíptica, obtemos uma família de soluções reais da mesma equação, como veremos no seguinte:

Corolário 3.4.3. Sejam $\phi$ e $\phi_{1}$ duas soluções da equação de Sine-Gordon elíptica (3.48) tal que $\phi_{1}$ é associada a $\phi$ por $\tilde{B}\left(\theta_{1}\right)$, onde $\theta_{1} \in \mathbb{C}$. Então, se $\phi$ é uma função a valores reais, a função $\phi^{*}$ definida por

$$
\tanh \frac{\phi^{*}-\phi}{2}=\tanh \left(\mathcal{R} e \theta_{1}\right) \tanh \left(\mathcal{R} e \phi_{1}\right)
$$

é uma outra solução real de (3.48).

Demonstração. Por hipótese, $\phi_{1}$ é associada a $\phi$ por $\tilde{B}\left(\theta_{1}\right)$. Tomando $\phi_{2}=-\bar{\phi}_{1}$ e $\theta_{2}=\pi i-\bar{\theta}_{1}$, vamos mostrar que $\phi_{2}$ está associada a $\phi$ por $\tilde{B}\left(\theta_{2}\right)$, isto é,

$$
\left\{\begin{array}{l}
\phi_{2 u}+i \phi_{v}=\operatorname{senh} \theta_{2} \cosh \phi \operatorname{senh} \phi_{2}+\cosh \theta_{2} \operatorname{senh} \phi \cosh \phi_{2} \\
i \phi_{2 v}+\phi_{u}=-\operatorname{senh} \theta_{2} \operatorname{senh} \phi \cosh \phi_{2}-\cosh \theta_{2} \cosh \phi \operatorname{senh} \phi_{2}
\end{array}\right.
$$

Provaremos somente a primeira equação deste sistema, pois a segunda segue de forma análoga.

Note que a equação referida equivale a

$$
-\bar{\phi}_{1 u}+i \phi_{v}=-\operatorname{senh}\left(\pi i-\bar{\theta}_{1}\right) \cosh \phi \operatorname{senh}\left(-\bar{\phi}_{1}\right)-\cosh \left(\pi i-\bar{\theta}_{1}\right) \operatorname{senh} \phi \cosh \left(-\bar{\phi}_{1}\right),
$$

isto é,

$$
-\bar{\phi}_{1 u}+i \phi_{v}=-\overline{\operatorname{senh} \theta_{1}} \cosh \phi \overline{\operatorname{senh} \phi_{1}}-\overline{\cosh \theta_{1}} \operatorname{senh} \phi \overline{\cosh \phi_{1}},
$$

de onde, por conjugação, resulta que

$$
\phi_{1 u}+i \phi_{v}=\operatorname{senh} \theta_{1} \cosh \phi \operatorname{senh} \phi_{1}+\cosh \theta_{1} \operatorname{senh} \phi \cosh \phi_{1},
$$

que vale, por hipótese. Assim, do Teorema de Permutabilidade, a solução $\phi^{*}$ associada a $\phi_{1}$ e $\phi_{2}$ por $\tilde{B}\left(\theta_{2}\right)$ e $\tilde{B}\left(\theta_{1}\right)$, respectivamente, dada por

$$
\begin{aligned}
\tanh \frac{\phi^{*}-\phi}{2} & =\operatorname{coth}\left(\mathcal{R} e \theta_{1}-\frac{\pi}{2} i\right) \tanh \left(\mathcal{R} e \phi_{1}\right) \\
& =\tanh \left(\mathcal{R} e \theta_{1}\right) \tanh \left(\mathcal{R} e \phi_{1}\right)
\end{aligned}
$$

é uma outra solução de (3.48).

Observe que, como a expressão da direita é menor que 1 e real, esta equação é compatível e a função $\phi^{*}$ é a valores reais.

Decorre deste corolário que para cada solução real da equação de Sine-Gordon elíptica, obtemos uma família a 4 parâmetros de soluções reais da mesma equação. Estes parâmetros provem das partes reais e imaginárias de $\theta_{1}$ e da condição inicial $\phi_{1}\left(u_{0}, v_{0}\right)$.

Aplicaremos este resultado no seguinte

Exemplo 3.4.4. Considere a solução $\phi \equiv 0$ da equação de Sine-Gordon elíptica. Do Teorema 3.4.1, para cada $\theta_{1} \in \mathbb{C}$, existe uma família de soluções $\phi_{1}$ satisfazendo a transformação de Bäcklund

$$
\left\{\begin{array}{l}
\phi_{1 u}=\operatorname{senh} \theta_{1} \operatorname{senh} \phi_{1} \\
\phi_{1 v}=i \cosh \theta_{1} \operatorname{senh} \phi_{1}
\end{array}\right.
$$


dada por:

$$
\tanh \frac{\phi_{1}}{2}=e^{u \operatorname{senh} \theta_{1}+i v \cosh \theta_{1}+c},
$$

onde a constante $c$ depende da condição inicial $\phi_{1}\left(u_{0}, v_{0}\right)$. Considerando $c=0$ e $\theta_{1} \in \mathbb{R}$, vamos aplicar o Corolário 3.4.3 para obter uma família de soluções reais da equação de Sine-Gordon elíptica. De fato, a nova solução $\phi^{*}$ de (3.48) é dada por

$$
\tanh \frac{\phi^{*}}{2}=\tanh \theta_{1} \tanh \left(\mathcal{R} e \phi_{1}\right)
$$

Segue de (3.60) que

$$
e^{2\left(u \operatorname{senh} \theta_{1}+i v \cosh \theta_{1}\right)}=\tanh ^{2} \frac{\phi_{1}}{2}=\frac{\cosh \phi_{1}-1}{\cosh \phi_{1}+1},
$$

isto é,

$$
\cosh \phi_{1}=-\operatorname{coth}\left(u \operatorname{senh} \theta_{1}+i v \cosh \theta_{1}\right)
$$

e, portanto,

$$
\operatorname{senh} \phi_{1}=-\operatorname{cossech}\left(u \operatorname{senh} \theta_{1}+i v \cosh \theta_{1}\right) .
$$

Separando as partes real e imaginária de (3.61) e (3.62), segue que

$$
\tanh \left(\mathcal{R} e \phi_{1}\right)=\frac{\cos \left(v \cosh \theta_{1}\right)}{\cosh \left(u \operatorname{senh} \theta_{1}\right)}
$$

de onde obtemos

$$
\tanh \frac{\phi^{*}}{2}=\tanh \theta_{1} \frac{\cos \left(v \cosh \theta_{1}\right)}{\cosh \left(u \operatorname{senh} \theta_{1}\right)}
$$




\section{Capítulo 4}

\section{Congruências de Guichard}

Dedicaremos este capítulo ao estudo de dois importantes teoremas de C. Guichard relacionados à teoria das deformações das congruências de retas normais. Estes teoremas permitirão obter pares de superfícies de mesma curvatura média constante ou de mesma curvatura gaussiana constante não nula, a partir de superfícies isométricas a certas superfícies de revolução. Tais teoremas, como o mesmo L. Bianchi ressalta em [6], foram apresentadas em 1899 pelo autor no trabalho [17], porém sem as demonstrações. A partir deles, Bianchi deduz novas transformações de superfícies de curvatura constante cujo assunto é o principal objeto de estudo do artigo [6].

Considere uma congruência de retas normal a uma superfície $S$, ou seja, suponha que seja dada uma superfície $S_{0}$, flexível e inextensível, de pontos $p_{0}$ dos quais saem segmentos de reta $p_{0} p$ normais em $p$ a $S$. Se podemos deformar a superfície $S_{0}$ de modo que os segmentos $p_{0} p$ acompanhem a superfície mantendo-se ligados a ela nos extremos $p_{0}$, diremos que deformamos a congruência dada. Veremos que o Teorema de Beltrami garante que para todas as deformações $\bar{S}_{0}$, de $S_{0}$, o lugar $\bar{S}$ que os extremos $p$ descrevem (neste processo de deformação) é sempre uma superfície ortogonal aos segmentos $p_{0} p$, ou seja, que se obtém uma congruência de retas normal a $\bar{S}$.

Suponha, então, que para uma dada superfície $S_{0}$, a superfície $S$ seja de Weingarten, isto é, suas curvaturas principais $\kappa_{1}, \kappa_{2}$ são ligadas por uma relação:

$$
\psi\left(\kappa_{1}, \kappa_{2}\right)=0
$$

Nos propomos tratar do seguinte problema que foi proposto por Bianchi e por ele chamado de "Problema A" em [6] (p. 186).

Problema 4.0.5. Quando acontece que, para toda deformação (isométrica) de $S_{0}$, as curvaturas principais da superfície $\bar{S}$ estão sempre ligadas pela mesma relação $\psi\left(\bar{\kappa}_{1}, \bar{\kappa}_{2}\right)=0$ ?

Veremos que o Teorema de Weingarten fornece uma primeira resposta a este problema para o caso em que $S_{0}$ é uma superfície de revolução e os segmentos de reta $p_{0} p$ são tangentes a $S_{0}$ (na direção dos meridianos) e normais a $S$.

Para iniciarmos o estudo deste problema, vamos formalizar a definição de congruência de retas normal a uma dada superfície. 


\subsection{Congruência de retas normal}

Definição 4.1.1. Seja $X_{0}: U \subset \mathbb{R}^{2} \rightarrow \mathbb{R}^{3}$ uma superfície parametrizada regular e considere $X: U \subset \mathbb{R}^{2} \rightarrow \mathbb{R}^{3}$ uma aplicação diferenciável definida por:

$$
X(q)=X_{0}(q)+T(q) \xi(q), \quad q \in U,
$$

onde:

(i) $T: U \rightarrow \mathbb{R}-\{0\}$ é uma aplicação diferenciável;

(ii) $\xi: U \rightarrow \mathbb{R}^{3}$ é um campo diferenciável de vetores unitários tal que $\langle d X, \xi\rangle=0$ (isto é, $\forall q \in U$ e $\left.v \in \mathbb{R}^{2},\left\langle d X_{q}(v), \xi(q)\right\rangle=0\right)$.

Então a aplicação

$$
\begin{aligned}
l: X_{0}(U) & \longrightarrow X(U) \\
X_{0}(q) & \longmapsto X(q)
\end{aligned}
$$

é chamada de congruência de retas normal a $X$.

Note que, se $X$ for uma superfície parametrizada regular, da condição (ii) resulta que, para cada $q \in U$, a reta determinada pelos pontos $X_{0}(q)$ e $X(q)$ é normal a $X$.

Exemplo 4.1.2. Se $X$ e $X_{0}$ forem superfícies paralelas à distância $a \neq 0$, isto é,

$$
X(u, v)=X_{0}(u, v)+a e_{3}^{0}(u, v),
$$

onde $e_{3}^{0}$ é um campo de vetores unitários normal a $X_{0}$, então temos uma congruência de retas normal a ambas as superfícies.

Considere, agora, $X_{0}: U \subset \mathbb{R}^{2} \rightarrow \mathbb{R}^{3}$ uma superfície parametrizada regular, $e_{3}^{0}$ um campo unitário normal a $X_{0}$ e uma congruência de retas dada por:

$$
X=X_{0}+T \xi, \quad \xi \neq \pm e_{3}^{0} .
$$

Definindo $e_{1}^{0}$ como sendo o campo unitário na direção da projeção tangente de $\xi$ em $X_{0}$, então,

$$
X=X_{0}+T\left(\cos \theta e_{1}^{0}+\operatorname{sen} \theta e_{3}^{0}\right),
$$

onde $\theta$ é o ângulo formado entre $\xi$ e $e_{1}^{0}$. A partir dos campos $e_{1}^{0}$ e $e_{3}^{0}$ determinamos um triedro móvel $\left\{e_{1}^{0}, e_{2}^{0}, e_{3}^{0}\right\}$ associado a $X_{0}$ e denotamos por $\omega_{1}^{0}, \omega_{2}^{0}, \omega_{i j}^{0}, i, j=1,2,3$, as 1 -formas associadas a este triedro móvel. Ressaltamos que estas notações serão usadas no restante do capítulo.

A seguir apresentaremos a condição necessária e suficiente para que uma congruência de retas seja normal à aplicação $X$. Este resultado será muito utilizado no decorrer deste capítulo.

Lema 4.1.3. Uma congruência de retas

$$
X=X_{0}+T\left(\cos \theta e_{1}^{0}+\operatorname{sen} \theta e_{3}^{0}\right)
$$

é normal a $X$ se, e somente se,

$$
\cos \theta \omega_{1}^{0}+d T=0
$$


Neste caso, temos

$$
\begin{aligned}
d X= & \left(\operatorname{sen} \theta \omega_{1}^{0}-T d \theta-T \omega_{13}^{0}\right)\left(\operatorname{sen} \theta e_{1}^{0}-\cos \theta e_{3}^{0}\right) \\
& +\left(\omega_{2}^{0}+T \cos \theta \omega_{12}^{0}-T \operatorname{sen} \theta \omega_{23}^{0}\right) e_{2}^{0}
\end{aligned}
$$

$e$

$$
-\operatorname{sen} \theta d \theta \wedge \omega_{1}^{0}+\cos \theta d \omega_{1}^{0}=0
$$

Demonstração. Por definição, (4.1) é uma congruência de retas normal a $X$ se, e só se,

$$
\left\langle d X, \cos \theta e_{1}^{0}+\operatorname{sen} \theta e_{3}^{0}\right\rangle=0 .
$$

Assim, como

$$
\begin{aligned}
d X= & d X_{0}+d\left[T\left(\cos \theta e_{1}^{0}+\operatorname{sen} \theta e_{3}^{0}\right)\right] \\
= & \left(\omega_{1}^{0}+d T \cos \theta-T \operatorname{sen} \theta d \theta-T \operatorname{sen} \theta \omega_{13}^{0}\right) e_{1}^{0} \\
& +\left(\omega_{2}^{0}+T \cos \theta \omega_{12}^{0}-T \operatorname{sen} \theta \omega_{23}^{0}\right) e_{2}^{0} \\
& +\left(d T \operatorname{sen} \theta+T \cos \theta \omega_{13}^{0}+T \cos \theta d \theta\right) e_{3}^{0},
\end{aligned}
$$

segue que

$$
\begin{aligned}
0= & \left(\omega_{1}^{0}+d T \cos \theta-T \operatorname{sen} \theta d \theta-T \operatorname{sen} \theta \omega_{13}^{0}\right) \cos \theta \\
& +\left(d T \operatorname{sen} \theta+T \cos \theta \omega_{13}^{0}+T \cos \theta d \theta\right) \operatorname{sen} \theta \\
= & \cos \theta \omega_{1}^{0}+d T .
\end{aligned}
$$

Substituindo $d T=-\cos \theta \omega_{1}^{0}$ em (4.5) temos (4.3); a (4.4) se obtém derivando (4.2).

Observação 4.1.4. No caso de $X$ ser uma superfície parametrizada regular, de (4.3), usando a Proposição 1.3.8, segue que $\left(\operatorname{sen} \theta e_{1}^{0}-\cos \theta e_{3}^{0}\right)$ e $e_{2}^{0}$ são campos tangentes a ela e, portanto, podemos considerar o triedro móvel associado a $X$ dado por:

$$
\begin{aligned}
& e_{1}=\operatorname{sen} \theta e_{1}^{0}-\cos \theta e_{3}^{0}, \\
& e_{2}=e_{2}^{0}, \\
& e_{3}=\cos \theta e_{1}^{0}+\operatorname{sen} \theta e_{3}^{0} .
\end{aligned}
$$

De $d X=\omega_{1} e_{1}+\omega_{2} e_{2}$, resulta novamente de (4.3) que

$$
\begin{aligned}
& \omega_{1}=\operatorname{sen} \theta \omega_{1}^{0}-T d \theta-T \omega_{13}^{0}, \\
& \omega_{2}=\omega_{2}^{0}+T \cos \theta \omega_{12}^{0}-T \operatorname{sen} \theta \omega_{23}^{0},
\end{aligned}
$$

e, de (4.6), temos

$$
\begin{aligned}
& \omega_{12}=\left\langle d e_{1}, e_{2}\right\rangle=\operatorname{sen} \theta \omega_{12}^{0}+\cos \theta \omega_{23}^{0}, \\
& \omega_{13}=\left\langle d e_{1}, e_{3}\right\rangle=d \theta+\omega_{13}^{0}, \\
& \omega_{23}=\left\langle d e_{2}, e_{3}\right\rangle=-\cos \theta \omega_{12}^{0}+\operatorname{sen} \theta \omega_{23}^{0} .
\end{aligned}
$$




\subsection{Sobre as deformações das congruências de retas normais}

O próximo teorema nos garante que toda deformação de uma congruência de retas normal continua sendo uma congruência de retas normal.

Teorema 4.2.1 (Teorema de Beltrami). Seja

$$
X=X_{0}+T\left(\cos \theta e_{1}^{0}+\operatorname{sen} \theta e_{3}^{0}\right)
$$

uma congruência de retas normal a $X$. Considere uma superfície $\bar{X}_{0}=\tau\left(X_{0}\right)$, onde $\tau$ é uma isometria, e o triedro móvel a ela associada dado por:

$$
\bar{e}_{i}^{0}=d \tau\left(e_{i}^{0}\right), \quad i=1,2 ; \quad \bar{e}_{3}^{0}=\bar{e}_{1}^{0} \wedge \bar{e}_{2}^{0} .
$$

Então

$$
\bar{X}=\bar{X}_{0}+T\left(\cos \theta \bar{e}_{1}^{0}+\operatorname{sen} \theta \bar{e}_{3}^{0}\right)
$$

é uma congruência de retas normal a $\bar{X}$.

Demonstração. Como (4.9) é uma congruência de retas normal a $X$, do Lema 4.1.3, temos

$$
\cos \theta \omega_{1}^{0}+d T=0
$$

Como $\tau$ é uma isometria,

$$
\bar{\omega}_{1}^{0}=\left\langle d \bar{X}_{0}, \bar{e}_{1}^{0}\right\rangle=\left\langle d \tau \circ d X_{0}, d \tau\left(e_{1}^{0}\right)\right\rangle=\left\langle d X_{0}, e_{1}^{0}\right\rangle=\omega_{1}^{0} .
$$

Logo

$$
\cos \theta \bar{\omega}_{1}^{0}+d T=0
$$

de onde concluímos, novamente pelo Lema 4.1.3, que (4.10) é uma congruência de retas normal a $\bar{X}$.

Podemos resumir o Teorema de Beltrami com o diagrama:

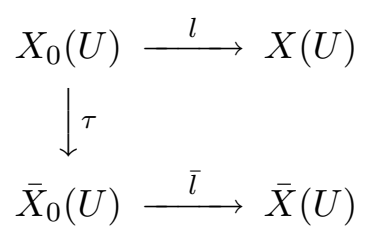

e denominamos $\bar{l}$ por congruência de retas associada a l e à isometria $\tau$. Dizemos também que $\bar{l}$ é uma deformação da congruência $l$.

Note que se $\left(\operatorname{sen} \theta \omega_{1}^{0}-T d \theta-T \omega_{13}^{0}\right)$ e $\left(\omega_{2}^{0}+T \cos \theta \omega_{12}^{0}-T \operatorname{sen} \theta \omega_{23}^{0}\right)$ forem 1-formas linearmente independentes, segue de (4.3) e da Proposição 1.3.8 que a aplicação $X$ é uma superfície parametrizada regular. Analogamente, $\bar{X}$ é uma superfície parametrizada regular se as respectivas 1-formas também forem linearmente independentes. Assim, salvo menções explícitas, vamos considerar este caso e denotaremos por $S=X(U), \bar{S}=\bar{X}(U), S_{0}=X_{0}(U)$ e $\bar{S}_{0}=\bar{X}_{0}(U)$.

Veremos, a seguir, uma primeira resposta ao Problema 4.0.5, no caso em que $S_{0}$ e $S$ são superfícies paralelas, sendo $S_{0}$ de curvatura gaussiana constante. 
Teorema 4.2.2. Seja $S_{0}$ uma superfície parametrizada regular de curvatura gaussiana constante $K_{0}$ e $l: S_{0} \rightarrow S$ uma congruência de retas normal a $S_{0}$ e $S$. Então,

(i) S é uma superfície de Weingarten, paralela a $S_{0}$, satisfazendo uma relação

$$
\psi(K, \mathrm{H})=0
$$

(ii) para toda isometria $\tau: S_{0} \rightarrow \bar{S}_{0}$, a congruência $\bar{l}$ (associada a $l$ e $\tau$ ) determina uma superfície de Weingarten $\bar{S}$ que satisfaz a mesma relação $\psi(\bar{K}, \overline{\mathrm{H}})=0$.

Demonstração. Sejam $X_{0}, X: U \subset \mathbb{R}^{2} \rightarrow \mathbb{R}^{3}$ parametrizações das superfícies $S_{0}$ e $S$, respectivamente, e denote por $\left\{e_{1}^{0}, e_{2}^{0}, e_{3}^{0}\right\}$ um triedro móvel associado a $X_{0}$.

Sendo $l$ é uma congruência de retas normal a $X_{0}$, temos

$$
X=X_{0}+T e_{3}^{0}
$$

Como $l$ é também normal a $X$, resulta

$$
0=\left\langle d X, e_{3}^{0}\right\rangle=\left\langle\left(\omega_{1}^{0}-T \omega_{13}^{0}\right) e_{1}^{0}+\left(\omega_{2}^{0}-T \omega_{23}^{0}\right) e_{2}^{0}+d T e_{3}^{0}, e_{3}^{0}\right\rangle=d T,
$$

isto é, $T$ é constante (não nula). Portanto, $X$ é uma superfície paralela a $X_{0}$ à distância $T$ e, da Proposição 3.1.7, suas curvaturas gaussiana e média são dadas por:

$$
K=\frac{K_{0}}{1-2 T \mathrm{H}_{0}+T^{2} K_{0}}, \quad \mathrm{H}=\frac{\mathrm{H}_{0}-T K_{0}}{1-2 T \mathrm{H}_{0}+T^{2} K_{0}} .
$$

Note que, isolando $\mathrm{H}_{0}$ da expressão de $\mathrm{H}$ e substituindo na de $K$, obtemos

$$
K-\frac{K_{0}(1+2 T \mathrm{H})}{1-T^{2} K_{0}}=0
$$

e, portanto, $X$ é uma superfície de Weingarten.

Considerando agora uma isometria $\tau: S_{0} \rightarrow \bar{S}_{0}$, a congruência de retas $\bar{l}$ é dada por:

$$
\bar{X}=\bar{X}_{0}+T \bar{e}_{3}^{0},
$$

sendo $\bar{e}_{3}^{0}$ um campo normal a $\bar{X}_{0}$. Assim, sendo a curvatura gaussiana de $\bar{X}_{0}, \bar{K}_{0}=K_{0}$, as curvaturas gaussiana e média de $\bar{X}$ satisfazem a relação

$$
\bar{K}-\frac{K_{0}(1+2 T \overline{\mathrm{H}})}{1-T^{2} K_{0}}=0
$$

o que termina a prova.

Um segundo resultado na direção do tratamento do Problema 4.0.5 é dado por:

Teorema 4.2.3 (Teorema de Weingarten). Sejam $S_{0}$ uma superfície de revolução e $l: S_{0} \rightarrow S$ uma congruência de retas tangente a $S_{0}$ na direção dos meridianos e normal a $S$. Então,

(i) S é uma superfície de revolução e de Weingarten, que satisfaz uma relação $\psi\left(\kappa_{1}, \kappa_{2}\right)=0$;

(ii) para toda isometria $\tau: S_{0} \rightarrow \bar{S}_{0}$, a congruência $\bar{l}$ (associada a l e $\tau$ ) é normal a uma superfície de Weingarten $\bar{S}$, que satisfaz a mesma relação $\psi\left(\bar{\kappa}_{1}, \bar{\kappa}_{2}\right)=0$. 
Demonstração. Sejam $X_{0}, X: U \subset \mathbb{R}^{2} \rightarrow \mathbb{R}^{3}$ parametrizações das superfícies $S_{0}$ e $S$, respectivamente, e considere $\left\{e_{1}^{0}, e_{2}^{0}, e_{3}^{0}\right\}$ um triedro móvel associado a $X_{0}$ tal que $e_{1}^{0}$ é um campo unitário tangente a $X_{0}$ na direção dos meridianos. A congruência $l$ é dada por

$$
X=X_{0}+T e_{1}^{0} .
$$

Não é restritivo supor que $X_{0}$ seja uma superfície de revolução ao redor do eixo $z$. Segue, do Exemplo 1.3 .4 e do fato que os coeficientes $E_{0}, F_{0}, G_{0}$ são funções somente do parâmetro $u$ da curva geradora de $X_{0}$, que

$$
\omega_{12}^{0}=B \omega_{2}^{0}
$$

onde $B(u)=\frac{\left(\sqrt{G_{0}}\right)_{u}}{\sqrt{E_{0} G_{0}}}$ é uma função diferenciável que só depende da curva geradora.

Como $l$ é também uma congruência de retas normal a $S$, do Lema 4.1.3, resulta que

$$
0=\omega_{1}^{0}+d T=\sqrt{E_{0}(u)} d u+d T .
$$

Portanto, $T=T(u)$, isto é, $T$ é constante ao longo dos paralelos. Disto segue que $S$ é também uma superfície de revolução.

Seja, como na Observação 4.1.4,

$$
e_{1}=-e_{3}^{0}, \quad e_{2}=e_{2}^{0}, \quad e_{3}=e_{1}^{0}
$$

o triedro móvel associado a $X$ e

$$
\begin{aligned}
\omega_{1} & =-T \omega_{13}^{0}, \\
\omega_{2} & =\omega_{2}^{0}+T \omega_{12}^{0}=(1+T B) \omega_{2}^{0}, \\
\omega_{12} & =\omega_{23}^{0}, \\
\omega_{13} & =\omega_{13}^{0}, \\
\omega_{23} & =-\omega_{12}^{0}=-B \omega_{2}^{0}
\end{aligned}
$$

as 1-formas associadas a este triedro. Destas relações segue que

$$
\omega_{13}=-\frac{1}{T} \omega_{1}, \quad \omega_{23}=-\frac{B}{1+T B} \omega_{2} .
$$

Logo, $e_{1}, e_{2}$ são campos de direções principais de $X$ e, da Proposição 1.3.6, as curvaturas principais de $X$ são dadas por:

$$
\kappa_{1}=-\frac{1}{T}, \quad \kappa_{2}=-\frac{B}{1+T B}
$$

e satisfazem a relação:

$$
\kappa_{1} \kappa_{2}+B\left(\kappa_{1}-\kappa_{2}\right)=0
$$

Considere, agora, a superfície $\bar{X}_{0}=\tau\left(X_{0}\right)$, isométrica a $X_{0}$, e

$$
\bar{e}_{i}^{0}=d \tau\left(e_{i}^{0}\right), \quad i=1,2 ; \quad \bar{e}_{3}^{0}=\bar{e}_{1}^{0} \wedge \bar{e}_{2}^{0}
$$

o triedro móvel associado a $\bar{X}_{0}$. A congruência de retas $\bar{l}$, associada a $l$ e $\tau$, é dada por:

$$
\bar{X}=\bar{X}_{0}+T \bar{e}_{1}^{0} .
$$

Como $\tau$ é uma isometria, $\bar{\omega}_{i}^{0}=\omega_{i}^{0}(i=1,2)$ e, portanto, da Proposição 1.2.2, $\bar{\omega}_{12}^{0}=\omega_{12}^{0}$. Logo,

$$
\bar{\omega}_{12}^{0}=B \bar{\omega}_{2}^{0}
$$

e, seguindo o mesmo raciocínio usado anteriormente, vemos que as curvaturas principais de $\bar{S}$ coincidem com $\kappa_{1}$ e $\kappa_{2}$ e, portanto, também satisfazem a relação (4.11). 


\subsubsection{Os Teoremas de Guichard}

A seguir, continuaremos o estudo do Problema 4.0.5 apresentando os teoremas de C. Guichard, estudados por L. Bianchi em [6] e que revelam a existência de novas soluções do problema para o caso em que $S_{0}$ é uma particular superfície de revolução e os segmentos da congruência de retas não saem mais, como no caso do Teorema de Weingarten, tangentes à superfície $S_{0}$.

Para tal, precisaremos destacar algumas superfícies de revolução.

Definição 4.2.4. (i) Parabolóide de revolução é obtido pela rotação da curva $x^{2}=2 a z$, onde $a>0$, em torno do eixo $z$. O ponto $\left(0,0, \frac{a}{2}\right)$, que é o foco de qualquer meridiano, é dito foco do parabolóide.

(ii) Elipsóide de revolução é obtido pela rotação da elipse

$$
\frac{x^{2}}{a^{2}}+\frac{z^{2}}{b^{2}}=1, \quad b>a>0,
$$

em torno do eixo $z$. Os pontos $(0,0, \pm c)$, onde $b^{2}=a^{2}+c^{2}$, que são os focos de qualquer meridiano, são denominados focos do elipsóide.

(iii) Hiperbolóide de revolução de duas folhas é obtido pela rotação da hipérbole

$$
\frac{z^{2}}{b^{2}}-\frac{x^{2}}{a^{2}}=1
$$

em torno do eixo $z$. Os pontos $(0,0, \pm c)$, onde $a^{2}+b^{2}=c^{2}$, que são os focos de qualquer meridiano, são ditos focos do hiperbolóide.

\section{O parabolóide de revolução é o I Teorema de Guichard}

Teorema 4.2.5 (I Teorema de Guichard (Parte A)). Seja $S_{0}$ um parabolóide de revolução e considere a aplicação

$$
\begin{aligned}
l: S_{0} & \longrightarrow \mathbb{R}^{3} \\
p & \longmapsto F,
\end{aligned}
$$

onde $F$ é foco de $S_{0}$. Então, para toda isometria $\tau: S_{0} \rightarrow \bar{S}_{0}$, a congruência de retas $\bar{l}$, associada a $l$ e $\tau$, é normal a uma superfície mínima.

Demonstração. A superfície $S_{0}$ é parametrizada por:

$$
X_{0}(x, v)=\left(x \cos v, x \operatorname{sen} v, \frac{x^{2}}{2 a}\right),
$$

com foco $F=\left(0,0, \frac{a}{2}\right), a>0$. Considere o seguinte triedro móvel associado a $X_{0}$ :

$$
\begin{aligned}
& e_{1}^{0}=-\frac{\left(X_{0}\right)_{x}}{\left\|\left(X_{0}\right)_{x}\right\|}=-\frac{1}{\sqrt{x^{2}+a^{2}}}(a \cos v, a \operatorname{sen} v, x), \\
& e_{2}^{0}=-\frac{\left(X_{0}\right)_{v}}{\left\|\left(X_{0}\right)_{v}\right\|}=(\operatorname{sen} v,-\cos v, 0), \\
& e_{3}^{0}=e_{1}^{0} \wedge e_{2}^{0}=\frac{1}{\sqrt{x^{2}+a^{2}}}(-x \cos v,-x \operatorname{sen} v, a),
\end{aligned}
$$


onde $e_{1}^{0}$ e $e_{2}^{0}$ são campos de direções principais pois são tangentes aos meridianos e paralelos de $X_{0}$, respectivamente. Então, da Proposição 1.3.3, as 1-formas associadas a este triedro são:

$$
\begin{aligned}
\omega_{1}^{0} & =-\frac{\sqrt{x^{2}+a^{2}}}{a} d x, \\
\omega_{2}^{0} & =-x d v, \\
\omega_{12}^{0} & =\frac{a}{\sqrt{x^{2}+a^{2}}} d v=B \omega_{2}^{0}, \quad B=-\frac{a}{x \sqrt{x^{2}+a^{2}}} .
\end{aligned}
$$

Como

$$
d \omega_{12}^{0}=-\frac{a x}{\left(x^{2}+a^{2}\right)^{\frac{3}{2}}} d x \wedge d v
$$

da equação de Gauss, $d \omega_{12}^{0}=-K_{0} \omega_{1}^{0} \wedge \omega_{2}^{0}$, segue que a curvatura gaussiana de $X_{0}$ é

$$
K_{0}=\frac{a^{2}}{\left(x^{2}+a^{2}\right)^{2}} .
$$

A aplicação $l$ é dada por

$$
F=X_{0}+T\left(\cos \theta e_{1}^{0}+\operatorname{sen} \theta e_{3}^{0}\right)
$$

onde $T$ é uma função diferenciável a valores reais não nula. Supondo $T>0$, como

$$
|T|=\left\|F-X_{0}\right\|=\frac{x^{2}+a^{2}}{2 a}
$$

resulta

$$
\begin{aligned}
T & =\frac{x^{2}+a^{2}}{2 a}, \\
\cos \theta & =\frac{\left\langle F-X_{0}, e_{1}^{0}\right\rangle}{T}=\frac{x}{\sqrt{x^{2}+a^{2}}}, \\
\operatorname{sen} \theta & =\frac{\left\langle F-X_{0}, e_{3}^{0}\right\rangle}{T}=\frac{a}{\sqrt{x^{2}+a^{2}}} .
\end{aligned}
$$

Mais ainda, observe que $l$ é uma congruência de retas normal a $F$ (pois $d F=0$ ).

Considere, agora, a superfície $\bar{X}_{0}=\tau\left(X_{0}\right)$, isométrica a $X_{0}$, e

$$
\bar{e}_{i}^{0}=d \tau\left(e_{i}^{0}\right), \quad i=1,2 ; \quad \bar{e}_{3}^{0}=\bar{e}_{1}^{0} \wedge \bar{e}_{2}^{0}
$$

o triedro móvel associado a esta superfície. A congruência de retas $\bar{l}$, associada a $l$ e $\tau$, dada por

$$
\bar{X}=\bar{X}_{0}+T\left(\cos \theta \bar{e}_{1}^{0}+\operatorname{sen} \theta \bar{e}_{3}^{0}\right)
$$

é normal a $\bar{X}$, pelo Teorema de Beltrami. Vamos mostrar que $\bar{X}$ é uma superfície mínima. Da Observação 4.1.4, considerando o triedro móvel associado a $\bar{X}$ dado por:

$$
\begin{aligned}
& \bar{e}_{1}=\operatorname{sen} \theta \bar{e}_{1}^{0}-\cos \theta \bar{e}_{3}^{0}, \\
& \bar{e}_{2}=\bar{e}_{2}^{0}, \\
& \bar{e}_{3}=\cos \theta \bar{e}_{1}^{0}+\operatorname{sen} \theta \bar{e}_{3}^{0},
\end{aligned}
$$


as 1-formas a ele associadas são:

$$
\begin{aligned}
\bar{\omega}_{1} & =\operatorname{sen} \theta \bar{\omega}_{1}^{0}-T d \theta-T \bar{\omega}_{13}^{0}, \\
\bar{\omega}_{2} & =\bar{\omega}_{2}^{0}+T \cos \theta \bar{\omega}_{12}^{0}-T \operatorname{sen} \theta \bar{\omega}_{23}^{0}, \\
\bar{\omega}_{13} & =d \theta+\bar{\omega}_{13}^{0}, \\
\bar{\omega}_{23} & =-\cos \theta \bar{\omega}_{12}^{0}+\operatorname{sen} \theta \bar{\omega}_{23}^{0} .
\end{aligned}
$$

Note que, utilizando (4.14), (4.16) e (4.15), podemos verificar as seguintes relações:

$$
\begin{aligned}
& 1+2 T B \cos \theta=0, \\
& 2 T d \theta-\operatorname{sen} \theta \omega_{1}^{0}=0, \\
& B \cos \theta+2 T K_{0}=0 .
\end{aligned}
$$

Além disso, como $\tau$ é uma isometria, $\bar{\omega}_{i}^{0}=\omega_{i}^{0}, i=1,2$, e $\bar{\omega}_{12}^{0}=\omega_{12}^{0}$. Assim, utilizando (4.18) e (4.19), obtemos

$$
\begin{aligned}
\bar{\omega}_{1} \wedge \bar{\omega}_{23}+\bar{\omega}_{13} \wedge \bar{\omega}_{2}= & \left(\operatorname{sen} \theta \omega_{1}^{0}-T d \theta-T \bar{\omega}_{13}^{0}\right) \wedge\left(-\cos \theta \omega_{12}^{0}+\operatorname{sen} \theta \bar{\omega}_{23}^{0}\right) \\
& +\left(d \theta+\bar{\omega}_{13}^{0}\right) \wedge\left(\omega_{2}^{0}+T \cos \theta \omega_{12}^{0}-T \operatorname{sen} \theta \bar{\omega}_{23}^{0}\right) \\
= & \left(2 T d \theta-\operatorname{sen} \theta \omega_{1}^{0}\right) \wedge\left(\cos \theta \omega_{2}^{0}-\operatorname{sen} \theta \bar{\omega}_{23}^{0}\right) \\
& +(1+2 T B \cos \theta) \bar{\omega}_{13}^{0} \wedge \omega_{2}^{0}+d \theta \wedge \omega_{2}^{0}-2 T \operatorname{sen} \theta \bar{\omega}_{13}^{0} \wedge \bar{\omega}_{23}^{0} \\
= & \left(d \theta-2 T \operatorname{sen} \theta K_{0} \omega_{1}^{0}\right) \wedge \omega_{2}^{0} \\
= & -\operatorname{sen} \theta\left(B \cos \theta+2 T K_{0}\right) \omega_{1}^{0} \wedge \omega_{2}^{0}=0,
\end{aligned}
$$

onde, na terceira igualdade, foi usada a equação de Gauss:

$$
\bar{\omega}_{13}^{0} \wedge \bar{\omega}_{23}^{0}=\bar{K}_{0} \omega_{1}^{0} \wedge \omega_{2}^{0}=K_{0} \omega_{1}^{0} \wedge \omega_{2}^{0} .
$$

Portanto, da Proposição 1.3.2, concluímos que $\bar{X}$ é mínima.

Teorema 4.2.6 (I Teorema de Guichard (Parte B)). Sejam $S_{0}$ um parabolóide de revolução e $l^{s}$ a aplicação:

$$
\begin{aligned}
l^{s}: S_{0} & \longrightarrow \mathbb{R}^{3} \\
p & \longmapsto F_{p}^{s},
\end{aligned}
$$

onde $F_{p}^{s}$ denota o ponto simétrico ao foco $F$ em relação ao plano tangente em $p$ a $S_{0}$. Então,

(i) a congruência de retas $l^{s}$ é normal a uma superfície mínima;

(ii) para toda isometria $\tau: S_{0} \rightarrow \bar{S}_{0}$, a congruência de retas $\bar{l}^{s}$ (associada a $l^{s}$ e $\tau$ ) é normal a uma superfície mínima.

Demonstração. Considere o triedro móvel associado a $X_{0}$ dado por (4.13) e as 1-formas (4.14). A aplicação $l^{s}$ é dada por:

$$
X^{s}=X_{0}+T\left(\cos \theta e_{1}^{0}-\operatorname{sen} \theta e_{3}^{0}\right),
$$

onde $T$ e $\theta$ são definidas por (4.16). Como $\cos \theta \omega_{1}^{0}+d T=0$ segue, do Lema 4.1.3, que $l^{s}$ é uma congruência de retas normal a $X^{s}$. Além disso, usando (4.13), (4.16) e (4.12), temos

$$
X^{s}=X_{0}+T\left(\cos \theta e_{1}^{0}-\operatorname{sen} \theta e_{3}^{0}\right)=\left(x \cos v, x \operatorname{sen} v,-\frac{a}{2}\right),
$$




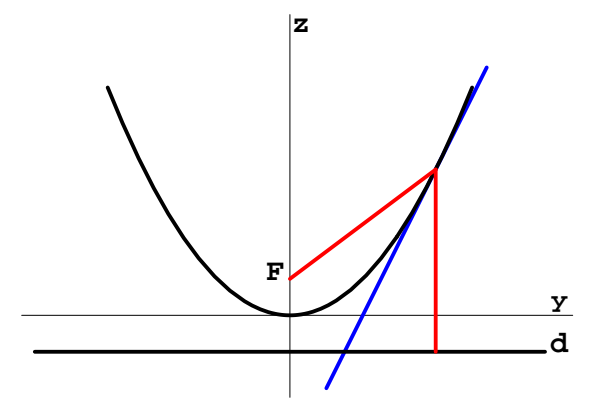

ou seja, $X^{s}$ é o plano $z=-\frac{a}{2}$ e, portanto, é uma superfície mínima.

Considere, agora, uma superfície $\bar{X}_{0}=\tau\left(X_{0}\right)$, isométrica a $X_{0}$, e o triedro móvel a ela associado dado por:

$$
\bar{e}_{i}^{0}=d \tau\left(e_{i}^{0}\right), \quad i=1,2 ; \quad \bar{e}_{3}^{0}=\bar{e}_{1}^{0} \wedge \bar{e}_{2}^{0} .
$$

Do Teorema de Beltrami, temos que a congruência de retas $\bar{l}^{s}$ (associada a $l^{s}$ e $\tau$ ) que é dada por:

$$
\bar{X}^{s}=\bar{X}_{0}+T\left(\cos \theta \bar{e}_{1}^{0}-\operatorname{sen} \theta \bar{e}_{3}^{0}\right),
$$

é uma congruência de retas normal a $\bar{X}^{s}$. Observe que esta congruência equivale a dada por (4.17), substituindo $\theta$ por $(-\theta)$. Assim, como continuam valendo as relações (4.19), resulta

$$
\bar{\omega}_{1}^{s} \wedge \bar{\omega}_{23}^{s}+\bar{\omega}_{13}^{s} \wedge \bar{\omega}_{2}^{s}=0
$$

isto é, $\bar{X}^{s}$ é uma superfície mínima.

Se $X_{0}: U \subset \mathbb{R}^{2} \rightarrow \mathbb{R}^{3}$ for um parabolóide de revolução, resulta dos Teoremas 4.2.5 e 4.2.6 que, dada uma isometria $\tau: X_{0}(U) \rightarrow \bar{X}_{0}(U)$, as aplicações definidas por

$$
\bar{X}=\bar{X}_{0}+T\left(\cos \theta \bar{e}_{1}^{0}+\operatorname{sen} \theta \bar{e}_{3}^{0}\right) \quad \text { e } \quad \bar{X}^{s}=\bar{X}_{0}+T\left(\cos \theta \bar{e}_{1}^{0}-\operatorname{sen} \theta \bar{e}_{3}^{0}\right)
$$

são superfícies mínimas, sendo $\theta$ e $T$ dadas por (4.16), e $\left\{\bar{e}_{1}^{0}, \bar{e}_{2}^{0}, \bar{e}_{3}^{0}\right\}$ um triedro móvel associado a $\bar{X}_{0}, \bar{e}_{1}^{0}, \bar{e}_{2}^{0}$ os campos tangentes aos meridianos e aos paralelos de $\bar{X}_{0}$, respectivamente. Além disso, para cada $q \in U$, os pontos $\bar{X}(q)$ e $\bar{X}^{s}(q)$ são simétricos com relação ao plano tangente da superfície $\bar{X}_{0}$ em $q$. Sendo assim, $\bar{X}$ e $\bar{X}^{s}$ são ditas superfícies simétricas em relação a $\bar{X}_{0}$. Logo, foi provado o seguinte

Corolário 4.2.7. Para toda superfície $\bar{S}_{0}$, isométrica a um parabolóide de revolução, podemos associar duas superfícies mínimas, simétricas em relação a $\bar{S}_{0}$.

\section{O elipsóide de revolução e o hiperbolóide de revolução de duas folhas; o II Teorema de Guichard}

Teorema 4.2.8 (II Teorema de Guichard (Parte A)). Seja $S_{0}$ um elipsóide de revolução ou um hiperbolóide de revolução de duas folhas, cujo eixo maior tem comprimento $2 b$, e considere a aplicação

$$
\begin{aligned}
l: S_{0} & \longrightarrow \mathbb{R}^{3} \\
p & \longmapsto F,
\end{aligned}
$$


onde $F$ é um dos focos de $S_{0}$. Então, para toda isometria $\tau: S_{0} \rightarrow \bar{S}_{0}$, a congruência de retas $\bar{l}$ (associada a l e $\tau$ ) é normal a uma superfície de curvatura média constante de módulo igual a $\frac{1}{2 b}$.

Demonstração. Vamos provar este teorema no caso em que $S_{0}$ é um elipsóide de revolução, pois o caso de $S_{0}$ ser um hiperbolóide de revolução de duas folhas segue análogo.

Considere a parametrização de $S_{0}$ dada por:

$$
X_{0}(z, v)=(x \cos v, x \operatorname{sen} v, z)
$$

onde $x=x(z)>0$ é tal que

$$
\frac{x^{2}}{a^{2}}+\frac{z^{2}}{b^{2}}=1, \quad b>a>0
$$

e $F=(0,0, \pm c)$ são os focos de $X_{0}\left(b^{2}=a^{2}+c^{2}\right)$. Considere, associado a $X_{0}$, o triedro móvel:

$$
\begin{aligned}
& e_{1}^{0}=\frac{\left(X_{0}\right)_{z}}{\left\|\left(X_{0}\right)_{z}\right\|}=\frac{1}{\sqrt{1+\left(x^{\prime}\right)^{2}}}\left(x^{\prime} \cos v, x^{\prime} \operatorname{sen} v, 1\right), \\
& e_{2}^{0}=-\frac{\left(X_{0}\right)_{v}}{\left\|\left(X_{0}\right)_{v}\right\|}=(\operatorname{sen} v,-\cos v, 0), \\
& e_{3}^{0}=e_{1}^{0} \wedge e_{2}^{0}=\frac{1}{\sqrt{1+\left(x^{\prime}\right)^{2}}}\left(\cos v, \operatorname{sen} v,-x^{\prime}\right),
\end{aligned}
$$

onde $x^{\prime}$ denota a derivada de $x$ em relação a $z$ e $e_{1}^{0}, e_{2}^{0}$ são campos de direções principais. Segue da Proposição 1.3.3 que as 1-formas associadas a este triedro são

$$
\begin{aligned}
\omega_{1}^{0} & =\sqrt{1+\left(x^{\prime}\right)^{2}} d z, \\
\omega_{2}^{0} & =-x d v \\
\omega_{12}^{0} & =\frac{-x^{\prime}}{\sqrt{1+\left(x^{\prime}\right)^{2}}} d v=B \omega_{2}^{0}, \quad B=\frac{x^{\prime}}{x \sqrt{1+\left(x^{\prime}\right)^{2}}} .
\end{aligned}
$$

Como, pela equação de Gauss,

$$
-K_{0} \omega_{1}^{0} \wedge \omega_{2}^{0}=d \omega_{12}^{0}=-\frac{x^{\prime \prime}}{\left(1+\left(x^{\prime}\right)^{2}\right)^{\frac{3}{2}}} d z \wedge d v,
$$

então a curvatura gaussiana de $X_{0}$ é:

$$
K_{0}=\frac{-x^{\prime \prime}}{x\left(\left(1+\left(x^{\prime}\right)^{2}\right)^{2}\right.}=\frac{a^{4} b^{2}}{\left(a^{4}+c^{2} x^{2}\right)^{2}},
$$

onde foi usada a (4.21).

A aplicação $l$ é dada por:

$$
F=X_{0}+T\left(\cos \theta e_{1}^{0}+\operatorname{sen} \theta e_{3}^{0}\right),
$$

onde $F$ é um dos focos do elipsóide de revolução $X_{0}$. Mais ainda, como $d F=0, l$ é trivialmente uma congruência de retas normal a $F$. Sendo $F=(0,0, \pm c)$, temos

$$
|T|=\left\|F-X_{0}\right\|=\frac{b^{2} \mp c z}{b},
$$


de onde, considerando $T>0$, obtemos

$$
\begin{aligned}
T & =\frac{b^{2} \mp c z}{b}, \\
\cos \theta & =\frac{\left\langle F-X_{0}, e_{1}^{0}\right\rangle}{T}=\frac{ \pm c x}{\sqrt{a^{4}+c^{2} x^{2}}}, \\
\operatorname{sen} \theta & =\frac{\left\langle F-X_{0}, e_{3}^{0}\right\rangle}{T}=\frac{-a^{2}}{\sqrt{a^{4}+c^{2} x^{2}}} .
\end{aligned}
$$

Considere, agora, uma superfície $\bar{X}_{0}=\tau\left(X_{0}\right)$, isométrica a $X_{0}$, e o seguinte triedro móvel a ela associado:

$$
\bar{e}_{i}^{0}=d \tau\left(e_{i}^{0}\right), \quad i=1,2 ; \quad \bar{e}_{3}^{0}=\bar{e}_{1}^{0} \wedge \bar{e}_{2}^{0} .
$$

A congruência de retas $\bar{l}$ (associada a $l$ e $\tau$ ) dada por:

$$
\bar{X}=\bar{X}_{0}+T\left(\cos \theta \bar{e}_{1}^{0}+\operatorname{sen} \theta \bar{e}_{3}^{0}\right),
$$

é normal a $\bar{X}$, pelo Teorema de Beltrami. Vamos mostrar que $\bar{X}$ é uma superfície de curvatura média constante igual a $\left(-\frac{1}{2 b}\right)$. Considere, como na Observação 4.1.4, $\left\{\bar{e}_{1}, \bar{e}_{2}, \bar{e}_{3}\right\}$ o triedro móvel associado a $\bar{X}$ e $\bar{\omega}_{1}, \bar{\omega}_{2}, \bar{\omega}_{i j}, i, j=1,2,3$, as 1 -formas a ele associadas. Observe que, usando (4.23), (4.24) e (4.26), temos as seguintes relações:

$$
\begin{aligned}
& {\left[(T-b)^{2}-b^{2}\right] d \theta-(T-b) \operatorname{sen} \theta \omega_{1}^{0}=0,} \\
& {\left[(T-b)^{2}-b^{2}\right] B \cos \theta+T-b=0,} \\
& {\left[(T-b)^{2}-b^{2}\right] K_{0}+(T-b) B \cos \theta+1=0 .}
\end{aligned}
$$

Mais ainda, $\bar{\omega}_{i}^{0}=\omega_{i}^{0}, i=1,2, \bar{\omega}_{12}^{0}=\omega_{12}^{0}$, pois $\tau$ é uma isometria. Logo, de (4.28), resulta

$$
\begin{aligned}
\bar{\omega}_{1} \wedge \bar{\omega}_{23}+ & \bar{\omega}_{13} \wedge \bar{\omega}_{2}+\frac{1}{b} \bar{\omega}_{1} \wedge \bar{\omega}_{2}= \\
= & \frac{1}{b} \operatorname{sen} \theta\left[(T-b) B \cos \theta+T(T-2 b) K_{0}+1\right] \omega_{1}^{0} \wedge \omega_{2}^{0} \\
& +\frac{1}{b} \operatorname{sen} \theta\left[(b-T) \operatorname{sen} \theta \omega_{1}^{0}+T(T-2 b) d \theta\right] \wedge \bar{w}_{23}^{0} \\
& +\frac{1}{b}[(2 b-T) T B \cos \theta+b-T]\left(d \theta \wedge \omega_{2}^{0}+\bar{\omega}_{13}^{0} \wedge \omega_{2}^{0}\right)=0,
\end{aligned}
$$

onde usamos que $\omega_{12}^{0}=B \omega_{2}^{0}$ e a equação de Gauss:

$$
\bar{\omega}_{13}^{0} \wedge \bar{\omega}_{23}^{0}=\bar{K}_{0} \bar{\omega}_{1}^{0} \wedge \bar{\omega}_{2}^{0}=K_{0} \omega_{1}^{0} \wedge \omega_{2}^{0} .
$$

Portanto, como

$$
\bar{\omega}_{1} \wedge \bar{\omega}_{23}+\bar{\omega}_{13} \wedge \bar{\omega}_{2}=-\frac{1}{b} \bar{\omega}_{1} \wedge \bar{\omega}_{2}
$$

pela Proposição 1.3.2, $\bar{X}$ tem curvatura média igual a $\left(-\frac{1}{2 b}\right)$.

Teorema 4.2.9 (II Teorema de Guichard (Parte B)). Sejam $S_{0}$ um elipsóide de revolução ou um hiperbolóide de revolução de duas folhas, com eixo maior de comprimento $2 b$, e $l^{s}$ a aplicação

$$
\begin{aligned}
l^{s}: S_{0} & \longrightarrow \mathbb{R}^{3} \\
p & \longmapsto F_{p}^{s},
\end{aligned}
$$

onde $F_{p}^{s}$ é o ponto simétrico a um dos focos de $S_{0}$ em relação ao seu plano tangente em $p$. Então, 
(i) a congruência de retas $l^{s}$ é normal a uma superfície de curvatura média constante de módulo igual a $\frac{1}{2 b}$;

(ii) para toda isometria $\tau: S_{0} \rightarrow \bar{S}_{0}$, a congruência de retas $\bar{l}^{s}$ (associada a $l^{s}$ e $\tau$ ) é normal a uma superfície de mesma curvatura média (em módulo).

Demonstração. Novamente faremos a prova considerando $S_{0}$ um elipsóide de revolução. Para $S_{0}$ sendo um hiperbolóide de revolução de duas folhas a demonstração segue análoga.

Considerando a superfície $S_{0}$ parametrizada por (4.20), as equações (4.22), (4.23), (4.26) e o foco $F=(0,0, \pm c)$, a congruência $l^{s}$ que é dada por:

$$
X^{s}=X_{0}+T\left(\cos \theta e_{1}^{0}-\operatorname{sen} \theta e_{3}^{0}\right)
$$

equivale a (4.25), trocando $\theta$ por $(-\theta)$. Então, como

$$
\cos \theta \omega_{1}^{0}+d T=0
$$

pelo Lema 4.1.3, $l^{s}$ é uma congruência de retas normal a $X^{s}$.

Note que, usando (4.20), (4.22), (4.26) e (4.29) temos

$$
\begin{aligned}
X^{s}(z, v) & =X_{0}+T\left(\cos \theta e_{1}^{0}-\operatorname{sen} \theta e_{3}^{0}\right) \\
& =\left(\frac{2 a b \sqrt{b^{2}-z^{2}}}{b^{2} \pm c z} \cos u, \frac{2 a b \sqrt{b^{2}-z^{2}}}{b^{2} \pm c z} \operatorname{sen} u, \frac{z\left(a^{2}+b^{2}\right) \pm c b^{2}}{b^{2} \pm c z}\right),
\end{aligned}
$$

Denotando $X^{s}=(\bar{x}, \bar{y}, \bar{z})$, de

$$
\bar{x}^{2}+\bar{y}^{2}+(\bar{z} \pm c)^{2}=4 b^{2},
$$

segue que $X^{s}$ é a esfera de centro $(0,0, \mp c)$ e raio $2 b$, que tem curvatura média constante de módulo igual a $\frac{1}{2 b}$.

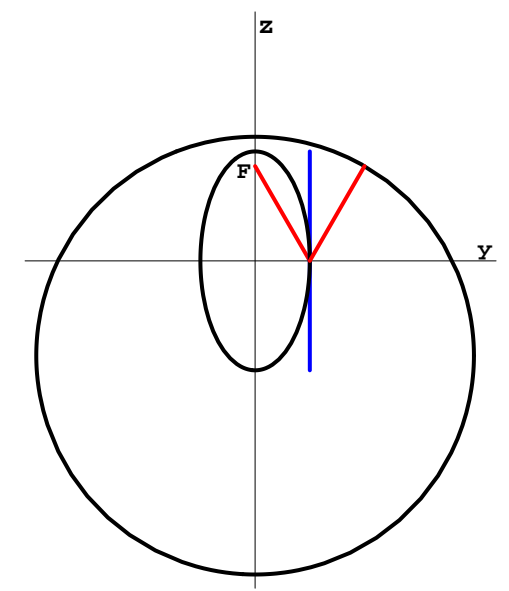

Considerando uma superfície $\bar{X}_{0}=\tau\left(X_{0}\right)$, isométrica a $X_{0}$, e o triedro móvel a ela associado dado por:

$$
\bar{e}_{i}^{0}=d \tau\left(e_{i}^{0}\right), \quad i=1,2 ; \quad \bar{e}_{3}^{0}=\bar{e}_{1}^{0} \wedge \bar{e}_{2}^{0},
$$

a congruência $\bar{l}^{s}$ (associada a $l^{s}$ e $\tau$ ) é dada por:

$$
\bar{X}^{s}=\bar{X}_{0}+T\left(\cos \theta \bar{e}_{1}^{0}-\operatorname{sen} \theta \bar{e}_{3}^{0}\right)
$$


e é normal a $\bar{X}^{s}$, pelo Teorema de Beltrami. Como as relações (4.28) valem também substituindo $(-\theta)$ em $\theta$, segue da demonstração do Teorema 4.2 .8 que $\bar{X}^{s}$ possui curvatura média constante e igual a $-\frac{1}{2 b}$.

Observação 4.2.10. A congruência de retas

$$
X=X_{0}+T \xi
$$

é normal à superfície $X$ de curvatura média constante $\mathrm{H} \neq 0$ se, e somente se,

$$
\tilde{X}=X+\frac{1}{2 \mathrm{H}} \xi=X_{0}+\left(T+\frac{1}{2 \mathrm{H}}\right) \xi
$$

define uma congruência de retas normal à superfície $\tilde{X}$ de curvatura gaussiana constante $4 \mathrm{H}^{2}$. Isto decorre do Teorema de Beltrami e de:

$$
\langle d \tilde{X}, \xi\rangle=\langle d X, \xi\rangle
$$

Assim, como as superfícies

$$
\begin{aligned}
\bar{X} & =\bar{X}_{0}+T\left(\cos \theta \bar{e}_{1}^{0}+\operatorname{sen} \theta \bar{e}_{3}^{0}\right), \\
\bar{X}^{s} & =\bar{X}_{0}+T\left(\cos \theta \bar{e}_{1}^{0}-\operatorname{sen} \theta \bar{e}_{3}^{0}\right),
\end{aligned}
$$

obtidas nos Teoremas 4.2.8 e 4.2.9, possuem curvatura média constante e igual a $\left(-\frac{1}{2 b}\right)$, temos que as superfícies dadas por

$$
\begin{aligned}
\tilde{X} & =\bar{X}_{0}+(T-b)\left(\cos \theta \bar{e}_{1}^{0}+\operatorname{sen} \theta \bar{e}_{3}^{0}\right), \\
\tilde{X}^{s} & =\bar{X}_{0}+(T-b)\left(\cos \theta \bar{e}_{1}^{0}-\operatorname{sen} \theta \bar{e}_{3}^{0}\right),
\end{aligned}
$$

são de curvatura gaussiana constante igual a $\frac{1}{b^{2}}$.

Resulta desta observação o seguinte:

Corolário 4.2.11. A cada superfície $\bar{S}_{0}$, isométrica a um elipsóide de revolução ou a um hiperbolóide de revolução de duas folhas, podemos associar duas superfície de curvatura gaussiana constante e dois pares de superfícies de curvatura média constante não nula (um para cada foco), que são simétricas em relação a $\bar{S}_{0}$.

Definição 4.2.12. Denominaremos as congruências de retas,

$$
\bar{X}=\bar{X}_{0}+T\left(\cos \theta \bar{e}_{1}^{0}+\operatorname{sen} \theta \bar{e}_{3}^{0}\right), \quad \bar{X}^{s}=\bar{X}_{0}+T\left(\cos \theta \bar{e}_{1}^{0}-\operatorname{sen} \theta \bar{e}_{3}^{0}\right),
$$

obtidas nos Teoremas de Guichard, sendo $\bar{X}_{0}$ é isométrica a uma das superfícies de revolução da Definição 4.2.4, por congruências de Guichard, onde as funções $T$ e $\theta$ satisfazem às seguintes relações: 


\begin{tabular}{|c||c|c|}
\hline & $T$ & $\tan \theta$ \\
\hline \hline Parabolóide de revolução & $\frac{x^{2}+a^{2}}{2 a}$ & $\frac{a}{x}$ \\
\hline $\begin{array}{c}\text { Elipsóide de revolução } \\
\text { ou hiperbolóide de } \\
\text { revolução de duas folhas }\end{array}$ & $\frac{b^{2} \mp c z}{b}$ & $\mp \frac{a^{2}}{c x}$ \\
\hline
\end{tabular}

\subsection{Recíproca dos Teoremas de Guichard}

Na presente seção trataremos do Problema 4.0.5 para o caso em que a superfície de Weingarten $S$ seja de curvatura média $\mathrm{H}$ constante ou de curvatura gaussiana $K$ constante não nula. Em outras palavras, consideraremos uma congruência de retas $l: S_{0} \rightarrow S$ normal a uma superfície $S$, impondo que qualquer sua deformação $\bar{l}: \bar{S}_{0} \rightarrow \bar{S}$ (com $\tau: S_{0} \rightarrow \bar{S}_{0}$ isometria) seja normal a uma superfície de curvatura média $\overline{\mathrm{H}}=\mathrm{H}$ ou de curvatura gaussiana $\bar{K}=K$.

Tais resultados foram obtidos por L. Bianchi no trabalho [6], provando que excluindo o caso $K<0$ as únicas soluções deste problema (quando $l$ não é tangente à superfície $S_{0}$ ) são dadas pelos Teoremas de Guichard, ou seja, a congruência $l$ é uma congruência de Guichard.

\subsubsection{Tratamento do Problema 4.0.5 para o caso $\mathrm{H}=0$}

Teorema 4.3.1. Seja l $: S_{0} \rightarrow S$ uma congruência de retas normal a uma superfície mínima $S$ tal que, para toda isometria $\tau: S_{0} \rightarrow \bar{S}_{0}$, a congruência $\bar{l}$ (associada a $l$ e $\tau$ ) seja normal a uma superfície mínima $\bar{S}$. Se as retas determinadas pela congruência l não forem tangentes à superfície $S_{0}$, então

(i) $S_{0}$ é isométrica a um parabolóide de revolução;

(ii) l é uma congruência de Guichard.

Demonstração. Primeiro, vamos obter algumas relações e, em seguida, usaremos as mesmas para provar a tese.

Indicando com $\left\{e_{1}^{0}, e_{2}^{0}, e_{3}^{0}\right\}$ um triedro móvel associado a $S_{0}$ e $\bar{e}_{i}^{0}=d \tau\left(e_{i}^{0}\right), i=1,2, \bar{e}_{3}^{0}=\bar{e}_{1}^{0} \wedge \bar{e}_{2}^{0}$, as congruências $l$ e $\bar{l}$ são dadas por:

$$
\begin{aligned}
& X=X_{0}+T\left(\cos \theta e_{1}^{0}+\operatorname{sen} \theta e_{3}^{0}\right), \\
& \bar{X}=\bar{X}_{0}+T\left(\cos \theta \bar{e}_{1}^{0}+\operatorname{sen} \theta \bar{e}_{3}^{0}\right) .
\end{aligned}
$$

Considerando $\omega_{1}^{0}, \omega_{2}^{0}, \omega_{i j}^{0}$ e $\bar{\omega}_{1}^{0}, \bar{\omega}_{2}^{0}, \bar{\omega}_{i j}^{0}, i, j=1,2,3$, as 1-formas associadas aos triedros de $X_{0}$ e $\bar{X}_{0}$, respectivamente, temos que $\omega_{i}^{0}=\bar{\omega}_{i}^{0}, i=1,2$, e $\omega_{12}^{0}=\bar{\omega}_{12}^{0}$. Detonando por:

$$
\begin{aligned}
d \theta & =\theta_{1}^{0} \omega_{1}^{0}+\theta_{2}^{0} \omega_{2}^{0}, \\
\omega_{12}^{0} & =A \omega_{1}^{0}+B \omega_{2}^{0}, \\
\bar{\omega}_{13}^{0} & =\bar{h}_{11}^{0} \omega_{1}^{0}+\bar{h}_{12}^{0} \omega_{2}^{0}, \\
\bar{\omega}_{23}^{0} & =\bar{h}_{21}^{0} \omega_{1}^{0}+\bar{h}_{22}^{0} \omega_{2}^{0},
\end{aligned}
$$


onde $\theta_{i}^{0}, A, B, \bar{h}_{i j}^{0}, i, j=1,2$, são funções em $U$ a valores reais e $\bar{h}_{21}^{0}=\bar{h}_{12}^{0}$.

Considere o triedro móvel associado a $\bar{X}$ dado na Observação 4.1.4:

$$
\begin{aligned}
& \bar{e}_{1}=\operatorname{sen} \theta \bar{e}_{1}^{0}-\cos \theta \bar{e}_{3}^{0}, \\
& \bar{e}_{2}=\bar{e}_{2}^{0} \\
& \bar{e}_{3}=\cos \theta \bar{e}_{1}^{0}+\operatorname{sen} \theta \bar{e}_{3}^{0}
\end{aligned}
$$

e

$$
\begin{aligned}
\bar{\omega}_{1} & =\operatorname{sen} \theta \bar{\omega}_{1}^{0}-T d \theta-T \bar{\omega}_{13}^{0}, \\
\bar{\omega}_{2} & =\bar{\omega}_{2}^{0}+T \cos \theta \bar{\omega}_{12}^{0}-T \operatorname{sen} \theta \bar{\omega}_{23}^{0}, \\
\bar{\omega}_{12} & =\operatorname{sen} \theta \bar{\omega}_{12}^{0}+\cos \theta \bar{\omega}_{23}^{0}, \\
\bar{\omega}_{13} & =d \theta+\bar{\omega}_{13}^{0}, \\
\bar{\omega}_{23} & =-\cos \theta \bar{\omega}_{12}^{0}+\operatorname{sen} \theta \bar{\omega}_{23}^{0}
\end{aligned}
$$

as 1-formas associadas a este triedro.

Como, para toda superfície isométrica a $X_{0}, \bar{X}$ é mínima, usando (4.30) e (4.31), segue da Proposição 1.3.2 que

$$
\begin{aligned}
0= & \bar{\omega}_{1} \wedge \bar{\omega}_{23}+\bar{\omega}_{13} \wedge \bar{\omega}_{2} \\
= & \left(\operatorname{sen} \theta \omega_{1}^{0}-T d \theta-T \bar{\omega}_{13}^{0}\right) \wedge\left(-\cos \theta \omega_{12}^{0}+\operatorname{sen} \theta \bar{\omega}_{23}^{0}\right) \\
& +\left(d \theta+\bar{\omega}_{13}^{0}\right) \wedge\left(\omega_{2}^{0}+T \cos \theta \omega_{12}^{0}-T \operatorname{sen} \theta \bar{\omega}_{23}^{0}\right) \\
= & {\left[(2 B T \cos \theta+1) \bar{h}_{11}^{0}+2 T\left(\theta_{2}^{0} \operatorname{sen} \theta-A \cos \theta\right) \bar{h}_{12}^{0}+\operatorname{sen} \theta\left(\operatorname{sen} \theta-2 T \theta_{1}^{0}\right) \bar{h}_{22}^{0}\right.} \\
+ & \left.\left(-B \operatorname{sen} \theta \cos \theta+2 B T \theta_{1}^{0} \cos \theta-2 A T \theta_{2}^{0} \cos \theta-2 T K_{0} \operatorname{sen} \theta+\theta_{1}^{0}\right)\right] \omega_{1}^{0} \wedge \omega_{2}^{0},
\end{aligned}
$$

onde, na terceira igualdade usamos $\bar{\omega}_{13}^{0} \wedge \bar{\omega}_{23}^{0}=\bar{K}_{0} \bar{\omega}_{1}^{0} \wedge \bar{\omega}_{2}^{0}=K_{0} \omega_{1}^{0} \wedge \omega_{2}^{0}$. Portanto, para todo $\bar{h}_{i j}^{0}, i, j=1,2$, vale a seguinte relação

$$
\begin{aligned}
& (2 B T \cos \theta+1) \bar{h}_{11}^{0}+2 T\left(\theta_{2}^{0} \operatorname{sen} \theta-A \cos \theta\right) \bar{h}_{12}^{0}+\operatorname{sen} \theta\left(\operatorname{sen} \theta-2 T \theta_{1}^{0}\right) \bar{h}_{22}^{0} \\
& +\left(-B \operatorname{sen} \theta \cos \theta+2 B T \theta_{1}^{0} \cos \theta-2 A T \theta_{2}^{0} \cos \theta-2 T K_{0} \operatorname{sen} \theta+\theta_{1}^{0}\right)=0,
\end{aligned}
$$

de onde, anulando os coeficientes de $\bar{h}_{i j}^{0}$ e o termo independente, obtemos:

$$
\begin{aligned}
& 2 T B \cos \theta+1=0, \\
& \theta_{2}^{0} \operatorname{sen} \theta-A \cos \theta=0, \\
& \operatorname{sen} \theta-2 \theta_{1}^{0} T=0, \\
& \operatorname{sen} \theta\left(B \cos \theta+2 T K_{0}\right)+2 A T \theta_{2}^{0} \cos \theta=0 .
\end{aligned}
$$

Note que, foi usado o fato de a congruência de retas $l$ não ser tangente a $X_{0}$ implicar em $\operatorname{sen} \theta \neq 0$. Além disso, da primeira equação, temos também que $\cos \theta \neq 0$. Assim, usando a equação de estrutura $d \omega_{1}^{0}=\omega_{2}^{0} \wedge \omega_{21}^{0}$ e (4.30), segue do Lema 4.1.3 que

$$
\begin{aligned}
0 & =\operatorname{sen} \theta d \theta \wedge \omega_{1}^{0}-\cos \theta d \omega_{1}^{0} \\
& =\operatorname{sen} \theta\left(\theta_{1}^{0} \omega_{1}^{0}+\theta_{2}^{0} \omega_{2}^{0}\right) \wedge \omega_{1}^{0}-\cos \theta \omega_{2}^{0} \wedge\left(-A \omega_{1}^{0}-B \omega_{2}^{0}\right) \\
& =\left(\theta_{2}^{0} \operatorname{sen} \theta+A \cos \theta\right) \omega_{2}^{0} \wedge \omega_{1}^{0}
\end{aligned}
$$


isto é, $\theta_{2}^{0} \operatorname{sen} \theta+A \cos \theta=0$. Logo, de (4.32) resulta que $A=\theta_{2}^{0} \equiv 0$ e a última relação de (4.32) se reduz a

$$
B \cos \theta+2 T K_{0}=0 .
$$

Usando a terceira equação de (4.32) na primeira e na última (reescrita como acima), obtemos

$$
\begin{aligned}
& B \operatorname{sen} \theta \cos \theta+\theta_{1}^{0}=0, \\
& \theta_{2}^{0}=A \equiv 0, \\
& \operatorname{sen} \theta-2 \theta_{1}^{0} T=0, \\
& K_{0} \operatorname{sen}^{2} \theta-\left(\theta_{1}^{0}\right)^{2}=0 .
\end{aligned}
$$

Como conseqüência dessas relações, vamos mostrar que $X_{0}$ é isométrica a um parabolóide de revolução e que $l$ é uma congruência de Guichard.

Note que, de (4.34),

$$
\begin{gathered}
d \theta=\theta_{1}^{0} \omega_{1}^{0}, \\
\omega_{12}^{0}=B \omega_{2}^{0} .
\end{gathered}
$$

Como

$$
d \omega_{12}^{0}=d B \wedge \omega_{2}^{0}+B d \omega_{2}^{0}=\left(d B+B^{2} \omega_{1}^{0}\right) \wedge \omega_{2}^{0},
$$

usando a equação de Gauss, resulta

$$
\left[d B+\left(B^{2}+K_{0}\right) \omega_{1}^{0}\right] \wedge \omega_{2}^{0}=0 .
$$

Calculando, agora, a derivada exterior da equação (4.33) temos

$$
\begin{aligned}
0 & =d B \operatorname{sen} \theta \cos \theta+B\left(\cos ^{2} \theta-\operatorname{sen}^{2} \theta\right) d \theta+d \theta_{1}^{0} \\
& =d B \operatorname{sen} \theta \cos \theta-\frac{\theta_{1}^{0}}{\operatorname{sen} \theta \cos \theta}\left(\cos ^{2} \theta-\operatorname{sen}^{2} \theta\right) d \theta+d \theta_{1}^{0},
\end{aligned}
$$

de onde

$$
d B=\frac{\left(\cos ^{2} \theta-\operatorname{sen}^{2} \theta\right) \theta_{1}^{0}}{\operatorname{sen}^{2} \theta \cos ^{2} \theta} d \theta-\frac{1}{\operatorname{sen} \theta \cos \theta} d \theta_{1}^{0} .
$$

Substituindo (4.33), (4.36) e (4.38) em (4.37) obtemos

$$
\left(3 \cos \theta \theta_{1}^{0} d \theta-\operatorname{sen} \theta d \theta_{1}^{0}\right) \wedge \omega_{2}^{0}=0 .
$$

Também como $d \theta \wedge \omega_{1}^{0}=0$ e $d \theta_{1}^{0} \wedge \omega_{1}^{0}=0$, segue que

$$
\left(3 \cos \theta \theta_{1}^{0} d \theta-\operatorname{sen} \theta d \theta_{1}^{0}\right) \wedge \omega_{1}^{0}=0 .
$$

Portanto, resulta de (4.39) e (4.40) que

$$
3 \cos \theta \theta_{1}^{0} d \theta-\operatorname{sen} \theta d \theta_{1}^{0}=0,
$$

isto é,

$$
\frac{d \theta_{1}^{0}}{\theta_{1}^{0}}=3 \frac{\cos \theta}{\operatorname{sen} \theta} d \theta
$$

Integrando, temos

$$
\theta_{1}^{0}=c \operatorname{sen}^{3} \theta, \quad c \neq 0 .
$$


Logo, de (4.33) e (4.35), concluímos que

$$
B=-\frac{c \operatorname{sen}^{2} \theta}{\cos \theta}, \quad T=\frac{1}{2 c \operatorname{sen}^{2} \theta} .
$$

Não é restritivo supor que a parametrização de $X_{0}$ seja ortogonal (veja [2], p. 96), isto é, $\omega_{1}^{0}=\sqrt{E_{0}} d u$ e $\omega_{2}^{0}=\sqrt{G_{0}} d v$. Logo,

$$
\omega_{12}^{0}=B \sqrt{G_{0}} d v
$$

Assim, usando as equações de estrutura,

$$
\begin{aligned}
& d \omega_{1}^{0}=\omega_{2}^{0} \wedge \omega_{21}^{0}=\left(\sqrt{G_{0}} d v\right) \wedge\left(-B \sqrt{G_{0}} d v\right)=0, \\
& d \omega_{2}^{0}=\omega_{1}^{0} \wedge \omega_{12}^{0}=\left(\sqrt{E_{0}} d u\right) \wedge\left(B \sqrt{G_{0}} d v\right)=B \sqrt{E_{0} G_{0}} d u \wedge d v,
\end{aligned}
$$

isto é,

$$
\begin{aligned}
& \left(\sqrt{E_{0}}\right)_{v}=0, \\
& \frac{\left(\sqrt{G_{0}}\right)_{u}}{\sqrt{G_{0}}}=B \sqrt{E_{0}} .
\end{aligned}
$$

Como $E_{0}=E_{0}(u)$, por uma mudança de variável ${ }^{1}$, podemos considerar $E_{0}=1$. Então, $d \theta=$ $\theta_{1}^{0} \omega_{1}^{0}=\theta_{1}^{0} d u$, de onde resulta que $\theta_{u}=\theta_{1}^{0}$ e $\theta_{v}=0$. Assim, de (4.33), segue que

$$
\frac{\left(\sqrt{G_{0}}\right)_{u}}{\sqrt{G_{0}}}=B=-\frac{\theta_{u}}{\operatorname{sen} \theta \cos \theta}=-\left(\frac{\cos \theta}{\operatorname{sen} \theta}+\frac{\operatorname{sen} \theta}{\cos \theta}\right) \theta_{u}
$$

ou seja,

$$
\frac{\partial}{\partial u}\left(\log \sqrt{G_{0}}\right)=\frac{\partial}{\partial u}(\log |\cot \theta(u)|) .
$$

Integrando, obtemos $\sqrt{G_{0}}=f(v) \cot \theta(u)$, onde $f(v) \neq 0$ é uma função a valores reais. Novamente, por uma mudança de variável ${ }^{2}$, podemos considerar

$$
\sqrt{G_{0}}=\cot \theta(u)
$$

${ }^{1}$ Como $E_{0}$ é função somente de $u$, podemos definir uma função $p$ pondo

$$
p(u)=\int \sqrt{E_{0}(u)} d u .
$$

Como $p^{\prime}$ não se anula, pelo Teorema da Função Inversa, existe a inversa $u=u(p)$ de $p$. Definindo $Y(p, v)=$ $X_{0}(u(p), v)$, temos:

$$
E_{Y}(p)=u^{\prime}(p)^{2} E_{0}(u(p))=\frac{1}{p^{\prime}(u(p))^{2}} E_{0}(u(p))=1
$$

${ }^{2}$ Como $f=f(v)$, podemos definir uma função $q$ pondo

$$
q(v)=\int f(v) d v
$$

Como $q^{\prime}$ não se anula, pelo Teorema da Função Inversa, existe a inversa $v=v(q)$ de $q$. Definindo $Y(u, q)=$ $X_{0}(u, v(q))$, o coeficiente $G_{Y}$ da primeira forma quadrática de $Y$ é dado por:

$$
G_{Y}(u, q)=v^{\prime}(q)^{2} G_{0}(u, v(q))=\frac{1}{f(v(q))^{2}} G_{0}(u, v(q))=\cot ^{2} \theta(u) .
$$


Além disso, do Lema 4.1.3 e de (4.42), resulta que

$$
0=\cos \theta \omega_{1}^{0}+d T=\cos \theta \omega_{1}^{0}-\frac{\cos \theta}{c \operatorname{sen}^{3} \theta} d \theta
$$

e como $\cos \theta \neq 0$, obtemos

$$
\omega_{1}^{0}=\frac{1}{c \operatorname{sen}^{3} \theta} d \theta=d u
$$

Assim, a primeira forma quadrática de $X_{0}$ pode ser reescrita como

$$
\mathrm{I}_{0}=d u^{2}+\cot ^{2} \theta d v^{2}=\frac{1}{c^{2} \operatorname{sen}^{6} \theta} d \theta^{2}+\cot ^{2} \theta d v^{2} .
$$

Considerando a mudança de variáveis

$$
\left\{\begin{array}{l}
x= \pm \frac{1}{c} \cot \theta \\
y=c v
\end{array}\right.
$$

como

$$
c^{2} x^{2}+1=\frac{1}{\operatorname{sen}^{2} \theta} \quad \text { e } \quad d x=\mp \sec ^{2} \theta d \theta
$$

temos

$$
\mathrm{I}_{0}=\left(1+c^{2} x^{2}\right) d x^{2}+x^{2} d y^{2}
$$

ou seja, a métrica do parabolóide de revolução obtido pela rotação da parábola $z=\frac{c x^{2}}{2}$ em torno do eixo $z$.

Observe que

$$
\tan \theta= \pm \frac{1}{c x}
$$

e, de (4.42), temos que

$$
T=\frac{1}{2 c \operatorname{sen}^{2} \theta}=\frac{1+c^{2} x^{2}}{2 c} .
$$

Conseqüentemente, considerando $a=\frac{1}{c}$, obtemos

$$
T=\frac{a^{2}+x^{2}}{2 a}, \quad \tan \theta= \pm \frac{a}{x},
$$

que coincidem com (4.16) do Teorema 4.2.5 e com as correspondentes do Teorema 4.2.6, quando substituímos $\theta$ por $(-\theta)$. Isto termina a prova.

\subsubsection{Tratamento do Problema 4.0.5 para o caso $K>0$}

Agora vamos apresentar um resultado análogo ao anterior para o caso de superfícies de curvatura gaussiana constante positiva ou de curvatura média constante não nula.

Teorema 4.3.2. Seja $l: S_{0} \rightarrow S$ uma congruência de retas normal à superfície $S$ de curvatura gaussiana constante $K>0$ constante (respectivamente, de curvatura média constante $\mathrm{H} \neq 0$ ) $e$ tal que, para toda isometria $\tau: S_{0} \rightarrow \bar{S}_{0}$, a congruência de retas $\bar{l}$ (associada a l e $\tau$ ) é normal a uma superfície de mesma curvatura gaussiana constante $\bar{K}=K>0$ (respectivamente, de mesma curvatura média constante $\overline{\mathrm{H}}=\mathrm{H} \neq 0$ ). Se as retas determinadas por $l$ não forem tangentes a $S_{0}$, então 
(i) $S_{0}$ é isométrica a um elipsóide de revolução ou a um hiperbolóide de revolução de duas folhas;

(ii) l é uma congruência de Guichard.

Demonstração. Faremos a prova apenas no caso em que $S$ tem curvatura gaussiana constante positiva pois, pelo Teorema de Bonnet, este caso é equivalente ao de considerar $S$ de curvatura média constante não nula. Observe também que a prova é idêntica ao do teorema anterior até as fórmulas (4.31).

Suponha, inicialmente, $\bar{K}=K \neq 0$. Por hipótese, para toda superfície isométrica a $S_{0}, \bar{S}$ tem curvatura $K$. Então, de (4.30) e (4.31), temos

$$
\begin{aligned}
-K \bar{\omega}_{1} \wedge \bar{\omega}_{2}=-K[ & \operatorname{sen} \theta+T B \operatorname{sen} \theta \cos \theta-T \theta_{1}^{0}-T^{2} B \theta_{1}^{0} \cos \theta+T^{2} A \theta_{2}^{0} \cos \theta \\
& +T^{2} \mathrm{~K}_{0} \operatorname{sen} \theta-\left(T+T^{2} B \cos \theta\right) \bar{h}_{11}^{0}+\left(-T^{2} \theta_{2}^{0} \operatorname{sen} \theta+T^{2} A \cos \theta\right) \bar{h}_{12}^{0} \\
& \left.+\left(-T \operatorname{sen}^{2} \theta+T^{2} \theta_{1}^{0} \operatorname{sen} \theta\right) \bar{h}_{22}^{0}\right] \omega_{1}^{0} \wedge \omega_{2}^{0},
\end{aligned}
$$

$\mathrm{e}$

$$
\begin{aligned}
d \bar{\omega}_{12}= & {\left[B \theta_{1}^{0} \cos \theta-A \theta_{2}^{0} \cos \theta-K_{0} \operatorname{sen} \theta+B \bar{h}_{11}^{0} \cos \theta\right.} \\
& \left.+\left(\theta_{2}^{0} \operatorname{sen} \theta-A \cos \theta\right) \bar{h}_{12}^{0}-\theta_{1}^{0} \bar{h}_{22}^{0} \operatorname{sen} \theta\right] \omega_{1}^{0} \wedge \omega_{2}^{0} .
\end{aligned}
$$

Logo, segue da equação de Gauss e da independência linear de $\omega_{1}^{0}$ e $\omega_{2}^{0}$ que

$$
\begin{aligned}
& {\left[\left(T^{2} K-1\right) B \cos \theta+T K\right] \bar{h}_{11}^{0}+\left(T^{2} K-1\right)\left(\theta_{2}^{0} \operatorname{sen} \theta-A \cos \theta\right) \bar{h}_{12}^{0}} \\
& -\left[\left(T^{2} K-1\right) \theta_{1}^{0}-T K \operatorname{sen} \theta\right] \bar{h}_{22}^{0}+\left[\left(T^{2} K-1\right)\left(B \theta_{1}^{0} \cos \theta-A \theta_{2}^{0} \cos \theta-K_{0} \operatorname{sen} \theta\right)\right. \\
& \left.-K \operatorname{sen} \theta-B K T \operatorname{sen} \theta \cos \theta+\theta_{1}^{0} T K\right]=0,
\end{aligned}
$$

que deve ser satisfeita para todo $\bar{h}_{i j}^{0}, i, j=1,2$. Anulando os coeficientes de $\bar{h}_{i j}^{0}$ e o termo independente, obtemos

$$
\begin{aligned}
& \left(T^{2} K-1\right) B \cos \theta+T K=0, \\
& \left(T^{2} K-1\right)\left(\theta_{2}^{0} \operatorname{sen} \theta-A \cos \theta\right)=0, \\
& \left(T^{2} K-1\right) \theta_{1}^{0}-T K \operatorname{sen} \theta=0, \\
& \left(T^{2} K-1\right)\left(B \theta_{1}^{0} \cos \theta-A \theta_{2}^{0} \cos \theta-K_{0} \operatorname{sen} \theta\right) \\
& \quad-K \operatorname{sen} \theta-B K T \operatorname{sen} \theta \cos \theta+\theta_{1}^{0} T K=0,
\end{aligned}
$$

onde $\operatorname{sen} \theta \neq 0$, pois $l$ é uma congruência de retas não tangentes a $X_{0}$.

Note que, da primeira equação de (4.45), temos que $\cos \theta \neq 0$ e $\left(T^{2} K-1\right) \neq 0$ e, assim, a segunda equação se reduz a

$$
\theta_{2}^{0} \operatorname{sen} \theta-A \cos \theta=0 .
$$

Por outro lado, do Lema 4.1.3, segue que

$$
\theta_{2}^{0} \operatorname{sen} \theta+A \cos \theta=0
$$

de onde obtemos $A=\theta_{2}^{0} \equiv 0$. Substituindo a terceira relação de (4.45) na primeira, resulta

$$
B \operatorname{sen} \theta \cos \theta+\theta_{1}^{0}=0
$$


e, assim, da última relação de (4.45), temos

$$
T\left[\left(\theta_{1}^{0}\right)^{2}-K_{0} \operatorname{sen}^{2} \theta\right]-\theta_{1}^{0} \operatorname{sen} \theta=0 .
$$

Portanto, as (4.45) se reduzem a

$$
\begin{aligned}
& B \operatorname{sen} \theta \cos \theta+\theta_{1}^{0}=0, \\
& A=\theta_{2}^{0}=0, \\
& \left(T^{2} K-1\right) \theta_{1}^{0}-T K \operatorname{sen} \theta=0, \\
& T\left[\left(\theta_{1}^{0}\right)^{2}-K_{0} \operatorname{sen}^{2} \theta\right]-\theta_{1}^{0} \operatorname{sen} \theta=0 .
\end{aligned}
$$

Usaremos estas relações para mostrar que $X_{0}$ é isométrica a um elipsóide de revolução ou a um hiperbolóide de revolução de duas folhas e que $l$ é uma congruência de Guichard.

Novamente de (4.47) temos

$$
d \theta=\theta_{1}^{0} \omega_{1}^{0}, \quad \text { e } \quad \omega_{12}^{0}=B \omega_{2}^{0},
$$

ou seja, continuam valendo as relações (4.37) e (4.38).

Denotando $d \theta_{1}^{0}=\theta_{11}^{0} \omega_{1}^{0}+\theta_{12}^{0} \omega_{2}^{0}$, sendo $d \theta_{1}^{0} \wedge \omega_{1}^{0}=0$, segue que $\theta_{12}^{0}=0$, isto é, $d \theta_{1}^{0}=\theta_{11}^{0} \omega_{1}^{0}$. Então, substituindo (4.38), (4.46) e (4.49) em (4.37), temos

$$
\begin{aligned}
0= & {\left[d B+\left(B^{2}+K_{0}\right) \omega_{1}^{0}\right] \wedge \omega_{2}^{0} } \\
= & {\left[\frac{\left(\cos ^{2} \theta-\operatorname{sen}^{2} \theta\right) \theta_{1}^{0}}{\operatorname{sen}^{2} \theta \cos ^{2} \theta} d \theta-\frac{1}{\operatorname{sen} \theta \cos \theta} d \theta_{1}^{0}\right.} \\
& \left.+\left(\frac{\left.\theta_{1}^{0}\right)^{2}}{\operatorname{sen}^{2} \theta \cos ^{2} \theta}+\frac{T\left(\theta_{1}^{0}\right)^{2}-\theta_{1}^{0} \operatorname{sen} \theta}{T \operatorname{sen}^{2} \theta}\right) \omega_{1}^{0}\right] \wedge \omega_{2}^{0} \\
= & \frac{3 T\left(\theta_{1}^{0}\right)^{2} \cos \theta-T \theta_{11}^{0} \operatorname{sen} \theta-\theta_{1}^{0} \operatorname{sen} \theta \cos \theta}{T \operatorname{sen}^{2} \theta \cos \theta} \omega_{1}^{0} \wedge \omega_{2}^{0},
\end{aligned}
$$

ou seja,

$$
T\left[3\left(\theta_{1}^{0}\right)^{2} \cos \theta-\theta_{11}^{0} \operatorname{sen} \theta\right]=\theta_{1}^{0} \operatorname{sen} \theta \cos \theta
$$

Portanto

$$
d \log \left|3\left(\theta_{1}^{0}\right)^{2} \cos \theta-\theta_{11}^{0} \operatorname{sen} \theta\right|=d \log \left|\theta_{1}^{0} \operatorname{sen} \theta \cos \theta\right|+\frac{3\left(\theta_{1}^{0}\right)^{2} \cos \theta-\theta_{11}^{0} \operatorname{sen} \theta}{\theta_{1}^{0} \operatorname{sen} \theta} \omega_{1}^{0} .
$$

Como $d \theta_{1}^{0}=\theta_{11}^{0} \omega_{1}^{0}$ e $d \theta=\theta_{1}^{0} \omega_{1}^{0}$, segue

$$
\begin{aligned}
& d \log \left|\theta_{1}^{0} \operatorname{sen} \theta \cos \theta\right|+\frac{3\left(\theta_{1}^{0}\right)^{2} \cos \theta-\theta_{11}^{0} \operatorname{sen} \theta}{\theta_{1}^{0} \operatorname{sen} \theta} \omega_{1}^{0} \\
& =\frac{1}{\theta_{1}^{0}} d \theta_{1}^{0}+\left(\frac{\cos \theta}{\operatorname{sen} \theta}-\frac{\operatorname{sen} \theta}{\cos \theta}\right) d \theta+\frac{3\left(\theta_{1}^{0}\right)^{2} \cos \theta-\theta_{11}^{0} \operatorname{sen} \theta}{\theta_{1}^{0} \operatorname{sen} \theta} \omega_{1}^{0}, \\
& =\frac{4 \cos ^{2} \theta-\operatorname{sen}^{2} \theta}{\operatorname{sen} \theta \cos \theta} d \theta=\frac{4-5 \operatorname{sen}^{2} \theta}{\operatorname{sen} \theta \cos \theta} d \theta \\
& =d \log \left|\operatorname{sen}^{4} \theta \cos \theta\right|,
\end{aligned}
$$

de onde resulta que

$$
d \log \left|3\left(\theta_{1}^{0}\right)^{2} \cos \theta-\theta_{11}^{0} \operatorname{sen} \theta\right|=d \log \left|\operatorname{sen}^{4} \theta \cos \theta\right| .
$$


Integrando, obtemos

$$
3\left(\theta_{1}^{0}\right)^{2} \cos \theta-\theta_{11}^{0} \operatorname{sen} \theta=c \operatorname{sen}^{4} \theta \cos \theta, \quad c \neq 0
$$

e, de (4.50), segue que

$$
T=\frac{\theta_{1}^{0}}{c \operatorname{sen}^{3} \theta}
$$

Substituindo (4.52) em (4.48), temos

$$
\left(\theta_{1}^{0}\right)^{2}=c \operatorname{sen}^{4} \theta+\frac{c^{2}}{K} \operatorname{sen}^{6} \theta .
$$

Assim, de (4.52) resulta

$$
T^{2}=\frac{1}{K}+\frac{1}{c \operatorname{sen}^{2} \theta} .
$$

Não é restritivo supor que $X_{0}$ seja uma parametrização ortogonal (veja [2], p. 96), ou seja, $\omega_{1}^{0}=\sqrt{E_{0}} d u, \omega_{2}^{0}=\sqrt{G_{0}} d v$ e, assim, $\omega_{12}^{0}=B \sqrt{G_{0}} d v$. Procedendo como na demonstração do Teorema 4.3.1, obtemos

$$
\left(\sqrt{E_{0}}\right)_{v}=0, \quad \frac{\left(\sqrt{G_{0}}\right)_{u}}{\sqrt{G_{0}}}=B \sqrt{E_{0}}
$$

Novamente, por uma mudança de variável, podemos considerar $E_{0}=1 \mathrm{e}$, portanto, $d \theta=\theta_{1}^{0} d u=$ $\theta_{u} d u$. Segue de (4.46) que

$$
\frac{\left(\sqrt{G_{0}}\right)_{u}}{\sqrt{G_{0}}}=-\frac{\theta_{u}}{\cos \theta \operatorname{sen} \theta}
$$

de onde concluímos que $\sqrt{G_{0}}=f(v) \cot \theta(u)$. Também por uma mudança de variável, podemos considerar

$$
\sqrt{G_{0}}=\cot \theta
$$

Note que, como $d \theta=\theta_{1}^{0} d u$, de (4.53) temos que

$$
\mathrm{I}_{0}=E_{0} d u^{2}+G_{0} d v^{2}=\frac{1}{c \operatorname{sen}^{4} \theta+\frac{c^{2}}{K} \operatorname{sen}^{6} \theta} d \theta^{2}+\cot ^{2} \theta d v^{2}
$$

Queremos reescrever $\mathrm{I}_{0}$ na forma:

$$
\mathrm{I}_{0}=\left(1+\psi^{\prime}(x)^{2}\right) d x^{2}+x^{2} d y^{2} .
$$

Para isso, consideramos a seguinte mudança de variáveis

$$
\left\{\begin{array}{l}
x=a \cot \theta, \quad(\text { respectivamente, } \quad x=-a \cot \theta) \\
y=\frac{v}{a},
\end{array}\right.
$$

onde $a$ é uma constante não nula e $\psi(x)$ uma função tal que

$$
\left(1+\psi^{\prime}(x)^{2}\right) d x^{2}=\frac{1}{c \operatorname{sen}^{4} \theta\left(1+\frac{c}{K} \operatorname{sen}^{2} \theta\right)} d \theta^{2} .
$$


Como $x=a \cot \theta$, temos que

$$
\operatorname{sen}^{2} \theta=\frac{a^{2}}{a^{2}+x^{2}}
$$

e

$$
\psi^{\prime}(x)^{2}=\frac{x^{2}\left(1-a^{2} c\right)+a^{2}\left(1-a^{2} c-\frac{c^{2}}{K} a^{2}\right)}{a^{2} c\left(a^{2}+x^{2}+\frac{c}{K} a^{2}\right)} .
$$

A partir daqui vamos supor que $K>0$. Como $\theta_{1}^{0} \neq 0$, de (4.53) segue que

$$
0<\frac{\left(\theta_{1}^{0}\right)^{2}}{\operatorname{sen}^{4} \theta}=c+\frac{c^{2}}{K} \operatorname{sen}^{2} \theta=\left(c+\frac{c^{2}}{K}\right)-\frac{c^{2}}{K} \cos ^{2} \theta,
$$

isto é,

$$
\frac{c(K+c)}{K}>0
$$

Então, escolha $a$ tal que

$$
a^{2}=\frac{K}{c(c+K)}
$$

Note que

$$
1-a^{2} c-a^{2} \frac{c^{2}}{K}=0
$$

e, portanto, (4.58) se reduz a

$$
\psi^{\prime}(x)=\frac{x c}{\sqrt{K\left(x^{2} c+1\right)}}, \quad \quad x^{2} c+1>0,
$$

de onde obtemos, a menos de uma constante, que $\psi(x)=\sqrt{\frac{x^{2} c+1}{K}}$. Logo,

$$
\mathrm{I}_{0}=\left(1+\psi^{\prime}(x)^{2}\right) d x^{2}+x^{2} d y^{2}
$$

é a métrica da superfície de revolução (ao redor do eixo $z$ ) cuja curva geradora $\alpha(x)=(x, 0, \psi(x))$ é tal que

$$
K \psi(x)^{2}-c x^{2}=1 .
$$

Conseqüentemente:

- se $c>0$, então $\alpha(x)$ é a hipérbole:

$$
\frac{\psi(x)^{2}}{\left(\frac{1}{\sqrt{K}}\right)^{2}}-\frac{x^{2}}{\left(\frac{1}{\sqrt{c}}\right)^{2}}=1,
$$

cujo eixo transverso, de comprimento $\frac{2}{\sqrt{K}}$, coincide com o eixo de rotação.

- se $c<0$, de (4.59) temos que $\frac{1}{\sqrt{K}}>\frac{1}{\sqrt{-c}}$ e a curva $\alpha(x)$ é a elipse:

$$
\frac{\psi(x)^{2}}{\left(\frac{1}{\sqrt{K}}\right)^{2}}+\frac{x^{2}}{\left(\frac{1}{\sqrt{-c}}\right)^{2}}=1,
$$


cujo eixo maior, de comprimento $\frac{2}{\sqrt{K}}$, coincide com o eixo $z$. Neste caso, de (4.56), resulta que

$$
\left.\tan \theta=\frac{a}{x}=\frac{\sqrt{K}}{x \sqrt{c(c+K)}}, \quad \text { (respectivamente, } \quad \tan \theta=-\frac{a}{x}=\frac{-\sqrt{K}}{x \sqrt{c(c+K)}}\right),
$$

e, de (4.54), temos (para $T>0)$

$$
T=\sqrt{\frac{c+K}{c}} \psi(x)
$$

e, portanto,

$$
\tilde{T}=T+\frac{1}{\sqrt{K}}=\frac{1}{\sqrt{K}}+\sqrt{\frac{c+K}{c}} \psi(x) .
$$

Note que $\theta$ e $\tilde{T}$, assim definidos, são equivalentes às equações (4.26) do Teorema 4.2.8 para o caso no qual o foco do elipsóide de revolução é $\left(0,0,-\sqrt{\frac{c+K}{c K}}\right)$, o que nos permite concluir que $l$ é uma congruência de Guichard.

\subsubsection{Tratamento do Problema 4.0.5 para o caso $K<0$}

Observe que, na prova do Teorema 4.3.2 usamos a hipótese da superfície $S$ possuir curvatura gaussiana $K$ constante positiva somente após obtermos a equação (4.58). Assim, podemos pensar em um caso análogo a este, mas considerando uma superfície $S$ pseudoesférica. Para isso, definiremos três superfícies de revolução que, junto às da Definição 4.2.4, são chamadas por Bianchi de superfícies fundamentais de revolução.

Definição 4.3.3. $\quad$ (i) A superfície logarítmica de revolução é obtida pela rotação da curva $z=\log x$ ao redor do eixo $z$.

(ii) O catenóide contraído é obtido pela rotação da curva $x=\lambda \frac{\cosh (a z)}{a}$, onde $a \neq 0$ e $0<\lambda<1$ são constantes, em torno do eixo $z$.

(iii) O senusóide hiperbólico é obtido pela rotação do senóide hiperbólico $x=\lambda \operatorname{senh} z$, onde $\lambda>0$ é constante, ao redor do eixo $z$.

Teorema 4.3.4. Seja $l: S_{0} \rightarrow S$ uma congruência de retas normal a uma superfície $S$ de curvatura gaussiana $K<0$ constante e tal que, para toda isometria $\tau: S_{0} \rightarrow \bar{S}_{0}$, a congruência de retas $\bar{l}$ (associada a $l$ e $\tau$ ) é normal a uma superfície $\bar{S}$ de mesma curvatura gaussiana $\bar{K}=K<0$ constante. Se as retas determinadas por l não forem tangentes a $S_{0}$, então $S_{0} e ́$ isométrica a uma das três superfícies de rotação da Definição 4.3.3.

Demonstração. Esta demonstração segue análoga a do Teorema 4.3.2 até obtermos a equação (4.58). Como $K<0$, da equação (4.53):

$$
0<\left(\theta_{1}^{0}\right)^{2}=c \operatorname{sen}^{4} \theta+\frac{c^{2}}{K} \operatorname{sen}^{6} \theta
$$


segue que $c>0$. Escolhendo, então, a constante $a$ tal que

$$
a^{2}=\frac{1}{c}
$$

a equação (4.58) se reduz a

$$
\psi^{\prime}(x)=\frac{1}{\sqrt{-K} \sqrt{x^{2}+\frac{1}{c}+\frac{1}{K}}} .
$$

Denotando, agora, $\lambda=\frac{1}{c}+\frac{1}{K}$, então temos os casos listados a seguir.

(i) Se $\lambda=0$, segue de (4.60) que a curva geradora $\alpha(x)=(x, 0, \psi(x))$ é a curva logarítmica:

$$
\sqrt{-K} \psi(x)=\log x
$$

isto é, $S_{0}$ é isométrica à superfície logarítmica. Neste caso, de (4.56), resulta que

$$
\tan \theta=\frac{1}{\sqrt{-K} x}
$$

e, de (4.54),

$$
T=\frac{\cot \theta}{\sqrt{-K}}
$$

(ii) Se $\lambda<0$, de (4.60) segue que

$$
\sqrt{-K} \psi^{\prime}(x)=\frac{1}{\sqrt{-\lambda} \sqrt{\left(\frac{x}{\sqrt{-\lambda}}\right)^{2}-1}}
$$

de onde obtemos que $x=\sqrt{\lambda K} \frac{\cosh (\sqrt{-K} \psi(x))}{\sqrt{-K}}$, sendo $\lambda K<1$, isto é, $S_{0}$ é isométrica ao catenóide contraído.

(iii) Se $\lambda>0$, de (4.60) temos que

$$
\sqrt{-K} \psi^{\prime}(x)=\frac{1}{\sqrt{\lambda} \sqrt{\left(\frac{x}{\sqrt{\lambda}}\right)^{2}+1}} .
$$

Integrando, obtemos $x=\sqrt{\lambda} \operatorname{senh}(\sqrt{-K} \psi(x))$, ou seja, $S_{0}$ é isométrica ao senusóide hiperbólico.

Nesses dois últimos casos, resulta que

$$
\tan \theta=\frac{1}{\sqrt{c} x}
$$

$\mathrm{e}$

$$
T^{2}=\frac{1}{K}+\frac{1}{c \operatorname{sen}^{2} \theta}
$$


As condições necessárias obtidas no Teorema 4.3.4 também são suficientes para a existência da congruência de retas satisfazendo este teorema. Mais precisamente, vale o seguinte:

Teorema 4.3.5. Seja $X_{0}(u, v),(u, v) \in U \subset \mathbb{R}^{2}$, a parametrização de uma das superfícies da Definição 4.3.3 e $\bar{X}_{0}=\tau\left(X_{0}\right)$, onde $\tau$ é uma isometria. Considere o triedro móvel associado a $\bar{X}_{0}$ dado por

$$
\bar{e}_{i}^{0}=d \tau\left(e_{i}^{0}\right), \quad i=1,2 ; \quad \bar{e}_{3}^{0}=\bar{e}_{1}^{0} \wedge \bar{e}_{2}^{0},
$$

onde $e_{1}^{0}, e_{2}^{0}$ são campos de vetores tangentes aos meridianos e aos paralelos de $X_{0}$, respectivamente. Suponha também que $\theta, T$ sejam definidas pelas equações (4.61) e (4.62), quando $X_{0}$ é a superfície logarítmica, e pelas equações (4.63) e (4.64) nos outros dois casos. Então, as congruências de retas:

$$
\bar{X}=\bar{X}_{0}+T\left(\cos \theta \bar{e}_{1}^{0}+\operatorname{sen} \theta \bar{e}_{3}^{0}\right), \quad \bar{X}^{s}=\bar{X}_{0}+T\left(\cos \theta \bar{e}_{1}^{0}-\operatorname{sen} \theta \bar{e}_{3}^{0}\right),
$$

são normais (respectivamente) às superfícies $\bar{X}$ e $\bar{X}^{s}$ de mesma curvatura gaussiana constante negativa.

Devido à semelhança dos resultados obtidos para as superfícies da Definição $4.3 .3 \mathrm{com}$ as outras superfícies de revolução, a saber o parabolóide de revolução, dadas na Definição 4.2.4, temos a seguinte:

Definição 4.3.6. As congruências de retas

$$
\bar{X}=\bar{X}_{0}+T\left(\cos \theta \bar{e}_{1}^{0}+\operatorname{sen} \theta \bar{e}_{3}^{0}\right), \quad \bar{X}^{s}=\bar{X}_{0}+T\left(\cos \theta \bar{e}_{1}^{0}-\operatorname{sen} \theta \bar{e}_{3}^{0}\right),
$$

obtidas no Teorema 4.3.5, onde $\bar{X}_{0}$ é isométrica a uma das superfícies da Definição 4.2.4, são denominadas por congruências de Guichard, quando as funções $T$ e $\theta$ satisfazem às seguintes relações:

\begin{tabular}{|c||c|c|}
\hline & $T$ & $\tan \theta$ \\
\hline \hline $\begin{array}{c}\text { Superfície logarítmica } \\
\text { de revolução }\end{array}$ & $\frac{\cot \theta}{\sqrt{-K}}$ & $\frac{1}{\sqrt{-K} x}$ \\
\hline $\begin{array}{c}\text { Catenóide contraido } \\
\text { ou }\end{array}$ & $\pm \sqrt{\frac{1}{K}+\frac{1}{c \operatorname{sen}^{2} \theta}}$ & $\frac{a^{2}}{c x}, c>0$ \\
senusóide hiperbólico & & \\
\hline
\end{tabular}

Das equações (4.42) e (4.54) obtidas, respectivamente, nos Teoremas 4.3 .1 e 4.3.2, seguem os resultados:

Corolário 4.3.7. Se $X=X_{0}+T\left(\cos \theta e_{1}^{0}+\operatorname{sen} \theta e_{3}^{0}\right)$ for uma congruência de Guichard, sendo $X$ uma superfície mínima, então

$$
T=\frac{1}{2 c \operatorname{sen}^{2} \theta},
$$

onde c é uma constante não nula.

Corolário 4.3.8. Se $X=X_{0}+T\left(\cos \theta e_{1}^{0}+\operatorname{sen} \theta e_{3}^{0}\right)$ for uma congruência de Guichard, onde $X$ é uma superfície de curvatura gaussiana constante $K$ não nula. Então

$$
T^{2}=\frac{1}{K}+\frac{1}{c \operatorname{sen}^{2} \theta},
$$

onde c é uma constante positiva se $K<0$ e não nula se $K>0$. 


\subsubsection{Algumas propriedades das congruências de Guichard}

Como últimos resultados deste capítulo, vamos apresentar algumas propriedades das congruências de Guichard provadas por Bianchi em [6], §7,8,9. Consideramos, inicialmente, superfícies isométricas a um parabolóide de revolução.

Proposição 4.3.9. Se $l: S_{0} \rightarrow S$ é uma congruência de Guichard com $S_{0}$ isométrica a um parabolóide de revolução, então direções conjugadas de $S_{0}$ são levadas em direções ortogonais de $S$.

Demonstração. Sejam $X_{0}, X: U \subset \mathbb{R}^{2} \rightarrow \mathbb{R}^{3}$ parametrizações das superfícies $S$ e $S_{0}$, respectivamente, e $\left\{e_{1}^{0}, e_{2}^{0}, e_{3}^{0}\right\}$ um triedro móvel associado a $X_{0}$. Se a congruência de Guichard $l$ for dada por:

$$
X=X_{0}+T\left(\cos \theta e_{1}^{0}+\operatorname{sen} \theta e_{3}^{0}\right),
$$

podemos considerar, associado a $X$, o seguinte triedro móvel dado como na Observação 4.1.4:

$$
\begin{aligned}
& e_{1}=\operatorname{sen} \theta e_{1}^{0}-\cos \theta e_{3}^{0}, \\
& e_{2}=e_{2}^{0}, \\
& e_{3}=\cos \theta e_{1}^{0}+\operatorname{sen} \theta e_{3}^{0}
\end{aligned}
$$

e as 1-formas associadas a este triedro:

$$
\begin{aligned}
\omega_{1} & =\operatorname{sen} \theta \omega_{1}^{0}-T d \theta-T \omega_{13}^{0}, \\
\omega_{2} & =\omega_{2}^{0}+T \cos \theta \omega_{12}^{0}-T \operatorname{sen} \theta \omega_{23}^{0}, \\
\omega_{13} & =d \theta+\omega_{13}^{0}, \\
\omega_{23} & =-\cos \theta \omega_{12}^{0}+\operatorname{sen} \theta \omega_{23}^{0} .
\end{aligned}
$$

Considerando os campos de vetores $V_{1}, V_{2}: U \subset \mathbb{R}^{2} \rightarrow \mathbb{R}^{2}$, de (4.32) e (4.65) resulta que

$$
\begin{aligned}
\mathrm{I}\left(V_{1}, V_{2}\right)= & \left(\omega_{1}^{2}+\omega_{2}^{2}\right)\left(V_{1}, V_{2}\right) \\
= & {\left[\operatorname{sen} \theta \omega_{1}-T d \theta-T \omega_{13}^{0}\right]^{2}\left(V_{1}, V_{2}\right)+\left[\omega_{2}^{0}+T \cos \theta \omega_{12}^{0}-T \operatorname{sen} \theta \omega_{23}^{0}\right]^{2}\left(V_{1}, V_{2}\right) } \\
= & -\frac{T}{2} \operatorname{sen} \theta \omega_{1}^{0}\left(V_{1}\right) \omega_{13}^{0}\left(V_{2}\right)+\frac{1}{4} \operatorname{sen}^{2} \theta \omega_{1}^{0}\left(V_{1}\right) \omega_{1}^{0}\left(V_{2}\right)-\frac{T}{2} \operatorname{sen} \theta \omega_{13}^{0}\left(V_{1}\right) \omega_{1}^{0}\left(V_{2}\right) \\
& +T^{2} \omega_{13}^{0}\left(V_{1}\right) \omega_{13}^{0}\left(V_{2}\right)-T \operatorname{sen} \theta[1+T B \cos \theta] \omega_{2}^{0}\left(V_{1}\right) \omega_{23}^{0}\left(V_{2}\right) \\
& +T^{2} B^{2} \cos ^{2} \theta \omega_{2}^{0}\left(V_{1}\right) \omega_{2}^{0}\left(V_{2}\right)-T \operatorname{sen} \theta \omega_{23}^{0}\left(V_{1}\right) \omega_{2}^{0}\left(V_{2}\right) \\
& -T^{2} B \operatorname{sen} \theta \cos \theta \omega_{23}^{0}\left(V_{1}\right) \omega_{2}^{0}\left(V_{2}\right)+T^{2} \operatorname{sen}^{2} \theta \omega_{23}^{0}\left(V_{1}\right) \omega_{23}^{0}\left(V_{2}\right) \\
= & \left(-T \operatorname{sen} \theta+T^{2} h_{11}^{0}+T^{2} \operatorname{sen}^{2} \theta h_{22}^{0}\right) \mathrm{II}^{0}\left(V_{1}, V_{2}\right),
\end{aligned}
$$

o que termina a prova.

Considerando, agora, superfícies isométricas a um elipsóide de revolução ou a um hiperbolóide de revolução de duas folhas temos a seguinte:

Proposição 4.3.10. Se l $: S_{0} \rightarrow S$ é uma congruência de Guichard, sendo $S_{0}$ é isométrica a um elipsóide de revolução ou a um hiperbolóide de revolução de duas folhas, então l preserva direções conjugadas. Em particular, l preserva direções assintóticas. 
Demonstração. Sejam $X_{0}, X: U \subset \mathbb{R}^{2} \rightarrow \mathbb{R}^{3}$ parametrizações de $S_{0}$ e $S$, respectivamente, $\left\{e_{1}^{0}, e_{2}^{0}, e_{3}^{0}\right\}$ um triedro móvel associado a $X_{0}$ tal que a congruência $l$ seja dada por:

$$
X=X_{0}+T\left(\cos \theta e_{1}^{0}+\operatorname{sen} \theta e_{3}^{0}\right)
$$

e $\omega_{1}^{0}, \omega_{2}^{0}, \omega_{i j}^{0}, i, j=1,2,3$, as 1 -formas associadas a este triedro móvel. Como $X_{0}$ é isométrica a um elipsóide de revolução ou a um hiperbolóide de revolução de duas folhas, resulta do II Teorema de Guichard que $X$ é uma superfície de curvatura gaussiana constante $K>0$ (ou de curvatura média $\mathrm{H} \neq 0$ ).

Considere $\left\{e_{1}, e_{2}, e_{3}\right\}$ um triedro móvel associado a $X$ dado como na Observação 4.1 .4 e, assim, as 1-formas associadas a este triedro são dadas por (4.31). Então, usando a notação (4.30), as relações de (4.46) a (4.49) estão satisfeitas. Em particular,

$$
\begin{aligned}
d \theta & =\theta_{1}^{0} \omega_{1}^{0}, \\
\omega_{12}^{0} & =B \omega_{2}^{0} .
\end{aligned}
$$

Para mostrar que $l$ preserva direções conjugadas, vamos mostrar que a segunda forma quadrática II de $X$ é proporcional a $\mathrm{II}^{0}$, segunda forma quadrática de $X_{0}$. Assim, usando as relações citadas acima, temos

$$
\begin{aligned}
\mathrm{II}= & \omega_{1} \omega_{13}+\omega_{2} \omega_{23} \\
= & {\left[\left(\operatorname{sen} \theta-T \theta_{1}^{0}\right) \omega_{1}^{0}-T \omega_{13}^{0}\right]\left(\theta_{1}^{0} \omega_{1}^{0}+\omega_{13}^{0}\right) } \\
& +\left[(1+T B \cos \theta) \omega_{2}^{0}-T \operatorname{sen} \theta \omega_{23}^{0}\right]\left(-\cos \theta B \omega_{2}^{0}+\operatorname{sen} \theta \omega_{23}^{0}\right) \\
= & {\left[\theta_{1}^{0} \operatorname{sen} \theta+h_{11}^{0} \operatorname{sen} \theta-T\left(\theta_{1}^{0}\right)^{2}-2 T \theta_{1}^{0} h_{11}^{0}-T\left(h_{11}^{0}\right)^{2}-T \operatorname{sen}^{2} \theta\left(h_{12}^{0}\right)^{2}\right]\left(\omega_{1}^{0}\right)^{2} } \\
& +\left[\operatorname{sen} \theta h_{12}^{0}-T \theta_{1}^{0} h_{12}^{0}-T h_{11}^{0} h_{12}^{0}+T B \operatorname{sen} \theta \cos \theta h_{12}^{0}-T \operatorname{sen}^{2} \theta h_{12}^{0} h_{22}^{0}\right]\left(\omega_{1}^{0} \omega_{2}^{0}+\omega_{2}^{0} \omega_{1}^{0}\right) \\
& +\left[-T\left(h_{12}^{0}\right)^{2}-B \cos \theta+h_{22}^{0} \operatorname{sen} \theta-T B^{2} \cos ^{2} \theta+2 T B \operatorname{sen} \theta \cos \theta h_{22}^{0}-T \operatorname{sen}^{2} \theta\left(h_{22}^{0}\right)^{2}\right]\left(\omega_{2}^{0}\right)^{2} .
\end{aligned}
$$

Então, como $K_{0}=h_{11}^{0} h_{22}^{0}-\left(h_{12}^{0}\right)^{2}$, segue que

$$
\begin{aligned}
\mathrm{II} & =\left(\operatorname{sen} \theta-2 T \theta_{1}^{0}-T h_{11}^{0}-T h_{22}^{0} \operatorname{sen}^{2} \theta\right)\left[h_{11}^{0}\left(\omega_{1}^{0}\right)^{2}+h_{12}^{0}\left(\omega_{1}^{0} \omega_{2}^{0}+\omega_{2}^{0} \omega_{1}^{0}\right)+h_{22}^{0}\left(\omega_{2}^{0}\right)^{2}\right] \\
& =\left(\operatorname{sen} \theta-2 T \theta_{1}^{0}-T h_{11}^{0}-T h_{22}^{0} \operatorname{sen}^{2} \theta\right) \mathrm{II}^{0} .
\end{aligned}
$$

Portanto, $l$ preserva direções conjugadas e, conseqüentemente, preserva direções assintóticas.

Proposição 4.3.11. Sejam l $: S_{0} \rightarrow S$ e $l^{s}: S_{0} \rightarrow S^{s}$ congruências de Guichard tais que $S$ e $S^{s}$ são simétricas em relação a $S_{0}$. Então, a aplicação $l^{s} \circ l^{-1}$ preserva direções principais.

Demonstração. Sejam $X_{0}, X, X^{s}: U \subset \mathbb{R}^{2} \rightarrow \mathbb{R}^{3}$ parametrizações das superfícies $S_{0}, S$ e $S^{s}$, respectivamente. Considere $\left\{e_{1}^{0}, e_{2}^{0}, e_{3}^{0}\right\}$ um triedro móvel associado a $X_{0}$ tal que as congruências $l$ e $l^{s}$ sejam dadas por:

$$
X=X_{0}+T\left(\cos \theta e_{1}^{0}+\operatorname{sen} \theta e_{3}^{0}\right), \quad \quad X^{s}=X_{0}+T\left(\cos \theta e_{1}^{0}-\operatorname{sen} \theta e_{3}^{0}\right),
$$

e $\omega_{1}^{0}, \omega_{2}^{0}, \omega_{i j}^{0}, i, j=1,2,3$, as 1 -formas associadas a este triedro.

Considere $\left\{e_{1}, e_{2}, e_{3}\right\}$ e $\left\{e_{1}^{s}, e_{2}^{s}, e_{3}^{s}\right\}$ triedros móveis associados a $X$ e $X^{s}$, respectivamente, dados como na Observação 4.1.4. Como a congruência $l^{s}$ é obtida ao substituirmos $\theta$ por $(-\theta)$ 
em $l$, então, as 1-formas associadas a estes triedros são (respectivamente) dadas por:

$$
\begin{array}{rlrl}
\omega_{1} & =\operatorname{sen} \theta \omega_{1}^{0}-T d \theta-T \omega_{13}^{0}, & \omega_{1}^{s}=-\operatorname{sen} \theta \omega_{1}^{0}+T d \theta-T \omega_{13}^{0}, \\
\omega_{2}=\omega_{2}^{0}+T \cos \theta \omega_{12}^{0}-T \operatorname{sen} \theta \omega_{23}^{0}, & & \omega_{2}^{s}=\omega_{2}^{0}+T \cos \theta \omega_{12}^{0}+T \operatorname{sen} \theta \omega_{23}^{0}, \\
\omega_{13} & =d \theta+\omega_{13}^{0}, & & \omega_{13}^{s}=-d \theta+\omega_{13}^{0}, \\
\omega_{23} & =-\cos \theta \omega_{12}^{0}+\operatorname{sen} \theta \omega_{23}^{0}, & & \omega_{23}^{s}=-\cos \theta \omega_{12}^{0}-\operatorname{sen} \theta \omega_{23}^{0} .
\end{array}
$$

Note que, como as relações

$$
\begin{aligned}
& d \theta=\theta_{1}^{0} \omega_{1}^{0}, \\
& \omega_{12}^{0}=B \omega_{2}^{0}, \\
& B \operatorname{sen} \theta \cos \theta+\theta_{1}^{0}=0
\end{aligned}
$$

são satisfeitas para toda congruência de Guichard, temos que

$$
\begin{aligned}
\left(\omega_{1}\right)_{q}(v)\left(\omega_{23}\right)_{q}(v)-\left(\omega_{2}\right)_{q}(v)\left(\omega_{13}\right)_{q}(v) & =\operatorname{sen}^{2} \theta(q)\left(\omega_{1}^{0}\right)_{q}(v)\left(\omega_{23}^{0}\right)_{q}(v)-\left(\omega_{2}^{0}\right)_{q}(v)\left(\omega_{13}^{0}\right)_{q}(v) \\
& =\left(\omega_{1}^{s}\right)_{q}(v)\left(\omega_{23}^{s}\right)_{q}(v)-\left(\omega_{2}^{s}\right)_{q}(v)\left(\omega_{13}^{s}\right)_{q}(v),
\end{aligned}
$$

para todo $q \in U$ e $v \in \mathbb{R}^{2}$. Portanto, da Proposição 1.3.1 segue que $l^{s} \circ l^{-1}$ preserva direções principais. 


\section{Capítulo 5}

\section{Transformações de superfícies de curvatura constante}

No Capítulo 4 (Corolários 4.2 .7 e 4.2.11) vimos que a partir de uma dada superfície $S_{0}$, isométrica a uma das seis superfícies de revolução fundamentais, se deduziam um par de superfícies $S$ e $S^{s}$ (simétricas com respeito a $S_{0}$ ) e ligadas a $S_{0}$ por uma congruência de Guichard. Mais ainda, tais superfícies $S$ e $S^{s}$ resultavam mínimas no caso de $S_{0}$ ser isométrica ao parabolóide de revolução, e de mesma curvatura constante nos restantes cinco casos.

Estamos agora interessados no problema inverso, que foi proposto e resolvido por L. Bianchi em [6] e que pode ser formulado como segue:

Problema 5.0.12. Dada uma superfície $S$ de curvatura média (ou gaussiana não nula) constante, podemos sempre obtê-la por uma congruência de Guichard?

Provaremos que a resposta a esta questão é sempre afirmativa; mais que isto: a superfície $S$ pode ser obtida por uma família a três parâmetros de congruências de Guichard $l: S_{0} \rightarrow S$. Como, fixada uma tal congruência (isto é, fixada a superfície $S_{0}$ ), a superfície $S^{s}$ (simétrica de $S$ com respeito a $S_{0}$ ) é de mesma curvatura média (ou gaussiana não nula) constante, teremos assim transformações entre estes tipos de superfícies (veja Corolários 5.2.4 e 5.3.4).

No fim do capítulo estas novas transformações de superfícies serão interpretadas analiticamente através dos resultados estudados no Capítulo 2.

\subsection{Considerações preliminares e funções associadas a $T$}

Seja $X: U \subset \mathbb{R}^{2} \rightarrow \mathbb{R}^{3}$ a parametrização principal de uma superfície mínima ou de curvatura gaussiana constante não nula, $\left\{e_{1}, e_{2}, e_{3}\right\}$ um triedro móvel principal e $\omega_{1}, \omega_{2}, \omega_{i j}, i, j=1,2,3$, as 1-formas associadas a este triedro. Suponha que $X$ seja obtida a partir de uma congruência de Guichard. Então, como esta congruência é normal a $X$, segue dos Teoremas 4.3.1, 4.3.2 e 4.3.4, que $X_{0}$ dada por:

$$
X_{0}=X+T e_{3}
$$

é isométrica a uma das superfícies fundamentais, a saber: parabolóide de revolução, elipsóide de revolução, hiperbolóide de revolução de duas folhas, superfície logarítmica, catenóide contraído ou senusóide hiperbólico. 
Seja $e_{3}^{0}$ um campo unitário normal a $X_{0}$ e $\theta \in\left(0, \frac{\pi}{2}\right)$ tal que

$$
\left\langle e_{3}, e_{3}^{0}\right\rangle=\operatorname{sen} \theta
$$

Dos Corolários 4.3.7 e 4.3.8, as funções $T$ e $\theta$ a valores reais estão relacionados por:

$$
T=\frac{1}{2 c \operatorname{sen}^{2} \theta}, \quad c \neq 0
$$

se $X$ for uma superfície mínima, e por

$$
T^{2}=\frac{1}{K}+\frac{1}{c \operatorname{sen}^{2} \theta}, \quad c \neq 0,
$$

se $X$ for de curvatura gaussiana constante $K \neq 0$. Note que, conhecendo $T$, a superfície $X_{0}$ fica determinada. Além disso, das Proposições 4.3.9 e 4.3.10, congruências de Guichard levam direções principais de $X$ em direções conjugadas de $X_{0}$. Impondo esta condição, vamos obter um sistema diferencial que deve ser satisfeito por $T$.

Sendo $\kappa_{1}$ e $\kappa_{2}$ as curvaturas principais de $X$, onde $e_{1}, e_{2}$ são campos de direções principais, temos

$$
\omega_{13}=\kappa_{1} \omega_{1}, \quad \omega_{23}=\kappa_{2} \omega_{2}
$$

Assim, denotando por

$$
d T=T_{1} \omega_{1}+T_{2} \omega_{2}
$$

segue de (5.1) que

$$
d X_{0}=\left(1-T \kappa_{1}\right) \omega_{1} e_{1}+\left(1-T \kappa_{2}\right) \omega_{2} e_{2}+\left(T_{1} \omega_{1}+T_{2} \omega_{2}\right) e_{3} .
$$

Observe que o vetor dado por

$$
-\left(1-T \kappa_{2}\right) T_{1} e_{1}-\left(1-T \kappa_{1}\right) T_{2} e_{2}+\left(1-T \kappa_{1}\right)\left(1-T \kappa_{2}\right) e_{3}
$$

é normal a $X_{0}$ e, indicando por $\rho$ a sua norma, temos então

$$
\rho e_{3}^{0}=-\left(1-T \kappa_{2}\right) T_{1} e_{1}-\left(1-T \kappa_{1}\right) T_{2} e_{2}+\left(1-T \kappa_{1}\right)\left(1-T \kappa_{2}\right) e_{3} .
$$

Portanto, de (5.2), obtemos

$$
\operatorname{sen} \theta=\frac{\left(1-T \kappa_{1}\right)\left(1-T \kappa_{2}\right)}{\rho}
$$

onde $\left(1-T \kappa_{1}\right) \neq 0$ e $\left(1-T \kappa_{2}\right) \neq 0$, pois $\theta \in\left(0, \frac{\pi}{2}\right)$. Como $e_{3}^{0}$ é um campo de vetores unitário,

$$
\frac{T_{1}^{2}}{\left(1-T \kappa_{1}\right)^{2}}+\frac{T_{2}^{2}}{\left(1-T \kappa_{2}\right)^{2}}+1=\frac{1}{\operatorname{sen}^{2} \theta}
$$

Usando a notação

$$
F_{i}=\frac{T_{i}}{1-T \kappa_{i}}, \quad i=1,2
$$

podemos escrever

$$
F_{1}^{2}+F_{2}^{2}+1=\frac{1}{\operatorname{sen}^{2} \theta}
$$


e (5.5) se reduz a

$$
d X_{0}=\frac{T_{1}}{F_{1}} \omega_{1} e_{1}+\frac{T_{2}}{F_{2}} \omega_{2} e_{2}+\left(T_{1} \omega_{1}+T_{2} \omega_{2}\right) e_{3} .
$$

Considere, associado a $X_{0}$, o seguinte triedro móvel:

$$
\begin{aligned}
e_{1}^{0} & =\frac{\operatorname{sen}^{2} \theta}{\cos \theta}\left(F_{1} e_{1}+F_{2} e_{2}\right)+\cos \theta e_{3}, \\
e_{2}^{0} & =\frac{\operatorname{sen} \theta}{\cos \theta}\left(F_{2} e_{1}-F_{1} e_{2}\right), \\
e_{3}^{0} & =\operatorname{sen} \theta\left(-F_{1} e_{1}-F_{2} e_{2}+e_{3}\right) .
\end{aligned}
$$

Assim, de $d X_{0}=\omega_{1}^{0} e_{1}^{0}+\omega_{2}^{0} e_{2}^{0}$, obtemos:

$$
d X_{0}=\left(\frac{\operatorname{sen}^{2} \theta}{\cos \theta} F_{1} \omega_{1}^{0}+\frac{\operatorname{sen} \theta}{\cos \theta} F_{2} \omega_{2}^{0}\right) e_{1}+\left(\frac{\operatorname{sen}^{2} \theta}{\cos \theta} F_{2} \omega_{1}^{0}-\frac{\operatorname{sen} \theta}{\cos \theta} F_{1} \omega_{2}^{0}\right) e_{2}+\cos \theta \omega_{1}^{0} e_{3}
$$

de onde, comparando com (5.8), resulta que

$$
\begin{aligned}
\omega_{1}^{0} & =\frac{1}{\cos \theta}\left(T_{1} \omega_{1}+T_{2} \omega_{2}\right), \\
\omega_{2}^{0} & =\frac{\operatorname{sen} \theta}{\cos \theta}\left(\frac{F_{2}}{F_{1}} T_{1} \omega_{1}-\frac{F_{1}}{F_{2}} T_{2} \omega_{2}\right) .
\end{aligned}
$$

Além disso, como

$$
\begin{aligned}
d e_{3}^{0}= & \left(-F_{1} \cos \theta d \theta-\operatorname{sen} \theta d F_{1}+\operatorname{sen} \theta F_{2} \omega_{12}-\operatorname{sen} \theta \omega_{13}\right) e_{1} \\
& +\left(-F_{2} \cos \theta d \theta-\operatorname{sen} \theta F_{1} \omega_{12}-\operatorname{sen} \theta d F_{2}-\operatorname{sen} \theta \omega_{23}\right) e_{2} \\
& +\left(\cos \theta d \theta-\operatorname{sen} \theta F_{1} \omega_{13}-\operatorname{sen} \theta F_{2} \omega_{23}\right) e_{3}
\end{aligned}
$$

segue de $\omega_{i 3}^{0}=-\left\langle d e_{3}^{0}, e_{i}^{0}\right\rangle, i=1,2$, que

$$
\begin{aligned}
& \omega_{13}^{0}=-d \theta+\tan \theta\left(F_{1} \omega_{13}+F_{2} \omega_{23}\right) \\
& \omega_{23}^{0}=\frac{\operatorname{sen}^{2} \theta}{\cos \theta}\left(F_{2} d F_{1}-F_{1} d F_{2}\right)-\cos \theta \omega_{12}+\frac{\operatorname{sen}^{2} \theta}{\cos \theta}\left(F_{2} \omega_{13}-F_{1} \omega_{23}\right) .
\end{aligned}
$$

Considere os campos $V_{1}, V_{2}: U \rightarrow \mathbb{R}^{2}$ tais que

$$
e_{i}(q)=d X_{q}\left(V_{i}(q)\right), \quad i=1,2 .
$$

De (1.1), temos que

$$
\left(\omega_{i}\right)_{q}\left(V_{j}(q)\right)=\delta_{j i}, \quad i, j=1,2 .
$$

Então, sendo $\mathrm{II}^{0}=\omega_{1}^{0} \omega_{13}^{0}+\omega_{2}^{0} \omega_{23}^{0}$ a segunda forma quadrática de $X_{0}$, segue da condição imposta inicialmente (de que as direções principais de $X$ correspondem às direções conjugadas de $X_{0}$ ) que

$$
\mathrm{II}^{0}\left(V_{1}, V_{2}\right)=\mathrm{II}^{0}\left(V_{2}, V_{1}\right)=0 .
$$


Logo,

$$
\begin{aligned}
0=\mathrm{II}^{0}\left(V_{1}, V_{2}\right)= & \omega_{1}^{0}\left(V_{1}\right) \omega_{13}^{0}\left(V_{2}\right)+\omega_{2}^{0}\left(V_{1}\right) \omega_{23}^{0}\left(V_{2}\right) \\
= & \frac{1}{\cos \theta}\left[T_{1} \omega_{1}\left(V_{1}\right)+T_{2} \omega_{2}\left(V_{1}\right)\right]\left[-d \theta\left(V_{2}\right)+\tan \theta\left(F_{1} \omega_{13}\left(V_{2}\right)+F_{2} \omega_{23}\left(V_{2}\right)\right)\right] \\
& +\frac{\operatorname{sen} \theta}{\cos \theta}\left[\frac{T_{1} F_{2}}{F_{1}} \omega_{1}\left(V_{1}\right)-\frac{T_{2} F_{1}}{F_{2}} \omega_{2}\left(V_{1}\right)\right]\left[\frac{\operatorname{sen}^{2} \theta}{\cos \theta}\left(F_{2} d F_{1}\left(V_{2}\right)-F_{1} d F_{2}\left(V_{2}\right)\right)\right. \\
& \left.-\cos \theta \omega_{12}\left(V_{2}\right)+\frac{\operatorname{sen}^{2} \theta}{\cos \theta}\left(F_{2} \omega_{13}\left(V_{2}\right)-F_{1} \omega_{23}\left(V_{2}\right)\right)\right] \\
= & \frac{1}{\cos \theta}\left[-T_{1} d \theta\left(V_{2}\right)+\tan \theta T_{1}\left(F_{1} \omega_{13}\left(V_{2}\right)+F_{2} \omega_{23}\left(V_{2}\right)\right)\right. \\
& +\frac{T_{1} F_{2}}{F_{1}} \operatorname{sen} \theta\left(\frac{\operatorname{sen}^{2} \theta}{\cos \theta}\left(F_{2} d F_{1}\left(V_{2}\right)-F_{1} d F_{2}\left(V_{2}\right)\right)\right. \\
& \left.\left.-\cos \theta \omega_{12}\left(V_{2}\right)+\frac{\operatorname{sen}^{2} \theta}{\cos \theta}\left(F_{2} \omega_{13}\left(V_{2}\right)-F_{1} \omega_{23}\left(V_{2}\right)\right)\right)\right]
\end{aligned}
$$

e

$$
\begin{aligned}
0=\mathrm{II}^{0}\left(V_{2}, V_{1}\right)= & \omega_{1}^{0}\left(V_{2}\right) \omega_{13}^{0}\left(V_{1}\right)+\omega_{2}^{0}\left(V_{2}\right) \omega_{23}^{0}\left(V_{1}\right) \\
= & \frac{1}{\cos \theta}\left[-T_{2} d \theta\left(V_{1}\right)+\tan \theta T_{2}\left(F_{1} \omega_{13}\left(V_{1}\right)+F_{2} \omega_{23}\left(V_{1}\right)\right)\right. \\
& -\frac{T_{2} F_{1}}{F_{2}} \operatorname{sen} \theta\left(\frac{\operatorname{sen}^{2} \theta}{\cos \theta}\left(F_{2} d F_{1}\left(V_{1}\right)-F_{1} d F_{2}\left(V_{1}\right)\right)\right. \\
& \left.\left.-\cos \theta \omega_{12}\left(V_{1}\right)+\frac{\operatorname{sen}^{2} \theta}{\cos \theta}\left(F_{2} \omega_{13}\left(V_{1}\right)-F_{1} \omega_{23}\left(V_{1}\right)\right)\right)\right],
\end{aligned}
$$

de onde, usando (5.7), obtemos

$$
\begin{aligned}
& F_{2} d F_{1}-F_{1} d F_{2}=\frac{\cos ^{2} \theta}{\operatorname{sen}^{2} \theta}\left(\omega_{12}+F_{2} \omega_{13}-F_{1} \omega_{23}\right)+\frac{\cos \theta}{\operatorname{sen}^{3} \theta} \frac{F_{1}}{F_{2}} d \theta-\frac{\cos ^{2} \theta}{\operatorname{sen}^{4} \theta} \frac{1}{F_{2}} \omega_{13}, \\
& F_{2} d F_{1}-F_{1} d F_{2}=\frac{\cos ^{2} \theta}{\operatorname{sen}^{2} \theta}\left(\omega_{12}+F_{2} \omega_{13}-F_{1} \omega_{23}\right)-\frac{\cos \theta}{\operatorname{sen}^{3} \theta} \frac{F_{2}}{F_{1}} d \theta+\frac{\cos ^{2} \theta}{\operatorname{sen}^{4} \theta} \frac{1}{F_{1}} \omega_{23} .
\end{aligned}
$$

Subtraindo estas equações e usando que $d \theta=\theta_{1} \omega_{1}+\theta_{2} \omega_{2}$ e $\omega_{i 3}=\kappa_{i} \omega_{i}, i=1,2$, temos:

$$
\begin{aligned}
0 & =\left(\frac{F_{1}}{F_{2}}+\frac{F_{2}}{F_{1}}\right) d \theta-\frac{\cos \theta}{\operatorname{sen} \theta}\left(\frac{1}{F_{2}} \omega_{13}+\frac{1}{F_{1}} \omega_{23}\right) \\
& =\left[\left(\frac{F_{1}}{F_{2}}+\frac{F_{2}}{F_{1}}\right) \theta_{1}-\frac{\cos \theta}{\operatorname{sen} \theta} \frac{\kappa_{1}}{F_{2}}\right] \omega_{1}+\left[\left(\frac{F_{1}}{F_{2}}+\frac{F_{2}}{F_{1}}\right) \theta_{2}-\frac{\cos \theta}{\operatorname{sen} \theta} \frac{\kappa_{2}}{F_{1}}\right] \omega_{2},
\end{aligned}
$$

de onde resulta que

$$
\kappa_{i}=\frac{\cos \theta}{\operatorname{sen} \theta} \frac{1}{F_{i}} \theta_{i}, \quad i=1,2 .
$$

Agora, somando as equações (5.12), obtemos:

$$
\begin{aligned}
2\left(F_{2} d F_{1}-F_{1} d F_{2}\right)= & 2 \frac{\cos ^{2} \theta}{\operatorname{sen}^{2} \theta}\left(\omega_{12}+F_{2} \omega_{13}-F_{1} \omega_{23}\right)+\frac{\cos \theta}{\operatorname{sen}^{3} \theta}\left(\frac{F_{1}}{F_{2}}-\frac{F_{2}}{F_{1}}\right) d \theta \\
& +\frac{\cos ^{2} \theta}{\operatorname{sen}^{4} \theta}\left(-\frac{1}{F_{2}} \omega_{13}+\frac{1}{F_{1}} \omega_{23}\right)
\end{aligned}
$$

de onde segue

$$
F_{2} d F_{1}-F_{1} d F_{2}=\frac{\cos ^{2} \theta}{\operatorname{sen}^{2} \theta}\left(\omega_{12}+F_{2} \omega_{13}-F_{1} \omega_{23}\right)+\frac{\cos \theta}{\operatorname{sen}^{3} \theta}\left(-\frac{F_{2}}{F_{1}} \theta_{1} \omega_{1}+\frac{F_{1}}{F_{2}} \theta_{2} \omega_{2}\right) .
$$


Por outro lado, de (5.7), temos:

$$
F_{1} d F_{1}+F_{2} d F_{2}=-\frac{\cos \theta}{\operatorname{sen}^{3} \theta} d \theta
$$

Assim, segue de (5.13) e (5.14) que

$$
\begin{aligned}
& d F_{1}=F_{2}\left(\omega_{12}+F_{2} \omega_{13}-F_{1} \omega_{23}\right)-\frac{\cos \theta}{F_{1} \operatorname{sen}^{3} \theta} \theta_{1} \omega_{1}, \\
& d F_{2}=-F_{1}\left(\omega_{12}+F_{2} \omega_{13}-F_{1} \omega_{23}\right)-\frac{\cos \theta}{F_{2} \operatorname{sen}^{3} \theta} \theta_{2} \omega_{2} .
\end{aligned}
$$

O sistema diferencial formado por (5.7) e (5.15) deve ser satisfeito por $T$, sendo $F_{1}, F_{2}$ dadas por (5.6) e $T, \theta$ relacionadas por (5.3), se $X$ for uma superfície mínima, ou por (5.4), se $X$ for de curvatura gaussiana constante $K \neq 0$.

Supondo que $T$ é solução deste sistema, temos o seguinte

Lema 5.1.1. A 1-forma diferencial

é fechada.

$$
\psi=\frac{F_{1}}{T} \omega_{1}+\frac{F_{2}}{T} \omega_{2}
$$

Demonstração. Para provar que $d \psi=0$, isto é, que a 1-forma diferencial $\psi$ é fechada, vamos considerar $d F_{1}$ e $d F_{2}$ dadas por (5.15). Assim, segue de $d T=T_{1} \omega_{1}+T_{2} \omega_{2}$ e das equações de estrutura que

$$
\begin{aligned}
d \psi= & d\left(\frac{F_{1}}{T}\right) \wedge \omega_{1}+\frac{F_{1}}{T} d \omega_{1}+d\left(\frac{F_{2}}{T}\right) \wedge \omega_{2}+\frac{F_{2}}{T} d \omega_{2} \\
= & \frac{F_{2}}{T}\left(\omega_{12}+F_{2} \omega_{13}-F_{1} \omega_{23}\right) \wedge \omega_{1}-\frac{F_{1} T_{2}}{T^{2}} \omega_{2} \wedge \omega_{1}+\frac{F_{1}}{T} \omega_{2} \wedge \omega_{21} \\
& -\frac{F_{1}}{T}\left(\omega_{12}+F_{2} \omega_{13}-F_{1} \omega_{23}\right) \wedge \omega_{2}-\frac{F_{2} T_{1}}{T^{2}} \omega_{1} \wedge \omega_{2}+\frac{F_{2}}{T} \omega_{1} \wedge \omega_{12} \\
= & {\left[\frac{F_{1}}{T}\left(F_{2} \kappa_{2}+\frac{T_{2}}{T}\right)-\frac{F_{2}}{T}\left(F_{1} \kappa_{1}+\frac{T_{1}}{T}\right)\right] \omega_{1} \wedge \omega_{2}, }
\end{aligned}
$$

onde na última igualdade usamos que $\omega_{13}=\kappa_{1} \omega_{1}$ e $\omega_{23}=\kappa_{2} \omega_{2}$. Note que, de (5.6), resulta:

$$
\frac{F_{1}}{T}\left(F_{2} \kappa_{2}+\frac{T_{2}}{T}\right)-\frac{F_{2}}{T}\left(F_{1} \kappa_{1}+\frac{T_{1}}{T}\right)=\frac{F_{1} F_{2}}{T}\left(\kappa_{2}+\frac{T_{2}}{T F_{2}}-\kappa_{1}-\frac{T_{1}}{T F_{1}}\right)=0,
$$

pois $\kappa_{i}+\frac{T_{i}}{T F_{i}}=\frac{1}{T}, i=1,2$. Assim, concluímos a demonstração.

Como $\psi$ é uma 1-forma fechada, segue do Lema de Poincaré que existe uma função positiva $\Omega$, localmente definida, tal que

ou seja,

$$
d(\log \Omega)=\frac{F_{1}}{T} \omega_{1}+\frac{F_{2}}{T} \omega_{2}
$$

Portanto, pondo

$$
d \Omega=\frac{\Omega F_{1}}{T} \omega_{1}+\frac{\Omega F_{2}}{T} \omega_{2}
$$

resulta que

$$
W=\frac{\Omega}{T}
$$

$$
d \Omega=W F_{1} \omega_{1}+W F_{2} \omega_{2} .
$$

Às funções $\Omega$ e $W$, definidas por (5.17) e (5.16), nos referiremos como funções associadas a $T$. 


\subsection{Transformações de superfícies mínimas}

Vimos que, quando $X$ é uma superfície mínima, as funções $T$ e $\theta$ estão relacionadas por

$$
T=\frac{1}{2 c \operatorname{sen}^{2} \theta} .
$$

Assim, a equação (5.7) se reduz a

$$
F_{1}^{2}+F_{2}^{2}+1=2 c T
$$

Também, como

$$
d T=-\frac{\cos \theta}{c \operatorname{sen}^{3} \theta} d \theta
$$

usando que $d T=T_{1} \omega_{1}+T_{2} \omega_{2}$ e $d \theta=\theta_{1} \omega_{1}+\theta_{2} \omega_{2}$, resulta que

$$
c T_{i}=-\frac{\cos \theta}{\operatorname{sen}^{3} \theta} \theta_{i}, \quad i=1,2 .
$$

Portanto, de (5.6) e (5.15), obtemos

$$
\begin{aligned}
d F_{1} & =F_{2}\left(\omega_{12}+F_{2} \omega_{13}-F_{1} \omega_{23}\right)+\frac{c T_{1}}{F_{1}} \omega_{1} \\
& =F_{2}\left(\omega_{12}+F_{2} \omega_{13}-F_{1} \omega_{23}\right)+c \omega_{1}-c T \omega_{13}
\end{aligned}
$$

e

$$
\begin{aligned}
d F_{2} & =-F_{1}\left(\omega_{12}+F_{2} \omega_{13}-F_{1} \omega_{23}\right)+\frac{c T_{2}}{F_{2}} \omega_{2} \\
& =-F_{1}\left(\omega_{12}+F_{2} \omega_{13}-F_{1} \omega_{23}\right)+c \omega_{2}-c T \omega_{23} .
\end{aligned}
$$

Logo, a função $T$ deve satisfazer o sistema:

$$
\left\{\begin{array}{l}
F_{1}^{2}+F_{2}^{2}+1=2 c T, \\
d F_{1}=F_{2}\left(\omega_{12}+F_{2} \omega_{13}-F_{1} \omega_{23}\right)+c \omega_{1}-c T \omega_{13}, \\
d F_{2}=-F_{1}\left(\omega_{12}+F_{2} \omega_{13}-F_{1} \omega_{23}\right)+c \omega_{2}-c T \omega_{23},
\end{array}\right.
$$

onde $c$ é uma constante arbitrária não nula.

Para mostrar que tal $T$ existe, precisamos provar que o sistema (5.18) é integrável. Assim, vamos introduzir funções diferenciáveis auxiliares a fim de determinar um novo sistema equivalente a (5.18).

Proposição 5.2.1. Seja $X$ uma superfície mínima e considere $\left\{e_{1}, e_{2}, e_{3}\right\}$ um triedro móvel principal associado a X. Se uma função $T$ é solução do sistema (5.18), o par de funções $\Omega e$ $W$ (associadas a $T$ ) é solução do sistema:

$$
\left\{\begin{array}{l}
d \Omega_{1}=\Omega_{2} \omega_{12}+c W \omega_{1}+(c \Omega-W) \omega_{13} \\
d \Omega_{2}=-\Omega_{1} \omega_{12}+c W \omega_{2}+(c \Omega-W) \omega_{23} \\
d W=\Omega_{1} \omega_{13}+\Omega_{2} \omega_{23} \\
d \Omega=\Omega_{1} \omega_{1}+\Omega_{2} \omega_{2} \\
\Omega_{1}^{2}+\Omega_{2}^{2}+W^{2}-2 c W \Omega=0 .
\end{array}\right.
$$

Reciprocamente, se um par de funções não nulas $\Omega$ eW for solução deste sistema, então $T=\frac{\Omega}{W}$ é solução de (5.18). 
Demonstração. Suponha, inicialmente, que $T$ seja solução de (5.18) e considere as funções $\Omega$ e $W$ definidas por (5.17) e (5.16), respectivamente. Definindo as funções

$$
\Omega_{1}=W F_{1}, \quad \Omega_{2}=W F_{2},
$$

obtemos a quarta equação de (5.19):

$$
d \Omega=\Omega_{1} \omega_{1}+\Omega_{2} \omega_{2}
$$

Da derivada exterior de (5.16), temos

$$
\begin{aligned}
d W & =\frac{1}{T} d \Omega-\frac{\Omega}{T^{2}} d T \\
& =\frac{W}{T}\left(F_{1}-T_{1}\right) \omega_{1}+\frac{W}{T}\left(F_{2}-T_{2}\right) \omega_{2} \\
& =W F_{1} \kappa_{1} \omega_{1}+W F_{2} \kappa_{2} \omega_{2},
\end{aligned}
$$

onde nesta última igualdade usamos (5.6). Portanto, resulta de (5.20) que

$$
d W=\Omega_{1} \omega_{13}+\Omega_{2} \omega_{23},
$$

ou seja, a terceira equação de (5.19) está satisfeita.

Obteremos as duas primeiras equações de (5.19) a partir da derivada exterior de (5.20). De fato, usando (5.18) e (5.21), temos

$$
\begin{aligned}
d \Omega_{1} & =F_{1} d W+W d F_{1} \\
& =F_{1}\left(\Omega_{1} \omega_{13}+\Omega_{2} \omega_{23}\right)+W\left[F_{2}\left(\omega_{12}+F_{2} \omega_{13}-F_{1} \omega_{23}\right)+c \omega_{1}-c T \omega_{13}\right] \\
& =W F_{2} \omega_{12}+c W \omega_{1}+\left(W F_{2}^{2}-c W T+F_{1} \Omega_{1}\right) \omega_{13}+F_{1}\left(\Omega_{2}-W F_{2}\right) \omega_{23} \\
& =\Omega_{2} \omega_{12}+c W \omega_{1}+(c \Omega-W) \omega_{13},
\end{aligned}
$$

onde na última igualdade usamos novamente (5.18) e (5.20). Da mesma maneira, temos que

$$
\begin{aligned}
d \Omega_{2} & =F_{2} d W+W d F_{2} \\
& =F_{2}\left(\Omega_{1} \omega_{13}+\Omega_{2} \omega_{23}\right)+W\left[-F_{1}\left(\omega_{12}+F_{2} \omega_{13}-F_{1} \omega_{23}\right)+c \omega_{2}-c T \omega_{23}\right] \\
& =-W F_{1} \omega_{12}+c W \omega_{2}+F_{2}\left(\Omega_{1}-F_{1} W\right) \omega_{13}+\left(F_{2} \Omega_{2}+F_{1}^{2} W-c W T\right) \omega_{23} \\
& =-\Omega_{1} \omega_{12}+c W \omega_{2}+(c \Omega-W) \omega_{23} .
\end{aligned}
$$

Conseqüentemente, de (5.16), (5.18) e (5.20), resulta

$$
\Omega_{1}^{2}+\Omega_{2}^{2}+W^{2}-2 c \Omega W=W^{2}\left(F_{1}^{2}+F_{2}^{2}+1-2 c T\right)=0
$$

e, portanto, o par $\Omega$ e $W$ é solução do sistema (5.19).

Reciprocamente, supondo que o par $\Omega, W$ é solução não nula de (5.19), provaremos que $T=\frac{\Omega}{W}$ é solução de (5.18). Para isso, vamos primeiro definir as funções a valores reais $T_{i}, F_{i}$, $i=1,2$, do sistema (5.18). Note que

$$
\begin{aligned}
d T & =\frac{1}{W} d \Omega-\frac{\Omega}{W^{2}} d W \\
& =\frac{\Omega_{1}}{W}\left(1-\frac{\Omega}{W} \kappa_{1}\right) \omega_{1}+\frac{\Omega_{2}}{W}\left(1-\frac{\Omega}{W} \kappa_{2}\right) \omega_{2},
\end{aligned}
$$


onde na segunda igualdade usamos as expressões de (5.19) e $\omega_{i 3}=\kappa_{i} \omega_{i}, i=1,2$. Logo, defina

$$
T_{i}=\frac{\Omega_{i}}{W}\left(1-\frac{\Omega}{W} \kappa_{i}\right), \quad i=1,2
$$

e, de (5.20), considere

$$
F_{1}=\frac{\Omega_{1}}{W}, \quad F_{2}=\frac{\Omega_{2}}{W}
$$

De (5.19), temos

$$
0=\Omega_{1}^{2}+\Omega_{2}^{2}+W^{2}-2 c \Omega W=W^{2}\left(F_{1}^{2}+F_{2}^{2}+1-2 c T\right)
$$

e, como $W \neq 0$, a primeira equação de (5.18) está satisfeita. Usando novamente a (5.19) resulta

$$
\begin{aligned}
d F_{1} & =\frac{1}{W} d \Omega_{1}-\frac{\Omega_{1}}{W^{2}} d W \\
& =\frac{1}{W}\left[\Omega_{2} \omega_{12}+c W \omega_{1}+(c \Omega-W) \omega_{13}\right]-\frac{\Omega_{1}}{W^{2}}\left[\Omega_{1} \omega_{13}+\Omega_{2} \omega_{23}\right] \\
& =F_{2}\left(\omega_{12}+F_{2} \omega_{13}-F_{1} \omega_{23}\right)+c \omega_{1}-c T \omega_{13} .
\end{aligned}
$$

Analogamente,

$$
d F_{2}=-F_{1}\left(\omega_{12}+F_{2} \omega_{13}-F_{1} \omega_{23}\right)+c \omega_{2}-c T \omega_{23}
$$

o que termina a prova.

Usando o Teorema de Frobenius, mostraremos agora que o sistema diferencial (5.19) admite solução não nula.

Lema 5.2.2. Seja $X: U \subset \mathbb{R}^{2} \rightarrow \mathbb{R}^{3}$ uma superfície mínima e $\left\{e_{1}, e_{2}, e_{3}\right\}$ um triedro móvel principal associado a $X$. Então, o sistema (5.19) é integrável.

Demonstração. Considere as 1-formas diferenciais

$$
\begin{aligned}
\alpha & =d \Omega-\Omega_{1} \omega_{1}-\Omega_{2} \omega_{2}, \\
\beta_{1} & =d \Omega_{1}-\Omega_{2} \omega_{12}-c W \omega_{1}-(c \Omega-W) \omega_{13}, \\
\beta_{2} & =d \Omega_{2}+\Omega_{1} \omega_{12}-c W \omega_{2}-(c \Omega-W) \omega_{23}, \\
\delta & =d W-\Omega_{1} \omega_{13}-\Omega_{2} \omega_{23}
\end{aligned}
$$

e seja $J$ o ideal gerado por estas formas. Vamos mostrar que $J$ é fechado e, assim, do Teorema de Frobenius, segue que o sistema diferencial (5.19) é integrável (veja Apêndice B, página 146).

Das equações de estrutura e de (5.23), resulta

$$
\begin{aligned}
d \alpha= & -d \Omega_{1} \wedge \omega_{1}-\Omega_{1} d \omega_{1}-d \Omega_{2} \wedge \omega_{2}-\Omega_{2} d \omega_{2} \\
= & -\left[\beta_{1}+\Omega_{2} \omega_{12}+c W \omega_{1}+(c \Omega-W) \omega_{13}\right] \wedge \omega_{1}-\Omega_{1} \omega_{2} \wedge \omega_{21} \\
& -\left[\beta_{2}-\Omega_{1} \omega_{12}+c W \omega_{2}+(c \Omega-W) \omega_{23}\right] \wedge \omega_{2}-\Omega_{2} \omega_{1} \wedge \omega_{12} \\
= & -\beta_{1} \wedge \omega_{1}-\beta_{2} \wedge \omega_{2}+(c \Omega-W)\left(\omega_{1} \wedge \omega_{13}+\omega_{2} \wedge \omega_{23}\right) \\
= & -\beta_{1} \wedge \omega_{1}-\beta_{2} \wedge \omega_{2} .
\end{aligned}
$$

Da mesma forma, obtemos

$$
d \delta=-\beta_{1} \wedge \omega_{13}-\beta_{2} \wedge \omega_{23}
$$


Note também que

$$
\begin{aligned}
d \beta_{1}= & -d \Omega_{2} \wedge \omega_{12}-\Omega_{2} d \omega_{12}-c d W \wedge \omega_{1}-c W d \omega_{1} \\
& -(c d \Omega-d W) \wedge \omega_{13}-(c \Omega-W) d \omega_{13} \\
= & -\beta_{2} \wedge \omega_{12}-\delta \wedge\left(c \omega_{1}-\omega_{13}\right)-c \alpha \wedge \omega_{13}+c \Omega_{2}\left(\omega_{1} \wedge \omega_{23}+\omega_{13} \wedge \omega_{2}\right)
\end{aligned}
$$

e

$$
d \beta_{2}=\beta_{1} \wedge \omega_{12}-\delta \wedge\left(c \omega_{2}-\omega_{23}\right)-c \alpha \wedge \omega_{23}-c \Omega_{1}\left(\omega_{1} \wedge \omega_{23}+\omega_{13} \wedge \omega_{2}\right) .
$$

Como $X$ é uma superfície mínima, $\omega_{1} \wedge \omega_{23}+\omega_{13} \wedge \omega_{2}=0$ e, assim, podemos escrever

$$
\begin{aligned}
& d \beta_{1}=-\beta_{2} \wedge \omega_{12}-\delta \wedge\left(c \omega_{1}-\omega_{13}\right)-c \alpha \wedge \omega_{13} \\
& d \beta_{2}=\beta_{1} \wedge \omega_{12}-\delta \wedge\left(c \omega_{2}-\omega_{23}\right)-c \alpha \wedge \omega_{23} .
\end{aligned}
$$

Portanto, o ideal $J$ é fechado e, assim, do Teorema de Frobenius, existem funções diferenciáveis $\Omega, \Omega_{1}, \Omega_{2}, W$, definidas em uma vizinhança de um ponto $q \in U$, que satisfazem as quatro primeiras equações de (5.19). Além disso, decorre destas equações que

$$
\begin{aligned}
d\left(\Omega_{1}^{2}+\Omega_{2}^{2}+W^{2}-2 c W \Omega\right)= & 2 \Omega_{1} d \Omega_{1}+2 \Omega_{2} d \Omega_{2}+2(W-c \Omega) d W-2 c W d \Omega \\
= & 2 \Omega_{1}\left[\Omega_{2} \omega_{12}+c W \omega_{1}+(c \Omega-W) \omega_{13}\right] \\
& +2 \Omega_{2}\left[-\Omega_{1} \omega_{12}+c W \omega_{2}+(c \Omega-W) \omega_{23}\right] \\
& +2(W-c \Omega)\left(\Omega_{1} \omega_{13}+\Omega_{2} \omega_{23}\right)-2 c W\left(\Omega_{1} \omega_{2}+\Omega_{2} \omega_{2}\right) \\
= & 0,
\end{aligned}
$$

logo, fixando condições iniciais $\Omega(q), \Omega_{1}(q), \Omega_{2}(q), W(q)$ tal que

$$
\left(\Omega_{1}^{2}+\Omega_{2}^{2}+W^{2}-2 c W \Omega\right)(q)=0
$$

a última equação de (5.19) está satisfeita.

Teorema 5.2.3. Toda superfície mínima pode ser localmente obtida por uma família a três parâmetros de congruências de Guichard.

Demonstração. Sejam $X: U \subset \mathbb{R}^{2} \rightarrow \mathbb{R}^{3}$ uma superfície mínima e $\left\{e_{1}, e_{2}, e_{3}\right\}$ um triedro móvel associado a $X$ tal que $e_{1}, e_{2}$ são campos de direções principais. Segue do Lema 5.2.2 que, fixadas condições iniciais $\Omega(q), \Omega_{1}(q), \Omega_{2}(q), W(q)$ satisfazendo a última equação de (5.19), existem funções diferenciáveis não nulas, definidas em uma vizinhança de $q \in U$, que formam uma solução para o sistema (5.19). Considerando

$$
X_{0}=X+\frac{\Omega}{W} e_{3}
$$

provaremos que $X_{0}$ é isométrica a um parabolóide de revolução e que $X=X_{0}-\frac{\Omega}{W} e_{3}$ determina localmente uma família a três parâmetros de congruências de Guichard ${ }^{1}$.

\footnotetext{
${ }^{1}$ De fato, a solução $\frac{\Omega}{W}=T$ depende de três parâmetros que são a constante $c$ e as condições iniciais $T(q), F_{1}(q), F_{2}(q)$, ligadas pela relação (equivalente a (5.24)) dada por:
}

$$
\left(F_{1}^{2}+F_{2}^{2}+1-2 c T\right)(q)=0 .
$$


De

$$
d X_{0}=\left(\omega_{1}-\frac{\Omega}{W} \omega_{13}\right) e_{1}+\left(\omega_{2}-\frac{\Omega}{W} \omega_{23}\right) e_{2}+d\left(\frac{\Omega}{W}\right) e_{3}
$$

usando a terceira e a quarta equações de (5.19), obtemos

$$
d X_{0}=\left(\omega_{1}-\frac{\Omega}{W} \omega_{13}\right)\left(e_{1}+\frac{\Omega_{1}}{W} e_{3}\right)+\left(\omega_{2}-\frac{\Omega}{W} \omega_{23}\right)\left(e_{2}+\frac{\Omega_{2}}{W} e_{3}\right) .
$$

Como os campos $\left(e_{1}+\frac{\Omega_{1}}{W} e_{3}\right)$ e $\left(e_{2}+\frac{\Omega_{2}}{W} e_{3}\right)$ são linearmente independentes, decorre da Proposição 1.3.8 que $X_{0}$ é uma superfície parametrizada regular se, e somente se, as 1-formas $\left(\omega_{1}-\frac{\Omega}{W} \omega_{13}\right)$ e $\left(\omega_{2}-\frac{\Omega}{W} \omega_{23}\right)$ forem linearmente independentes, isto é,

$$
0 \neq\left(\omega_{1}-\frac{\Omega}{W} \omega_{13}\right) \wedge\left(\omega_{2}-\frac{\Omega}{W} \omega_{23}\right)=\left(1+\frac{\Omega^{2}}{W^{2}} K\right) \omega_{1} \wedge \omega_{2},
$$

onde $K$ é a curvatura gaussiana de $X$. Para isso, basta adicionar às condições iniciais que $\left(\frac{\Omega^{2}}{W^{2}} K+1\right)(q) \neq 0$.

Resulta também de (5.27) e da Proposição 1.3 .8 que os campos $\left(e_{1}+\frac{\Omega_{1}}{W} e_{3}\right)$ e $\left(e_{2}+\frac{\Omega_{2}}{W} e_{3}\right)$ são tangentes à superfície $X_{0}$. Logo,

$$
\frac{\Omega_{1}}{W} e_{1}+\frac{\Omega_{2}}{W} e_{2}+\frac{\Omega_{1}^{2}+\Omega_{2}^{2}}{W^{2}} e_{3} \quad \text { e } \quad \frac{\Omega_{2}}{W} e_{1}-\frac{\Omega_{1}}{W} e_{2}
$$

são também campos de vetores tangentes a $X_{0}$. Assim, definindo uma função $\theta \in\left(0, \frac{\pi}{2}\right)$ tal que

usando (5.19), temos

$$
\frac{\Omega}{W}=\frac{1}{2 c \operatorname{sen}^{2} \theta}, \quad c \neq 0,
$$

$$
\frac{\Omega_{1}^{2}}{W^{2}}+\frac{\Omega_{2}^{2}}{W^{2}}=\frac{1}{\operatorname{sen}^{2} \theta}-1=\frac{\cos ^{2} \theta}{\operatorname{sen}^{2} \theta} .
$$

Portanto, podemos considerar o seguinte triedro móvel associado a $X_{0}$ :

$$
\begin{aligned}
e_{1}^{0} & =-\frac{\operatorname{sen}^{2} \theta}{\cos \theta}\left(\frac{\Omega_{1}}{W} e_{1}+\frac{\Omega_{2}}{W} e_{2}+\frac{\cos ^{2} \theta}{\operatorname{sen}^{2} \theta} e_{3}\right), \\
e_{2}^{0} & =\frac{\operatorname{sen} \theta}{\cos \theta}\left(\frac{\Omega_{2}}{W} e_{1}-\frac{\Omega_{1}}{W} e_{2}\right), \\
e_{3}^{0} & =e_{1}^{0} \wedge e_{2}^{0}=-\operatorname{sen} \theta\left(\frac{\Omega_{1}}{W} e_{1}+\frac{\Omega_{2}}{W} e_{2}-e_{3}\right) .
\end{aligned}
$$

Observe agora que, como

resulta de (5.26) que

$$
d\left(\frac{\Omega}{W}\right)=-\frac{\cos \theta}{c \operatorname{sen}^{3} \theta} d \theta
$$

$$
d X_{0}=\left(\omega_{1}-\frac{\Omega}{W} \omega_{13}\right) e_{1}+\left(\omega_{2}-\frac{\Omega}{W} \omega_{23}\right) e_{2}-\frac{\cos \theta}{c \operatorname{sen}^{3} \theta} d \theta e_{3} .
$$

Por outro lado, de (5.29), temos

$$
\begin{aligned}
d X_{0}= & \omega_{1}^{0} e_{1}^{0}+\omega_{2}^{0} e_{2}^{0} \\
= & \frac{\operatorname{sen} \theta}{\cos \theta}\left(-\operatorname{sen} \theta \frac{\Omega_{1}}{W} \omega_{1}^{0}+\frac{\Omega_{2}}{W} \omega_{2}^{0}\right) e_{1} \\
& +\frac{\operatorname{sen} \theta}{\cos \theta}\left(-\operatorname{sen} \theta \frac{\Omega_{2}}{W} \omega_{1}^{0}-\frac{\Omega_{1}}{W} \omega_{2}^{0}\right) e_{2}-\cos \theta \omega_{1}^{0} e_{3},
\end{aligned}
$$


de onde, comparando com (5.30), obtemos

$$
\begin{aligned}
\omega_{1}^{0} & =\frac{1}{c \operatorname{sen}^{3} \theta} d \theta, \\
\omega_{2}^{0} & =\frac{\operatorname{sen} \theta}{\cos \theta}\left[\frac{\Omega_{2}}{W}\left(\omega_{1}-\frac{\Omega}{W} \omega_{13}\right)-\frac{\Omega_{1}}{W}\left(\omega_{2}-\frac{\Omega}{W} \omega_{23}\right)\right] .
\end{aligned}
$$

Também, de (5.29), temos

$$
\begin{aligned}
d e_{1}^{0}= & {\left[-\left(2 \operatorname{sen} \theta+\frac{\operatorname{sen}^{3} \theta}{\cos ^{2} \theta}\right) \frac{\Omega_{1}}{W} d \theta-\frac{\operatorname{sen}^{2} \theta}{\cos \theta}\left(\frac{1}{W} d \Omega_{1}-\frac{\Omega_{1}}{W^{2}} d W-\frac{\Omega_{2}}{W} \omega_{12}-\frac{\cos ^{2} \theta}{\operatorname{sen}^{2} \theta} \omega_{13}\right)\right] e_{1} } \\
& +\left[-\left(2 \operatorname{sen} \theta+\frac{\operatorname{sen}^{3} \theta}{\cos ^{2} \theta}\right) \frac{\Omega_{2}}{W} d \theta-\frac{\operatorname{sen}^{2} \theta}{\cos \theta}\left(\frac{\Omega_{1}}{W} \omega_{12}+\frac{1}{W} d \Omega_{2}-\frac{\Omega_{2}}{W^{2}} d W-\frac{\cos ^{2} \theta}{\operatorname{sen}^{2} \theta} \omega_{23}\right)\right] e_{2} \\
& +\left[-\left(2 \operatorname{sen} \theta+\frac{\operatorname{sen}^{3} \theta}{\cos \theta}\right) \frac{\cos ^{2} \theta}{\operatorname{sen}^{2} \theta} d \theta-\frac{\operatorname{sen}^{2} \theta}{\cos \theta}\left(\frac{\Omega_{1}}{W} \omega_{13}+\frac{\Omega_{2}}{W} \omega_{23}-2 \frac{\cos \theta}{\operatorname{sen}^{3} \theta} d \theta\right)\right] e_{3} .
\end{aligned}
$$

Portanto, usando (5.19),

$$
\begin{aligned}
\omega_{12}^{0} & =\left\langle d e_{1}^{0}, e_{2}^{0}\right\rangle \\
& =-\frac{\operatorname{sen}^{3} \theta}{\cos ^{2} \theta}\left[\left(\frac{\Omega_{2}}{W^{2}} d \Omega_{1}-\frac{\Omega_{1}}{W^{2}} d \Omega_{2}\right)-\left(\frac{\Omega_{1}^{2}}{W^{2}}+\frac{\Omega_{2}^{2}}{W^{2}}\right) \omega_{12}-\frac{\cos ^{2} \theta}{\operatorname{sen}^{2} \theta}\left(\frac{\Omega_{2}}{W} \omega_{13}-\frac{\Omega_{1}}{W} \omega_{23}\right)\right] \\
& =-\frac{\operatorname{sen}^{3} \theta}{\cos ^{2} \theta}\left[\frac{\Omega_{2}}{W}\left(c \omega_{1}+\left(c \frac{\Omega}{W}-1-\frac{\cos ^{2} \theta}{\operatorname{sen}^{2} \theta}\right) \omega_{13}\right)+\frac{\Omega_{1}}{W}\left(c \omega_{2}-\left(c \frac{\Omega}{W}-1-\frac{\cos ^{2} \theta}{\operatorname{sen}^{2} \theta}\right) \omega_{23}\right)\right] \\
& =-c \frac{\operatorname{sen}^{3} \theta}{\cos ^{2} \theta}\left[\frac{\Omega_{2}}{W}\left(\omega_{1}-\frac{\Omega}{W} \omega_{13}\right)-\frac{\Omega_{1}}{W}\left(\omega_{2}-\frac{\Omega}{W} \omega_{23}\right)\right],
\end{aligned}
$$

isto é,

Como, usando (5.29)

$$
\omega_{12}^{0}=-c \frac{\operatorname{sen}^{2} \theta}{\cos \theta} \omega_{2}^{0}
$$

$$
\begin{aligned}
d \omega_{12}^{0} & =-c\left(2 \operatorname{sen} \theta+\frac{\operatorname{sen}^{3} \theta}{\cos ^{2} \theta}\right) d \theta \wedge \omega_{2}^{0}-c \frac{\operatorname{sen}^{2} \theta}{\cos \theta} d \omega_{2}^{0} \\
& =-c \frac{1}{\cos \theta}\left[\left(2 \operatorname{sen} \theta \cos \theta+\frac{\operatorname{sen}^{3} \theta}{\cos \theta}\right) c \operatorname{sen}^{3} \theta+\operatorname{sen}^{2} \theta\left(-c \frac{\operatorname{sen}^{2} \theta}{\cos \theta}\right)\right] \omega_{1}^{0} \wedge \omega_{2}^{0} \\
& =-c^{2} \operatorname{sen}^{4} \theta \omega_{1}^{0} \wedge \omega_{2}^{0},
\end{aligned}
$$

resulta da equação de Gauss, $d \omega_{12}^{0}=-K_{0} \omega_{1}^{0} \wedge \omega_{2}^{0}$, que

$$
K_{0}=c^{2} \operatorname{sen}^{4} \theta \text {. }
$$

Agora, observe que de (5.29) temos

$$
\cos \theta e_{1}^{0}-\operatorname{sen} \theta e_{3}^{0}=-e_{3}
$$

e, portanto, (5.25) resulta em

$$
X=X_{0}+\frac{\Omega}{W}\left(\cos \theta e_{1}^{0}-\operatorname{sen} \theta e_{3}^{0}\right),
$$

que é uma congruência de retas normal a $X$, por construção. Além disso, usando as notações:

$$
\begin{aligned}
& T=\frac{\Omega}{W}, \\
& \omega_{12}^{0}=A \omega_{1}^{0}+B \omega_{2}^{0}, \\
& d \theta=\theta_{1}^{0} \omega_{1}^{0}+\theta_{2}^{0} \omega_{2}^{0},
\end{aligned}
$$


de $(5.28),(5.31),(5.32)$ e (5.33) resultam as relações de (4.33) a (4.36):

$$
\begin{aligned}
& B \operatorname{sen} \theta \cos \theta+\theta_{1}^{0}=0, \\
& A=\theta_{2}^{0}=0, \\
& \operatorname{sen} \theta-2 T \theta_{1}^{0}=0, \\
& K_{0} \operatorname{sen}^{2} \theta-\left(\theta_{1}^{0}\right)^{2}=0 .
\end{aligned}
$$

Portanto, da prova do Teorema 4.3.1, concluímos que $X_{0}$ é isométrica a um parabolóide de revolução e que (5.34) determina uma família a três parâmetros de congruências de Guichard.

Corolário 5.2.4. A toda superfície mínima sem pontos planares, podemos associar uma família a três parâmetros de superfícies minimas.

Demonstração. Considere $X: U \subset \mathbb{R}^{2} \rightarrow \mathbb{R}^{3}$ uma superfície mínima sem pontos planares. Decorre do Teorema 5.2.3 que existe uma família a três parâmetros de congruências de Guichard

$$
X=X_{0}-\frac{\Omega}{W} e_{3}=X_{0}+\frac{\Omega}{W}\left(\cos \theta e_{1}^{0}-\operatorname{sen} \theta e_{3}^{0}\right),
$$

sendo $X_{0}$ uma superfície isométrica a um parabolóide de revolução. Então, do Corolário 4.2.7 resulta que a superfície $X^{s}$, simétrica a $X$ em relação a $X_{0}$, dada por

$$
X^{s}=X_{0}+\frac{\Omega}{W}\left(\cos \theta e_{1}^{0}+\operatorname{sen} \theta e_{3}^{0}\right)
$$

é também uma superfície mínima. Relacionando estas duas equações e usando (5.28) e (5.29), temos

$$
\begin{aligned}
X^{s} & =X+2 \frac{\Omega}{W} \operatorname{sen} \theta e_{3}^{0} \\
& =X-\frac{1}{c}\left(\frac{\Omega_{1}}{W} e_{1}+\frac{\Omega_{2}}{W} e_{2}-e_{3}\right),
\end{aligned}
$$

que é uma família a três parâmetros de superfícies mínimas associadas a $X$.

Note que, de fato, $X^{s}$ é uma superfície. Diferenciando (5.35), temos

$$
d X^{s}=d X-\frac{1}{c}\left[\left(\frac{1}{W} d \Omega_{1}-\frac{\Omega_{1}}{W^{2}} d W\right) e_{1}+\frac{\Omega_{1}}{W} d e_{1}+\left(\frac{1}{W} d \Omega_{2}-\frac{\Omega_{2}}{W^{2}} d W\right) e_{2}+\frac{\Omega_{2}}{W} d e_{2}-d e_{3}\right]
$$

de onde, usando (5.19), obtemos

$$
\begin{aligned}
d X^{s}= & \omega_{13} \frac{1}{c W^{2}}\left[\left(\Omega_{1}^{2}-c \Omega W\right) e_{1}+\Omega_{1} \Omega_{2} e_{2}-\Omega_{1} W e_{3}\right] \\
& +\omega_{23} \frac{1}{c W^{2}}\left[\Omega_{1} \Omega_{2} e_{1}+\left(\Omega_{2}^{2}-c \Omega W\right) e_{2}-\Omega_{2} W e_{3}\right] .
\end{aligned}
$$

Como a superfície $X$ não possui pontos planares, temos que

$$
\omega_{13} \wedge \omega_{23}=K \omega_{1} \wedge \omega_{2} \neq 0
$$

Segue, assim, da Proposição 1.3.8 que $X^{s}$ é uma superfície parametrizada regular. 
Observação 5.2.5. Decorre da Proposição 1.3 .8 e de (5.36) que os campos de vetores dados por:

$$
\begin{aligned}
& \left(\Omega_{1}^{2}-c \Omega W\right) e_{1}+\Omega_{1} \Omega_{2} e_{2}-\Omega_{1} W e_{3} \\
& \Omega_{1} \Omega_{2} e_{1}+\left(\Omega_{2}^{2}-c \Omega W\right) e_{2}-\Omega_{2} W e_{3}
\end{aligned}
$$

são tangentes à superfície $X^{s}$. Assim,

$$
\begin{aligned}
e_{1}^{s} & =\frac{1}{c \Omega W}\left[\left(\Omega_{1}^{2}-c \Omega W\right) e_{1}+\Omega_{1} \Omega_{2} e_{2}-\Omega_{1} W e_{3}\right], \\
e_{2}^{s} & =\frac{1}{c \Omega W}\left[\Omega_{1} \Omega_{2} e_{1}+\left(\Omega_{2}^{2}-c \Omega W\right) e_{2}-\Omega_{2} W e_{3}\right], \\
e_{3}^{s} & =e_{1}^{s} \wedge e_{2}^{s}=\frac{1}{c \Omega}\left[-\Omega_{1} e_{1}-\Omega_{2} e_{2}+(W-c \Omega) e_{3}\right]
\end{aligned}
$$

é um triedro móvel associado a $X^{s}$. Como $d X^{s}=\omega_{1}^{s} e_{1}^{s}+\omega_{2}^{s} e_{2}^{s}$, comparando com (5.36) obtemos

$$
\begin{aligned}
& \omega_{1}^{s}=\frac{\Omega}{W} \omega_{13}, \\
& \omega_{2}^{s}=\frac{\Omega}{W} \omega_{23} .
\end{aligned}
$$

Temos também que, de (5.37),

$$
\begin{aligned}
d e_{3}^{s}= & \frac{1}{c \Omega^{2}}\left[\left(\Omega_{1}^{2}-c \Omega W\right) \omega_{1}+\Omega_{1} \Omega_{2} \omega_{2}\right] e_{1}+\frac{1}{c \Omega^{2}}\left[\Omega_{1} \Omega_{2} \omega_{1}+\left(\Omega_{2}^{2}-c \Omega W\right) \omega_{2}\right] e_{2} \\
& -\frac{1}{c \Omega^{2}}\left[W\left(\Omega_{1} \omega_{1}+\Omega_{2} \omega_{2}\right)\right] e_{3} .
\end{aligned}
$$

Assim, usando a última relação de (5.19),

$$
\begin{aligned}
\omega_{13}^{s} & =-\left\langle d e_{3}^{s}, e_{1}^{s}\right\rangle \\
& =-\frac{1}{c^{2} \Omega^{3} W}\left[\left(\Omega_{1}^{2}+\Omega_{2}^{2}+W^{2}-2 c \Omega W\right)\left(\Omega_{1}^{2} \omega_{1}+\Omega_{1} \Omega_{2} \omega_{2}\right)+c^{2} \Omega^{2} W^{2} \omega_{1}\right] \\
& =-\frac{W}{\Omega} \omega_{1}
\end{aligned}
$$

e, analogamente,

$$
\omega_{23}^{s}=-\left\langle d e_{3}^{s}, e_{2}^{s}\right\rangle=-\frac{W}{\Omega} \omega_{2}
$$

Portanto,

$$
\begin{aligned}
& \omega_{13}^{s}=-\frac{W}{\Omega} \omega_{1}, \\
& \omega_{23}^{s}=-\frac{W}{\Omega} \omega_{2} .
\end{aligned}
$$

\subsection{Transformações de superfícies de curvatura gaussiana con- stante não nula}

Se $X: U \subset \mathbb{R}^{2} \rightarrow \mathbb{R}^{3}$ é uma superfície parametrizada regular de curvatura gaussiana constante não nula, vimos que as funções $T$ e $\theta$ estão relacionadas por

$$
T^{2}=\frac{1}{K}+\frac{1}{c \operatorname{sen}^{2} \theta}, \quad c \neq 0
$$


Logo, a relação (5.7) se reduz a

$$
F_{1}^{2}+F_{2}^{2}+1=c\left(T^{2}-\frac{1}{K}\right)
$$

Como

$$
T d T=-\frac{\cos \theta}{c \operatorname{sen}^{3} \theta} d \theta
$$

usando $d T=T_{1} \omega_{1}+T_{2} \omega_{2}$ e $d \theta=\theta_{1} \omega_{1}+\theta_{2} \omega_{2}$, segue que

$$
c T T_{i}=-\frac{\cos \theta}{\operatorname{sen}^{3} \theta} \theta_{i}, \quad i=1,2 .
$$

Assim, de (5.6), as equações (5.15) se reduzem a

$$
\begin{aligned}
d F_{1} & =F_{2}\left(\omega_{12}+F_{2} \omega_{13}-F_{1} \omega_{23}\right)+\frac{c T T_{1}}{F_{1}} \omega_{1} \\
& =F_{2}\left(\omega_{12}+F_{2} \omega_{13}-F_{1} \omega_{23}\right)+c T \omega_{1}-c T^{2} \omega_{13}, \\
d F_{2} & =-F_{1}\left(\omega_{12}+F_{2} \omega_{13}-F_{1} \omega_{23}\right)+\frac{c T T_{2}}{F_{2}} \omega_{2} \\
& =-F_{1}\left(\omega_{12}+F_{2} \omega_{13}-F_{1} \omega_{23}\right)+c T \omega_{2}-c T^{2} \omega_{23} .
\end{aligned}
$$

Portanto, verificar que $T$ satisfaz o sistema dado por (5.7) e (5.15) é equivalente a verificar que $T$ satisfaz:

$$
\left\{\begin{array}{l}
F_{1}^{2}+F_{2}^{2}+1=c\left(T^{2}-\frac{1}{K}\right), \\
d F_{1}=F_{2}\left(\omega_{12}+F_{2} \omega_{13}-F_{1} \omega_{23}\right)+c T \omega_{1}-c T^{2} \omega_{13}, \\
d F_{2}=-F_{1}\left(\omega_{12}+F_{2} \omega_{13}-F_{1} \omega_{23}\right)+c T \omega_{2}-c T^{2} \omega_{23},
\end{array}\right.
$$

onde $F_{i}=\frac{T_{i}}{1-T \kappa_{i}}, i=1,2$, e $c$ é uma constante arbitrária não nula.

Para mostrar que tal $T$ existe, precisamos provar que o sistema (5.40) admite solução não nula. Assim, vamos introduzir funções diferenciáveis auxiliares, reduzindo (5.40) a um sistema linear e homogêneo, equivalente a ele, cuja integrabilidade provaremos em seguida.

Proposição 5.3.1. Sejam $X: U \subset \mathbb{R}^{2} \rightarrow \mathbb{R}^{3}$ uma superfície parametrizada regular de curvatura gaussiana constante $K \neq 0$ e $\left\{e_{1}, e_{2}, e_{3}\right\}$ um triedro móvel principal associado a $X$. Se $T$ for solução do sistema (5.40), então o par de funções $\Omega, W$ (associadas a $T$ ) é solução do sistema:

$$
\left\{\begin{array}{l}
d \Omega_{1}=\Omega_{2} \omega_{12}+c \Omega \omega_{1}-W\left(\frac{c}{K}+1\right) \omega_{13} \\
d \Omega_{2}=-\Omega_{1} \omega_{12}+c \Omega \omega_{2}-W\left(\frac{c}{K}+1\right) \omega_{23} \\
d W=\Omega_{1} \omega_{13}+\Omega_{2} \omega_{23} \\
d \Omega=\Omega_{1} \omega_{1}+\Omega_{2} \omega_{2} \\
\Omega_{1}^{2}+\Omega_{2}^{2}+W^{2}-c\left(\Omega^{2}-\frac{W^{2}}{K}\right)=0
\end{array}\right.
$$

Reciprocamente, se um par de funções não nulas $\Omega, W$ for solução deste sistema, então $T=\frac{\Omega}{W}$ é solução de (5.40). 
Demonstração. Suponha que $T$ seja solução do sistema (5.40) e considere as funções $\Omega$ e $W$ definidas em (5.17) e (5.16). Definindo $\Omega_{1}, \Omega_{2}$ por (5.20), como na prova da Proposição 5.2.1, obtemos a terceira e quarta equações de (5.41):

$$
\begin{aligned}
d W & =\Omega_{1} \omega_{13}+\Omega_{2} \omega_{23}, \\
d \Omega & =\Omega_{1} \omega_{1}+\Omega_{2} \omega_{2} .
\end{aligned}
$$

De (5.20) temos:

$$
\begin{aligned}
d \Omega_{1} & =W d F_{1}+F_{1} d W \\
& =W F_{2} \omega_{12}+c T W \omega_{1}+\left(W F_{2}^{2}-c T^{2} W+F_{1} \Omega_{1}\right) \omega_{13}+F_{1}\left(-W F_{2}+\Omega_{2}\right) \omega_{23} \\
& =\Omega_{2} \omega_{12}+c \Omega \omega_{1}-W\left(\frac{c}{K}+1\right) \omega_{13},
\end{aligned}
$$

onde usamos (5.16), (5.20) e (5.40). Analogamente,

$$
d \Omega_{2}=-\Omega_{1} \omega_{12}+c \Omega \omega_{2}-W\left(\frac{c}{K}+1\right) \omega_{23}
$$

Portanto, falta somente verificar a última equação de (5.41). Usando (5.16) e (5.20), resulta da primeira relação de $(5.40)$ que

$$
\Omega_{1}^{2}+\Omega_{2}^{2}+W^{2}-c\left(\Omega^{2}-\frac{W^{2}}{K}\right)=W^{2}\left[F_{1}^{2}+F_{2}^{2}+1-c\left(T^{2}-\frac{1}{K}\right)\right]=0 .
$$

Reciprocamente, supondo que o par de funções $\Omega, W$ é uma solução não nula de (5.41), vamos provar que $T=\frac{\Omega}{W}$ é solução de (5.40). Note que, como $e_{1}, e_{2}$ são campos de direções principais, resulta de (5.41):

$$
d T=\frac{\Omega_{1}}{W}\left(1-\frac{\Omega}{W} \kappa_{1}\right) \omega_{1}+\frac{\Omega_{2}}{W}\left(1-\frac{\Omega}{W} \kappa_{2}\right) \omega_{2} .
$$

Assim, defina

$$
T_{i}=\frac{\Omega_{i}}{W}\left(1-\frac{\Omega}{W} \kappa_{i}\right) \omega_{i}, \quad i=1,2
$$

e

$$
F_{1}=\frac{\Omega_{1}}{W}, \quad F_{2}=\frac{\Omega_{2}}{W} .
$$

Então, segue da última relação de (5.41) que

$$
\begin{aligned}
0 & =\Omega_{1}^{2}+\Omega_{2}^{2}+W^{2}-c\left(\Omega^{2}-\frac{W^{2}}{K}\right) \\
& =W^{2}\left[F_{1}^{2}+F_{2}^{2}+1-c\left(T^{2}-\frac{1}{K}\right)\right]
\end{aligned}
$$

e, como $W \neq 0$, obtemos $F_{1}^{2}+F_{2}^{2}+1=c\left(T^{2}-\frac{1}{K}\right)$.

Calculando a diferencial de $F_{1}$ e $F_{2}$, temos

$$
\begin{aligned}
d F_{1} & =\frac{1}{W} d \Omega_{1}-\frac{\Omega_{1}}{W^{2}} d W \\
& =F_{2} \omega_{2}+c T \omega_{1}-\left(\frac{c}{K}+1+F_{1}^{2}\right) \omega_{13}-F_{1} F_{2} \omega_{23} \\
& =c T \omega_{1}+F_{2}\left(\omega_{12}+F_{2} \omega_{13}-F_{1} \omega_{23}\right)-c T^{2} \omega_{13}
\end{aligned}
$$


e

$$
d F_{2}=c T \omega_{2}-F_{1}\left(\omega_{12}+F_{2} \omega_{13}-F_{1} \omega_{23}\right)-c T^{2} \omega_{23},
$$

ou seja, as duas últimas equações de (5.40).

Decorre do Teorema de Frobenius que o sistema (5.41) admite solução não nula. Mais precisamente,

Lema 5.3.2. Sejam $X: U \subset \mathbb{R}^{2} \rightarrow \mathbb{R}^{3}$ uma superfície parametrizada regular de curvatura gaussiana constante $K \neq 0$ e $\left\{e_{1}, e_{2}, e_{3}\right\}$ um triedro móvel principal associado a $X$. Então, o sistema (5.41) é integrável.

Demonstração. Considere as 1-formas diferenciais dadas por:

$$
\begin{aligned}
\alpha & =d \Omega-\Omega_{1} \omega_{1}-\Omega_{2} \omega_{2} \\
\beta_{1} & =d \Omega_{1}-\Omega_{2} \omega_{12}-c \Omega \omega_{1}-W\left(\frac{c}{K}-1\right) \omega_{13}, \\
\beta_{2} & =d \Omega_{2}+\Omega_{1} \omega_{12}-c \Omega \omega_{2}-W\left(\frac{c}{K}-1\right) \omega_{23} \\
\delta & =d W-\Omega_{1} \omega_{13}-\Omega_{2} \omega_{23} .
\end{aligned}
$$

Vamos provar que o ideal $J$ gerado por estas formas é fechado. De fato:

$$
\begin{aligned}
d \alpha & =-d \Omega_{1} \wedge \omega_{1}-\Omega_{1} d \omega_{1}-d \Omega_{2} \wedge \omega_{2}-\Omega_{2} d \omega_{2} \\
& =-\beta_{1} \wedge \omega_{1}-\beta_{2} \wedge \omega_{2}
\end{aligned}
$$

e

$$
\begin{aligned}
d \delta & =-d \Omega_{1} \wedge \omega_{13}-\Omega_{1} d \omega_{13}-d \Omega_{2} \wedge \omega_{23}-\Omega_{2} d \omega_{23} \\
& =-\beta_{1} \wedge \omega_{13}-\beta_{2} \wedge \omega_{23}
\end{aligned}
$$

Da mesma forma, obtemos

$$
\begin{aligned}
d \beta_{1} & =-d \Omega_{2} \wedge \omega_{12}-\Omega_{2} d \omega_{12}-c d \Omega \wedge \omega_{1}-c \Omega d \omega_{1}+\left(\frac{c}{K}+1\right)\left(d W \wedge \omega_{13}+W d \omega_{13}\right) \\
& =-\beta_{2} \wedge \omega_{12}-c \alpha \wedge \omega_{1}+\left(\frac{c}{K}+1\right) \delta \wedge \omega_{13}-c \Omega_{2} \omega_{2} \wedge \omega_{1}-\frac{c}{K} \Omega_{2} \omega_{13} \wedge \omega_{23}
\end{aligned}
$$

$\mathrm{e}$

$$
d \beta_{2}=\beta_{1} \wedge \omega_{12}-c \alpha \wedge \omega_{2}+\left(\frac{c}{K}+1\right) \delta \wedge \omega_{23}-c \Omega_{1} \omega_{1} \wedge \omega_{2}+\frac{c}{K} \Omega_{1} \omega_{13} \wedge \omega_{23} .
$$

Usando, agora, a equação de Gauss, $\omega_{13} \wedge \omega_{23}=K \omega_{1} \wedge \omega_{2}$, podemos escrever

$$
\begin{aligned}
& d \beta_{1}=-\beta_{2} \wedge \omega_{12}-c \alpha \wedge \omega_{1}+\left(\frac{c}{K}+1\right) \delta \wedge \omega_{13}, \\
& d \beta_{2}=\beta_{1} \wedge \omega_{12}-c \alpha \wedge \omega_{2}+\left(\frac{c}{K}+1\right) \delta \wedge \omega_{23} .
\end{aligned}
$$

Portanto, como o ideal $J$ é fechado, pelo Teorema de Frobenius, existem funções diferenciáveis $\Omega, \Omega_{1}, \Omega_{2}, W$, definidas em uma vizinhança de um ponto $q \in U$, que satisfazem as quatro 
primeiras equações de (5.41). Além disso, destas relações resulta que

$$
\begin{aligned}
d\left(\Omega_{1}^{2}\right. & \left.+\Omega_{2}^{2}+W^{2}-c \Omega^{2}+\frac{c}{K} W^{2}\right) \\
& =2 \Omega_{1} d \Omega_{1}+2 \Omega_{2} d \Omega_{2}-2 c \Omega d \Omega+2\left(1+\frac{c}{K}\right) W d W \\
& =0
\end{aligned}
$$

logo, fixando condições iniciais $\Omega(q), \Omega_{1}(q), \Omega_{2}(q), W(q)$ tais que

$$
\left(\Omega_{1}^{2}+\Omega_{2}^{2}+W^{2}-c \Omega^{2}+\frac{c}{K} W^{2}\right)(q)=0,
$$

obtemos que vale a última equação do sistema (5.41).

Obtida uma solução do sistema (5.41), temos o seguinte resultado.

Teorema 5.3.3. Toda superfície de curvatura gaussiana constante $K \neq 0$ pode ser localmente obtida por uma familia a três parâmetros de congruências de Guichard.

Demonstração. Sejam $X: U \subset \mathbb{R}^{2} \rightarrow \mathbb{R}^{3}$ uma superfície de curvatura gaussiana constante $K \neq 0$ e $\left\{e_{1}, e_{2}, e_{3}\right\}$ um triedro móvel principal associado a $X$. Decorre do Lema 5.3.2 que, fixadas condições iniciais não nulas $\Omega(q), \Omega_{1}(q), \Omega_{2}(q), W(q), q \in U$, satisfazendo a última equação de (5.41), existem funções diferenciáveis $\Omega, \Omega_{1}, \Omega_{2}, W$, definidas em uma vizinhança de $q$, que formam uma solução para o sistema (5.41).

Considerando

$$
X_{0}=X+\frac{\Omega}{W} e_{3}
$$

mostraremos que:

- se $K>0$, então $X_{0}$ é isométrica a um elipsóide de revolução ou a um hiperbolóide de revolução de duas folhas;

- se $K<0$, então $X_{0}$ é isométrica a uma das superfícies da Definição 4.3.3.

Além disso,

$$
X=X_{0}-\frac{\Omega}{W} e_{3}
$$

é uma família a três parâmetros de congruências de Guichard.

De (5.42), temos

$$
d X_{0}=\left(\omega_{1}-\frac{\Omega}{W} \omega_{13}\right) e_{1}+\left(\omega_{2}-\frac{\Omega}{W} \omega_{23}\right) e_{2}+d\left(\frac{\Omega}{W}\right) e_{3}
$$

de onde, usando as relações (5.41), obtemos

$$
d X_{0}=\left(\omega_{1}-\frac{\Omega}{W} \omega_{13}\right)\left(e_{1}+\frac{\Omega_{1}}{W} e_{3}\right)+\left(\omega_{2}-\frac{\Omega}{W} \omega_{23}\right)\left(e_{2}+\frac{\Omega_{2}}{W} e_{3}\right) .
$$

Como os campos $\left(e_{1}+\frac{\Omega_{1}}{W} e_{3}\right)$ e $\left(e_{2}+\frac{\Omega_{2}}{W} e_{3}\right)$ são linearmente independentes, da Proposição 1.3.8 resulta que $X_{0}$ é uma superfície parametrizada regular se, e somente se, as 1-formas $\left(\omega_{1}-\frac{\Omega}{W} \omega_{13}\right)$ e $\left(\omega_{2}-\frac{\Omega}{W} \omega_{23}\right)$ forem linearmente independentes, isto é,

$$
0 \neq\left(\omega_{1}-\frac{\Omega}{W} \omega_{13}\right) \wedge\left(\omega_{2}-\frac{\Omega}{W} \omega_{23}\right)=\left(1-\frac{\Omega}{W} \kappa_{1}\right)\left(1-\frac{\Omega}{W} \kappa_{2}\right) \omega_{1} \wedge \omega_{2}
$$


Assim, basta adicionarmos às condições iniciais que as curvaturas principais da superfície $X$ no ponto $q$ são diferentes de $\frac{W}{\Omega}(q)$.

Definindo a função $\theta \in\left(0, \frac{\pi}{2}\right)$ tal que

$$
\frac{\Omega^{2}}{W^{2}}=\frac{1}{K}+\frac{1}{c \operatorname{sen}^{2} \theta}
$$

de (5.41) temos que

$$
\frac{\Omega_{1}^{2}}{W^{2}}+\frac{\Omega_{2}^{2}}{W^{2}}=c\left(\frac{\Omega^{2}}{W^{2}}-\frac{1}{K}\right)-1=\frac{\cos ^{2} \theta}{\operatorname{sen}^{2} \theta}
$$

e, assim, (como feito na demonstração do Teorema 5.2.3) vamos considerar o seguinte triedro móvel associado a $X_{0}$ :

$$
\begin{aligned}
e_{1}^{0} & =-\frac{\operatorname{sen}^{2} \theta}{\cos \theta}\left(\frac{\Omega_{1}}{W} e_{1}+\frac{\Omega_{2}}{W} e_{2}+\frac{\cos ^{2} \theta}{\operatorname{sen}^{2} \theta} e_{3}\right), \\
e_{2}^{0} & =\frac{\operatorname{sen} \theta}{\cos \theta}\left(\frac{\Omega_{2}}{W} e_{1}-\frac{\Omega_{1}}{W} e_{2}\right) \\
e_{3}^{0} & =-\operatorname{sen} \theta\left(\frac{\Omega_{1}}{W} e_{1}+\frac{\Omega_{2}}{W} e_{2}-e_{3}\right) .
\end{aligned}
$$

Note que, de (5.45), temos

$$
d\left(\frac{\Omega}{W}\right)=-\frac{W}{\Omega} \frac{\cos \theta}{c \operatorname{sen}^{3} \theta} d \theta
$$

e, portanto, reescrevemos (5.43) como

$$
d X_{0}=\left(\omega_{1}-\frac{\Omega}{W} \omega_{13}\right) e_{1}+\left(\omega_{2}-\frac{\Omega}{W} \omega_{23}\right) e_{2}-\frac{W}{\Omega} \frac{\cos \theta}{c \operatorname{sen}^{3} \theta} d \theta e_{3} .
$$

Por outro lado, usando (5.46),

$$
\begin{aligned}
d X_{0}= & \omega_{1}^{0} e_{1}^{0}+\omega_{2}^{0} e_{2}^{0} \\
= & \frac{\operatorname{sen} \theta}{\cos \theta}\left(-\operatorname{sen} \theta \frac{\Omega_{1}}{W} \omega_{1}^{0}+\frac{\Omega_{2}}{W} \omega_{2}^{0}\right) e_{1} \\
& +\frac{\operatorname{sen} \theta}{\cos \theta}\left(-\operatorname{sen} \theta \frac{\Omega_{2}}{W} \omega_{1}^{0}-\frac{\Omega_{1}}{W} \omega_{2}^{0}\right) e_{2}-\cos \theta \omega_{1}^{0} e_{3},
\end{aligned}
$$

de onde, comparando com (5.47), obtemos

$$
\begin{aligned}
& \omega_{1}^{0}=\frac{W}{\Omega} \frac{1}{c \operatorname{sen}^{3} \theta} d \theta, \\
& \omega_{2}^{0}=\frac{\operatorname{sen} \theta}{\cos \theta}\left[\frac{\Omega_{2}}{W}\left(\omega_{1}-\frac{\Omega}{W} \omega_{13}\right)-\frac{\Omega_{1}}{W}\left(\omega_{2}-\frac{\Omega}{W} \omega_{23}\right)\right] .
\end{aligned}
$$

Além disso, de (5.46), como

$$
\begin{aligned}
d e_{1}^{0}= & {\left[-\left(2 \operatorname{sen} \theta+\frac{\operatorname{sen}^{3} \theta}{\cos ^{2} \theta}\right) \frac{\Omega_{1}}{W} d \theta-\frac{\operatorname{sen}^{2} \theta}{\cos \theta}\left(d\left(\frac{\Omega_{1}}{W}\right)-\frac{\Omega_{2}}{W} \omega_{12}-\frac{\cos ^{2} \theta}{\operatorname{sen}^{2} \theta} \omega_{13}\right)\right] e_{1} } \\
& +\left[-\left(2 \operatorname{sen} \theta+\frac{\operatorname{sen}^{3} \theta}{\cos ^{2} \theta}\right) \frac{\Omega_{2}}{W} d \theta-\frac{\operatorname{sen}^{2} \theta}{\cos \theta}\left(\frac{\Omega_{1}}{W} \omega_{12}+d\left(\frac{\Omega_{2}}{W}\right)-\frac{\cos ^{2} \theta}{\operatorname{sen}^{2} \theta} \omega_{23}\right)\right] e_{2} \\
& +\left[-\left(2 \operatorname{sen} \theta+\frac{\operatorname{sen}^{3} \theta}{\cos ^{2} \theta}\right) \frac{\cos ^{2} \theta}{\operatorname{sen}^{2} \theta} d \theta-\frac{\operatorname{sen}^{2} \theta}{\cos \theta}\left(\frac{\Omega_{1}}{W} \omega_{13}+\frac{\Omega_{2}}{W} \omega_{23}+d\left(\frac{\cos ^{2} \theta}{\operatorname{sen}^{2} \theta}\right)\right)\right] e_{3},
\end{aligned}
$$




$$
\begin{aligned}
\omega_{12}^{0}=\langle & \left.d e_{1}^{0}, e_{2}^{0}\right\rangle \\
= & -\left(2 \operatorname{sen} \theta+\frac{\operatorname{sen}^{3} \theta}{\cos ^{2} \theta}\right) \frac{\Omega_{1}}{W} d \theta-\frac{\operatorname{sen}^{2} \theta}{\cos \theta}\left(\frac{1}{W}\left(\Omega_{2} \omega_{12}+c \Omega \omega_{1}-W\left(\frac{c}{K}+1\right) \omega_{13}\right)\right. \\
& \left.\left.-\frac{\Omega_{1}}{W^{2}} d W-\frac{\Omega_{2}}{W} \omega_{12}-\frac{\cos ^{2} \theta}{\operatorname{sen}^{2} \theta} \omega_{13}\right)\right]\left(\frac{\operatorname{sen} \theta}{\cos \theta} \frac{\Omega_{2}}{W}\right) \\
+ & {\left[-\left(2 \operatorname{sen} \theta+\frac{\operatorname{sen}^{3} \theta}{\cos ^{2} \theta}\right) \frac{\Omega_{2}}{W} d \theta-\frac{\operatorname{sen}^{2} \theta}{\cos \theta}\left(\frac{1}{W}\left(-\Omega_{1} \omega_{12}+c \Omega \omega_{2}-W\left(\frac{c}{K}+1\right) \omega_{23}\right)\right.\right.} \\
& \left.\left.+\frac{\Omega_{1}}{W} \omega_{12}-\frac{\Omega_{2}}{W^{2}} d W-\frac{\cos ^{2} \theta}{\operatorname{sen}^{2} \theta} \omega_{23}\right)\right]\left(-\frac{\operatorname{sen} \theta}{\cos \theta} \frac{\Omega_{1}}{W}\right) \\
= & -c \frac{\Omega}{W} \frac{\operatorname{sen}^{3} \theta}{\cos ^{2} \theta}\left[\frac{\Omega_{2}}{W}\left(\omega_{1}-\frac{\Omega}{W} \omega_{13}\right)-\frac{\Omega_{1}}{W}\left(\omega_{2}-\frac{\Omega}{W} \omega_{23}\right)\right],
\end{aligned}
$$

isto é,

$$
\omega_{12}^{0}=-c \frac{\Omega}{W} \frac{\operatorname{sen}^{2} \theta}{\cos \theta} \omega_{2}^{0}
$$

Usando (5.48) e (5.49), resulta

$$
\begin{aligned}
d \omega_{12}^{0} & =-c \frac{\operatorname{sen}^{2} \theta}{\cos \theta} d\left(\frac{\Omega}{W}\right) \wedge \omega_{2}^{0}-c \frac{\Omega}{W} d\left(\frac{\operatorname{sen}^{2} \theta}{\cos \theta}\right) \wedge \omega_{2}^{0}-c \frac{\Omega}{W} \frac{\operatorname{sen}^{2} \theta}{\cos \theta} d \omega_{2}^{0} \\
& =-c\left[-\operatorname{sen}^{2} \theta+2 \frac{\Omega^{2}}{W^{2}} c \operatorname{sen}^{4} \theta+c \frac{\Omega^{2}}{W^{2}} \frac{\operatorname{sen}^{6} \theta}{\cos ^{2} \theta}-c \frac{\Omega^{2}}{W^{2}} \frac{\operatorname{sen}^{4} \theta}{\cos ^{2} \theta}\right] \omega_{1}^{0} \wedge \omega_{2}^{0} \\
& =-c \operatorname{sen}^{2} \theta\left[c \frac{\Omega^{2}}{W^{2}} \operatorname{sen}^{2} \theta-1\right] \omega_{1}^{0} \wedge \omega_{2}^{0} .
\end{aligned}
$$

Logo, da equação de Gauss, $d \omega_{12}^{0}=-K_{0} \omega_{1}^{0} \wedge \omega_{2}^{0}$, a curvatura gaussiana de $X_{0}$ é

$$
K_{0}=c \operatorname{sen}^{2} \theta\left(c \frac{\Omega^{2}}{W^{2}} \operatorname{sen}^{2} \theta-1\right)
$$

Como, de (5.46), temos

$$
\cos \theta e_{1}^{0}-\operatorname{sen} \theta e_{3}^{0}=-e_{3},
$$

(5.42) se reduz a

$$
X=X_{0}+\frac{\Omega}{W}\left(\cos \theta e_{1}^{0}-\operatorname{sen} \theta e_{3}^{0}\right),
$$

que é, por construção, uma congruência de retas normal a $X$. Além disso, usando as notações

$$
\begin{aligned}
& T=\frac{\Omega}{W}, \\
& \omega_{12}^{0}=A \omega_{1}^{0}+B \omega_{2}^{0}, \\
& d \theta=\theta_{1}^{0} \omega_{1}^{0}+\theta_{2}^{0} \omega_{2}^{0},
\end{aligned}
$$

de (5.48) e (5.49) segue que

$$
\begin{aligned}
& c T \operatorname{sen}^{3} \theta-\theta_{1}^{0}=0, \\
& A=\theta_{2}^{0}=0, \\
& B \cos \theta+c T \operatorname{sen}^{2} \theta=0
\end{aligned}
$$

e, portanto,

$$
B \operatorname{sen} \theta \cos \theta+\theta_{1}^{0}=0 .
$$


Decorre também de (5.45) que

$$
\frac{1}{c \operatorname{sen}^{2} \theta}=\frac{T^{2} K-1}{K}
$$

de onde, por (5.52), obtemos

$$
\left(T^{2} K-1\right) \theta_{1}^{0}-T K \operatorname{sen} \theta=0 .
$$

Usando, agora, (5.52) em (5.50), segue que

$$
K_{0}=\frac{\theta_{1}^{0}}{T \operatorname{sen} \theta}\left(\frac{T \theta_{1}^{0}-\operatorname{sen} \theta}{\operatorname{sen} \theta}\right)
$$

isto é,

$$
T\left[\left(\theta_{1}^{0}\right)^{2}-K_{0} \operatorname{sen}^{2} \theta\right]-\theta_{1}^{0} \operatorname{sen} \theta=0 .
$$

Portanto, as relações de (4.46) a (4.49):

$$
\begin{aligned}
& B \operatorname{sen} \theta \cos \theta+\theta_{1}^{0}=0, \\
& A=\theta_{2}^{0}=0, \\
& \left(T^{2} K-1\right) \theta_{1}^{0}-T K \operatorname{sen} \theta=0, \\
& T\left[\left(\theta_{1}^{0}\right)^{2}-K_{0} \operatorname{sen}^{2} \theta\right]-\theta_{1}^{0} \operatorname{sen} \theta=0
\end{aligned}
$$

estão satisfeitas. Logo, da prova dos Teoremas 4.3.2 e 4.3.4, segue o resultado.

Corolário 5.3.4. A toda superfície de curvatura gaussiana constante $K$ não nula podemos associar uma familia a três parâmetros de superfícies de mesma curvatura gaussiana.

Demonstração. Sejam $X: U \subset \mathbb{R}^{2} \rightarrow \mathbb{R}^{3}$ a parametrização de uma superfície de curvatura gaussiana constante $K \neq 0$ e $\left\{e_{1}, e_{2}, e_{3}\right\}$ um triedro móvel principal associado a $X$. Do Teorema 5.3.3, existe uma família a três parâmetros de congruências de Guichard

$$
X=X_{0}-\frac{\Omega}{W} e_{3}=X_{0}+\frac{\Omega}{W}\left(\cos \theta e_{1}^{0}-\operatorname{sen} \theta e_{3}^{0}\right),
$$

sendo $X_{0}$ isométrica a um elipsóide de revolução ou a um hiperbolóide de revolução de duas folhas, se $K>0$, ou a uma das superfícies da Definição 4.3.3, se $K<0$. Então, do Corolário 4.2.11 e da Observação 4.3.5, a superfície

$$
X^{s}=X_{0}+\frac{\Omega}{W}\left(\cos \theta e_{1}^{0}+\operatorname{sen} \theta e_{3}^{0}\right),
$$

simétrica a $X$ em relação a $X_{0}$, possui também curvatura gaussiana constante $K^{s}=K \neq 0$.

Podemos reescrever (5.53) na forma

$$
X^{s}=X+2 \frac{\Omega}{W} \operatorname{sen} \theta e_{3}^{0}
$$

e, usando (5.45) e (5.46),

$$
\begin{aligned}
X^{s} & =X-2 \frac{\Omega}{W} \operatorname{sen}^{2} \theta\left(\frac{\Omega_{1}}{W} e_{1}+\frac{\Omega_{2}}{W} e_{2}-e_{3}\right) \\
& =X-\frac{2 K \Omega W}{c\left(K \Omega^{2}-W^{2}\right)}\left(\frac{\Omega_{1}}{W} e_{1}+\frac{\Omega_{2}}{W} e_{2}-e_{3}\right) .
\end{aligned}
$$


Portanto, usando a última relação de (5.41), vemos que

$$
X^{s}=X-\frac{2 \Omega W}{\Omega_{1}^{2}+\Omega_{2}^{2}+W^{2}}\left(\frac{\Omega_{1}}{W} e_{1}+\frac{\Omega_{2}}{W} e_{2}-e_{3}\right)
$$

é uma família a três parâmetros de superfícies de curvatura gaussiana $K^{s}=K \neq 0$.

Vamos terminar esta seção com uma observação que será útil nos resultados que seguem.

Observação 5.3.5. De (5.54) temos

$$
\begin{aligned}
d X^{s}= & d X-d\left(\frac{2 K \Omega W}{c\left(K \Omega^{2}-W^{2}\right)}\right)\left(\frac{\Omega_{1}}{W} e_{1}+\frac{\Omega_{2}}{W} e_{2}-e_{3}\right) \\
& -\frac{2 K \Omega W}{c\left(K \Omega^{2}-W^{2}\right)}\left[d\left(\frac{\Omega_{1}}{W}\right) e_{1}+\frac{\Omega_{1}}{W} d e_{1}+d\left(\frac{\Omega_{2}}{W}\right) e_{2}+\frac{\Omega_{2}}{W} d e_{2}-d e_{3}\right],
\end{aligned}
$$

de onde, usando as relações (5.41), obtemos

$$
\begin{aligned}
d X^{s}= & \frac{2 K \Omega_{1}\left[\left(\Omega^{2} K+W^{2}\right) \omega_{1}-2 \Omega W \omega_{13}\right]}{c\left(\Omega^{2} K-W^{2}\right)^{2}}\left[\left(\Omega_{1}-\frac{c\left(\Omega^{2} K-W^{2}\right)}{2 K \Omega_{1}}\right) e_{1}+\Omega_{2} e_{2}-W e_{3}\right] \\
& +\frac{2 K \Omega_{2}\left[\left(\Omega^{2} K+W^{2}\right) \omega_{2}-2 \Omega W \omega_{23}\right]}{c\left(\Omega^{2} K-W^{2}\right)^{2}}\left[\Omega_{1} e_{1}+\left(\Omega_{2}-\frac{c\left(\Omega^{2} K-W^{2}\right)}{2 K \Omega_{2}}\right) e_{2}-W e_{3}\right] .
\end{aligned}
$$

Portanto, $X^{s}$ é uma superfície parametrizada regular se, e somente se, as 1-formas diferenciais $\left[\left(\Omega^{2} K+W^{2}\right) \omega_{1}-2 \Omega W \omega_{13}\right]$ e $\left[\left(\Omega^{2} K+W^{2}\right) \omega_{2}-2 \Omega W \omega_{23}\right]$ são linearmente independentes, isto é, como $\omega_{13}=\kappa_{1} \omega_{1}$ e $\omega_{23}=\kappa_{2} \omega_{2}$, a expressão

$$
\left(\Omega^{2} K+W^{2}\right)^{2}-2 \Omega W\left(\Omega^{2} K+W^{2}\right)\left(\kappa_{1}+\kappa_{2}\right)+4 \Omega^{2} W^{2} \kappa_{1} \kappa_{2}
$$

deve ser nula. Denotando por $\alpha=\Omega^{2} K+W^{2}$, vamos agora analisar a seguinte situação:

$$
\alpha^{2}-2 \Omega W\left(\kappa_{1}+\kappa_{2}\right) \alpha+4 \Omega^{2} W^{2} \kappa_{1} \kappa_{2}=0 .
$$

Como $\Delta=4 \Omega^{2} W^{2}\left(\kappa_{1}-\kappa_{2}\right)^{2}$, temos que

$$
\alpha=\Omega W\left(\kappa_{1}+\kappa_{2}\right) \pm \Omega W\left(\kappa_{1}-\kappa_{2}\right)=2 \Omega W \kappa_{i}, \quad i=1,2,
$$

isto é,

$$
\kappa_{i}=\frac{\Omega^{2} K+W^{2}}{2 \Omega W} \quad i=1,2,
$$

anulam (5.56). Portanto, basta adicionar às condições iniciais que as curvaturas principais de $X$ em $q$ são diferentes de $\frac{\Omega^{2} K+W^{2}}{2 \Omega W}(q)$.

Segue da Proposição 1.3.8 que os campos

$$
\begin{aligned}
& {\left[\left(\Omega_{1}-\frac{c\left(\Omega^{2} K-W^{2}\right)}{2 K \Omega_{1}}\right) e_{1}+\Omega_{2} e_{2}-W e_{3}\right],} \\
& {\left[\Omega_{1} e_{1}+\left(\Omega_{2}-\frac{c\left(\Omega^{2} K-W^{2}\right)}{2 K \Omega_{2}}\right) e_{2}-W e_{3}\right]}
\end{aligned}
$$


são tangentes a $X^{s}$. Assim, podemos considerar os seguintes campos de vetores unitários

$$
\left\{\begin{array}{l}
e_{1}^{s}=\frac{2 K \Omega_{1}}{c\left(\Omega^{2} K-W^{2}\right)}\left[\left(\Omega_{1}-\frac{c\left(\Omega^{2} K-W^{2}\right)}{2 K \Omega_{1}}\right) e_{1}+\Omega_{2} e_{2}-W e_{3}\right] \\
e_{2}^{s}=\frac{2 K \Omega_{2}}{c\left(\Omega^{2} K-W^{2}\right)}\left[\Omega_{1} e_{1}+\left(\Omega_{2}-\frac{c\left(\Omega^{2} K-W^{2}\right)}{2 K \Omega_{2}}\right) e_{2}-W e_{3}\right] \\
e_{3}^{s}=\frac{2 K W}{c\left(\Omega^{2} K-W^{2}\right)}\left[-\Omega_{1} e_{2}-\Omega_{2} e_{2}+\left(W-\frac{c\left(\Omega^{2} K-W^{2}\right)}{2 K W}\right) e_{3}\right]
\end{array}\right.
$$

como triedro móvel associado a $X^{s}$. Usando este triedro, temos

$$
\begin{aligned}
d X^{s}= & \omega_{1}^{s} e_{1}^{s}+\omega_{2}^{s} e_{2}^{s} \\
= & \omega_{1}^{s} \frac{2 K \Omega_{1}}{c\left(\Omega^{2} K-W^{2}\right)}\left[\left(\Omega_{1}-\frac{c\left(\Omega^{2} K-W^{2}\right)}{2 K \Omega_{1}}\right) e_{1}+\Omega_{2} e_{2}-W e_{3}\right] \\
& +\omega_{2}^{s} \frac{2 K \Omega_{2}}{c\left(\Omega^{2} K-W^{2}\right)}\left[\Omega_{1} e_{1}+\left(\Omega_{2}-\frac{c\left(\Omega^{2} K-W^{2}\right)}{2 K \Omega_{2}}\right) e_{2}-W e_{3}\right]
\end{aligned}
$$

de onde, comparando com (5.55), obtemos

$$
\begin{aligned}
& \omega_{1}^{s}=\frac{1}{\Omega^{2} K-W^{2}}\left[\left(\Omega^{2} K+W^{2}\right) \omega_{1}-2 \Omega W \omega_{13}\right], \\
& \omega_{2}^{s}=\frac{1}{\Omega^{2} K-W^{2}}\left[\left(\Omega^{2} K+W^{2}\right) \omega_{2}-2 \Omega W \omega_{23}\right] .
\end{aligned}
$$

Agora, usando as relações (5.41) e (5.57), concluímos que

$$
\begin{aligned}
& \omega_{13}^{s}=-\frac{1}{\Omega^{2} K-W^{2}}\left[2 \Omega K W \omega_{1}-\left(\Omega^{2} K-W^{2}\right) \omega_{13}\right], \\
& \omega_{23}^{s}=-\frac{1}{\Omega^{2} K-W^{2}}\left[2 \Omega K W \omega_{2}-\left(\Omega^{2} K-W^{2}\right) \omega_{23}\right] .
\end{aligned}
$$

Terminamos esta seção observando que podemos obter resultados análogos a estes para superfícies de curvatura média constante não nula, já que estas são geometricamente equivalentes a superfícies de curvatura gaussiana constante positiva, pelo Teorema de Bonnet. Além disso, como conseqüência da Proposição 4.3.11, transformações de superfícies de mesma curvatura gaussiana ou média, obtidas nesta seção, preservam linhas de curvatura.

\subsection{Interpretação analítica}

Com base na associação entre superfícies mínimas e superfícies de curvatura gaussiana constante com soluções de equações diferenciais parciais, que foi estudada no Capítulo 2, vamos interpretar analiticamente os resultados vistos nas seções anteriores.

\subsubsection{Superfícies mínimas}

Seja $X: U \subset \mathbb{R}^{2} \rightarrow \mathbb{R}^{3}$ a parametrização principal de uma superfície mínima e $\left\{e_{1}, e_{2}, e_{3}\right\}$ o triedro móvel dado como na Proposição 1.3.3. Da prova da Proposição 2.1.1, podemos supor 
que a primeira e segunda formas quadráticas de $X$ são

$$
\begin{aligned}
\mathrm{I} & =e^{2 \phi}\left(d u^{2}+d v^{2}\right), \\
\mathrm{II} & =d u^{2}-d v^{2},
\end{aligned}
$$

onde $\phi$ é solução da equação de Liouville:

$$
\Delta \phi=e^{-2 \phi} .
$$

Neste caso, as 1-formas diferenciais associadas ao triedro móvel de $X$ são dadas por:

$$
\begin{aligned}
\omega_{1} & =e^{\phi} d u, \\
\omega_{2} & =e^{\phi} d v, \\
\omega_{12} & =-\phi_{v} d u+\phi_{u} d v, \\
\omega_{13} & =e^{-\phi} d u, \\
\omega_{23} & =-e^{-\phi} d v .
\end{aligned}
$$

Assim, reescrevemos o sistema (5.19) como:

$$
\left\{\begin{array}{l}
d \Omega_{1}=\left(-\Omega_{2} \phi_{v}+c W e^{\phi}+(c \Omega-W) e^{-\phi}\right) d u+\Omega_{2} \phi_{u} d v \\
d \Omega_{2}=\Omega_{1} \phi_{v} d u+\left(-\Omega_{1} \phi_{u}+c W e^{\phi}+(c \Omega-W) e^{-\phi}\right) d v \\
d W=e^{-\phi}\left(\Omega_{1} d u-\Omega_{2} d v\right) \\
d \Omega=e^{\phi}\left(\Omega_{1} d u+\Omega_{2} d v\right) \\
\Omega_{1}^{2}+\Omega_{2}^{2}+W^{2}-2 c \Omega W=0,
\end{array}\right.
$$

onde $c$ é uma constante arbitrária não nula.

Note que o Lema 5.2.2 é equivalente ao que segue.

Lema 5.4.1. Seja $\phi(u, v)$ uma solução de

$$
\Delta \phi=e^{-2 \phi} .
$$

Então, para cada constante c não nula, fixadas condições iniciais satisfazendo a última equação de (5.60), este sistema admite solução.

Demonstração. Considerando $\phi \neq 0$ uma solução de (5.61), podemos associar a $\phi$ uma superfície mínima $X$, cujas formas quadráticas são dadas por:

$$
\begin{aligned}
\mathrm{I} & =e^{2 \phi}\left(d u^{2}+d v^{2}\right), \\
\mathrm{II} & =d u^{2}-d v^{2} .
\end{aligned}
$$

Logo, as curvas coordenadas de $X$ são linhas de curvatura e, assim, segue do Lema 5.2.2 que, fixadas condições iniciais tais que satisfazem a última equação de (5.60), o sistema (5.19) admite solução. Como o sistema (5.19) equivale ao sistema (5.60), segue o resultado.

Conseqüentemente, o Corolário 5.2.4 é equivalente ao seguinte 
Teorema 5.4.2. Se $\phi(u, v)$ é solução de (5.61) então, fixada a constante $c \neq 0$, para cada solução não nula $\Omega, \Omega_{1}, \Omega_{2}, W$ do sistema (5.60), obtemos uma nova solução $\bar{\phi}$ de (5.61) definida por:

$$
e^{\bar{\phi}}=\frac{|c|}{c} \frac{\Omega}{W} e^{-\phi} .
$$

Demonstração. Provaremos esse teorema geometricamente usando os resultados da Seção 5.2.

Considere $X(u, v)$ uma parametrização da superfície mínima associada a $\phi$ (Corolário 2.1.3), cujas formas quadráticas são:

$$
\begin{aligned}
\mathrm{I} & =e^{2 \phi}\left(d u^{2}+d v^{2}\right), \\
\mathrm{II} & =d u^{2}-d v^{2} .
\end{aligned}
$$

Do Corolário 5.2.4 (equação (5.35)), cada solução não nula do sistema (5.60) determina uma superfície mínima $X^{s}$ que, pela Observação 5.2 .5 e por (5.59), possui 1-formas diferenciais

$$
\begin{aligned}
\omega_{1}^{s} & =\frac{\Omega}{W} e^{-\phi} d u, \\
\omega_{2}^{s} & =-\frac{\Omega}{W} e^{-\phi} d v, \\
\omega_{13}^{s} & =-\frac{W}{\Omega} e^{\phi} d u, \\
\omega_{23}^{s} & =-\frac{W}{\Omega} e^{\phi} d v .
\end{aligned}
$$

Assim, as formas quadráticas de $X^{s}$ são dadas por

$$
\begin{aligned}
\mathrm{I}^{s} & =\frac{\Omega^{2}}{W^{2}} e^{-2 \phi}\left(d u^{2}+d v^{2}\right), \\
\mathrm{II}^{s} & =-d u^{2}+d v^{2} .
\end{aligned}
$$

Note que, da última relação de (5.60), temos

$$
\Omega W=\frac{1}{2 c}\left(\Omega_{1}^{2}+\Omega_{2}^{2}+W^{2}\right)>0
$$

se, e somente se, $c>0$. Portanto, podemos definir a função $\bar{\phi}$ por

$$
e^{\bar{\phi}}=\frac{|c|}{c} \frac{\Omega}{W} e^{-\phi}
$$

de onde concluímos que $\bar{\phi}$ é solução de (5.61). Com efeito (repetindo o mesmo raciocínio visto na prova da Proposição 2.1.1) a curvatura gaussiana de $X^{s}$ é

$$
K^{s}=\frac{e_{s} g_{s}}{E_{s} G_{s}}=-e^{-4 \bar{\phi}} .
$$

Por outro lado, segue da equação de Gauss que

$$
K^{s}=-\frac{1}{\sqrt{E_{s} G_{s}}}\left[\left(\frac{\left(\sqrt{E_{s}}\right)_{v}}{\sqrt{G_{s}}}\right)_{v}+\left(\frac{\left(\sqrt{G_{s}}\right)_{u}}{\sqrt{E_{s}}}\right)_{u}\right]=-e^{-2 \bar{\phi}} \Delta \bar{\phi}
$$

Logo, obtemos

$$
\Delta \bar{\phi}=e^{-2 \bar{\phi}} .
$$




\subsubsection{Superfícies de curvatura gaussiana constante não nula}

De maneira análoga, vamos interpretar analiticamente os resultados obtidos na Seção 5.3 para superfícies de curvatura gaussiana constante $K \neq 0$.

\section{O caso $K>0$}

Sejam $X(u, v)$ a parametrização principal de uma superfície de curvatura gaussiana constante $K>0$ e $\left\{e_{1}, e_{2}, e_{3}\right\}$ o triedro móvel associado a $X$ como na Proposição 1.3.3. Da prova da Proposição 2.2.1, podemos supor que as formas quadráticas de $X$ são dadas por:

$$
\begin{aligned}
\mathrm{I} & =\frac{1}{K}\left(\operatorname{senh}^{2} \phi d u^{2}+\cosh ^{2} \phi d v^{2}\right), \\
\mathrm{II} & =\frac{1}{\sqrt{K}} \operatorname{senh} \phi \cosh \phi\left(d u^{2}+d v^{2}\right),
\end{aligned}
$$

onde a função $\phi$ é solução da equação de Sine-Gordon elíptica:

$$
\Delta \phi=-\operatorname{senh} \phi \cosh \phi .
$$

Além disso, temos que

$$
\begin{aligned}
\omega_{1} & =\frac{1}{\sqrt{K}} \operatorname{senh} \phi d u, \\
\omega_{2} & =\frac{1}{\sqrt{K}} \cosh \phi d v, \\
\omega_{12} & =-\phi_{v} d u+\phi_{u} d v, \\
\omega_{13} & =\cosh \phi d u, \\
\omega_{23} & =\operatorname{senh} \phi d v .
\end{aligned}
$$

Então, o sistema (5.41) é equivalente ao seguinte sistema linear e homogêneo:

$$
\left\{\begin{array}{l}
d \Omega_{1}=\left[-\Omega_{2} \phi_{v}+\frac{c}{\sqrt{K}} \Omega \operatorname{senh} \phi-W\left(\frac{c}{K}+1\right) \cosh \phi\right] d u+\Omega_{2} \phi_{u} d v \\
d \Omega_{2}=\Omega_{1} \phi_{v} d u+\left[-\Omega_{1} \phi_{u}+\frac{c}{\sqrt{K}} \Omega \cosh \phi-W\left(\frac{c}{K}+1\right) \operatorname{senh} \phi\right] d v \\
d W=\Omega_{1} \cosh \phi d u+\Omega_{2} \operatorname{senh} \phi d v \\
d \Omega=\frac{1}{\sqrt{K}}\left(\Omega_{1} \operatorname{senh} \phi d u+\Omega_{2} \cosh \phi d v\right) \\
\Omega_{1}^{2}+\Omega_{2}^{2}+W^{2}-c\left(\Omega^{2}-\frac{W^{2}}{K}\right)=0
\end{array}\right.
$$

onde $c \neq 0$ e $K>0$ são constantes arbitrárias.

Contudo, considerando $K>0$, o Lema 5.3.2 equivale ao

Lema 5.4.3. Seja $\phi$ solução da equação de Sine-Gordon elíptica:

$$
\Delta \phi=-\operatorname{senh} \phi \cosh \phi .
$$

Então, fixadas as constantes $c \neq 0$ e $K>0$, escolhendo condições iniciais sobre $\Omega, \Omega_{1}, \Omega_{2}, W$ satisfazendo a última equação de (5.63), este sistema admite solução não nula. 
Demonstração. Sejam $\phi$ uma solução não nula da equação de Sine-Gordon elíptica e $K>0$ uma constante fixada. Da Proposição 2.2.1, podemos associar a $\phi$ uma superfície $X$, de curvatura gaussiana igual a $K$, tal que:

$$
\begin{aligned}
\mathrm{I} & =\frac{1}{K}\left(\operatorname{senh}^{2} \phi d u^{2}+\cosh ^{2} \phi d v^{2}\right), \\
\mathrm{II} & =\frac{1}{\sqrt{K}} \operatorname{senh} \phi \cosh \phi\left(d u^{2}+d v^{2}\right) .
\end{aligned}
$$

Assim, do Lema 5.3.2, fixadas a constante $c \neq 0$ e condições iniciais sobre $\Omega, \Omega_{1}, \Omega_{2}, W$ satisfazendo a última relação de (5.41), este sistema admite solução. Como (5.41) equivale a (5.63), segue o resultado.

Conseqüentemente, o Corolário 5.3.4 é equivalente ao seguinte

Teorema 5.4.4. Se $\phi$ é solução da equação de Sine-Gordon elíptica então, fixadas as constantes $c \neq 0$ e $K>0$, para cada solução não nula $\Omega, \Omega_{1}, \Omega_{2}, W$ do sistema (5.63), obtemos uma nova solução $\bar{\phi}$ da equação (5.64) definida por:

$$
\begin{array}{ll}
\frac{\Omega^{2}}{W^{2}} K=\operatorname{coth}^{2}\left(\frac{\bar{\phi}-\phi}{2}\right), & \text { se } c>0, \\
\frac{\Omega^{2}}{W^{2}} K=\tanh ^{2}\left(\frac{\bar{\phi}-\phi}{2}\right), & \text { se } c<0 .
\end{array}
$$

Demonstração. Vamos apresentar a prova geométrica deste teorema, onde usando os resultados da Seção 5.3.

Sejam $\phi$ solução não nula da equação (5.64) e $X: U \subset \mathbb{R}^{2} \rightarrow \mathbb{R}^{3}$ a parametrização da superfície de curvatura gaussiana $K>0$ (associada a $\phi$ ) cujas formas quadráticas são:

$$
\begin{aligned}
\mathrm{I} & =\frac{1}{K}\left(\operatorname{senh}^{2} \phi d u^{2}+\cosh ^{2} \phi d v^{2}\right), \\
\mathrm{II} & =\frac{1}{\sqrt{K}} \operatorname{senh} \phi \cosh \phi\left(d u^{2}+d v^{2}\right) .
\end{aligned}
$$

Decorre do Corolário 5.3.4 que cada solução não nula $\Omega, \Omega_{1}, \Omega_{2}, W$ do sistema (5.63) determina uma superfície $X^{s}$ de mesma curvatura gaussiana $K$. Pela Observação 5.3.5, as 1-formas diferenciais de $X^{s}$ são dadas por:

$$
\begin{aligned}
\omega_{1}^{s} & =\frac{1}{\Omega^{2} K-W^{2}}\left(\left(\Omega^{2} K+W^{2}\right) \frac{1}{\sqrt{K}} \operatorname{senh} \phi-2 \Omega W \cosh \phi\right) d u, \\
\omega_{2}^{s} & =\frac{1}{\Omega^{2} K-W^{2}}\left(\left(\Omega^{2} K+W^{2}\right) \frac{1}{\sqrt{K}} \cosh \phi-2 \Omega W \operatorname{senh} \phi\right) d v, \\
\omega_{13}^{s} & =-\frac{1}{\Omega^{2} K-W^{2}}\left(2 \sqrt{K} \Omega W \operatorname{senh} \phi-\left(\Omega^{2} K+W^{2}\right) \cosh \phi\right) d u, \\
\omega_{23}^{s} & =-\frac{1}{\Omega^{2} K-W^{2}}\left(2 \sqrt{K} \Omega W \cosh \phi-\left(\Omega^{2} K+W^{2}\right) \operatorname{senh} \phi\right) d v .
\end{aligned}
$$

Definindo a função $\bar{\phi}(u, v)$ por:

$$
\begin{aligned}
& \operatorname{senh} \bar{\phi}=\frac{|c|}{c} \frac{1}{\Omega^{2} K-W^{2}}\left[\left(\Omega^{2} K+W^{2}\right) \operatorname{senh} \phi-2 \sqrt{K} \Omega W \cosh \phi\right], \\
& \cosh \bar{\phi}=\frac{|c|}{c} \frac{1}{\Omega^{2} K-W^{2}}\left[\left(\Omega^{2} K+W^{2}\right) \cosh \phi-2 \sqrt{K} \Omega W \operatorname{senh} \phi\right],
\end{aligned}
$$


resulta que

$$
\begin{aligned}
\mathrm{I}^{s} & =\left(\omega_{1}^{s}\right)^{2}+\left(\omega_{2}^{s}\right)^{2}=\frac{1}{K}\left(\operatorname{senh}^{2} \bar{\phi} d u^{2}+\cosh ^{2} \bar{\phi} d v^{2}\right), \\
\mathrm{II}^{s} & =\omega_{1}^{s} \omega_{13}^{s}+\omega_{2}^{s} \omega_{23}^{s}=\frac{1}{\sqrt{K}} \operatorname{senh} \bar{\phi} \cosh \bar{\phi}\left(d u^{2}+d v^{2}\right) .
\end{aligned}
$$

Observe que a função $\bar{\phi}$ está bem definida, pois da última relação de (5.63), temos que

$$
\Omega^{2} K-W^{2}=\frac{K}{c}\left(\Omega_{1}^{2}+\Omega_{2}^{2}+W^{2}\right)>0
$$

se, e somente se, $c>0$, de onde resulta:

$$
\begin{aligned}
\cosh \bar{\phi} & =\frac{|c|}{c} \frac{1}{\Omega^{2} K-W^{2}}\left[\left(\Omega^{2} K+W^{2}\right) \frac{e^{\phi}+e^{-\phi}}{2}-2 \sqrt{K} \Omega W \frac{e^{\phi}-e^{-\phi}}{2}\right] \\
& =\frac{|c|}{c} \frac{1}{\Omega^{2} K-W^{2}}\left[\frac{e^{\phi}}{2}(\Omega \sqrt{K}-W)^{2}+\frac{e^{-\phi}}{2}(\Omega \sqrt{K}+W)^{2}\right]>0
\end{aligned}
$$

e, também,

$$
\begin{aligned}
\cosh ^{2} \bar{\phi}-\operatorname{senh}^{2} \bar{\phi}= & \frac{1}{\left(\Omega^{2} K-W^{2}\right)^{2}}\left[\left(\left(\Omega^{2} K+W^{2}\right) \cosh \phi-2 \sqrt{K} \Omega W \operatorname{senh} \phi\right)^{2}\right. \\
& \left.-\left(\left(\Omega^{2} K+W^{2}\right) \operatorname{senh} \phi-2 \sqrt{K} \Omega W \cosh \phi\right)^{2}\right] \\
= & \frac{1}{\left(\Omega^{2} K-W^{2}\right)^{2}}\left[\Omega^{4} K^{2}-2 \Omega^{2} K W^{2}+W^{4}\right]=1 .
\end{aligned}
$$

Além disso,

$$
\cosh (\bar{\phi}-\phi)=\cosh \bar{\phi} \cosh \phi-\operatorname{senh} \bar{\phi} \operatorname{senh} \phi=\frac{|c|}{c} \frac{\Omega^{2} K+W^{2}}{\Omega^{2} K-W^{2}}
$$

isto é,

$$
\frac{\Omega^{2}}{W^{2}} K=\frac{\cosh (\bar{\phi}-\phi)+\frac{|c|}{c}}{\cosh (\bar{\phi}-\phi)-\frac{|c|}{c}} .
$$

Portanto, usando a relação trigonométrica

$$
\tanh ^{2}\left(\frac{a-b}{2}\right)=\frac{\cosh (a-b)-1}{\cosh (a-b)+1}
$$

resulta que, se $c>0$,

$$
\frac{\Omega^{2}}{W^{2}} K=\operatorname{coth}^{2}\left(\frac{\bar{\phi}-\phi}{2}\right)
$$

e se $c<0$,

$$
\frac{\Omega^{2}}{W^{2}} K=\tanh ^{2}\left(\frac{\bar{\phi}-\phi}{2}\right) .
$$

Como a superfície $X^{s}$ tem curvatura gaussiana $K^{s}=K$, segue da equação de Gauss que

$$
\Delta \bar{\phi}=-\operatorname{senh} \bar{\phi} \cosh \bar{\phi},
$$

ou seja, $\bar{\phi}$ é solução de (5.64). 


\section{O caso $K<0$}

Seja $X: U \subset \mathbb{R}^{2} \rightarrow \mathbb{R}^{3}$ a parametrização principal de uma superfície pseudo-esférica de curvatura $K$ e $\left\{e_{1}, e_{2}, e_{3}\right\}$ o triedro móvel dado como na Proposição 1.3.3. Pela prova da Proposição 2.3.3, podemos considerar as formas quadráticas de $X$ dadas por:

$$
\begin{aligned}
\mathrm{I} & =-\frac{1}{K}\left(\cos ^{2} \phi d u^{2}+\operatorname{sen}^{2} \phi d v^{2}\right), \\
\mathrm{II} & =\frac{1}{\sqrt{-K}} \operatorname{sen} \phi \cos \phi\left(d u^{2}-d v^{2}\right),
\end{aligned}
$$

onde $\phi$ é uma solução da equação de Sine-Gordon

$$
\phi_{u u}-\phi_{v v}=\operatorname{sen} \phi \cos \phi
$$

e

$$
\begin{aligned}
\omega_{1} & =\frac{1}{\sqrt{-K}} \cos \phi d u, \\
\omega_{2} & =\frac{1}{\sqrt{-K}} \operatorname{sen} \phi d v, \\
\omega_{12} & =\phi_{v} d u+\phi_{u} d v, \\
\omega_{13} & =\operatorname{sen} \phi d u, \\
\omega_{23} & =-\cos \phi d v .
\end{aligned}
$$

Assim, o sistema (5.41) pode ser escrito na forma:

$$
\left\{\begin{array}{l}
d \Omega_{1}=\left[\Omega_{2} \phi_{v}+\frac{c}{\sqrt{-K}} \Omega \cos \phi-W\left(\frac{c}{K}+1\right) \operatorname{sen} \phi\right] d u+\Omega_{2} \phi_{u} d v \\
d \Omega_{2}=-\Omega_{1} \phi_{v} d u+\left[-\Omega_{1} \phi_{u}+\frac{c}{\sqrt{-K}} \Omega \operatorname{sen} \phi+W\left(\frac{c}{K}+1\right) \cos \phi\right] d v \\
d W=\Omega_{1} \operatorname{sen} \phi d u-\Omega_{2} \cos \phi d v \\
d \Omega=\frac{1}{\sqrt{-K}}\left(\Omega_{1} \cos \phi d u+\Omega_{2} \operatorname{sen} \phi d v\right) \\
\Omega_{1}^{2}+\Omega_{2}^{2}+W^{2}-c\left(\Omega^{2}-\frac{W^{2}}{K}\right)=0
\end{array}\right.
$$

onde $c>0$ e $K<0$ são constantes arbitrárias.

Como feito para o caso de superfícies de curvatura gaussiana positiva, segue do Lema 5.3.2 o seguinte

Lema 5.4.5. Se ф é uma solução da equação de Sine-Gordon

$$
\phi_{u u}-\phi_{v v}=\operatorname{sen} \phi \cos \phi
$$

então, para constantes $c>0, K<0$ e fixadas condições iniciais sobre $\Omega, \Omega_{1}, \Omega_{2}, W$ satisfazendo a última relação de (5.66), este sistema admite solução.

Demonstração. Nessas condições, segue da Proposição 2.3.3 que podemos associar a $\phi$ uma superfície $X$ pseudo-esférica, de curvatura gaussiana igual a $K$, cujas formas quadráticas são dadas por:

$$
\begin{aligned}
\mathrm{I} & =-\frac{1}{K}\left(\cos ^{2} \phi d u^{2}+\operatorname{sen}^{2} \phi d v^{2}\right), \\
\mathrm{II} & =\frac{1}{\sqrt{-K}} \operatorname{sen} \phi \cos \phi\left(d u^{2}-d v^{2}\right) .
\end{aligned}
$$


Assim, fixadas condições iniciais sobre $\Omega, \Omega_{1}, \Omega_{2}, W$ satisfazendo a última relação de (5.41), do Lema 5.3.2, temos que o sistema (5.41) admite solução. Como o sistema (5.41) equivale a (5.66), segue o resultado.

Do Corolário 5.3.4 segue o seguinte

Teorema 5.4.6. Se $\phi$ é solução da equação de Sine-Gordon então, fixadas as constantes $K<0$ e c $>0$, para cada solução não nula $\Omega, \Omega_{1}, \Omega_{2}, W$ do sistema (5.66), obtemos uma nova solução $\bar{\phi}$ da equação de Sine-Gordon definida por

$$
-\frac{\Omega^{2}}{W^{2}} K=\cot ^{2}\left(\frac{\bar{\phi}-\phi}{2}\right) .
$$

Demonstração. Provaremos este resultado geometricamente.

Sejam $\phi$ solução não nula da equação de Sine-Gordon e $X(u, v)$ a parametrização principal da superfície de curvatura gaussiana constante $K<0$, cujas formas quadráticas são

$$
\begin{aligned}
\mathrm{I} & =-\frac{1}{K}\left(\cos ^{2} \phi d u^{2}+\operatorname{sen}^{2} \phi d v^{2}\right), \\
\mathrm{II} & =\frac{1}{\sqrt{-K}} \operatorname{sen} \phi \cos \phi\left(d u^{2}-d v^{2}\right) .
\end{aligned}
$$

Decorre do Corolário 5.3.4 que cada solução de (5.66) determina uma superfície $X^{s}$ de mesma curvatura gaussiana constante $K^{s}=K<0$ e que possui, pela Observação 5.3.5, 1-formas diferenciais dadas por:

$$
\begin{aligned}
\omega_{1}^{s} & =\frac{1}{\Omega^{2} K-W^{2}}\left(\left(\Omega^{2} K+W^{2}\right) \frac{1}{\sqrt{-K}} \cos \phi-2 \Omega W \operatorname{sen} \phi\right) d u, \\
\omega_{2}^{s} & =\frac{1}{\Omega^{2} K-W^{2}}\left(\left(\Omega^{2} K+W^{2}\right) \frac{1}{\sqrt{-K}} \operatorname{sen} \phi+2 \Omega W \cos \phi\right) d v, \\
\omega_{13}^{s} & =\frac{1}{\Omega^{2} K-W^{2}}\left(2 \sqrt{-K} \Omega W \cos \phi+\left(\Omega^{2} K+W^{2}\right) \operatorname{sen} \phi\right) d u, \\
\omega_{23}^{s} & =-\frac{1}{\Omega^{2} K-W^{2}}\left(-2 \sqrt{-K} \Omega W \operatorname{sen} \phi+\left(\Omega^{2} K+W^{2}\right) \cos \phi\right) d v .
\end{aligned}
$$

Definindo a função $\bar{\phi}(u, v)$ por

$$
\begin{aligned}
\cos \bar{\phi} & =\frac{1}{\Omega^{2} K-W^{2}}\left(\left(\Omega^{2} K+W^{2}\right) \cos \phi-2 \sqrt{-K} \Omega W \operatorname{sen} \phi\right), \\
\operatorname{sen} \bar{\phi} & =\frac{1}{\Omega^{2} K-W^{2}}\left(\left(\Omega^{2} K+W^{2}\right) \operatorname{sen} \phi+2 \sqrt{-K} \Omega W \cos \phi\right),
\end{aligned}
$$

o que é válido pois $\cos ^{2} \bar{\phi}+\operatorname{sen}^{2} \bar{\phi}=1$, as formas quadráticas de $X^{s}$ são dadas por:

$$
\begin{aligned}
\mathrm{I}^{s} & =\left(\omega_{1}^{s}\right)^{2}+\left(\omega_{2}^{s}\right)^{2}=-\frac{1}{K}\left(\cos ^{2} \bar{\phi} d u^{2}+\operatorname{sen}^{2} \bar{\phi} d v^{2}\right), \\
\mathrm{II}^{s} & =\omega_{1}^{s} \omega_{13}^{s}+\omega_{2}^{s} \omega_{23}^{s}=\frac{1}{\sqrt{-K}} \operatorname{sen} \bar{\phi} \cos \bar{\phi}\left(d u^{2}-d v^{2}\right) .
\end{aligned}
$$

Além disso, de

$$
\cos (\bar{\phi}-\phi)=\cos \bar{\phi} \cos \phi+\operatorname{sen} \bar{\phi} \operatorname{sen} \phi=\frac{\Omega^{2} K+W^{2}}{\Omega^{2} K-W^{2}}
$$


deduzimos que

$$
-\frac{\Omega^{2}}{W^{2}} K=\frac{1+\cos (\bar{\phi}-\phi)}{1-\cos (\bar{\phi}-\phi)}=\cot ^{2}\left(\frac{\bar{\phi}-\phi}{2}\right) .
$$

Como $X^{s}$ tem curvatura gaussiana $K^{s}=K<0$, segue da equação de Gauss que

$$
\bar{\phi}_{u u}-\bar{\phi}_{v v}=\operatorname{sen} \bar{\phi} \cos \bar{\phi}
$$

ou seja, $\bar{\phi}$ é solução da equação de Sine-Gordon.

\subsubsection{Composição de transformadas de Bäcklund dadas por Bianchi}

No Capítulo 3 desenvolvemos a teoria de Bäcklund para obter novas soluções das equações de Sine-Gordon e Sine-Gordon elíptica, a partir de uma dada solução. No caso da equação de Sine-Gordon elíptica vimos que, dada uma solução real, usando a transformação de Bäcklund complexa (3.49), obtemos uma outra solução real desta equação (Corolário 3.4.3). Também a partir de uma solução real da equação de Sine-Gordon, compondo transformações de Bäcklund (3.29), obtemos uma nova solução real desta equação, passando por soluções reais ou complexas (Teorema 3.3.6 e Observação 3.3.10).

Nesta seção vamos relacionar estes métodos com a teoria desenvolvida nas seções anteriores.

\section{O caso da equação de Sine-Gordon elíptica}

Considere $\phi$ uma solução real de (5.64) e $\theta \in \mathbb{R}$ uma constante não nula. Seja $\phi_{1}$ a solução de (5.64) associada a $\phi$ por $\tilde{B}\left(\theta_{1}\right)$, isto é, escrevendo $\phi_{1}=\omega+i \varphi$, esta é obtida integrando:

$$
\left\{\begin{array}{l}
\omega_{u}=\left(\operatorname{senh} \theta_{1} \cosh \phi \operatorname{senh} \omega+\cosh \theta_{1} \operatorname{senh} \phi \cosh \omega\right) \cos \varphi \\
\omega_{v}=\left(-\operatorname{senh} \theta_{1} \operatorname{senh} \phi \operatorname{senh} \omega-\cosh \theta_{1} \cosh \phi \cosh \omega\right) \operatorname{sen} \varphi
\end{array}\right.
$$

e

$$
\left\{\begin{array}{l}
\varphi_{u}+\phi_{v}=\left(\operatorname{senh} \theta_{1} \cosh \phi \cosh \omega+\cosh \theta_{1} \operatorname{senh} \phi \operatorname{senh} \omega\right) \operatorname{sen} \varphi \\
\varphi_{v}-\phi_{u}=\left(\operatorname{senh} \theta_{1} \operatorname{senh} \phi \cosh \omega+\cosh \theta_{1} \cosh \phi \operatorname{senh} \omega\right) \cos \varphi
\end{array}\right.
$$

Do Corolário 3.4.3, uma outra solução real $\phi^{*}$ de (5.64) é dada por:

$$
\tanh \frac{\phi^{*}-\phi}{2}=\tanh \theta_{1} \tanh \omega
$$

Definindo a função

$$
T=-\tanh \theta_{1} \tanh \omega
$$

e usando (5.67) e (5.68), iremos mostrar que $T$ satisfaz o sistema (5.40) para $c=-\cosh ^{2} \theta_{1}$ e $K=1$. De fato, calculando as derivadas parciais de $T$ com respeito a $u$ e $v$, obtemos:

$$
\begin{aligned}
T_{u} & =-\frac{\tanh \theta_{1}}{\cosh ^{2} \omega} \omega_{u} \\
& =-\left(\tanh \theta_{1} \tanh \omega \frac{\operatorname{senh} \theta_{1} \cosh \phi}{\cosh \omega}+\frac{\operatorname{senh} \theta_{1} \operatorname{senh} \phi}{\cosh \omega}\right) \cos \varphi \\
& =-(\operatorname{senh} \phi-T \cosh \phi) \frac{\operatorname{senh} \theta_{1} \cos \varphi}{\cosh \omega},
\end{aligned}
$$




$$
\begin{aligned}
T_{v} & =-\frac{\tanh \theta_{1}}{\cosh ^{2} \omega} \omega_{v} \\
& =\left(\tanh \theta_{1} \tanh \omega \frac{\operatorname{senh} \theta_{1} \operatorname{senh} \phi}{\cosh \omega}+\frac{\operatorname{senh} \theta_{1} \cosh \phi}{\cosh \omega}\right) \operatorname{sen} \varphi \\
& =(\cosh \phi-T \operatorname{senh} \phi) \frac{\operatorname{senh} \theta_{1} \operatorname{sen} \varphi}{\cosh \omega} .
\end{aligned}
$$

De (5.62), temos que

$$
d T=T_{1} \omega_{1}+T_{2} \omega_{2}=T_{1} \operatorname{senh} \phi d u+T_{2} \cosh \phi d v,
$$

de onde resulta:

$$
T_{1}=\frac{T_{u}}{\operatorname{senh} \phi}, \quad T_{2}=\frac{T_{v}}{\cosh \phi}
$$

Além disso, como $\omega_{13}=\kappa_{1} \omega_{1}$ e $\omega_{23}=\kappa_{2} \omega_{2}$, então:

$$
\kappa_{1}=\operatorname{coth} \phi, \quad \kappa_{2}=\tanh \phi .
$$

Assim, de (5.6) segue que

$$
\begin{aligned}
& F_{1}=\frac{T_{u}}{\operatorname{senh} \phi-T \cosh \phi}=-\frac{\operatorname{senh} \theta_{1} \cos \varphi}{\cosh \omega}, \\
& F_{2}=\frac{T_{v}}{\cosh \phi-T \operatorname{senh} \phi}=\frac{\operatorname{senh} \theta_{1} \operatorname{sen} \varphi}{\cosh \omega}
\end{aligned}
$$

de onde obtemos:

$$
F_{1}^{2}+F_{2}^{2}+1=\frac{\operatorname{senh}^{2} \theta_{1}}{\cosh ^{2} \omega}+1=-\cosh ^{2} \theta_{1}\left(-\frac{\tanh ^{2} \theta_{1}}{\cosh ^{2} \omega}-\frac{1}{\cosh ^{2} \theta_{1}}\right)=c\left(T^{2}-1\right)
$$

ou seja, a primeira equação de (5.40) está satisfeita. Falta ainda provar que

$$
\left\{\begin{aligned}
d F_{1}= & \left(-F_{2} \phi_{v}+F_{2}^{2} \cosh \phi-T \cosh ^{2} \theta_{1} \operatorname{senh} \phi+T^{2} \cosh ^{2} \theta_{1} \cosh \phi\right) d u \\
& +\left(F_{2} \phi_{u}-F_{1} F_{2} \operatorname{senh} \phi\right) d v \\
d F_{2}= & \left(F_{1} \phi_{v}-F_{1} F_{2} \cosh \phi\right) d u \\
& +\left(-F_{1} \phi_{u}+F_{1}^{2} \operatorname{senh} \phi-T \cosh ^{2} \theta_{1} \cosh \phi+T^{2} \cosh ^{2} \theta_{1} \operatorname{senh} \phi\right) d v
\end{aligned}\right.
$$

ou seja,

$$
\left\{\begin{array}{l}
\frac{\partial F_{1}}{\partial u}=-F_{2} \phi_{v}+F_{2}^{2} \cosh \phi-T \cosh ^{2} \theta_{1} \operatorname{senh} \phi+T^{2} \cosh ^{2} \theta_{1} \cosh \phi \\
\frac{\partial F_{1}}{\partial v}=F_{2} \phi_{u}-F_{1} F_{2} \operatorname{senh} \phi \\
\frac{\partial F_{2}}{\partial u}=F_{1} \phi_{v}-F_{1} F_{2} \cosh \phi \\
\frac{\partial F_{2}}{\partial v}=-F_{1} \phi_{u}+F_{1}^{2} \operatorname{senh} \phi-T \cosh ^{2} \theta_{1} \cosh \phi+T^{2} \cosh ^{2} \theta_{1} \operatorname{senh} \phi .
\end{array}\right.
$$

Provaremos apenas a primeira destas equações, pois as outras seguem de maneira análoga. 
Calculando a derivada parcial de $F_{1}$ com respeito a $u$, temos:

$$
\begin{aligned}
\frac{\partial F_{1}}{\partial u}= & \frac{\operatorname{senh} \theta_{1}}{\cosh \omega}\left[\operatorname{sen} \varphi \cosh \omega \varphi_{u}+\cos \varphi \operatorname{senh} \omega \omega_{u}\right] \\
= & \frac{\operatorname{senh} \theta_{1} \operatorname{sen} \varphi}{\cosh \omega}\left(-\phi_{v}+\frac{\operatorname{senh} \theta_{1}}{\cosh \omega} \cosh \phi \cosh ^{2} \omega \operatorname{sen} \varphi+\cosh \theta_{1} \operatorname{senh} \phi \operatorname{senh} \omega \operatorname{sen} \varphi\right) \\
& +\frac{\operatorname{senh} \theta_{1} \cos \varphi}{\cosh \omega} \tanh \omega\left(\operatorname{senh} \theta_{1} \cosh \phi \operatorname{senh} \omega \cos \varphi+\cosh \theta_{1} \operatorname{senh} \phi \cosh \omega \cos \varphi\right) \\
= & -F_{2} \phi_{v}+\frac{\operatorname{senh}^{2} \theta_{1} \operatorname{sen}^{2} \varphi}{\cosh ^{2} \omega} \cosh \phi \cosh ^{2} \omega-T \cosh ^{2} \theta_{1} \operatorname{senh} \phi \\
& +\tanh ^{2} \theta_{1} \tanh ^{2} \omega \cosh \theta_{1} \cosh \phi \cos ^{2} \varphi \\
= & -F_{2} \phi_{v}+F_{2}^{2} \cosh \phi-T \cosh ^{2} \theta_{1} \operatorname{senh} \phi+T^{2} \cosh ^{2} \theta_{1} \cosh \phi,
\end{aligned}
$$

onde na segunda igualdade usamos (5.67) e (5.68).

Note que, usando a relação $T=\frac{\Omega}{W}$ em (5.69), decorre do Teorema 5.4 .4 que a solução $\phi^{*}$ provém da teoria geométrica desenvolvida neste capítulo, isto é, $\phi$ e $\phi^{*}$ são soluções da equação de Sine-Gordon elíptica associadas a superfícies $X$ e $X^{s}$ de curvatura gaussiana $K=1$.

\section{O caso da equação de Sine-Gordon (Parte A)}

Para uma dada solução real $\phi$ da equação de Sine-Gordon, considere inicialmente as constantes reais não nulas $\theta_{1}$ e $\theta_{2}=\pi-\theta_{1}$. Sejam $\phi_{1}$ e $\phi_{2}$ as soluções associadas a $\phi$ respectivamente por $B\left(\theta_{1}\right)$ e $B\left(\theta_{2}\right)$, isto é, $\phi_{1}$ e $\phi_{2}$ são soluções das transformações de Bäcklund:

$$
\left\{\begin{array}{l}
\phi_{1 u}+\phi_{v}=\operatorname{sen} \phi_{1} \cos \phi \csc \theta_{1}+\cot \theta_{1} \cos \phi_{1} \operatorname{sen} \phi, \\
\phi_{1 v}+\phi_{u}=-\cos \phi_{1} \operatorname{sen} \phi \csc \theta_{1}-\cot \theta_{1} \operatorname{sen} \phi_{1} \cos \phi
\end{array}\right.
$$

e

$$
\left\{\begin{array}{l}
\phi_{2 u}+\phi_{v}=\operatorname{sen} \phi_{2} \cos \phi \csc \theta_{1}-\cot \theta_{1} \cos \phi_{2} \operatorname{sen} \phi \\
\phi_{2 v}+\phi_{u}=-\cos \phi_{2} \operatorname{sen} \phi \csc \theta_{1}+\cot \theta_{1} \operatorname{sen} \phi_{2} \cos \phi
\end{array}\right.
$$

Do Teorema de Permutabilidade 3.3.6, uma nova solução real $\phi^{*}$ da equação de Sine-Gordon será dada por:

$$
\tan \frac{\phi^{*}-\phi}{2}=\frac{1}{\cos \theta_{1}} \tan \frac{\phi_{1}-\phi_{2}}{2} .
$$

Definindo a função $T$ por:

$$
T=-\cos \theta_{1} \cot \frac{\phi_{1}-\phi_{2}}{2},
$$

segue de (5.71) e (5.72) que $T$ satisfaz o sistema (5.40), considerando $K=-1$ e $c=\frac{1}{\operatorname{sen}^{2} \theta_{1}}$. Com efeito, calculando as derivadas parciais de $T$ temos:

$$
\begin{aligned}
& T_{u}=\frac{1}{2} \frac{\cos \theta_{1}}{\operatorname{sen}^{2} \frac{\phi_{1}-\phi_{2}}{2}}\left(\phi_{1 u}-\phi_{2 u}\right) \\
& =\frac{\cot \theta_{1}}{\operatorname{sen} \frac{\phi_{1}-\phi_{2}}{2}}\left[\frac{\cos \phi}{\operatorname{sen} \frac{\phi_{1}-\phi_{2}}{2}} \frac{1}{2}\left(\operatorname{sen} \phi_{1}-\operatorname{sen} \phi_{2}\right)+\frac{\operatorname{sen} \phi \cos \theta_{1}}{\operatorname{sen} \frac{\phi_{1}-\phi_{2}}{2}} \frac{1}{2}\left(\cos \phi_{1}+\cos \phi_{2}\right)\right] \\
& =\frac{\cot \theta_{1} \cos \frac{\phi_{1}+\phi_{2}}{2}}{\operatorname{sen} \frac{\phi_{1}-\phi_{2}}{2}}(\cos \phi-T \operatorname{sen} \phi),
\end{aligned}
$$




$$
\begin{aligned}
T_{v} & =\frac{1}{2} \frac{\cos \theta_{1}}{\operatorname{sen}^{2} \frac{\phi_{1}-\phi_{2}}{2}}\left(\phi_{1 v}-\phi_{2 v}\right) \\
& =-\frac{\cot \theta_{1}}{\operatorname{sen} \frac{\phi_{1}-\phi_{2}}{2}}\left[\frac{\operatorname{sen} \phi}{\operatorname{sen} \frac{\phi_{1}-\phi_{2}}{2}} \frac{1}{2}\left(\cos \phi_{1}-\cos \phi_{2}\right)+\frac{\cos \phi \cos \theta_{1}}{\operatorname{sen} \frac{\phi_{1}-\phi_{2}}{2}} \frac{1}{2}\left(\operatorname{sen} \phi_{1}+\operatorname{sen} \phi_{2}\right)\right] \\
& =\frac{\cot \theta_{1} \operatorname{sen} \frac{\phi_{1}+\phi_{2}}{2}}{\operatorname{sen} \frac{\phi_{1}-\phi_{2}}{2}}(\operatorname{sen} \phi+T \cos \phi) .
\end{aligned}
$$

Como, de (5.65),

$$
d T=T_{1} \omega_{1}+T_{2} \omega_{2}=T_{1} \cos \phi d u+T_{2} \operatorname{sen} \phi d v,
$$

obtemos

$$
T_{1}=\frac{T_{u}}{\cos \phi}, \quad T_{2}=\frac{T_{v}}{\operatorname{sen} \phi}
$$

e, de $\omega_{13}=\kappa_{1} \omega_{1}$ e $\omega_{23}=\kappa_{2} \omega_{2}$, também temos

$$
\kappa_{1}=\tan \phi, \quad \kappa_{2}=-\cot \phi .
$$

Como de (5.6),

$$
\begin{gathered}
F_{1}=\frac{T_{u}}{\cos \phi-T \operatorname{sen} \phi}=\frac{\cot \theta_{1} \cos \frac{\phi_{1}+\phi_{2}}{2}}{\operatorname{sen} \frac{\phi_{1}-\phi_{2}}{2}}, \\
F_{2}=\frac{T_{v}}{\operatorname{sen} \phi+T \cos \phi}=\frac{\cot \theta_{1} \operatorname{sen} \frac{\phi_{1}+\phi_{2}}{2}}{\operatorname{sen} \frac{\phi_{1}-\phi_{2}}{2}},
\end{gathered}
$$

concluímos que

$$
F_{1}^{2}+F_{2}^{2}+1=\frac{\cot ^{2} \theta_{1}}{\operatorname{sen}^{2} \frac{\phi_{1}-\phi_{2}}{2}}+1=\frac{1}{\operatorname{sen}^{2} \theta_{1}}\left(\frac{\cos ^{2} \theta_{1}}{\operatorname{sen}^{2} \frac{\phi_{1}-\phi_{2}}{2}}+\operatorname{sen}^{2} \theta_{1}\right)=\frac{1}{\operatorname{sen}^{2} \theta_{1}}\left(T^{2}+1\right),
$$

ou seja, a primeira equação de (5.40) está satisfeita. Falta ainda mostrar que

$$
\left\{\begin{aligned}
d F_{1}= & \left(F_{2} \phi_{v}+F_{2}^{2} \operatorname{sen} \phi+\frac{1}{\operatorname{sen}^{2} \theta_{1}} T \cos \phi-\frac{1}{\operatorname{sen}^{2} \theta_{1}} T^{2} \operatorname{sen} \phi\right) d u \\
& +\left(F_{2} \phi_{u}+F_{1} F_{2} \cos \phi\right) d v \\
d F_{2}= & \left(-F_{1} \phi_{v}-F_{1} F_{2} \operatorname{sen} \phi\right) d u \\
& +\left(-F_{1} \phi_{u}-F_{1}^{2} \cos \phi+\frac{1}{\operatorname{sen}^{2} \theta_{1}} T \operatorname{sen} \phi-\frac{1}{\operatorname{sen}^{2} \theta_{1}} T^{2} \cos \phi\right) d v
\end{aligned}\right.
$$

ou seja,

$$
\left\{\begin{array}{l}
\frac{\partial F_{1}}{\partial u}=F_{2} \phi_{v}+F_{2}^{2} \operatorname{sen} \phi+\frac{1}{\operatorname{sen}^{2} \theta_{1}} T \cos \phi-\frac{1}{\operatorname{sen}^{2} \theta_{1}} T^{2} \operatorname{sen} \phi \\
\frac{\partial F_{1}}{\partial v}=F_{2} \phi_{u}+F_{1} F_{2} \cos \phi \\
\frac{\partial F_{2}}{\partial u}=-F_{1} \phi_{v}-F_{1} F_{2} \operatorname{sen} \phi \\
\frac{\partial F_{2}}{\partial v}=-F_{1} \phi_{u}-F_{1}^{2} \cos \phi+\frac{1}{\operatorname{sen}^{2} \theta_{1}} T \operatorname{sen} \phi-\frac{1}{\operatorname{sen}^{2} \theta_{1}} T^{2} \cos \phi
\end{array}\right.
$$


Mostraremos somente a primeira equação acima, pois as outras seguem da mesma maneira. Calculando a derivada parcial de $F_{1}$ com respeito a $u$, temos:

$$
\begin{aligned}
\frac{\partial F_{1}}{\partial u}= & \frac{1}{2} \frac{\cos \theta_{1}}{\operatorname{sen}^{2} \frac{\phi_{1}-\phi_{2}}{2}}\left[-\operatorname{sen} \frac{\phi_{1}+\phi_{2}}{2} \operatorname{sen} \frac{\phi_{1}-\phi_{2}}{2}\left(\phi_{1}+\phi_{2}\right)_{u}\right. \\
& \left.-\cos \frac{\phi_{1}+\phi_{2}}{2} \cos \frac{\phi_{1}-\phi_{2}}{2}\left(\phi_{1}-\phi_{2}\right)_{u}\right] \\
= & \frac{\cot \theta_{1}}{\operatorname{sen}^{2} \frac{\phi_{1}-\phi_{2}}{2}} \phi_{v} \frac{1}{2}\left(\cos \phi_{2}-\cos \phi_{1}\right)-\frac{\cos \theta_{1} \cos \phi}{\operatorname{sen}^{2} \theta_{1} \operatorname{sen}^{2} \frac{\phi_{1}-\phi_{2}}{2}} \frac{1}{2} \operatorname{sen}\left(\phi_{1}-\phi_{2}\right) \\
& -\frac{\cot ^{2} \theta_{1}}{\operatorname{sen}^{2} \frac{\phi_{1}-\phi_{2}}{2}} \operatorname{sen} \phi \cos \phi_{1} \cos \phi_{2} \\
= & F_{2} \phi_{v}-\frac{1}{\operatorname{sen}^{2} \theta_{1}} T \cos \phi+F_{2}^{2} \operatorname{sen} \phi-\frac{1}{\operatorname{sen}^{2} \theta_{1}} T^{2} \operatorname{sen} \phi,
\end{aligned}
$$

onde usamos (5.71) e (5.72) na segunda igualdade.

Usando $T=\frac{\Omega}{W}$, observamos que $\phi^{*}$ obtida por:

$$
\frac{\Omega^{2}}{W^{2}}=T^{2}=\cot ^{2} \frac{\phi^{*}-\phi}{2}
$$

é a solução resultante do Teorema 5.4.6, associada a uma superfície de curvatura gaussiana constante $K^{*}=-1$.

\section{O caso da equação de Sine-Gordon (Parte B)}

Se considerarmos uma solução real $\phi$ da equação de Sine-Gordon e as constantes $\theta_{1}=\frac{\pi}{2}-i \sigma$ e $\theta_{2}=\bar{\theta}_{1}$, pela Observação 2.1.2, as soluções $\phi_{1}=\omega+i \varphi$ e $\phi_{2}=\bar{\phi}_{1}$ associadas a $\phi$ por $B\left(\theta_{1}\right)$ e $B\left(\theta_{2}\right)$, respectivamente, são obtidas integrando os sistemas:

$$
\left\{\begin{array}{l}
\omega_{u}+\phi_{v}=\left(\cosh \varphi \cos \phi \frac{1}{\cosh \sigma}+\frac{\operatorname{senh} \sigma}{\cosh \sigma} \operatorname{senh} \varphi \operatorname{sen} \phi\right) \operatorname{sen} \omega \\
\omega_{v}+\phi_{u}=\left(-\cosh \varphi \operatorname{sen} \phi \frac{1}{\cosh \sigma}+\frac{\operatorname{senh} \sigma}{\cosh \sigma} \operatorname{senh} \varphi \cos \phi\right) \cos \omega
\end{array}\right.
$$

$\mathrm{e}$

$$
\left\{\begin{array}{l}
\varphi_{u}=\left(\operatorname{senh} \varphi \cos \phi \frac{1}{\cosh \sigma}+\frac{\operatorname{senh} \sigma}{\cosh \sigma} \cosh \varphi \operatorname{sen} \phi\right) \cos \omega \\
\varphi_{v}=\left(\operatorname{senh} \varphi \operatorname{sen} \phi \frac{1}{\cosh \sigma}-\frac{\operatorname{senh} \sigma}{\cosh \sigma} \cosh \varphi \cos \phi\right) \operatorname{sen} \omega
\end{array}\right.
$$

Decorre do Teorema de Permutabilidade 3.3.6 que a quarta solução da equação de Sine-Gordon é dada por:

$$
\tan \frac{\phi^{*}-\phi}{2}=\frac{\operatorname{sen} \frac{\pi}{2}}{\operatorname{sen}(i \sigma)} \tan (i \sigma)=\frac{1}{\operatorname{senh} \sigma} \tanh \varphi .
$$

Como feito para o caso anterior, definindo

$$
T=-\operatorname{senh} \sigma \operatorname{coth} \varphi
$$


e utilizando (5.73) e (5.74), podemos verificar que $T$ satisfaz o sistema (5.40) escrito como:

$$
\left\{\begin{aligned}
F_{1}^{2}+ & F_{2}^{2}+1=\frac{1}{\cosh ^{2} \sigma}\left(T^{2}+1\right) \\
d F_{1}= & \left(F_{2} \phi_{v}+\frac{1}{\cosh ^{2} \sigma} T \cos \phi+F_{2}^{2} \operatorname{sen} \phi-\frac{1}{\cosh ^{2} \sigma} T^{2} \operatorname{sen} \phi\right) d u \\
& +\left(F_{2} \phi_{u}+F_{1} F_{2} \cos \phi\right) d v \\
d F_{2}= & \left(-F_{1} \phi_{v}-F_{1} F_{2} \operatorname{sen} \phi\right) d u \\
& +\left(-F_{1} \phi_{u}+\frac{1}{\cosh ^{2} \sigma} T \operatorname{sen} \phi-F_{1}^{2} \cos \phi-\frac{1}{\cosh ^{2} \sigma} T^{2} \cos \phi\right) d v
\end{aligned}\right.
$$

onde estamos considerando $K=-1, c=\frac{1}{\cosh ^{2} \sigma}$ e as funções $F_{1}$ e $F_{2}$ são dadas por:

$$
\begin{aligned}
& F_{1}=\frac{T_{u}}{\cos \phi-T \operatorname{sen} \phi}=\frac{\tanh \sigma \cos \omega}{\operatorname{senh} \varphi}, \\
& F_{1}=\frac{T_{u}}{\operatorname{sen} \phi+T \cos \phi}=\frac{\tanh \sigma \operatorname{sen} \omega}{\operatorname{senh} \varphi},
\end{aligned}
$$

obtidas a partir de (5.6) e (5.75). Novamente, sendo $T=\frac{\Omega}{W}$, segue que $\phi^{*}$ definida como

$$
T^{2}=\cot ^{2} \frac{\phi^{*}-\phi}{2}
$$

é uma das soluções obtidas no Teorema 5.4.6.

\subsection{Superfícies de curvatura média constante não nula}

Até agora foi desenvolvida a teoria geométrica das transformações de superfícies mínimas e de superfícies de curvatura gaussiana constante não nula e a correspondente teoria analítica. Porém deixamos de desenvolver estas teorias para superfícies de curvatura média constante $\mathrm{H} \neq 0$, por serem geometricamente equivalentes às superfícies de curvatura gaussiana constante positiva (Teorema de Bonnet). Estas superfícies estão associadas, pela Proposição 2.1.1, a soluções da equação

$$
\Delta \phi=C^{2} e^{-2 \phi}-\mathrm{H}^{2} e^{2 \phi}
$$

onde $C$ é uma constante não nula.

Para obter resultados análogos aos anteriores para estas superfícies a idéia é, a partir de uma superfície $X$ de curvatura média constante $\mathrm{H} \neq 0$, aplicar o Teorema de Bonnet para obter uma superfície $\tilde{X}$ de curvatura gaussiana $\tilde{K}=4 \mathrm{H}^{2}$. Aplicando o Corolário 5.3.4 para $\tilde{X}$ se obtém uma família a três parâmetros de superfícies de mesma curvatura gaussiana. Considerando $\tilde{X}^{s}$ uma delas e aplicando novamente o Teorema de Bonnet, obteremos a superfície $X^{s}$, de curvatura média igual a $\mathrm{H}^{s}=\mathrm{H}$.

Seja $X(u, v)$ a parametrização principal de uma superfície de curvatura média constante $\mathrm{H} \neq 0$ e $\left\{e_{1}, e_{2}, e_{3}\right\}$ o triedro móvel associado a $X$ como dado na Proposição 1.3 .3 , sendo $e_{3}$ o campo normal a $X$ tal que sua curvatura média é igual a H. Da prova da Proposição 2.1.1, podemos supor que as formas quadráticas de $X$ sejam dadas por:

$$
\begin{aligned}
\mathrm{I} & =e^{2 \phi}\left(d u^{2}+d v^{2}\right), \\
\mathrm{II} & =\left(\mathrm{H} e^{2 \phi}+C\right) d u^{2}+\left(\mathrm{H} e^{2 \phi}-C\right) d v^{2},
\end{aligned}
$$


onde $C$ é uma constante não nula e $\phi$ é uma solução de (5.76). Neste caso, da Proposição 1.3.3 segue que as 1-formas associadas a $X$ são dadas por:

$$
\begin{aligned}
\omega_{1} & =e^{\phi} d u, \\
\omega_{2} & =e^{\phi} d v, \\
\omega_{12} & =-\phi_{v} d u+\phi_{u} d v, \\
\omega_{13} & =\left(\mathrm{H} e^{\phi}+C e^{-\phi}\right) d u, \\
\omega_{23} & =\left(\mathrm{H} e^{\phi}-C e^{-\phi}\right) d v .
\end{aligned}
$$

Seja $\tilde{X}$ a superfície paralela a $X$, de curvatura gaussiana $\tilde{K}=4 \mathrm{H}^{2}$, dada por

$$
\tilde{X}=X+\frac{1}{2 \mathrm{H}} e_{3}
$$

Considere o triedro móvel $\tilde{e}_{i}=e_{i}, i=1,2,3$, associado a $\tilde{X}$ e $\tilde{\omega}_{1}, \tilde{\omega}_{2}, \tilde{\omega}_{i j}, i, j=1,2,3$, as 1-formas associadas a este triedro. Segue da Proposição 3.1.7 que

$$
\begin{aligned}
\tilde{\omega}_{1} & =\omega_{1}-\frac{1}{2 \mathrm{H}} \omega_{13}=\frac{1}{2 \mathrm{H}}\left(\mathrm{H} e^{\phi}-C e^{-\phi}\right) d u, \\
\tilde{\omega}_{2} & =\omega_{2}-\frac{1}{2 \mathrm{H}} \omega_{23}=\frac{1}{2 \mathrm{H}}\left(\mathrm{H} e^{\phi}+C e^{-\phi}\right) d v, \\
\tilde{\omega}_{12} & =\omega_{12}=-\phi_{v} d u+\phi_{u} d v, \\
\tilde{\omega}_{13} & =\omega_{13}=\left(\mathrm{H} e^{\phi}+C e^{-\phi}\right) d u, \\
\tilde{\omega}_{23} & =\omega_{23}=\left(\mathrm{H} e^{\phi}-C e^{-\phi}\right) d v .
\end{aligned}
$$

Do Corolário 5.3.4, podemos obter uma nova superfície $\tilde{X}^{s}$ (associada a $\tilde{X}$ ) de curvatura gaussiana $\tilde{K}^{s}=\tilde{K}=4 \mathrm{H}^{2}$, integrando o sistema (5.41) escrito na forma:

$$
\left\{\begin{array}{l}
d \Omega_{1}=\left[-\Omega_{2} \phi_{v}+\frac{c}{2 \mathrm{H}} \Omega\left(\mathrm{H} e^{\phi}-C e^{-\phi}\right)-W\left(\frac{c}{4 \mathrm{H}^{2}}+1\right)\left(\mathrm{H} e^{\phi}+C e^{-\phi}\right)\right] d u+\Omega_{2} \phi_{u} d v \\
d \Omega_{2}=\Omega_{1} \phi_{v} d u+\left[-\Omega_{1} \phi_{u}+\frac{c}{2 \mathrm{H}} \Omega\left(\mathrm{H} e^{\phi}+C e^{-\phi}\right)-W\left(\frac{c}{4 \mathrm{H}^{2}}+1\right)\left(\mathrm{H} e^{\phi}-C e^{-\phi}\right)\right] d v \\
d W=\Omega_{1}\left(\mathrm{H} e^{\phi}+C e^{-\phi}\right) d u+\Omega_{2}\left(\mathrm{H} e^{\phi}-C e^{-\phi}\right) d v \\
d \Omega=\frac{\Omega_{1}}{2 \mathrm{H}}\left(\mathrm{H} e^{\phi}-C e^{-\phi}\right) d u+\frac{\Omega_{2}}{2 \mathrm{H}}\left(\mathrm{H} e^{\phi}+C e^{-\phi}\right) d v \\
\Omega_{1}^{2}+\Omega_{2}^{2}+W^{2}-c\left(\Omega^{2}-\frac{W^{2}}{4 \mathrm{H}^{2}}\right)=0
\end{array}\right.
$$

onde $c, C$ são constantes não nulas e $\mathrm{H} \neq 0$.

Contudo, resulta do Lema 5.3.2 o seguinte

Lema 5.5.1. Seja $\phi$ uma solução de

$$
\Delta \phi=C^{2} e^{-2 \phi}-\mathrm{H}^{2} e^{2 \phi}
$$

onde $C$ e $\mathrm{H}$ são constantes não nulas. Então, para cada constante $c \neq 0$, fixadas condições iniciais sobre $\Omega, \Omega_{1}, \Omega_{2}, W$ satisfazendo a última equação de (5.80), este sistema admite solução. 
Demonstração. Se $\phi$ é solução de (5.76), podemos associar a ela uma superfície $X$ de curvatura média constante $\mathrm{H} \neq 0$. Pelo Teorema de Bonnet, existe uma superfície $\tilde{X}$, paralela a $X$, de curvatura gaussiana $\tilde{K}=4 \mathrm{H}^{2}$, cujas formas quadráticas são

$$
\begin{gathered}
\tilde{\mathrm{I}}=\left(\tilde{\omega}_{1}\right)^{2}+\left(\tilde{\omega}_{2}\right)^{2}=\frac{1}{4 \mathrm{H}^{2}}\left[\left(\mathrm{H} e^{\phi}-C e^{-\phi}\right)^{2} d u^{2}+\left(\mathrm{H} e^{\phi}+C e^{-\phi}\right)^{2} d v^{2}\right], \\
\tilde{\mathrm{II}}=\tilde{\omega}_{1} \tilde{\omega}_{13}+\tilde{\omega}_{2} \tilde{\omega}_{23}=\frac{1}{2 \mathrm{H}}\left(\mathrm{H} e^{\phi}-C e^{-\phi}\right)\left(\mathrm{H} e^{\phi}+C e^{-\phi}\right)\left(d u^{2}+d v^{2}\right) .
\end{gathered}
$$

Portanto, do Lema 5.3.2, para cada constante $c \neq 0$, fixadas condições iniciais sobre $\Omega, \Omega_{1}, \Omega_{2}, W$ satisfazendo a última equação de (5.41), este sistema admite solução. Como (5.80) é equivalente a (5.41), segue o resultado.

Como conseqüência deste lema, segue também do Corolário 5.3.4 o próximo resultado.

Teorema 5.5.2. Seja $\phi$ uma solução de (5.76), onde $C$ e $\mathrm{H}$ são constantes não nulas. Então, fixada $c \neq 0$, para cada solução não nula $\Omega, \Omega_{1}, \Omega_{2}, W$ do sistema (5.80), obtemos uma nova solução $\bar{\phi}$ de (5.76) definida por

$$
e^{\bar{\phi}}=\frac{|c|}{c} \frac{2 \mathrm{H} \Omega-W}{2 \mathrm{H} \Omega+W} e^{\phi} .
$$

Demonstração. Se $\phi$ é uma solução não nula de (5.76), decorre da Proposição 2.1.1 que existe uma superfície $X(u, v)$ de curvatura média constante $\mathrm{H} \neq 0$ associada a $\phi$ cujas formas quadráticas são:

$$
\begin{aligned}
\mathrm{I} & =e^{2 \phi}\left(d u^{2}+d v^{2}\right), \\
\mathrm{II} & =\left(\mathrm{H} e^{2 \phi}+C\right) d u^{2}+\left(\mathrm{H} e^{2 \phi}-C\right) d v^{2}
\end{aligned}
$$

e com 1-formas diferenciais dadas por (5.77). Considere a superfície $\tilde{X}$, paralela a $X$, de curvatura gaussiana $\tilde{K}=4 \mathrm{H}^{2}$ dada por (5.78) cujas 1-formas diferenciais são (5.79).

Se $\Omega, \Omega_{1}, \Omega_{2}, W$ é uma solução não nula de (5.80), do Corolário 5.3.4, temos que existe uma superfície $\tilde{X}^{s}$ associada a $\tilde{X}$, de mesma curvatura gaussiana $\tilde{K}^{s}=\tilde{K}=4 \mathrm{H}^{2}$, com 1 -formas definidas como na Observação 5.3.5:

$$
\begin{aligned}
\tilde{\omega}_{1}^{s} & =\frac{1}{4 \mathrm{H}^{2} \Omega^{2}-W^{2}}\left[\left(4 \mathrm{H}^{2} \Omega^{2}+W^{2}\right) \tilde{\omega}_{1}-2 \Omega W \tilde{\omega}_{13}\right], \\
\tilde{\omega}_{2}^{s} & =\frac{1}{4 \mathrm{H}^{2} \Omega^{2}-W^{2}}\left[\left(4 \mathrm{H}^{2} \Omega^{2}+W^{2}\right) \tilde{\omega}_{2}-2 \Omega W \tilde{\omega}_{23}\right], \\
\tilde{\omega}_{13}^{s} & =-\frac{1}{4 \mathrm{H}^{2} \Omega^{2}-W^{2}}\left[8 \mathrm{H}^{2} \Omega W \tilde{\omega}_{1}-\left(4 \mathrm{H}^{2} \Omega^{2}+W^{2}\right) \tilde{\omega}_{13}\right], \\
\tilde{\omega}_{23}^{s} & =-\frac{1}{4 \mathrm{H}^{2} \Omega^{2}-W^{2}}\left[8 \mathrm{H}^{2} \Omega W \tilde{\omega}_{2}-\left(4 \mathrm{H}^{2} \Omega^{2}+W^{2}\right) \tilde{\omega}_{23}\right] .
\end{aligned}
$$

Seja $X^{s}$ a superfície paralela a $\tilde{X}^{s}$ de curvatura média constante igual a $\mathrm{H}^{s}=\mathrm{H}$ dada por

$$
X^{s}=\tilde{X}^{s}-\frac{1}{2 \mathrm{H}} \tilde{e}_{3}^{s}
$$


Da prova da Proposição 3.1.7, as 1-formas associadas à superfície $X^{s}$ são

$$
\begin{aligned}
\omega_{1}^{s} & =\tilde{\omega}_{1}^{s}+\frac{1}{2 \mathrm{H}} \tilde{\omega}_{13}^{s}=A\left(\tilde{\omega}_{1}+\frac{1}{2 \mathrm{H}} \tilde{\omega}_{13}\right)=A e^{\phi} d u \\
\omega_{2}^{s} & =\tilde{\omega}_{2}^{s}+\frac{1}{2 \mathrm{H}} \tilde{\omega}_{23}^{s}=A\left(\tilde{\omega}_{2}+\frac{1}{2 \mathrm{H}} \tilde{\omega}_{23}\right)=A e^{\phi} d v \\
\omega_{13}^{s} & =\tilde{\omega}_{13}^{s}=\left(A \mathrm{H} e^{\phi}+\frac{1}{A} C e^{-\phi}\right) d u, \\
\omega_{23}^{s} & =\tilde{\omega}_{23}^{s}=\left(A \mathrm{H} e^{\phi}-\frac{1}{A} C e^{-\phi}\right) d v,
\end{aligned}
$$

onde $A:=\frac{2 \mathrm{H} \Omega-W}{2 \mathrm{H} \Omega+W}$. Note que, por (5.80),

$$
4 \mathrm{H}^{2} \Omega^{2}-W^{2}=\frac{4 \mathrm{H}^{2}}{c}\left(\Omega_{1}^{2}+\Omega_{2}^{2}+W^{2}\right)>0
$$

se, e somente se, $c>0$. Assim, definindo a função $\bar{\phi}$ por

$$
e^{\bar{\phi}}=\frac{|c|}{c} \frac{2 \mathrm{H} \Omega-W}{2 \mathrm{H} \Omega+W} e^{\phi},
$$

segue que as formas quadráticas de $X^{s}$ são

$$
\begin{aligned}
\mathrm{I}^{s} & =\left(\omega_{1}^{s}\right)^{2}+\left(\omega_{2}^{s}\right)^{2}=e^{2 \bar{\phi}}\left(d u^{2}+d v^{2}\right), \\
\mathrm{II}^{s} & =\omega_{1}^{s} \omega_{13}^{s}+\omega_{2}^{s} \omega_{23}^{s}=\left(\mathrm{H} e^{2 \bar{\phi}}+C\right) d u^{2}+\left(\mathrm{H} e^{2 \bar{\phi}}-C\right) d v^{2} .
\end{aligned}
$$

Logo, pela Proposição 2.1.1, $\bar{\phi}$ é solução de (5.76). 


\section{Apêndice A}

\section{Teoria local das superfícies}

Vamos apresentar aqui algumas noções básicas da teoria local das superfícies, com o objetivo de facilitar o entendimento do leitor durante o estudo da teoria das transformações de superfícies de curvatura constante proposto nesta dissertação.

\section{A.1 Superfície parametrizada regular}

Definição A.1.1. Uma aplicação $X: U \subset \mathbb{R}^{2} \rightarrow \mathbb{R}^{3}$, onde $U$ é um conjunto aberto, é dita uma superfície parametrizada se $X$ for diferenciável de classe $C^{\infty}$. O conjunto $X(U) \subset \mathbb{R}^{3}$ é chamado de traço de $X$. Além disso, $X$ é dita superfície parametrizada regular se, para todo $q \in U$, a diferencial $d X_{q}: \mathbb{R}^{2} \rightarrow \mathbb{R}^{3}$ for injetiva (isto é, os vetores $X_{u}, X_{v}$ são linearmente independentes para todo $q \in U)$.

As variáveis $u, v$ são chamadas de parâmetros de $X$ e, fixado $q=\left(u_{0}, v_{0}\right) \in U$, as aplicações $\alpha(u)=X\left(u, v_{0}\right)$ e $\beta(v)=X\left(u_{0}, v\right)$ são ditas curvas coordenadas u-paramétrica e $v$-paramétrica, respectivamente.

Dada uma superfície parametrizada regular, a partir dela podemos obter várias outras superfícies de mesmo traço da seguinte forma:

Proposição A.1.2. Seja $X: U \subset \mathbb{R}^{2} \rightarrow \mathbb{R}^{3}$ uma superfície parametrizada regular. Se uma aplicação $h: \bar{U} \subset \mathbb{R}^{2} \rightarrow U$ for diferenciável, sobrejetiva e com jacobiano não nulo, então

$$
\bar{X}=X \circ h: \bar{U} \longrightarrow \mathbb{R}^{3}
$$

é uma superfície parametrizada regular cujo traço é igual ao de $X$, isto é, $\bar{X}(\bar{U})=X(U)$.

A aplicação $\bar{X}$ é denominada de reparametrização de $X$ por $h$ e a função $h$ é dita uma mudança de parâmetros.

Definição A.1.3. Sejam $X: U \subset \mathbb{R}^{2} \rightarrow \mathbb{R}^{3}$ uma superfície parametrizada regular de traço $S=X(U)$ e $q \in U$. Chamamos de plano tangente à superfície $X$ em $q$ o espaço vetorial bidimensional $d X_{q}\left(\mathbb{R}^{2}\right) \subset \mathbb{R}^{3}$, que será denotado por $\mathrm{T}_{X(q)} S$ e cujos elementos são ditos vetores tangentes a $X$ no ponto $q$.

Note que se $w \in \mathrm{T}_{X(q)} S$, então existe $v \in \mathbb{R}^{2}$, escrito na forma $v=a \frac{\partial}{\partial u}+b \frac{\partial}{\partial v}, a, b \in \mathbb{R}$, tal que $w=d X_{q}(v)$, isto é, como $d X_{q}$ é uma aplicação linear,

$$
w=d X_{q}\left(a \frac{\partial}{\partial u}+b \frac{\partial}{\partial v}\right)=a X_{u}(q)+b X_{v}(q) .
$$


Portanto o espaço tangente $\mathrm{T}_{X(q)} S$ é gerado pelos vetores $X_{u}(q)$ e $X_{v}(q)$.

Definição A.1.4. Um vetor de $\mathbb{R}^{3}$ é dito normal à superfície $X: U \subset \mathbb{R}^{2} \rightarrow \mathbb{R}^{3}$ no ponto $q \in U$ se for ortogonal ao plano $\mathrm{T}_{X(q)} S$, isto é, ortogonal a todos os vetores tangentes a $X$ em $q$.

Como o vetor $X_{u} \times X_{v}$ é normal à superfície $X$, podemos definir a aplicação

$$
\begin{aligned}
N: U & \longrightarrow \mathbb{S}^{2}(1) \subset \mathbb{R}^{3} \\
q & \longmapsto N(q)=\frac{X_{u} \wedge X_{v}}{\left\|X_{u} \wedge X_{v}\right\|}(q),
\end{aligned}
$$

onde $\mathbb{S}^{2}(1)$ é a esfera unitária. Esta aplicação é chamada aplicação normal de Gauss.

\section{A.2 Primeira e segunda formas quadráticas}

No desenvolvimento da teoria local das superfícies utilizaremos duas formas quadráticas. A primeira está relacionada essencialmente com as propriedades métricas da superfície, enquanto a segunda, com o estudo das curvaturas de curvas da superfície. Também estas formas determinam localmente uma superfície, como veremos no Teorema Fundamental das Superfícies.

\section{Primeira forma quadrática}

O produto escalar de $\mathbb{R}^{3},\langle$,$\rangle , induz em cada plano tangente de uma superfície parametrizada$ regular $X$ um produto interno:

Definição A.2.1. Sejam $X: U \subset \mathbb{R}^{2} \rightarrow S \subset \mathbb{R}^{3}$ uma superfície parametrizada regular e $q \in U$. A aplicação

$$
\begin{aligned}
\mathrm{I}_{q}: \mathrm{T}_{X(q)} S \times \mathrm{T}_{X(q)} S & \longrightarrow \mathbb{R} \\
\left(w_{1}, w_{2}\right) & \longmapsto \mathrm{I}_{q}\left(w_{1}, w_{2}\right)=\left\langle w_{1}, w_{2}\right\rangle
\end{aligned}
$$

é denominada primeira forma quadrática de $X$ em $q$. Denotaremos por $\mathrm{I}_{q}(w)=\mathrm{I}_{q}(w, w)=\|w\|^{2}$.

Supondo $q=\left(u_{0}, v_{0}\right) \in U$ e $w=a X_{u}+b X_{v} \in \mathrm{T}_{X(q)} S$, onde $a, b \in \mathbb{R}$, então

$$
\mathrm{I}_{q}(w)=a^{2}\left\langle X_{u}, X_{u}\right\rangle\left(u_{0}, v_{0}\right)+2 a b\left\langle X_{u}, X_{v}\right\rangle\left(u_{0}, v_{0}\right)+b^{2}\left\langle X_{v}, X_{v}\right\rangle\left(u_{0}, v_{0}\right) .
$$

Usando a notação

$$
\begin{aligned}
& E\left(u_{0}, v_{0}\right)=\left\langle X_{u}, X_{u}\right\rangle\left(u_{0}, v_{0}\right), \\
& F\left(u_{0}, v_{0}\right)=\left\langle X_{u}, X_{v}\right\rangle\left(u_{0}, v_{0}\right), \\
& G\left(u_{0}, v_{0}\right)=\left\langle X_{v}, X_{v}\right\rangle\left(u_{0}, v_{0}\right),
\end{aligned}
$$

reescrevemos (A.1) como:

$$
\mathrm{I}_{q}(w)=a^{2} E\left(u_{0}, v_{0}\right)+2 a b F\left(u_{0}, v_{0}\right)+b^{2} G\left(u_{0}, v_{0}\right) .
$$

Variando $(u, v) \in U$, obtemos funções diferenciáveis $E, F, G: U \rightarrow \mathbb{R}$, chamadas coeficientes da primeira forma quadrática que satisfazem as seguintes propriedades: 
- $E(u, v)>0$ e $G(u, v)>0$;

- $E(u, v) G(u, v)-F^{2}(u, v)>0$,

para todo $(u, v) \in U$.

Considerando, agora, $X(u, v)$ e $\bar{X}(u, v),(u, v) \in U$, duas superfícies parametrizadas regulares com coeficientes da primeira forma quadrática dados, respectivamente, por $E, F, G$ e $\bar{E}, \bar{F}, \bar{G}$, temos a seguinte:

Definição A.2.2. As superfícies $X$ e $\bar{X}$ são ditas isométricas se, e somente se, $\mathrm{I}_{q}=\overline{\mathrm{I}}_{q}$, para todo $q \in U$, isto é, $E=\bar{E}, F=\bar{F}, G=\bar{G}$.

\section{Segunda forma quadrática}

Definição A.2.3. Sejam $X: U \subset \mathbb{R}^{2} \rightarrow \mathbb{R}^{3}$ uma superfície parametrizada regular, $N: U \rightarrow \mathbb{R}^{3}$ a aplicação normal de Gauss e $q \in U$. Definimos a segunda forma quadrática de $X$ em $q$ como sendo a aplicação bilinear e simétrica dada por:

$$
\begin{aligned}
\mathrm{II}_{q}: \mathrm{T}_{X(q)} S \times \mathrm{T}_{X(q)} S & \longrightarrow \mathbb{R} \\
\left(w_{1}, w_{2}\right) & \longmapsto \mathrm{II}_{q}\left(w_{1}, w_{2}\right)=-\left\langle d X_{q}\left(v_{1}\right), d N_{q}\left(v_{2}\right)\right\rangle,
\end{aligned}
$$

onde $w_{i}=d X_{q}\left(v_{i}\right)$. Novamente, denotaremos por $\operatorname{II}_{q}(w)=\operatorname{II}_{q}(w, w)$, para todo $w \in \mathrm{T}_{X(q)} S$.

Como $d X_{q}\left(\mathbb{R}^{2}\right)=\mathrm{T}_{X(q)} S$ podemos olhar a segunda forma quadrática em $q$ como a função

$$
\begin{aligned}
\mathrm{II}_{q}: \mathbb{R}^{2} \times \mathbb{R}^{2} & \longrightarrow \mathbb{R} \\
\left(v_{1}, v_{2}\right) & \longmapsto \mathrm{II}_{q}\left(v_{1}, v_{2}\right)=-\left\langle d X_{q}\left(v_{1}\right), d N_{q}\left(v_{2}\right)\right\rangle .
\end{aligned}
$$

Supondo $q=\left(u_{0}, v_{0}\right) \in U$ e $v=a \frac{\partial}{\partial u}+b \frac{\partial}{\partial v} \in \mathbb{R}^{2}$, onde $a, b \in \mathbb{R}$, temos que

$$
\mathrm{II}_{q}(v)=-a^{2}\left\langle X_{u}, N_{u}\right\rangle\left(u_{0}, v_{0}\right)-a b\left[\left\langle X_{u}, N_{v}\right\rangle\left(u_{0}, v_{0}\right)+\left\langle X_{v}, N_{u}\right\rangle\left(u_{0}, v_{0}\right)\right]-b^{2}\left\langle X_{v}, N_{v}\right\rangle\left(u_{0}, v_{0}\right) .
$$

Como $\left\langle X_{u}, N\right\rangle=0=\left\langle X_{v}, N\right\rangle$, derivando estas relações, obtemos:

$$
\begin{aligned}
& \left\langle X_{u v}, N\right\rangle+\left\langle X_{u}, N_{v}\right\rangle=0, \\
& \left\langle X_{v u}, N\right\rangle+\left\langle X_{v}, N_{u}\right\rangle=0,
\end{aligned}
$$

de onde concluímos que $\left\langle X_{u}, N_{v}\right\rangle=\left\langle X_{v}, N_{u}\right\rangle$. Portanto

$$
\mathrm{II}_{q}(v)=-a^{2}\left\langle X_{u}, N_{u}\right\rangle\left(u_{0}, v_{0}\right)-2 a b\left\langle X_{u}, N_{v}\right\rangle\left(u_{0}, v_{0}\right)-b^{2}\left\langle X_{v}, N_{v}\right\rangle\left(u_{0}, v_{0}\right)
$$

e, usando a notação

$$
\begin{aligned}
& e\left(u_{0}, v_{0}\right)=-\left\langle X_{u}, N_{u}\right\rangle\left(u_{0}, v_{0}\right), \\
& f\left(u_{0}, v_{0}\right)=-\left\langle X_{u}, N_{v}\right\rangle\left(u_{0}, v_{0}\right), \\
& g\left(u_{0}, v_{0}\right)=-\left\langle X_{v}, N_{v}\right\rangle\left(u_{0}, v_{0}\right),
\end{aligned}
$$

podemos reescrever a equação (A.2) como

$$
\operatorname{II}_{q}(v)=a^{2} e\left(u_{0}, v_{0}\right)+2 a b f\left(u_{0}, v_{0}\right)+b^{2} g\left(u_{0}, v_{0}\right) .
$$

Variando $(u, v)$ em $U$, temos as funções diferenciáveis $e, f, g: U \rightarrow \mathbb{R}$, denominadas coeficientes da segunda forma quadrática. 
Definição A.2.4. Dados $X: U \subset \mathbb{R}^{2} \rightarrow \mathbb{R}^{3}$ uma superfície parametrizada regular e $q \in U$, a aplicação diferenciável $\kappa_{n}: \mathrm{T}_{X(q)} S-\{0\} \rightarrow \mathbb{R}$ dada por:

$$
\kappa_{n}(w)=\frac{\mathrm{II}_{q}(w)}{\mathrm{I}_{q}(w)}
$$

é denominada função curvatura normal de $X$ em $q$.

Observe que, para todo $\lambda \neq 0$, temos $\kappa_{n}(\lambda w)=\kappa_{n}(w)$.

\section{A.3 Curvatura média e curvatura gaussiana}

A aplicação curvatura normal $\kappa_{n}$ admite um valor máximo e um mínimo. Estes valores são chamados de curvaturas principais e a existência deles será provada no próximo resultado. Para isso, dados $X(u, v),(u, v) \in U$, uma superfície parametrizada regular e $q=\left(u_{0}, v_{0}\right) \in U$, denotaremos os coeficientes das formas quadráticas de $X$ em $q$ por $E_{0}, F_{0}, G_{0}$ e $e_{0}, f_{0}, g_{0}$.

Proposição A.3.1. Sejam $X: U \subset \mathbb{R}^{2} \rightarrow \mathbb{R}^{3}$ uma superfície parametrizada regular, $S=X(U)$ e $\kappa_{n}: \mathrm{T}_{X(q)} S-\{0\} \rightarrow \mathbb{R}$ a função curvatura normal de $X$ em $q=\left(u_{0}, v_{0}\right) \in U$. Então existem dois vetores ortonormais $e_{1}, e_{2} \in \mathrm{T}_{X(q)} S$ tais que as curvaturas normais $\kappa_{1}$ e $\kappa_{2}$ nestas direções são os valores mínimo e máximo da função $\kappa_{n}$.

Demonstração. Note que se $\kappa_{n}$ for constante, então quaisquer dois vetores ortonormais satisfazem as condições da proposição. Suponha, assim, que $\kappa_{n}$ não é constante e considere a função diferenciável dada por

$$
\begin{aligned}
\tilde{\kappa}_{n}: \mathbb{R}^{2}-\{(0,0)\} & \longrightarrow \mathbb{R} \\
(a, b) & \longmapsto \tilde{\kappa}_{n}(a, b)=\kappa_{n}\left(a X_{u}(q)+b X_{v}(q)\right)
\end{aligned}
$$

ou seja,

$$
\tilde{\kappa}_{n}(a, b)=\frac{a^{2} e_{0}+2 a b f_{0}+b^{2} g_{0}}{a^{2} E_{0}+2 a b F_{0}+b^{2} G_{0}} .
$$

Note que, para todo $\lambda \neq 0$, temos $\tilde{\kappa}_{n}(\lambda a, \lambda b)=\tilde{\kappa}_{n}(a, b)$. Portanto, para obter os valores mínimo e máximo de $\tilde{\kappa}_{n}$ basta restringir esta função à circunferência $C$ de $\mathbb{R}^{2}$ dada por $a^{2}+b^{2}=1$. Como $\tilde{\kappa}_{n}$ é uma função contínua, existem dois pontos $\left(a_{1}, b_{1}\right)$ e $\left(a_{2}, b_{2}\right)$ de $C$ tais que

$$
\kappa_{1}=\tilde{\kappa}_{n}\left(a_{1}, b_{1}\right) \quad \text { e } \quad \kappa_{2}=\tilde{\kappa}_{n}\left(a_{2}, b_{2}\right)
$$

são respectivamente o mínimo e o máximo de $\tilde{\kappa}_{n}$ em $C$, isto é,

$$
\kappa_{1} \leqslant \tilde{\kappa}_{n}(a, b) \leqslant \kappa_{2}, \quad \forall(a, b) \in \mathbb{R}^{2}-\{(0,0)\}
$$

Como $\kappa_{n}$ não é constante, segue que $\tilde{\kappa}_{n}$ também não é constante e, portanto, $\kappa_{1}<\kappa_{2}$.

Se $w_{i}=a_{i} X_{u}(q)+b_{i} X_{v}(q), i=1,2$, temos que

$$
\kappa_{1}=\kappa_{n}\left(w_{1}\right) \leqslant \kappa_{n}(w) \leqslant \kappa_{n}\left(w_{2}\right)=\kappa_{2}, \quad \forall w \in \mathrm{T}_{X(q)} S-\{0\} .
$$


Vamos mostrar que $w_{1}$ e $w_{2}$ são ortogonais. Para isso, como $\left(a_{1}, b_{1}\right)$ e $\left(a_{2}, b_{2}\right)$ fornecem o mínimo e o máximo da função $\tilde{\kappa}_{n}$, então as derivadas parciais desta função, nestes pontos, são nulas. Assim,

$$
\begin{aligned}
& \left(e_{0}-\kappa_{1} E_{0}\right) a_{1}+\left(f_{0}-\kappa_{1} F_{0}\right) b_{1}=0 \\
& \left(f_{0}-\kappa_{1} F_{0}\right) a_{1}+\left(g_{0}-\kappa_{1} G_{0}\right) b_{1}=0 \\
& \left(e_{0}-\kappa_{2} E_{0}\right) a_{2}+\left(f_{0}-\kappa_{2} F_{0}\right) b_{2}=0 \\
& \left(f_{0}-\kappa_{2} F_{0}\right) a_{2}+\left(g_{0}-\kappa_{2} G_{0}\right) b_{2}=0 .
\end{aligned}
$$

Subtraindo a equação (A.4), multiplicada por $a_{2}$, da equação (A.6), multiplicada por $a_{1}$, obtemos

$$
\left(\kappa_{1}-\kappa_{2}\right) E_{0} a_{1} a_{2}+f_{0}\left(a_{1} b_{2}-a_{2} b_{1}\right)+F_{0}\left(\kappa_{1} a_{2} b_{1}-\kappa_{2} a_{1} b_{2}\right)=0 .
$$

Analogamente, subtraindo a equação (A.5), multiplicada por $b_{2}$, da equação (A.7), multiplicada por $b_{1}$, obtemos

$$
-f_{0}\left(a_{1} b_{2}-a_{2} b_{1}\right)+F_{0}\left(\kappa_{1} a_{1} b_{2}-\kappa_{2} a_{2} b_{1}\right)+\left(\kappa_{1}-\kappa_{2}\right) G_{0} b_{1} b_{2}=0 .
$$

Somando as últimas duas relações obtidas, encontramos

$$
\left(\kappa_{1}-\kappa_{2}\right)\left(a_{1} a_{2} E_{0}+a_{1} b_{2} F_{0}+a_{2} b_{1} F_{0}+b_{1} b_{2} G_{0}\right)=0 .
$$

Portanto, com $\kappa_{1} \neq \kappa_{2}$, concluímos que

$$
\left\langle w_{1}, w_{2}\right\rangle=a_{1} a_{2} E_{0}+a_{1} b_{2} F_{0}+a_{2} b_{1} F_{0}+b_{1} b_{2} G_{0}=0 .
$$

Assim, obtemos dois vetores ortogonais $w_{1}$ e $w_{2}$ que dão o mínimo e o máximo, respectivamente, da função $\kappa_{n}$. Mas $w_{1}$ e $w_{2}$ não necessariamente são unitários. Portanto considerando

$$
e_{1}=\frac{w_{1}}{\left\|w_{1}\right\|} \quad \text { e } \quad e_{2}=\frac{w_{2}}{\left\|w_{2}\right\|},
$$

e como $\kappa_{n}(\lambda w)=\kappa_{n}(w)$, concluímos que $e_{1}$ e $e_{2}$ satisfazem as condições da proposição.

As curvaturas $\kappa_{1}$ e $\kappa_{2}$, que definimos na proposição anterior, são as curvaturas principais da superfície $S$ no ponto $q$. Os vetores $e_{1}$ e $e_{2}$ chamamos de vetores principais em $q$ e as direções determinadas por estes vetores de direções principais neste ponto.

Note que estes vetores principais determinam a curvatura normal em qualquer direção. Mais precisamente,

Proposição A.3.2 (Fórmula de Euler). Seja $X: U \subset \mathbb{R}^{2} \rightarrow \mathbb{R}^{3}$ uma superfície parametrizada regular com curvaturas principais $\kappa_{1}, \kappa_{2}$ e vetores principais $e_{1}, e_{2}$ em $q=\left(u_{0}, v_{0}\right) \in U$. Então, para todo vetor unitário $w \in \mathrm{T}_{X(q)} S$, escrito como

$$
w=\cos \theta e_{1}+\operatorname{sen} \theta e_{2},
$$

temos que

$$
\kappa_{n}(w)=\kappa_{1} \cos ^{2} \theta+\kappa_{2} \operatorname{sen}^{2} \theta .
$$

Demonstração. Para a prova veja [22], página 169. 
Definição A.3.3. Seja $X: U \subset \mathbb{R}^{2} \rightarrow \mathbb{R}^{3}$ uma superfície parametrizada regular. Definimos a curvatura média de $X$ no ponto $q \in U$ como sendo a semi-soma das curvaturas principais de $X$ em $q$, isto é,

$$
\mathrm{H}(q)=\frac{\kappa_{1}+\kappa_{2}}{2}(q)
$$

e a curvatura gaussiana de $X$ em $q$ pelo produto:

$$
K(q)=\kappa_{1}(q) \kappa_{2}(q) .
$$

Segue desta definição que as curvaturas principais em $q$ são soluções da equação

$$
\kappa^{2}-2 \mathrm{H}(q) \kappa+K(q)=0, \quad \text { isto é, } \quad \kappa_{i}=\mathrm{H} \pm \sqrt{\mathrm{H}^{2}-K}, \quad i=1,2 .
$$

Observe que uma mudança de parâmetros pode alterar o sinal das curvaturas principais e, portanto, da curvatura média, no entanto a curvatura gaussiana continua a mesma.

Usando o fato de que as curvaturas principais $\kappa_{1}$ e $\kappa_{2}$ são o mínimo e o máximo da função curvatura normal, podemos obter as curvaturas $\mathrm{H}$ e $K$ a partir dos coeficientes das formas quadráticas.

Proposição A.3.4. Seja $X: U \subset \mathbb{R}^{2} \rightarrow \mathbb{R}^{3}$ uma superfície parametrizada regular e $q \in U$. Então

$$
\mathrm{H}(q)=\frac{e G-2 f F+E g}{2\left(E G-F^{2}\right)}(q), \quad K(q)=\frac{e g-f^{2}}{E G-F^{2}}(q) .
$$

Demonstração. A prova pode ser encontrada em [22], página 171.

Esta proposição permite-nos calcular as curvaturas média e gaussiana de uma superfície sem precisarmos nos preocupar em encontrar as curvaturas principais.

Exemplo A.3.5. Considere a pseudo-esfera parametrizada por:

$$
X(u, v)=(\operatorname{sech} u \cos v, \operatorname{sech} u \operatorname{sen} v, u-\tanh u),
$$

sendo $u>0$ (veja Figura 3.4). Calculando os coeficientes das formas quadráticas de $X$, temos:

$$
\begin{aligned}
E=\tanh ^{2} u, & F=0, \quad G=\operatorname{sech}^{2} u, \\
e=-\operatorname{sech} u \tanh u, & f=0, \quad g=\operatorname{sech} u \tanh u,
\end{aligned}
$$

de onde resulta que

$$
\mathrm{H}(u)=\frac{\cosh u}{\tanh u} \quad \text { e } \quad K \equiv-1 .
$$

Por outro lado, a partir dos coeficientes da primeira e segunda formas quadráticas, facilmente encontramos as curvaturas principais e os vetores principais através do resultado a seguir.

Proposição A.3.6. Seja $X: U \subset \mathbb{R}^{2} \rightarrow \mathbb{R}^{3}$ uma superfície parametrizada regular.

(i) Um número real $\kappa$ é uma curvatura principal de $X$ no ponto $q \in U$ se, e só se,

$$
\left|\begin{array}{cc}
e-\kappa E & f-\kappa F \\
f-\kappa F & g-\kappa G
\end{array}\right|(q)=0 .
$$


(ii) Um vetor $w=a X_{u}(q)+b X_{v}(q) \in \mathrm{T}_{X(q)} S$ é uma direção principal de $X$ em $q$ se, e somente se,

$$
b^{2}(F g-f G)(q)+a b(E g-e G)(q)+a^{2}(E f-e F)(q)=0 .
$$

Demonstração. A prova desta proposição pode ser encontrada em [22], Capítulo 3.

Se $X(u, v),(u, v) \in U$, for uma superfície parametrizada regular cujas curvas coordenadas são ortogonais, ou seja, $F=0$, a curvatura gaussiana de $X$ pode ser calculada pela equação de Gauss:

$$
K=-\frac{1}{\sqrt{E G}}\left[\left(\frac{(\sqrt{E})_{v}}{\sqrt{G}}\right)_{v}+\left(\frac{(\sqrt{G})_{u}}{\sqrt{E}}\right)_{u}\right] .
$$

Também, neste caso, as equações de Codazzi-Mainardi são dadas por:

$$
\begin{aligned}
& 2 E G\left(e_{v}-f_{u}\right)-(E g+G e) E_{v}-f\left(E G_{u}-G E_{u}\right)=0, \\
& 2 E G\left(f_{v}-g_{u}\right)+(E g+G e) G_{u}-f\left(E G_{v}-G E_{v}\right)=0 .
\end{aligned}
$$

A importância das equações de Gauss e de Codazzi-Mainardi está no seguinte resultado.

Teorema A.3.7 (Teorema Fundamental das Superfícies). Seja U um subconjunto aberto de $\mathbb{R}^{2}$ simplesmente conexo e suponha que, para cada $q \in U$, as formas quadráticas $\mathrm{I}_{q}$ e $\mathrm{II}_{q}$ possuem como coeficientes as funções diferenciáveis $E, F, G$ e e, $f, g$, respectivamente. Se $\mathrm{I}_{q}$ é definida positiva e as equações de Gauss e de Codazzi-Mainardi estão satisfeitas, então, a menos de movimento rígido de $\mathbb{R}^{3}$, existe uma única superfície $X: U \subset \mathbb{R}^{2} \rightarrow \mathbb{R}^{3}$ tal que, para todo $q \in U, \mathrm{I}_{q}$ e $\mathrm{II}_{q}$ são a primeira e segunda formas quadráticas de $X$ em q, respectivamente.

Demonstração. A prova encontra-se em [13], página 375.

\section{A.3.1 Classificação dos pontos de uma superfície}

O sinal da curvatura gaussiana em um ponto $q \in U$ permite-nos estudar localmente o comportamento de uma superfície $X: U \subset \mathbb{R}^{2} \rightarrow \mathbb{R}^{3}$. Assim, denotando por $K$ e $\mathrm{H}$ as respectivas curvaturas gaussiana e média de $X$, chamamos o ponto $q$ de:

- elíptico, se $K(q)>0$

- hiperbólico, se $K(q)<0$;

- parabólico, se $K(q)=0$ e $\mathrm{H}(q) \neq 0$;

- planar, se $K(q)=0$ e $\mathrm{H}(q)=0$.

Exemplo A.3.8. Considere o toro parametrizado por

$$
X(u, v)=((a+r \cos u) \cos v,(a+r \cos u) \operatorname{sen} v, r \operatorname{sen} u), \quad a>0,0<r<a,
$$

Calculando os coeficientes de suas formas quadráticas, temos

$$
\begin{array}{ll}
E=r^{2}, & F=0, \quad G=(a+r \cos u)^{2}, \\
e=-r, & f=0, \quad g=-(a+r \cos u) \cos u,
\end{array}
$$


de onde resulta que

$$
K(u)=\frac{\cos u}{r(a+r \cos u)} \quad \text { e } \quad \mathrm{H}(u)=-\frac{a+2 r \cos u}{2 r(a+r \cos u)} .
$$

Portanto, vemos que:

- quando $-\frac{\pi}{2}<u<\frac{\pi}{2},(u, v)$ é um ponto elíptico;

- quando $\frac{\pi}{2}<u<\frac{3 \pi}{2},(u, v)$ é um ponto hiperbólico;

- quando $u= \pm \frac{\pi}{2},(u, v)$ é um ponto parabólico.

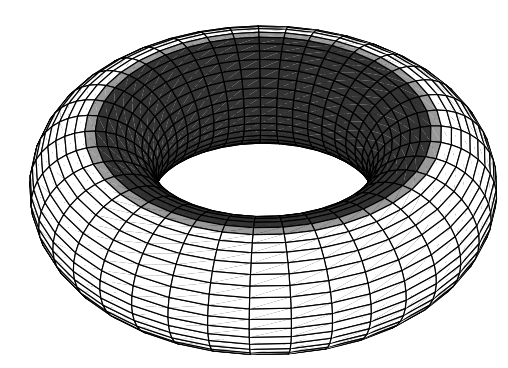

Figura A.1: Pontos hiperbólicos (região mais escura), pontos elípticos (região branca) e pontos parabólicos (região cinza).

Definição A.3.9. Dada $X: U \subset \mathbb{R}^{2} \rightarrow \mathbb{R}^{3}$ uma superfície parametrizada regular, dizemos que um ponto $q \in U$ é umbílico se as curvaturas principais nele coincidem, isto é, $\kappa_{1}(q)=\kappa_{2}(q)$.

Como, pela Definição A.3.3,

$$
\mathrm{H}^{2}(q)-K(q)=\frac{\left(\kappa_{1}-\kappa_{2}\right)^{2}}{4}(q),
$$

segue que $q$ é um ponto umbílico de $X$ se, e somente se, $\mathrm{H}^{2}(q)-K(q)=0$.

\section{A.4 Superfícies de Weingarten}

Considerando $X: U \subset \mathbb{R}^{2} \rightarrow \mathbb{R}^{3}$ uma superfície parametrizada regular, a partir das curvaturas principais $\kappa_{1}, \kappa_{2}$ de $X$, temos a seguinte:

Definição A.4.1. Dizemos que $X$ é uma superfície de Weingarten se existe uma relação (não trivial) $\phi$ entre suas curvaturas principais, isto é, $\phi\left(\kappa_{1}, \kappa_{2}\right)=0$.

Exemplo A.4.2. Considere a superfície parametrizada por:

$$
X(u, v)=\left(u^{2} \cos v, u^{2} \operatorname{sen} v, a u\right), \quad(u, v) \in \mathbb{R}^{2}, a \in \mathbb{R},
$$

obtida pela rotação da curva $\alpha(v)=\left(u^{2}, 0, a u\right)$ ao redor do eixo $z$. 


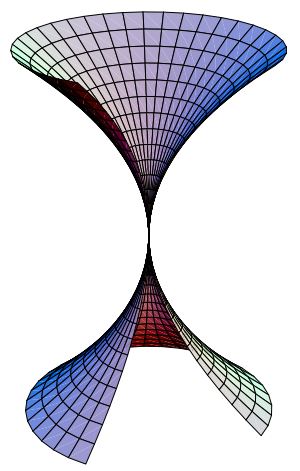

Figura A.2: Superfície obtida pela rotação da curva $\alpha(u)=\left(u^{2}, 0, a u\right)$ ao redor do eixo $z$.

Calculando os coeficientes da primeira e da segunda formas quadráticas, temos

$$
\begin{array}{cl}
E=a^{2}+4 u^{2}, & F=0, \quad G=u^{4}, \\
e=-\frac{2 a}{\left(a^{2}+4 u^{2}\right)^{\frac{1}{2}}}, & f=0, \quad g=\frac{a u^{2}}{\left(9+4 u^{2}\right)^{\frac{1}{2}}}
\end{array}
$$

de onde resulta

$$
K(u)=-\frac{2 a^{2}}{u^{2}\left(a^{2}+4 u^{2}\right)^{2}}, \quad \mathrm{H}(u)=\frac{a^{3}+2 a u^{2}}{2 u^{2}\left(a^{2}+4 u^{2}\right)^{\frac{3}{2}}} .
$$

Portanto, $X$ é uma superfície de Weingarten cujas curvaturas principais:

$$
\kappa_{1}=\frac{a}{u^{2}\left(a^{2}+4 u^{2}\right)^{\frac{1}{2}}}, \quad \kappa_{2}=-\frac{2 a}{\left(a^{2}+4 u^{2}\right)^{\frac{3}{2}}},
$$

satisfazem a seguinte relação:

$$
\frac{2 \kappa_{1}}{a^{2}+4 u^{2}}+\frac{\kappa_{2}}{u^{2}}=0
$$

Observe que a Definição A.4.1 é equivalente a dizer que existe uma relação (não trivial) entre as curvaturas média e gaussiana da superfície, $\psi(\mathrm{H}, K)=0$. Assim, vemos que as superfícies de curvatura média ou de curvatura gaussiana constante são exemplos de superfícies de Weingarten. Em particular,

Definição A.4.3. Dizemos que uma superfície parametrizada regular $X: U \subset \mathbb{R}^{2} \rightarrow \mathbb{R}^{3}$ é minima se possui curvatura média nula, isto é, $\mathrm{H}(q)=0$ para todo $q \in U$.

Exemplo A.4.4. Considere a superfície de Enneper (veja Figura 2.1) parametrizada por:

$$
X(u, v)=\left(u-\frac{u^{3}}{3}+u v^{2}, v-\frac{v^{3}}{3}+v u^{2}, u^{2}-v^{2}\right) .
$$

Esta é uma superfície mínima com coeficientes da primeira e segunda formas quadráticas dados por:

$$
\begin{aligned}
& E=\left(1+u^{2}+v^{2}\right)^{2}, F=0, \quad G=\left(1+u^{2}+v^{2}\right)^{2}, \\
& e=2, \quad f=0, \quad g=-2 .
\end{aligned}
$$




\section{A.5 Linha de curvatura; curva assintótica; direções conjugadas}

Sejam $X(u, v),(u, v) \in U$, uma superfície parametrizada regular e $u, v: I \subset \mathbb{R} \rightarrow \mathbb{R}$ funções diferenciáveis a um parâmetro $t \in I$. A curva parametrizada diferenciável $\alpha(t)=X(u(t), v(t))$ é dita uma curva regular da superfície $X$ se $\alpha^{\prime}(t) \neq 0$, para todo $t \in I$.

Apresentaremos, nesta seção, dois tipos de curvas regulares especiais: as linhas de curvatura e as curvas assintóticas.

Definição A.5.1. Dizemos que uma curva regular $\alpha(t)=X(u(t), v(t)), t \in I$, é uma linha de curvatura se, para todo $t \in I$, o vetor $\alpha^{\prime}(t)$ for uma direção principal de $X$ em $(u(t), v(t))$.

As linhas de curvatura originam uma classe importante de parametrizações.

Definição A.5.2. Uma parametrização $X: U \subset \mathbb{R}^{2} \rightarrow \mathbb{R}^{3}$ é dita principal se suas curvas coordenadas são linhas de curvatura.

Proposição A.5.3. Seja $X: U \subset \mathbb{R}^{2} \rightarrow \mathbb{R}^{3}$ uma superfície sem pontos umbilicos. Então, para todo $q_{0} \in U$, existe uma vizinhança $U_{0}$ de $q_{0}$ e uma mudança de parâmetros $h: V_{0} \subset \mathbb{R}^{2} \rightarrow U_{0}$ tal que $X \circ h$ é uma reparametrização principal.

Proposição A.5.4. Se $X(u, v)$ é uma superfície sem pontos umbílicos, então as curvas coordenadas são linhas de curvatura se, e somente se, $F=0=f$. Neste caso, as curvaturas principais de $X$ são dadas por

$$
\kappa_{1}=\frac{e}{E} \quad e \quad \kappa_{2}=\frac{g}{G}
$$

Demonstração. A prova deste resultado pode ser encontrado em [13], Capítulo 3.

Definição A.5.5. Sejam $X: U \subset \mathbb{R}^{2} \rightarrow S \subset \mathbb{R}^{3}$ uma superfície parametrizada regular e um ponto $q \in U$. Dizemos que um vetor não nulo $w \in \mathrm{T}_{X(q)} S$ é uma direção assintótica se a curvatura normal em $q$ na direção de $w$ for nula, ou seja,

$$
\mathrm{II}_{q}(w)=0 .
$$

Podemos determinar a quantidade de direções assintóticas existente em um ponto $q$ considerando sua classificação, ou seja,

Proposição A.5.6. Seja $X: U \subset \mathbb{R}^{2} \rightarrow \mathbb{R}^{3}$ uma superfície parametrizada regular e $q \in U$, temos que

(i) se q for um ponto elíptico, não existem direções assintóticas neste ponto;

(ii) se q for um ponto hiperbólico, existem exatamente duas direções assintóticas e as direções principais bissectam as direções assintóticas;

(iii) se q for um ponto parabólico, existe uma única direção assintótica, que também é principal;

(iv) se q for um ponto planar, qualquer direção é assintótica. 
Demonstração. Indicando por $\kappa_{1}, \kappa_{2}$ as curvaturas principais da superfície em $q$ e por $e_{1}, e_{2}$ os vetores principais neste ponto, segue da Proposição A.3.2 que a curvatura normal de um vetor unitário $w \in \mathrm{T}_{X(q)} S$, escrito como

$$
w=\cos \theta e_{1}+\operatorname{sen} \theta e_{2}
$$

é dada pela fórmula de Euler:

$$
\kappa_{n}(w)=\kappa_{1} \cos ^{2} \theta+\kappa_{2} \operatorname{sen}^{2} \theta
$$

Assim,

(i) se $K(q)>0$, então $\kappa_{1}$ e $\kappa_{2}$ têm o mesmo sinal e, portanto, $\kappa_{n}(w) \neq 0$;

(ii) se $K(q)<0$, então $\kappa_{1}$ e $\kappa_{2}$ têm sinais opostos. Assim $w$ é uma direção assintótica se $\theta=\arctan \left( \pm \sqrt{-\kappa_{1} / \kappa_{2}}\right)$

(iii) se $K(q)=0$ e $\mathrm{H}(q) \neq 0$, então uma das curvaturas principais é nula e a outra não. Suponha que $\kappa_{1}=0$ e $\kappa_{2} \neq 0$. Logo,

$$
\kappa_{n}(w)=\kappa_{2} \operatorname{sen}^{2} \theta=0
$$

se, e somente se, $w= \pm e_{1}$, isto é, existe uma única direção assintótica.

(iv) se $K(q)=\mathrm{H}(q)=0$, então as curvaturas principais em $q$ são nulas. Assim, para todo $w \in \mathrm{T}_{X(q)} S$ segue que $\kappa_{n}(w)=0$, isto é, toda direção é assintótica.

A partir das direções assintóticas, temos:

Definição A.5.7. Dizemos que uma curva regular $\alpha(t)=X(u(t), v(t)), t \in I$, de uma superfície $X$ é uma curva assintótica se, para todo $t \in I$, o vetor $\alpha^{\prime}(t)$ for uma direção assintótica de $X$ em $(u(t), v(t))$.

Segue direto desta definição o seguinte resultado:

Proposição A.5.8. Uma curva regular $\alpha(t)=X(u(t), v(t))$ é uma curva assintótica se, e somente se, para todo $t \in I$,

$$
e(u(t), v(t)) u^{\prime}(t)^{2}+2 f(u(t), v(t)) u^{\prime}(t) v^{\prime}(t)+g(u(t), v(t)) v^{\prime}(t)^{2}=0 .
$$

Para simplificar a notação escreveremos a equação (A.12) como

$$
e u^{\prime 2}+2 f u^{\prime} v^{\prime}+g v^{\prime 2}=0
$$

chamada equação diferencial das curvas assintóticas da superfície $X$.

Definição A.5.9. Uma parametrização $X: U \subset \mathbb{R}^{2} \rightarrow \mathbb{R}^{3}$ é dita assintótica se as curvas coordenadas são curvas assintóticas.

Proposição A.5.10. Seja $X(u, v),(u, v) \in U \subset \mathbb{R}^{2}$, uma superfície parametrizada regular tal que a função $f$ nunca se anula. Então $X$ é uma parametrização assintótica se, e somente se, $e=0=g$. 
Demonstração. Seja $X$ uma parametrização assintótica e $q=\left(u_{0}, v_{0}\right) \in U$. Como as curvas coordenadas $\alpha(u)=X\left(u, v_{0}\right)$ e $\beta(v)=X\left(u_{0}, v\right)$ são curvas assintóticas então, de (A.12), temos que $e\left(u, v_{0}\right)=0$ e $g\left(u_{0}, v\right)=0$, respectivamente. Em particular, $e\left(u_{0}, v_{0}\right)=g\left(u_{0}, v_{0}\right)=0$.

Por outro lado, se $e=g=0$, a equação (A.13) se reduz a

$$
u^{\prime} v^{\prime}=0
$$

Claramente $u(t)=u_{0} \in \mathbb{R}$ e $v(t)=v_{0} \in \mathbb{R}$ são soluções desta equação diferencial, isto é, as curvas coordenadas são curvas assintóticas.

A existência de curvas assintóticas numa vizinhança de um ponto hiperbólico é garantida pelo resultado que segue.

Proposição A.5.11. Seja $X: U \subset \mathbb{R}^{2} \rightarrow \mathbb{R}^{3}$ uma superfície parametrizada regular. Se $q_{0} \in U$ for um ponto hiperbólico de $X$, então existe uma vizinhança de $q_{0}, V \subset U$, de pontos hiperbólicos tal que, para todo $q \in V$, existem duas curvas assintóticas, $\alpha(t)=X(u(t), v(t))$, satisfazendo $q=(u(0), v(0))$.

Demonstração. Para a prova veja [13], Capítulo 3.

Também podemos parametrizar uma vizinhança de pontos hiperbólicos de uma superfície de tal maneira que as curvas coordenadas são curvas assintóticas, isto é,

Proposição A.5.12. Dados $X: U \subset \mathbb{R}^{2} \rightarrow \mathbb{R}^{3}$ uma superfície parametrizada regular e $q \in U$ um ponto hiperbólico, existe uma vizinhança $U_{0}$ de $q$ e $h: \bar{U}_{0} \subset \mathbb{R}^{2} \rightarrow U_{0}$ uma mudança de parâmetros tal que $X \circ h: \bar{U}_{0} \rightarrow \mathbb{R}^{3}$ é uma parametrização assintótica.

Demonstração. Para a prova veja [13], Capítulo 3.

Relacionado ao conceito de direções assintóticas temos o de direções conjugadas, como segue:

Definição A.5.13. Sejam $X: U \subset \mathbb{R}^{2} \rightarrow \mathbb{R}^{3}$ uma superfície parametrizada regular de traço $S=X(U)$ e $q \in U$. Dizemos que os vetores não nulos $w_{1}, w_{2} \in \mathrm{T}_{X(q)} S$ são conjugados se

$$
\mathrm{II}_{q}\left(w_{1}, w_{2}\right)=0
$$

Observe que se $w_{1}$ e $w_{2}$ são vetores conjugados então, para quaisquer $\lambda_{1}, \lambda_{2}$ reais, os vetores $\lambda_{1} w_{1}$ e $\lambda_{2} w_{2}$ também são conjugados e, portanto, podemos falar em direções conjugadas. É imediato verificar que:

- uma direção assintótica é conjugada a si mesma;

- as direções principais são conjugadas;

- em um ponto umbílico não planar, quaisquer direções ortogonais são conjugadas (pois qualquer direção neste ponto é principal);

- em um ponto planar, quaisquer duas direções são conjugadas. 
Vamos supor que $q \in U$ é um ponto não umbílico com vetores principais $e_{1}, e_{2}$ e com $\kappa_{n}\left(e_{i}\right)=\kappa_{i}, i=1,2$, e considere os vetores unitários

$$
w_{1}=\cos \theta e_{1}+\operatorname{sen} \theta e_{2}, \quad w_{2}=\cos \theta^{\prime} e_{1}+\operatorname{sen} \theta^{\prime} e_{2} .
$$

Note que $w_{1}$ e $w_{2}$ são direções conjugadas se, e somente se,

$$
\kappa_{1} \cos \theta \cos \theta^{\prime}+\kappa_{2} \operatorname{sen} \theta \operatorname{sen} \theta^{\prime}=0
$$

e, neste caso,

$$
\tan \left(\theta^{\prime}-\theta\right)=\frac{\kappa_{1} \cot \theta+\kappa_{2} \tan \theta}{\kappa_{1}-\kappa_{2}}
$$

Observe que a expressão $\kappa_{1} \cot \theta+\kappa_{2} \tan \theta$ se anula, para algum $\theta$ se, e somente se, o ponto $q$ for hiperbólico. Neste caso, da fórmula de Euler segue que $\theta$ e $-\theta$ determinam as direções assintóticas em $q$.

Agora, se a curvatura gaussiana neste ponto for positiva (isto é, $q$ for elíptico), temos que $\theta=\arctan \left( \pm \sqrt{\kappa_{1} / \kappa_{2}}\right)$ e $\theta^{\prime}=\arctan \left(\mp \sqrt{\kappa_{1} / \kappa_{2}}\right)$ determinam as direções conjugadas que formam o menor ângulo. Logo, em um ponto não umbílico de curvatura gaussiana positiva, existe um único par de direções conjugadas que minimiza o ângulo formado por tais direções e que é também simétrico em relação às direções principais. Estas direções são chamadas de direções características da superfície no ponto. 


\section{Apêndice B}

\section{Formas diferenciais}

Se $f$ é uma função diferenciável em $\mathbb{R}^{n}$ a valores reais então a diferencial de $f$ é dada por:

$$
d f=\sum_{i=1}^{n} \frac{\partial f}{\partial x_{i}} d x_{i}
$$

Nem sempre está claro o que esta expressão formal significa. Veremos, assim, o tratamento destas funções usando a noção de formas diferenciais.

Sejam $q$ um ponto de $\mathbb{R}^{n}$ e $\left\{e_{i}\right\}_{i=1, \ldots, n}$ a base canônica de $\mathbb{R}^{n}$. O conjunto de vetores aplicados em $q$, chamado espaço tangente de $\mathbb{R}^{n}$ em $q$, será denotado por $\mathbb{R}_{q}^{n}$. Considere seu espaço dual:

$$
\left(\mathbb{R}_{q}^{n}\right)^{*}=\left\{f: \mathbb{R}_{q}^{n} \rightarrow \mathbb{R} \quad \text { lineares }\right\}
$$

Indicando por $x_{i}: \mathbb{R}^{n} \rightarrow \mathbb{R}$ a função projeção na $i$-ésima coordenada, uma base para $\left(\mathbb{R}_{q}^{n}\right)^{*}$ é obtida tomando $\left(d x_{i}\right)_{q}: \mathbb{R}_{q}^{n} \longrightarrow \mathbb{R}, i=1, \ldots, n$, pois $\left(d x_{i}\right)_{q} \in\left(\mathbb{R}_{q}^{n}\right)^{*} \mathrm{e}$

$$
\left(d x_{i}\right)_{q}\left(e_{j}(q)\right)=\frac{\partial x_{i}}{\partial x_{j}}=\delta_{i j}, \quad i, j=1, \ldots, n,
$$

ou seja, $\left\{\left(d x_{i}\right)_{q}\right\}_{i=1, \ldots, n}$ é a base dual de $\left\{e_{i}(q)\right\}_{i=1, \ldots, n}$.

\section{B.1 Formas diferenciais em $\mathbb{R}^{n}$}

Definição B.1.1. Uma forma de grau 1 (ou uma 1-forma) $\omega$ em um aberto $U \subset \mathbb{R}^{n}$ é uma aplicação que, para cada $q \in U$, associa $\omega_{q} \in\left(\mathbb{R}_{q}^{n}\right)^{*}$, que pode ser escrita na forma:

$$
\omega_{q}=\sum_{i=1}^{n} P_{i}(q)\left(d x_{i}\right)_{q},
$$

$\mathrm{ou}$

$$
\omega=\sum_{i=1}^{n} P_{i} d x_{i}
$$

onde $P_{i}$ são funções de $U$ em $\mathbb{R}$. Se, para cada $i$, a função $P_{i}$ for diferenciável, dizemos que $\omega$ é uma 1-forma diferencial. 
Note que um exemplo de 1-forma diferencial em $U$ é obtido considerando a diferencial da aplicação projeção $x_{i}: U \subset \mathbb{R}^{n} \rightarrow \mathbb{R}, i=1, \ldots, n$.

Definimos a soma de duas 1-formas diferenciais $\omega_{1}$ e $\omega_{2}$ em $U$ por:

$$
\left(\omega_{1}+\omega_{2}\right)_{q}=\left(\omega_{1}\right)_{q}+\left(\omega_{2}\right)_{q}, \quad q \in U,
$$

e, o produto $f w$, onde $f: U \rightarrow \mathbb{R}$ é uma função diferenciável, como a 1-forma diferencial tal que:

$$
(f \omega)_{q}=f(q) \omega_{q}, \quad q \in U .
$$

Com estas operações, o conjunto das 1-formas diferenciais em $U \subset \mathbb{R}^{n}$, denotado por $\Lambda^{1}\left(\mathbb{R}^{n}\right)^{*}$, é um espaço vetorial.

Outra operação entre 1-formas diferenciais é o produto tensorial, definido como segue:

Definição B.1.2. Considere $\omega_{1}$ e $\omega_{2}$ 1-formas diferenciais em $U \subset \mathbb{R}^{n}$. O produto tensorial de $\omega_{1}$ e $\omega_{2}$, denotado por $\omega_{1} \omega_{2}$, é uma aplicação que, para cada $q \in U$, associa uma transformação bilinear:

$$
\begin{aligned}
\left(\omega_{1} \omega_{2}\right)_{q}: \mathbb{R}_{q}^{n} \times \mathbb{R}_{q}^{n} & \longrightarrow \mathbb{R} \\
\left(v_{1}, v_{2}\right) & \longmapsto\left(\omega_{1}\right)_{q}\left(v_{1}\right)\left(\omega_{2}\right)_{q}\left(v_{2}\right) .
\end{aligned}
$$

Note que $\omega_{1} \omega_{2} \neq \omega_{2} \omega_{1}$ e que usaremos a notação $\omega \omega=\omega^{2}$. Temos as seguintes propriedades:

Proposição B.1.3. Sejam $\omega_{1}, \omega_{2}$ e $\omega_{3}$ 1-formas diferenciais em $U \subset \mathbb{R}^{n}$ e $f: U \rightarrow \mathbb{R}$ uma função diferenciável. Então,

(i) $\left(\omega_{1}+\omega_{2}\right) \omega_{3}=\omega_{1} \omega_{3}+\omega_{2} \omega_{3}$;

(ii) $\omega_{1}\left(\omega_{2}+\omega_{3}\right)=\omega_{1} \omega_{2}+\omega_{1} \omega_{3}$;

(iii) $\left(f \omega_{1}\right) \omega_{2}=\omega_{1}\left(f \omega_{2}\right)=f\left(\omega_{1} \omega_{2}\right)$;

(iv) se $\omega_{1}=\sum_{i=1}^{n} P_{i} d x_{i}$ e $\omega_{2}=\sum_{j=1}^{n} Q_{j} d x_{j}$, então

$$
\omega_{1} \omega_{2}=\sum_{i, j=1}^{n} P_{i} Q_{j} d x_{i} d x_{j} .
$$

Aqui temos indicado por $f\left(\omega_{1} \omega_{2}\right)$ a aplicação que, para cada $q \in U$, associa

$$
f(q)\left(\omega_{1} \omega_{2}\right)_{q}: \mathbb{R}_{q}^{n} \times \mathbb{R}_{q}^{n} \longrightarrow \mathbb{R}
$$

Demonstração. Daremos somente a demonstração da última propriedade. As demais seguem direto da definição.

Se $\omega_{1}=\sum_{i=1}^{n} P_{i} d x_{i}$ e $\omega_{2}=\sum_{j=1}^{n} Q_{j} d x_{j}$, então, para cada $q \in U$ e $\left(v_{1}, v_{2}\right) \in \mathbb{R}_{q}^{n} \times \mathbb{R}_{q}^{n}$, temos

$$
\begin{aligned}
\left(\omega_{1} \omega_{2}\right)_{q}\left(v_{1}, v_{2}\right) & =\left(\omega_{1}\right)_{q}\left(v_{1}\right)\left(\omega_{2}\right)_{q}\left(v_{2}\right)=\left(\sum_{i=1}^{n} P_{i}(q)\left(d x_{i}\right)_{q}\left(v_{1}\right)\right)\left(\sum_{j=1}^{n} Q_{j}(q)\left(d x_{j}\right)_{q}\left(v_{2}\right)\right) \\
& =\sum_{i, j=1}^{n} P_{i}(q) Q_{j}(q)\left(d x_{i}\right)_{q}\left(v_{1}\right)\left(d x_{j}\right)_{q}\left(v_{2}\right) \\
& =\sum_{i, j=1}^{n}\left(P_{i} Q_{j}\right)(q)\left(d x_{i} d x_{j}\right)_{q}\left(v_{1}, v_{2}\right)=\left(\sum_{i, j=1}^{n} P_{i} Q_{j} d x_{i} d x_{j}\right)_{q}\left(v_{1}, v_{2}\right)
\end{aligned}
$$

e, portanto, segue o resultado. 
Seja, agora, $\Lambda^{2}\left(\mathbb{R}_{q}^{n}\right)^{*}$ o conjunto das aplicações

$$
\phi: \mathbb{R}_{q}^{n} \times \mathbb{R}_{q}^{n} \longrightarrow \mathbb{R}
$$

bilineares (isto é, linear em cada variável) e alternadas (ou seja, $\phi\left(v_{1}, v_{2}\right)=-\phi\left(v_{2}, v_{1}\right)$ ). Definindo em $\Lambda^{2}\left(\mathbb{R}_{q}^{n}\right)^{*}$ as operações usuais de funções, este torna-se um espaço vetorial.

Definição B.1.4. Dadas $\omega_{1}$ e $\omega_{2}$ 1-formas diferenciais em $U \subset \mathbb{R}^{n}$, definimos o produto exterior de $\omega_{1}$ e $\omega_{2}$, denotado por $\omega_{1} \wedge \omega_{2}$, como sendo a aplicação que, para cada $q \in U$, associa $\left(\omega_{1} \wedge \omega_{2}\right)_{q} \in \Lambda^{2}\left(\mathbb{R}_{q}^{n}\right)^{*}$ definido por:

$$
\left(\omega_{1} \wedge \omega_{2}\right)_{q}\left(v_{1}, v_{2}\right)=\operatorname{det}\left(\left(\omega_{i}\right)_{q}\left(v_{j}\right)\right)=\left(\omega_{1} \omega_{2}\right)_{q}\left(v_{1}, v_{2}\right)-\left(\omega_{2} \omega_{1}\right)_{q}\left(v_{1}, v_{2}\right), \quad\left(v_{1}, v_{2}\right) \in \mathbb{R}_{q}^{n} \times \mathbb{R}_{q}^{n} .
$$

Note que $\left(d x_{i} \wedge d x_{j}\right)_{q} \in \Lambda^{2}\left(\mathbb{R}_{q}^{n}\right)^{*}$ e que $\left\{\left(d x_{i} \wedge d x_{j}\right)_{q}, i<j\right\}$ forma uma base para o espaço $\Lambda^{2}\left(\mathbb{R}_{q}^{n}\right)^{*}$ (veja [11], página 4). Além disso, decorre desta definição que, para todo $q \in U$ e $i, j=1, \ldots, n$,

$$
\begin{aligned}
& \text { - }\left(d x_{i} \wedge d x_{j}\right)_{q}\left(\frac{\partial}{\partial x_{i}}, \frac{\partial}{\partial x_{j}}\right)=1 ; \\
& \text { - } d x_{i} \wedge d x_{j}=-d x_{j} \wedge d x_{i} ; \\
& \text { - } d x_{i} \wedge d x_{i}=0
\end{aligned}
$$

Assim, temos as seguintes propriedades:

Proposição B.1.5. Considerando as 1-formas diferenciais $\omega_{1}, \omega_{2}$ e $\omega_{3}$, resulta

1. $\omega_{1} \wedge\left(\omega_{2}+\omega_{3}\right)=\omega_{1} \wedge \omega_{2}+\omega_{1} \wedge \omega_{3}$

2. Se $\omega_{1}=\sum_{i=1}^{n} P_{i} d x_{i}$ e $\omega_{2}=\sum_{j=1}^{n} Q_{j} d x_{j}$, então

$$
\omega_{1} \wedge \omega_{2}=\sum_{i=1}^{n-1} \sum_{j=i+1}^{n} P_{i} Q_{j} d x_{i} \wedge d x_{j}-\sum_{i=2}^{n} \sum_{j=1}^{i-1} P_{i} Q_{j} d x_{j} \wedge d x_{i} ;
$$

3. $\omega_{1} \wedge \omega_{2}=-\omega_{2} \wedge \omega_{1}$;

4. $\omega_{1}$ e $\omega_{2}$ são linearmente independentes se, e somente se, $\omega_{1} \wedge \omega_{2} \neq 0$, isto é, $\left(\omega_{1} \wedge \omega_{2}\right)_{q} \neq 0$, para todo $q \in U$.

Indicamos, agora, por $\Lambda^{k}\left(\mathbb{R}_{q}^{n}\right)^{*}$ o conjunto das aplicações $k$-lineares e alternadas:

$$
\phi: \underbrace{\mathbb{R}_{q}^{n} \times \ldots \times \mathbb{R}_{q}^{n}}_{k \text { vezes }} \longrightarrow \mathbb{R}
$$

Com as operações usuais, $\Lambda^{k}\left(\mathbb{R}_{q}^{n}\right)^{*}$ é um espaço vetorial. Se considerarmos $\omega_{1}, \ldots, \omega_{k} 1$-formas diferenciais em $U \subset \mathbb{R}^{n}, \omega_{1} \wedge \ldots \wedge \omega_{k}$ é a aplicação que, para cada $q \in U$, associa $\left(\omega_{1} \wedge \ldots \wedge \omega_{k}\right)_{q} \in$ $\Lambda^{k}\left(\mathbb{R}_{q}^{n}\right)^{*}$ definida por:

$\left(\omega_{1} \wedge \ldots \wedge \omega_{k}\right)_{q}\left(v_{1}, \ldots, v_{k}\right)=\operatorname{det}\left(\left(\omega_{i}\right)_{q}\left(v_{j}\right)\right), \quad\left(v_{1}, \ldots, v_{k}\right) \in \mathbb{R}_{q}^{n} \times \ldots \times \mathbb{R}_{q}^{n}, \quad i, j=1, \ldots, k$.

O conjunto $\left\{\left(d x_{i_{1}} \wedge \ldots \wedge d x_{i_{k}}\right)_{q}, i_{1}<\ldots<i_{k}, i_{j} \in\{1, \ldots, n\}\right\}$ forma uma base para o espaço $\Lambda^{k}\left(\mathbb{R}_{q}^{n}\right)^{*}$.

Podemos, então, dar a seguinte: 
Definição B.1.6. Uma $k$-forma diferencial em $U \subset \mathbb{R}^{n}, k \geq 1$, é uma aplicação $\phi$ que para cada $q \in U$ associa $\phi_{q} \in \Lambda^{k}\left(\mathbb{R}_{q}^{n}\right)^{*}$ que pode ser escrita da forma:

$$
\phi_{q}=\sum_{i_{1}<\ldots<i_{k}} P_{i_{1} \ldots i_{k}}(q)\left(d x_{i_{1}} \wedge \ldots \wedge d x_{i_{k}}\right)_{q},
$$

onde a soma é sobre todas as k-uplas $\left(i_{1}, \ldots, i_{k}\right), i_{1}<\ldots<i_{k}, i_{j}=1, \ldots, n$, e $P_{i_{1} \ldots i_{k}}: U \rightarrow \mathbb{R}$ são funções diferenciáveis.

Usa-se, por convenção, que uma função diferenciável $f: \mathbb{R}^{n} \rightarrow \mathbb{R}$ é uma 0-forma diferencial em $\mathbb{R}^{n}$. Neste caso observe que $d f$ é uma 1-forma diferencial. Vamos, assim, introduzir a noção de diferencial exterior de uma 1-forma diferencial, obtendo uma 2-forma diferencial.

Definição B.1.7. Dada uma 1-forma $\omega=\sum P_{i} d x_{i}$, definimos a diferencial exterior de $\omega$ como sendo a 2-forma dada por:

$$
d \omega=\sum_{i=1}^{n} d P_{i} \wedge d x_{i} .
$$

Proposição B.1.8. Considerando $\omega_{1}, \omega_{2}$ 1-formas diferenciais, são válidas as seguintes propriedades:

1. $d\left(\omega_{1}+\omega_{2}\right)=d \omega_{1}+d \omega_{2} ;$

2. $d\left(\omega_{1} \wedge \omega_{2}\right)=d \omega_{1} \wedge \omega_{2}-\omega_{1} \wedge d \omega_{2}$;

3. $d\left(d \omega_{1}\right)=0$.

Demonstração. A prova é encontrada em [11], página 17.

Definição B.1.9. Dizemos que uma 1-forma diferencial $\omega$ é exata se existe uma função $\theta: U \rightarrow$ $\mathbb{R}$ diferenciável tal que $\omega=d \theta$. Dizemos que $\omega$ é fechada se $d \omega=0$.

\section{B.2 Teorema de Frobenius}

Usando a teoria de formas diferenciais, vamos apresentar nesta seção uma versão do Teorema de Frobenius que foi muito utilizada durante nosso estudo.

Denote por $J$ o ideal gerado pelas 1-formas linearmente independentes $\omega_{1}, \ldots, \omega_{k}$, isto é, o conjunto de todas as formas do tipo $\phi=\sum_{i=1}^{k} \alpha_{i} \wedge \omega_{i}$, onde $\alpha_{i}$ são formas diferenciais em $\mathbb{R}^{n}$. Dizemos que $J$ é um ideal diferencial fechado se $d \phi \in J$ sempre que $\phi \in J$. Portanto, podemos verificar o seguinte resultado:

Proposição B.2.1. O ideal gerado pelas 1-formas $\omega_{1}, \ldots, \omega_{k}$ é um ideal fechado se, e só se, existem 1-formas $\omega_{i j}$ tal que

$$
d \omega_{j}=\sum_{i=1}^{k} \omega_{i} \wedge \omega_{i j}, \quad j=1, \ldots, k .
$$

Agora, podemos enunciar o Teorema de Frobenius como segue:

Teorema B.2.2 (Teorema de Frobenius). O sistema diferencial $\omega_{1}=\ldots=\omega_{k}=0$ é integrável se, e só se, o ideal $J$ gerado por $\omega_{1}, \ldots, \omega_{k}$ é um ideal diferencial fechado, isto é, $d J \subset J$.

Demonstração. A prova deste resultado pode ser encontrada no Apêndice de [23]. 


\section{Bibliografia}

[1] S.C. de Almeida, F.A.A. Pimentel, O Teorema de Bäcklund segundo S. S. Chern. Matemática Universitária, no 20/21 (1996), 23-45.

[2] P.V. Araújo, Geometria Diferencial. Coleção Matemática Universitária, IMPA, 2004.

[3] A.V. Bäcklund, Einiges über Curve und Flächentransformationen. Lund Universiët Arsskrift, 10 (1875).

[4] A.V. Bäcklund, Concerning surfaces with constant negative curvature, translated by E. M. Coddington, New Era Printing Co., Lancaster pa, 1905.

[5] L. Bianchi, Sulla teoria delle trasformazioni delle superficie d'area minima. Rend. Acc. Naz. Lincei 58 (1899), 151-165.

[6] L. Bianchi, Sulla teoria delle trasformazioni delle superficie a curvatura costante. Ann. Mat. Pura Appl. 33 (1899), 185-298.

[7] L. Bianchi, Sulla transformazioni di Bäcklund per le superficie pseudosferiche. Rend. Acc. Naz. Lincei 51 (1892), 3-12.

[8] R. Caddeo, A. Gray, Curve e Superfici, traduzione italiana ampliata del libro Modern Differential Geometry of Curves and Surfaces with MATHEMATICA, di A. Gray, CRC Press, (1998).

[9] M.A. Camacho, Aplicación del teorema fundamental de superficies en $\mathbb{R}^{3}$ en la ecuación de Sine-Gordon. Mosaicos Matemáticos, Sorona, México, 20 (2007), 2329.

[10] M.P. do Carmo, Differential Geometry of Curves and Surfaces. Prentice - Hall, 1976.

[11] M.P. do Carmo, Formas diferenciais e aplicações. Poços de Caldas, 1971.

[12] M.P. do Carmo, O método do referencial móvel. III Esc. Lat. Amer. de Mat., IMPA, 1976.

[13] M.P. do Carmo, Geometria Diferencial de Curvas e Superfícies. SBM, 2005.

[14] É. Cartan, Les Systèmes Différentiel Extérieurs et Leurs Applications Géométriques. Herman, Paris, 1946.

[15] S.S. Chern; C. L. Terng, An analogue of Bäcklund's theorem in affine geometry. Rocky Mountain J. Math., 10 (1980), 105-124. 
[16] C. Gu; H. Hu; Z. Zhou, Darboux transformations in integrable systems. Springer, 26 (2005), 121-187.

[17] C. Guichard, Sur la déformation des quadriques de révolution. Comptes Rendus de l'Académie des Sciences (1899), 232-233.

[18] T.A. Ivey; J.M. Landsberg, Cartan for Beginners: Differential Geometry via Moving Frames and Exterior Differential Systems. American Mathematical Society Providence, 1963.

[19] B. O'Neill, Elementary Differential Geometry. Academic Press, 1966.

[20] C. Rogers, W.K. Schief, Bäcklund and Darboux Transformations: Geometry and Modern Applications in Soliton Theory. Cambridge University Press, 2003.

[21] M. Spivak, A Comprehensive Introduction to Differential Geometry. Publish or Perish, 1979.

[22] K. Tenenblat, Introdução à Geometria Diferencial. Editora UnB, 1988.

[23] K. Tenenblat, Transformações de Superfícies e Aplicações. XIII Col. Bras. de Mat., IMPA, 1981.

[24] K. Tenenblat, Transformations of Manifolds and Applications to Differential Equations. Addison Wesley Longman, Pitman Monographs and Surveys in Pure and Applied Mathematics, 1998.

[25] C.L. Terng, Lecture Notes on Curves and Surfaces in $\mathbb{R}^{3}$. Preliminary version and in progress, 2003. 